\title{
Sur le problème d'équivalence de certaines structures infinitésimales.
}

\author{
Múmoire de Paulette Libermann (à Strasbourg).
}

Résnme. Après avoir rappelé la défnition et quelques propriétés des pseudogroupes de Lie, on definit les structures infinitésimales régulières. On peut toujours associer des connexions affines $\dot{a}$ de telles structures; courbure et torsion de ces, connexions. Equivalence locale de deux structures infinitésimales. Application à l'étude des structures presque complexes, presque paracomplexes, presque symplectiques, presque hermitiennes, presque parahermi. tiennes, presque quaternioniennes, presque quaternioniennes de deuxième espèce.

\section{INTRODUCTION}

La théorie de l'équivalence développée par E. CARTaN dans de nombreux mómoires (notamment dans [10] et [13]) .(*) est à la base de la géométrie différentielle; elle permet également l'intégration de certains systèmes diffé. rentiels.

L'exposé de cette théorie fait l'objet de la première partie du présent travail; le problème d'équivalence est formulé en le rattachant à la théorie des espaces fibrés, en particulier en introduisant la notion de structure infinitésimale régulière dûe à C. EHRESMANN [26]: une structure infinitésimale régulière da premier ordre sur une variété différentiable $V_{n}$ est une structure fibrée subordonnée à la structure fibrée de l'espace des vecteurs tangents à $V_{n}$ (le groupe structural $G$ est un sous-groupe du groupe linéaire homogéne $L_{n}$, la fibre est $R^{n}$. Le problème d'équivalence de E. CARTAN se ramène au problème d'équivalence locale de ces structures; nous n'aborderons pas le problème d'existence de telles structures qui conduit à des «obstacles», ni celui de l'équivalence globale.

Dans la recherche des conditions d'équivalence locale de deux structures on est amené à étudier le pseudogroupe de leurs antomorphismes locaux, d'où l'introduction de la notion de pseudogroupe fini ou infini de LIE ("groupe fini » ou «groupe infini » de LIE suivant la terminologie de $E$. Cartan). C'est d'ailleurs l'étude du problème d'equivalence qui a conduit E. CarTan à édifier sa théorie des «groupes infiniss» de LIE, que l'on trouve exposée notamment dans [9] et [12] ; les transformations de ce "groupe infini 》 constituent l'intégrale générale d'un système d'équations aux dérivées partielles que l'on peut ramener à un système de PFaff en involution.

Dans le chapitre I, consacré aux pseudogroupes de LIE, je rappelle d'abord

(*) Les nombres entre crochets renvoient à l'index bibliographique. 
quelques propriétés des systèmes d'équations aux dérivées partielles et les principales notions de la théorie des systèmes de PFAFF en involution. Ensuite est exposée la définition d'un pseudogroupe de LIE, telle qu'elle a été donnée par C. EhresmanN [26] en utilisant la notion de groupoïde de jets; un pseudogroupe de LIE est caractérisé par son ordre; de plus j' introduis, pour les pseudogroupes finis, la notion de degré. Les deux thécèmes fondamentaux de E. CARTAN relatifs à la théorie dés psendogroupes de LIE sont démontrés en utilisant la notion de prolongement d' une variété différentiable [26], notion déjà utilisée bien que non formulée explicitement par U. AMaLdi dans son ourrage sur les "groupes infinis» [1]. En vertu du premier théorème de E. CarTaN, tout pseudogroupe de Lite admet un prolongement du premier ordre et, s'il est fini, un prolongement d'ordre et de degré égaux à 1. Dans le deuxième théorème sont énoncées des conditions pour qu' un pseudogroupe de transformations soit un psendogroupe infini (resp. fini) de LIE d'ordre 1 (resp. d'ordre et de degré égaux à 1).

Dans le chapitre II est définie l'équivalence locale de deux structures infinitésimales régulières. Une structure infinitésimale régulière est déterminée localement par un ensemble de $n$ formes de PFafF dont la restriction $\grave{a}$ tout point de $V_{n}$ est un «corepère ». On envisage en particulier des structures isotropes, localement homogènes, intégrables. A toute structure infinitésimale régulière sont associées des connexions affines [25]; à chacune des connexions correspond un tenseur de courbure et un tenseur de torsion. Je démontre deux thérèmes relatifs aux structures infinitésimales régulières integrables. J'expose ensuite les méthodes de E. CARTAN pour traiter le problème d'équivalence restreint (structures dont le groupe structural est la transformation identique). Dans la résolution da problème d'équivalence général, on oherche à déterminer en chaque point un corepère distingué; si cette détermination est possible, on est ramené au problème d'équivalence restreint. Lorsqu' on peut associer canoniquement à la structure une connexion affine, le pseudogroupe de ses antomorphismes est un pseudogroupe de LIE de type fini de degré 2 ; dans le cas contraire, le pseudogroupe de ses automorphismes locaux est en général un psendogroupe infini de LrE (si l'on suppose de plus les données analytiques).

Les théories générales exposées dans la première partie de ce travail ont leurs applications dans la deuxième partie, consacrée aux structures infinitésimales régulières définies sur une variété $V_{2 n}$, de dimension $2 n$, dont le groupe structural $G$ est: le groupe linéaire homogène complexe $L_{n}^{\prime}$ on le groupe linéaire homogène "paracomplexe» $L_{n}^{\prime}$ (isomorphe a $L_{n} \times L_{n}$ ) ou encore le groupe symplectique $\tilde{L}_{2 n}$; ces structures sont appelées respectivement presque complexes, presque "paracomplexes», presque symplectiques. On étudiera également dans cette deuxième partie des structures subordonnées aux précédentes, dont le groupe structural est isomorphe a une forme réelle 
du groupe $L_{n}^{\prime}$ : groupe unitaire $U_{n}$, groupe $U_{n}^{(k)}$ unitaire « d'espèce $k$ », groupe «para-unitaire» $\dot{U}_{n}$ et groupe $\widehat{L}_{n}$ (ces deux derniers isomorphes à $L_{n}$ ) et si $n=2 p$, groupe linéaire homogène quaternionien $L_{p}^{\prime \prime}$ et quaternionien «de deuxième espèce $\stackrel{L}{L}_{p}{ }_{p}$ (identique à $\widehat{L}_{2 p}$ ). Contrairement à ce qui a lieu pour les structures presque complexes, presque paracomplexes et presque symplectiques, on peut associer canoniquement au moins une connexion affine à ces structures (structures presque hermitiennes, presque hermitiennes "d'espèce $\mathrm{k} \gg$, presque parahermitiennes, presque quaternioniennes, presque quaternioniennes de deuxième espèce). Pour chacune de ces connexions, on peut définir, outre les tenseurs de courbure et de torsion, des tenseurs de courbure et de torsion conformes. La recherche de celles de ces structures qui sont isotropes conduit aux structures intégrables et aux structures localement équivalentes à une structure d'espace hermitien elliptique ou hyperbolique (si le groupe structural est $U_{n}$ ), d'espace hermitien d'espèce $k$ (si le groupe structural est $U_{n}^{(k)}$ ) ou a une structure parahermitienne sur la variété $P_{n}(R) \times P_{n}(R)$ (si le groupe structural est $\dot{U}_{n}$ ). On obtient ainsi des espaces riemanniens symétriques à métrique définie ou indéfinie. En définissant une isotropie restreinte, on est conduit aux structures précédentes et en outre à des structures localement équivalentes à une structure presque hermitienne sur la sphère $S_{6}$ ou à une structure presque parahermitienne sùr la quadrique réelle $Q_{6}$; ces structures admettent respectivement come groupe d'automorphismes le groupe simple compact $G_{2}$ a 14 parametres et le groupe simple $G_{2}^{\prime}$ qui sont les deux formes réelles d' un même groupe complexe.

Dans le chapitre III (qui est purement algébrique) sont étudiées des structures de la fibre $R^{2 n}$ admettant comme groupe d'automorphismes $L_{n}^{\prime}$, $\stackrel{\circ}{L}_{n}, \tilde{L}_{2 n}, U_{n}, U_{n}^{(k)}, \stackrel{\circ}{U}_{n}, \widehat{L}_{n}$. Dans le dernier paragraphe de ce chapitre est définie l'adjointe d' une forme exterieure $\varphi$ par rapport à une forme extérieure quadratique $\Omega$ de rang $2 n$; l'opérateur $\Lambda$, adjoint de l'opérateur $L \varphi=\varphi \wedge \mathbf{\Omega}$ ne dépend que de $\Omega$ et non de la forme quadratique définie positive échan. geable avec $\Omega$; il en est de même de la notion de classe; il est egalement prouvé que la décomposition d'une forme en formes de classe déterminée (dont je donne une démonstration différente de celle d'Eokmann-GuggenHEIMER) est identique à la décomposition de LEPAGE.

Ces résultats sont utilisés dans le chapitre IV, relatif au problème d'équivalence des formes différentielles extérieures quadratiques, et dans lequel est introduite la notion de codifférentielle par rapport à une forme différentielle extérieure quadratique.

Dans le chapitre $V$ sont étudiées les structures presque complexes et presque paracomplexes, ainsi que les structures presque hermitiennes, presque hermitiennes d'espèce $k$, presque parahermitiennes. En particulier, le problème d'equivalence des structures presque complexes et presque hermitiennes sur une variété $V_{4}$ est traité en détail. 
Enfin les structures infinitésimales régulières de groupe structural $\widehat{L_{n}}$ (structures presque quaternioniennes de deuxième espèce si $n=2 p$ ) font l'objet du chapitre VI; à de telles structures on peut associer canoniquement trois connexions affines en général distinctes. On envisage également dans ce chapitre des structures subordonnées aux précédentes, de groupe structural isomorphe an groupe orthogonal $O_{n}$ (on determine celles de ces structures qui sont isotropes); si $n=2 p$, on envisage aussi des structures de groupe structural isomorphe à $L_{p} \times L_{p} \times L_{p} \times L_{p}$ ou à $L_{p}{ }^{\prime} \times L_{p}{ }^{\prime}$ ou à un de leurs sous-groupes; parmi ces dernières, celles dont le groupe structural est isomorphe a $L_{p}^{\prime}$ et qui sont isotropes sont ou bien intégrables ou bien localement équivalentes à une structure parahermitienne complexe sur la variété $P_{p}(C) \times P_{p}(C)$.

Qu'il me soit permis d'exprimer ma profonde reconnaissance à Monsieur Charles Ehresmann pour l'aide qu'il a bien voulu $\mathbf{m}^{\prime}$ accorder dans mes recherches et dont les nombreux conseils ont été précieux dans l'élaboration de ce travail.

\section{PREMIERE PARTIE}

Chapitris I.

\section{Pseudogroupes finis et infinis de Lie.}

\section{1. - Systèmes d'équations anx dérivées partielles complètement intégrables} et systèmes de Pfaff en involution ( $\left.{ }^{1}\right)$.

A) Nous rappellerons d'abort la notion de jet [26]: soit $f$ une application d'un voisinage $U$ d'un point $x_{0}$ d'une variété différentiable $V_{n}$ (de dimension. $n$ ) dans une variété différentiable $V_{p}$ (de dimension $p$ ) s'exprimant à l'aide des coordonnées locales de $x \in U$ et $y=f(x)$ par des fonctions $f^{\prime}$ admettant des dérivées partielles continues d'ordre au moins égal à $q$; lo jet $j_{x}^{q} f$ d'ordre $q$ de la fonction $f$ (jet de source $x$, de but $y$ ) est la classe des applications $g$ d'un voisinage de $x$ dans $V_{p}$, appliquant $x$ sur $y$, telles que les dérivées partielles des $g^{i}$ d'ordre inférieur ou égal à $q$ prennent en $x$ la mème valeur que celles de même espèce des $f^{i}$. L'ensemble de tous les $q$-jets de $V_{n}$ dans $V_{p}$ est une variété $J^{q}\left(V_{n}, V_{p}\right)$. La variété $J^{\circ}\left(V_{n}, V_{p}\right)$ est identifiée à $V_{n} \times V_{p}$. Si $r<q$, tout $q$-jet $X^{q} \varepsilon J^{q}\left(V_{n}, V_{p}\right)$ détermine un jet $X^{r} \varepsilon$ $J^{r}\left(V_{n}, V_{p}\right)$ ou projection de $X^{q}$ dans $J^{r}\left(V_{n}, V_{p}\right)$. Soit $\left(X^{q}\right)_{0} \varepsilon J^{q}\left(V_{n}, V_{p}\right)$ un jet de source $x_{0}$, de but $y_{0}$. Si $x^{i}(i=1, \ldots, n)$ et $y^{\lambda}(\lambda=1, \ldots, p)$ sont respectivement des systèmes des corrdonnees locales sur $V_{n}$ et $V_{p}$ au voisinage de $x_{0}$ et $y_{0}$, l'ensemble des $x^{i}, y^{\lambda}, \frac{\partial^{x_{1}}+\ldots+x_{n} y^{\lambda}}{\left(\partial x^{1}\right)^{\alpha_{1} \ldots}\left(\partial x^{n}\right)^{\alpha_{n}}}\left(\alpha_{1}+\alpha_{2}+\ldots+\alpha_{n}=1, \ldots, q\right)$ cons.

(1) Pour les systèmes de Prayf voir E. Cartan [9], [14] of H. Cartan [16]. Nous utiliserons la terminologie de E. Cartan. 
titue un système de coordonnées locales sur $J q\left(V_{n}, V_{p}\right)$ dans un voisinage $W^{\top}$ de $\left(X^{q}\right)_{0}$. Tout système $\Sigma^{q}$ d'équations aux derivées partielles d'ordre $q$

$$
\Phi_{l}\left(x^{i}, y^{\lambda} \frac{\partial^{\alpha_{1}+\ldots+\alpha_{n}} y^{\lambda}}{\left(\partial x^{1}\right)^{\alpha_{1}} \ldots\left(\partial x^{n}\right)^{\alpha_{n}}}\right)=0 \begin{aligned}
& (l=1, \ldots, m) \\
& (i=1, \ldots, n) \\
& (\lambda=1, \ldots, p) \\
& \left(\alpha_{1}+\alpha_{2}+\ldots+\alpha_{n}=1, \ldots, q\right)
\end{aligned}
$$

définit un sous-ensemble $M^{q}$ de $J^{q}\left(V_{n}, V_{n}\right)$ dont les jets appartiennent à $W$. Nous supposerons désormais que les $\Phi_{l}$ sont des fonctions analytiques des coordonnées locales dans $W$ et le sous-ensemble $M^{q}$ sera appelé variété analytique élémentaire extraite de $J^{q}\left(V_{n}, V_{p}\right)$. Deux systèmes d'equations aux dérivées partielles définissant la même variété $M^{q}$ seront dits équivalents. Soit $f$ une application analytique de $V_{n}$ dans $V_{p}$ définie dans une partie de $U$; désignons par $\Pi^{q}(f)$ le sous-ensemble de $J^{q}\left(V_{n}, V_{p}\right)$ engendré par tous les jets $j_{x}^{q} f$ quand $x$ parcourt $U$. Une solution analytique du système $\Sigma^{q}$ est une application analytique $f$ telle que: $\Pi^{q}(f) \subset M^{q}$. (Nous ne considérerons que des solutions analytiques). Soit $\Pi^{q}$ la réunion de tous les $\Pi^{q}(f)$ correspondant a l'ensemble des solutions $f$ de $\Sigma^{q}$. On a: $\Pi^{q} \subset M^{q}$.

Le système $\Sigma^{q}$ est dit complètement intégrable si $\Pi^{q}=M^{q}$, c'est-à-dire si a tout jet $X^{q} \& M^{q}$ correspond au moins une solution $f$ de $\Sigma^{q}$ telle que $X^{q} \varepsilon \Pi^{q}(f)$. Le prolongement d'ordre $q+k$ d'un système d'équations aux dérivées partielles $\Sigma^{q}$ est le système $\Sigma^{q+k}$ d'ordre $q+k$ obtenu par dérivations totales successives des équations de $\Sigma^{q}$ par rapport à toutes les variables indépendantes. Ce système $\Sigma^{q+k}$ définit une variété elémentaire $M^{q+k}$ extraite de $J^{q+h}\left(V_{n}, V_{p}\right)$ dont tout jet $X^{q+k}$ se projette dans $J^{q}\left(V_{n}, V_{p}\right)$ suivant un jet $X^{q} \in M^{q}$; les jets de $M^{q+k}$ appartiennent à un ouvert $W_{(q+k)}$ de $J^{q+k}\left(V_{n}, V_{p}\right)$ se projetant dans $J^{q}\left(V_{n}, V_{p}\right)$ suivant $W$. Toute solution de $\Sigma^{q}$ est solution de $\Sigma^{q+k}$ et inversement. Si le système $\Sigma^{q}$ est complètement intégrable, à tout jet $X^{q} \varepsilon M^{q}$ correspond au moins un jet $X^{q+k} \varepsilon M^{q+k}$ admettant $X^{q}$ comme projection : en effet si $f$ est une solution de $\Sigma^{q}$ telle $q u e j_{x}^{q} f=X^{q}$, le jet $X_{x}^{q+k} f$ appartient à $M^{q-k}$ et admet $X^{q}$ come projection. Done pour que le système $\Sigma^{q}$ soit complètement intégrable, il faut que, quel que soit $k$, la projection de la variété extraite $M^{q+k}$ dans $J^{q}\left(V_{n}, V_{p}\right)$ soit une application de $M^{q+k}$ sur $M^{q}$ ou encore: il faut que toute équation aux dérivées partielles d'ordre $\leq q$ d'un prolongement quelconque de $\Sigma^{q}$ soit vérifiée identiquement en tenant compte des équations de $\Sigma^{q}$. Le prolongement d'un système complè. tement intégrable $\Sigma^{q}$ n'est pas nécessairement complètement intégrable. Pour que tout prolongement de $\Sigma^{q}$ soit complètement intégrable, il faut que la projection de $M^{q+k+s}$ dans $J^{q+r}\left(V_{n}, V_{p}\right)$ soit une application de $M^{q+k+s}$ sur $M^{q+k}$ (quels que soient $k$ et $s$ ). Un système $\Sigma^{q}$ joujssant de cette propriété est appele passif suivant la terminologie de RIQUIER [43].

Supposons que la variété élémentaire $M^{q}$ puisse être définie par un sys. 
tème $\Sigma^{q}$ ayant la forme suivante: pour $s=0,1, \ldots, q$, toutes les dérivées d'ordre $s$ sout des fonctions analytiques des variables indépendantes et de certaines dérivées (appelées dérivées paramétriques) dont l'ordre est inférieur ou égal à $s$, le jacobien $j_{s}$ des $\frac{\partial^{\alpha_{1}+\ldots+\alpha_{n}} y^{\prime}}{\left(\partial x^{1}\right)^{\alpha_{1}} \ldots\left(\partial x^{n}\right)^{\alpha_{n}}}\left(\alpha_{1}+\ldots+\alpha_{n}=s\right)$ par rapport à l'ensemble des $x^{i}$ et des dérivées paramétriques d'ordre $s$ ayant un rang constant $m_{s}$. Le système $\Sigma^{q}$ définit une représentation paramétrique de la variété extraite $M^{q}$; le jacobien $j_{q}$ ayant un rang constant $m_{q}$ pour tous les jets $X^{q} \varepsilon M^{q}$, ces jets sont des points réguliers de cette variété extraite (dont la dimension est $\left.m_{q}\right)$. La projection $M^{s}$ de $M^{q}$ dans $J^{s}\left(V_{n}, V_{v}\right)$ est définie par le système $\Sigma^{s}$ constitué par l'ensemble des équations d'ordre $\leq s$ figurant dans $\Sigma^{q}: M^{s}$ est une variété élémentaire extraite de $J^{s}\left(V_{n}, V_{p}\right)$ dont tous les points sont régulier's et dont la dimension est $m_{\varepsilon}$. C'est pourquoi nous dirons que $M^{q}$ est complètement régulière et que le système $\Sigma^{q}$ est complètement régulier. La variété extraite $M^{q}$ est alors une sous-rariété de $J^{q}\left(V_{n}, V_{p}\right)$ et sera appelée sous-variété élémentaire complètement régulière.

On peut done énoncer la proposition suivante: les projections dans $J^{\circ}\left(V_{n}, V_{p}\right), \ldots, J^{q-1}\left(V_{n}, V_{p}\right)$ d'une sous-variété élémentaire complètement régulière de $J^{q}\left(V_{n}, V_{p}\right)$ sont des sous-variétés complètement régulières.

Un système complètement régulier $\Sigma^{q}$ est dit de MAYER-LIE s' il existe un nombre $r \leq q$ tel que l'ordre maximum des dérivées paramétriques soit égal à $r-1$; nous désignerons le nombre $r$ par degré du système. Lorsque le système $\Sigma q$ est complètement intégrable, il en est de même du système complètement régulier $\Sigma^{r}$ définissant la sous-variété $M^{r}$. Alors par différentiations successives des équations de $\Sigma^{r}$, on obtient une expression de toute dérivée $\frac{\partial^{x_{1}}+\ldots+x_{n} y^{\prime}}{\left(\partial x^{1}\right)^{\alpha_{1}} \ldots\left(\partial x^{n}\right)^{\alpha_{n}}}$ d'ordre $\alpha+\ldots+\alpha_{n}>r$ en fonction des dérivées d'ordre $\leq r-1$ (si l'on calcule une dérivée de deux manières différentes, les conditions de compatibilité ne font intervenir que des dérivées d'ordre $\leq r-1$ et sont donc identiquement vérifiées). Il en résulte que: $1^{0} \Sigma^{q}$ est équivalent à un prolongement de $\Sigma^{r}$ et par conséquent toute solution de $\Sigma^{q}$ est solution de $\Sigma^{r} ; 2^{\circ}$ les prolongements successifs de $\Sigma^{q}$ sont complètement intégrables et complètement réguliers. Comme à tout jet $X^{r-1} \varepsilon M^{r-1}$ correspond un jet et un seul $X^{r-1+s} \varepsilon M^{r-1+s}$ (quel que soit s), la projection de $M^{r-1+s}$ sur $M^{r-1}$ est un isomorphisme. A tout jet $X^{r-1} \varepsilon M^{r-1}$ corrrespond (dans un voisinage suffisamment petit de la source de $X^{r-1}$ ) une solution de $\Sigma^{r}$ et une seule.

Soit $\Sigma^{q}$ un système complètement intégrable et complètement régulier; qui ne soit pas de MaYER-LIE, mais supposons que pour le prolongement $\Sigma^{q+1}$ les dérivées d'ordre $q+1$ s'expriment en fonction des dérivées d'ordre $\leq q$; le système $\Sigma^{q+1}$ est alors un système de MaYER-Lie complètement intégrable de degré $q$. On démontre comme précédemment que tous les prolongements successifs de $\Sigma^{q+1}$ sont complètement réguliers et complètement intégrables. 
$B$ ) Soit dans le voisinage $U$ d'un point $x_{0}$ d'une variété différentiable $V_{m}$ le système de Pfaff $S$ :

$$
\begin{array}{cc}
f_{l}\left(x^{i}\right)=0 & (l=1, \ldots, \rho) \\
\Pi^{\alpha}=\Sigma a_{i}{ }^{\alpha} d x^{i}=0, & \left(\alpha=1, \ldots, \rho_{1}\right)
\end{array}
$$

où les $f_{l}$ et $a_{i}^{\alpha}$ sont des fonctions analytiques des coordonnées locales $x^{\prime}$. Toute solution de $S$ est solution dn système fermé $S^{\prime}[14]$ (obtenu en ajoutant aux équations de $S$ le équations dont les premiers $n$ embres cont les diffé rentielles extérieures des premiers membres de $S$ ):

$$
f_{l}=0, \quad \Pi^{x}=0, d f_{l}=0, \quad d \Pi^{x}=0 ;
$$

ce système $S^{\prime}$ peut s'écrire:

$$
\begin{array}{rlrl}
f_{l}\left(\alpha^{i}\right) & =0, & \left(l=1, \ldots, \rho_{0}\right) \\
\theta^{\beta}=0, & \left(\beta=1, \ldots, \rho_{0}+\rho_{1}\right) \\
\varphi^{\alpha}=0, & \left(\alpha=1, \ldots, \rho_{1}\right),
\end{array}
$$

où les $\theta^{\beta}$ sont des formes de Prafr linéairement indépendantes linéaires en $d x^{i}$, les $\varphi^{\alpha}$ étant des formes quadratiques extérieures (égales anx $d \Pi^{\circ}$ ). Nous désignerons par $G_{m}^{h}$ la variété engendrée par tous les éléments de contact de dimension $h(h=1, \ldots, m)$ tangents à la rarieté $V_{m}$. Les équa tions (1.2) définissent une sous-variêté de $V_{m}$ que nous désignerons par $\mathscr{F}^{0}$; nous supposerons $\mathfrak{F}^{0}$ régulière (c'est-à-dire ayant tous ses points régulieıs). Si dans un voisinage $U^{\prime} \subset U$, le rang du systeme des formes $\theta^{\beta}$ a une valeur constante $s_{n}$, les points de $\mathscr{F}^{\circ}$ situés dans ce voisinage sont dits réguliers pour le système de Pfaff $S$; le nombre $s_{0}$ est le caractère d'ordre 0 du système $S$. Un élément de contact linéaire $E^{1}$ intégral de $S$ sera dit ordinaire s'il passe par un point régulier pour le système $S$. Soit $\mathscr{F}^{1}$ la famille de tous ces élé. ments de contact linéaires intégraux ordinaires. Suppocons que dans la variêté $G^{1}$ il existe un voisinage $U_{1}$ d'un elément $\left(E^{1}\right)_{0} \in \mathscr{F}^{1}$ jouissant de ]a propriété suivante: le rang du système linéaire auquel doit satisfaire un élement de contact linéaire pour engendrer avec un élément de contact appartenant à $\mathscr{F}^{4}$ un êlément de contact intégral à deux dimensions possède un rang constant $s_{0}+s_{1}$ pour tout élément de $\mathcal{F}^{1}$ situé dans $U_{1}$; les éléments de contact appartenant à $\mathscr{F}^{1} \cap U_{1}$ sont dits réguliers; si $s_{0}+s_{0}<m-1$, par un élément de contact ordinaire $E^{1}$ passent des éléments de contact intégranx à deux dimensions (appelés eléments ordinaires); le nombre $s$, est le caractère d'ordre $1 \mathrm{du}$ système $S$. On definit de même de proche en proche les caractères $s_{2}, \ldots, s_{h-1}, \ldots$ à partir des familles $\mathscr{F}^{2}, \ldots, \mathscr{F}^{n-1}, \ldots$ d'eléments de contact intégraux ordinaires de dimensions $2, \ldots, h-1, \ldots$. Si $s_{0}+s_{1}+\ldots+s_{h-1}$ $<m-(h-1)$ par un élément de contact régulier $E^{h-1}$ passent des éléments de contact intégraux ordinaires $E^{n}$; le système $S$ est alors dit en involution pour la dimension $h$.

Soit $E^{n}$ un elément de contact intégral ordinaire; on peut lui associer 
au moins une chaine d'écéments de contact $E^{h-1}, \ldots, E^{1}, E^{0}$ intégraux réguliers: $E^{0} \subset E^{1} \subset \ldots \subset E^{h-1} \subset E^{h}$. En utllisant le théorème de CAUCHY-KoWALEWSKI, on démontre le théorème suivant:

Si $E^{h}$ est un élément de contact intégral ordinaire et si $E^{0} \subset E^{1} \subset \ldots \subset E^{h}$ est une chaine attachée $\grave{a} E^{h}$, par $E^{0}$ passe au moins une courbe intégrale Q) $_{1}$ tangente $\grave{a} E^{1}$, par $\mathscr{Q}_{1}$ passe au moins une variété intégrale $\mathscr{Q}_{2}$ tangente $\grave{a}$ $E^{2}, \ldots$, par $\mathscr{Q}_{h-1}$ passe au moins une variété intégrale $\mathscr{Q}_{h}$ tangente $\dot{a} E^{n}$.

Une variété $\mathscr{Q}_{h}$ intégrale du système $S$ est dite générale si tous ses éléments de contact de dimension $h$ sont généraux. L'existence d'une telle variété est démontrée par le théorème précédent (Nous ne considérons que les solutions analytiques).

Soit maintenant sur une variété $V_{n} \times V_{p}$ un système de Pfaff $S$ :

$$
\begin{aligned}
& f_{i}\left(x^{i}, y^{\gamma}\right)=0, \\
& \Pi^{\alpha}=\Sigma\left(a_{i}^{\alpha} d x^{i}+b_{{ }^{\alpha}}{ }^{\alpha} d y^{2}\right)=0,
\end{aligned}
$$

où les $x^{i}$ et les $y^{\lambda}$ sont des coordonnées locales au vuisinage de $x_{0} \varepsilon V_{n}$ et $y_{0} \varepsilon V_{p}$, les $a_{i}^{\alpha}$ et les $b_{\lambda}^{\alpha}$ étant des fonctions analytiques de ces coordonnées. Le système $S$ (ou le système fermé $S^{\prime}$ obtenu à partir de $S$ ) est dit en involution relativement aux variables $x^{i}$ s'il est en involution pour la dimension $n$ et s'il existe des éléments intégraux ordinaires se projetant sur un élément de contact de dimension $n$ tangent à $V_{n}$ (e' est-à-dire dont les équations de definition n'introduisent aucune relation entre les différentielles des variables indépendantes $x^{i}$ ). Pour que le système soit en involution pour la dimension $n$, il faut et il suffit que les caractères $d u$ système vérifient l'inégalité: $s_{0}+s_{1}+\ldots+s_{n-1}<p+1$. Pour $q \mathbf{u}^{\prime}$ il existe des variétés intégrales à $n$ dimensions sur lesquelles les $x^{i}$ soient des variables indépendantes, il faut et il suffit (d'après les propriétés des systèmes linéaires) que le rang par rapport aux $d y^{\prime}$ de chacun des systèmes linéaires définissant les éléments de contact intégranx ordinaires de toutes dimensions soit égal au rang par rapport à l'ensemble des $d x^{i}$ et $d y^{\text {' }}$ du même système. Les rangs par rapport aux $d y^{\lambda}$ de ces systèmes linéaires permettent de définir les caractères réduits $s_{0}^{\prime}, \ldots, s_{n-1}^{\prime}$ introduits par E. CarTan. Pour que le système soit en involution relativement aux variables $x^{i}$, il fant et il suffit que $s_{i}^{\prime}=s_{i}(i=0,1, \ldots, n-1)$.

J. M. Thомas a démontré [45] que les variétés intégrales générales $V_{n}$ d'un système en involution par rapport aux variables $x^{i}$ constituent l'ensemble des solutions d'un système $\Sigma^{1}$ d'equations aux dérivées partielles du premicr' ordre qui, en effectuant au besoin une transformation linéaice sur les coordonnées $x^{i}$, peuvent être résolues par rapport aux dérivées

$$
\frac{\partial y^{\lambda_{i}}}{\partial x^{i}}\left(i=1, \ldots, n ; \lambda_{i}=1, \ldots, m_{i} ; m_{i}=s_{0}+s_{1}+\ldots+s_{i-1}\right) .
$$

Ce système $\Sigma^{1}$ qui est complètement intégrable et complètement régulier est une forme particulière de système orthonome passif défini par RIQuinR [43]. 
Ainsi l'ensemble des solutions générales d'un système de Pfaff en involution relativement aux variables $x^{i}$ est l'ensemble des solutions $d^{\prime}$ 'un système aux dérivées partielles complètement régulier et complètement intégrable.

Inversement considérons un système d'équations aux dérivées partielles $\Sigma^{q}$ d'ordre $q$ qui soit complètement intégrable et complètement régulier; si l'on prend comme fonctions inconnues les $y^{\lambda}$ et leurs dérivées jusqu'a l'ordre $q-1$, le système $\Sigma^{q}$ peat être considéré comme un système du premier ordre définissant une sous-variété $\Pi^{1}$ de $J^{1}\left(V_{n}, J^{q-1}\left(V_{n}, V_{p}\right)\right)$. A ce système du premier ordre correspond un système de PFAFF; ì tout jet appartenant à $\Pi^{i}$ correspond dans $J^{1}\left(V_{n}, J^{q-1}\left(V_{n}, V_{p}\right)\right)$ un élément de contact $E^{n}$ de dimension $n$ se projetant suivant un elément de contact tangent à $V_{n}$ mais cet éément $E^{n} n^{\prime}$ est peat-être pas ordinaire: le système de PFaFF n'est peut-être pas en involution relativement aux variables $x^{i}$.

2. Notion de pseudogroupe de Lio [26].

Soit sur une variéte $V_{n}$ un pseudogroupe de transformations analytiques ( $\left.{ }^{*}\right)$. L'ensemble des jets $j_{m}^{q} f$ (d'ordre $q$ ) correspondant à tous les $f \varepsilon \Gamma$ constitue un sous-ensemble $\mathfrak{g} q(\Gamma)$ de la variété $J^{q}\left(V_{n}, V_{n}\right)$ engendrée par tous les jets d'ordre $q$ de $V_{n}$ dans $V_{n}$.

Si $f$ et $g$ sont deux applications dont le composé est défini, on $a$, en raison de la loi de composition entre jets :

$$
j_{x}^{q}(\mathbf{g} f)=\left(j_{f(x)}^{q} \mathbf{g}\right) j_{\infty}^{q} f
$$

dono $\mathfrak{F}^{q}(\mathrm{l})$ est un groupoîde appelé groupoïde d'ordre $q$ associé à $\Gamma$ [26]; on définit ainsi les groupoïdes associés $\mathscr{F}^{0}(\Gamma), \mathscr{F}^{1}(\Gamma), \ldots, \mathscr{F}^{q}(\Gamma) \ldots$ d'ordres $0,1, \ldots, q, \ldots$. Si $s<q$, tout jet $X^{q} \varepsilon \mathfrak{g}^{q}(\Gamma)$ se projette dans $J^{s}\left(V_{n}, V_{n}\right)$ suivant un jet $X^{s} \& \mathscr{f}^{s}(\Gamma)$; donc toute solution de $\mathscr{F}^{q}(\Gamma)$ (ou application $\varphi$ dont les jets $j_{\mathscr{x}}^{q} \varphi$ appartiennent à $\left.\mathfrak{g}^{q}(\Gamma)\right)$ est solution de $\mathfrak{F}^{s}(\Gamma)$; si en particulier toute solution de $\mathcal{F}(\Gamma)$ est une transformation de $\Gamma$, il en est de même de toute solution de $\mathcal{F}^{q}\left(\mathrm{I}^{\prime}\right)$, ou encore: si $\Gamma$ est l'ensemble des solutions de $\mathfrak{F}^{\mathfrak{s}}(\boldsymbol{\Gamma}), \Gamma$ est egalement l'ensemble des solutions de $\mathfrak{g}^{q}(\Gamma)$. On pent alors donner les definitions suivantes:

DÉFINITION 2.1 - Un pseudogroupe de transformations analytiques $\Gamma$ est dit complet d'ordre $q s^{\prime} i l$ existe des groupoïdes associes à $\Gamma$ tels que $\Gamma$ soit l'ensemble de leurs solutions et si $q$ est l'ordre minimum des groupoïdes jouissant de cette propriété.

(2) $\Phi$ étant l'ensemble des ouverts de $V_{n}$, un pseudogroupe d'automorphismes locaux de $V_{n}$ est un ensemble $\Gamma$ d'homéomorphismes vérifiant les axiomes: $1^{\circ}$ ) tout $f \varepsilon \Gamma$ est un homéomorphisme de $\left.U \subset \Phi \operatorname{sur} f(U) \varepsilon \Phi ; 2^{\circ}\right)$ si $U=U_{i} U_{i}$ et $U_{i} \varepsilon \Phi$, pour qu'un homéomorphisme $f$ défini dans $U$ appartienne à $\Gamma$, il faut et il suffit que sa restriction a chaque $U_{i}$ appartienne a $\Gamma ; 3^{0}$ ) pour tout $U \in \Phi$ l'application identique de $U$ appartient a $\Gamma$; si $f \in \Gamma$, on a: $f^{-1} \varepsilon \Gamma$, si $f \varepsilon \Gamma, f^{\prime} \varepsilon \Gamma$ et si $f f^{\prime}$ est défini, on a: $f f^{\prime} \varepsilon \Gamma$. Voir C. Ehresmann [24]. 
DÉFINITION 2.2. - Un pseudogroupe de transformations analytiques $\Gamma$ est appelé un pseudogroupe de Lie si $\Gamma$ est complet d'ordre $q$ et si le groupoïde associé $\mathfrak{F}^{q}(\Gamma)$ est une sous-variété analytique complètement régulière de $J^{q}\left(V_{n}, V_{n}\right)$, c'està-dire si tout jet $X^{q} \& \mathfrak{F}^{q}(\Gamma)$ admet un voisinage distingue $W$ dans $J^{q}\left(V_{n}, V_{n}\right)$ tel que la composante connexe de $X^{q}$ dans l'intersection $\mathfrak{F}^{q}(\Gamma) \cap W$ soit une sousvariété analytique élémentaire complètement régulière $M^{q}$. En raison même de la définition de $\mathfrak{F}^{q}(\Gamma)$, à tout jet $X^{q} \varepsilon \mathfrak{F}^{q}(\Gamma)$ correspond au moins une solution; donc une sous-variété élémentaire $M^{q} \subset \mathfrak{F}^{q}(\Gamma)$ est définie par un système d'équations aux dérivées partielles $\Sigma^{q}$ qui est complètement intégrable. Supposons $\Sigma^{q}$ mis sous la forme complètement régulière définie dans $\S 1$. Désignons par $U$ (resp. V) l'image de $M^{q}$ dans la projection $\alpha^{q}$ (resp. $\beta^{q}$ ) de $J^{q}\left(V_{n}, V_{n}\right)$ dans $V_{n}$ qui à tout jet fait correspondre sa source (resp. son but). $U$ et $V$ sont des ouverts; soient $x^{1}, \ldots, x^{n} ; y^{1}, \ldots, y^{n}$ des coordonnées locales respectivement dans $U$ et $V$. La projection $M^{0}$ de $M^{q}$ dans $V_{n} \times V_{n}$ peut être représentée par les equations:

$$
y^{\mu_{0}+k}=F^{n}\left(x^{1}, \ldots, x^{n}, y^{1}, \ldots, y^{\mu_{0}}\right) \quad\left(k=1, \ldots, n-\mu_{0}\right)
$$

le rang des $F^{k}$ par rapport à l'ensemble des $x^{i}$ et des $y^{i}$ étant $n-\mu_{0}$; le rang des $F^{k}$ par rapport aux $x^{i}$ est aussi égal a $n-\mu_{0}$, sinon les $y^{j}$ $(j=1, \ldots, n)$ seraient liés par des relations ne contenant pas les $x^{i}$ et ne seraient plus des variables indépendantes. Si l'on fixe le point $x \in U$, les équations (2.2) définissent l'intersection de $V$ et de la classe d'intransitivité de $x$ (e'est-à-dire de l'ensemble de tous les points $z \varepsilon V_{n}$ tels qu'il existe au moins une transformation $f \& \Gamma$ appliquant $x$ sur $z$ ). Considérons l'ensemble des classes d'intransitivité quand $x$ parcourt $U$; si dans l'intersection de $V$ et de chacune de ces classes on fixe un point $(y)_{0}$ en donnant à $y^{1}, \ldots, y^{\text {how }}$ des valeurs. constantes indépendantes de la classe d'intransitivité, les intersections de $U$ et des classes d'intransitivité sont définies par les équations:

$$
F^{k}\left(x^{1}, \ldots, x^{n}\right)=c^{\imath e}, \quad\left(k=1, \ldots, n-\mu_{0}\right) .
$$

Comme le rang des $F^{k}$ par rapport aux $x^{i}$ est $n-\mu_{0}$, on pent choisir les coordonnées locales dans le voisinage $U$ de façon que les équations (2.3) puissent s'écrire $x^{\mu_{0}+k}=c^{t e}\left(k=1, \ldots, n-\mu_{0}\right)$ et les équations (2.2) deviennent: $y^{\mu_{0}+k}=x^{\mu_{0}+k}$. Le système $\Sigma^{q}$ peut alors s'écrire (en omettant les équa. tions qui se déduisent de celles déjà écrites par différentiation totale):

$$
\begin{aligned}
& y^{\mu_{0}+k}=\boldsymbol{x}^{\mu_{0}+k} \\
& \left(k=1, \ldots, n-\mu_{0}\right) \\
& \frac{\partial y^{\lambda}}{\partial x^{1}}=a_{i}^{\lambda}\left(x, y, u_{(1)}\right) \text {, } \\
& \left(\lambda=1, \ldots, \mu_{0}\right) \\
& \frac{\partial u_{(1)}^{\nu}}{\partial x^{i}}=b_{i} v\left(x, y, u_{(i)}, u_{(z)}\right), \\
& \left(v=1, \ldots, \mu_{1}\right) \\
& \frac{\partial u_{(q-1)}^{\ell}}{\partial x^{i}}=c_{i}^{p}\left(x, y, u_{(i)}, \ldots, u_{(q)}\right), \\
& \left(\rho=1, \ldots, \mu_{q-1}\right)
\end{aligned}
$$


les dérivées paramétriques d'ordre $1, \ldots, q$ étant désignées par:

$$
u_{(1)}^{1}, \ldots, u_{(1)}^{\mu_{1}} ; \quad u_{(2)}^{1}, \ldots, u_{(2)}^{\mu_{2}} ; u_{(q)}^{1}, \ldots, u_{(q)}^{\mu_{q}} .
$$

Lorsque le psendogroupe $\Gamma$ est transitif, c'est-a-dire lorsque la classe d'in. transitivité de tout point $x \in V_{n}$ est la variété $V_{n}$ elle-même, la variété $\mathscr{J}^{\prime}(\Gamma)$ peut être identifiée à $V_{n} \times V_{n}$ et l'on a: $\mu_{0}=n$.

La projection de la sous-variété élémentaire $M^{q}$ dans $J^{s}\left(V_{n}, V_{n}\right)$ est une sous-variété elémentaire pour $s=0, \ldots, q-1$ (cf. \$ 1). On peut démontrer que les groupoïdes associés à $\mathbf{F}$ d'ordre inférieur à $q$ sont, de même que ${ }^{\mathfrak{q}}(\Gamma)$, des sous-variétés complètement régulières. (Ces groupờdes sont les projections de $\mathfrak{F}^{q}(\Gamma)$ dans $\left.J^{0}\left(V_{n}, V_{n}\right), \ldots, J^{q-1}\left(V_{n}, V_{n}\right)\right)$. Par contre nous ignorons si les groupoìdes associes à $\Gamma$ d'ordre supérieur à $q$ sont des variétés extraites.

DÉfINITION 2.3. - Un pseudogroupe de Lie I' d'ordre $q$ est dit de type fini, de degré $r$, si toute sous-variété élémentaire $M^{q} \subset \mathfrak{F}^{q}\left(\Gamma^{\prime}\right)$ peut être définie par un système de Mayer-Lie de degré $r$ ou par un système admettant un prolongement de Mayer-Lie complètement intégrable de degré $r$.

Un pseudogroupe de LIE qui n'est pas de type fini est dit de type infini ("groupe infini" de Lie suivant la terminologie de E. Oartav).

Comme les prolongements d'un système de MAYrR-Lir complètement intégrable sont complètement intégrables et complètement réguliers, on peut en dóduire que tous les groupoüdes associés à un pseudogroupe de Lie de type fini, de degré $r$, sont des sous-variétés complètement régulières isomorphes à $J^{r-1}(\Gamma)$ (à tout jet $X^{r-1} \varepsilon \mathfrak{g}^{r-}(\Gamma)$ correspond un jet $X^{r-1+s} \varepsilon \mathcal{F}^{*-1+s}\left(\Gamma^{2}\right)$ et inversement). Le degré d'un pseudogroupe de type fini est supérieur ou égal $\grave{a}$ son ordre: en effet si on avait $r<q$, toute solution de $\mathfrak{g}^{q}(\Gamma)$ serait solution de $\mathfrak{F}^{\prime}(\Gamma)$ d'après $\S 1$ et $\Gamma$ serait complet d'ordre $r$.

Parmi les pseudogroupes de LrE de type fini nous envisagerons les pseudogroupes déduits d'un groupe de Lie [27]; $G$ étant un groupe de transformations de LiE opérant sur une variété $V_{n}$, soit $\Gamma$ l'ensemble de toutes les applications $f$ jouissant de la propriété suivante: $f$ est une application biunivoque d'un ouvert $U$ de $V_{n}$ sur un ouvert $f(U)$ de $V_{n}$ dont la restriction à un voisinage suffisamment petit de tout point de $U$ est la restriction d'une application de $G ; \Gamma$ est un pseudogroupe de LIE (déduit de $G$ ).

Exemples de pseudogroupes de Lie: $1^{\circ}$ ) le pseudogroupe des automorphismes locaux analytiques complexes de l'espace numérique complexe $C^{n}$ (que l'on peut identifier à $R^{2 \eta}$ ) est un pseudogroupe infini d'ordre $1 ; \mathfrak{F}^{4}(\Gamma)$ est une variété élémentaire $M^{1}$ définie par le système d'équations aux dérivées partielles $\Sigma^{1}$ :

$$
\frac{\partial y^{j}}{\partial x^{k}}=\frac{\partial y^{j+n}}{\partial x^{k+n}}, \quad \frac{\partial y^{j}}{\partial x^{k+n}}=-\frac{\partial y^{j+n}}{\partial x^{k}} \quad(j, k=1, . ., n) .
$$

$\left.2^{\circ}\right)$ le pseudogroupe déduit du groupe affine complexe est un psendogroupe d'ordre 2, de degré 2; nne variété élémentaire $M^{2}$ pent etre définie par le 
système $\Sigma^{2}$ :

$$
\begin{array}{rlrl}
\frac{\partial y^{j}}{\partial x^{k+n}} & =-\frac{\partial y^{j+n}}{\partial x^{k}}, & \frac{\partial y^{j}}{\partial x^{k}}=\frac{\partial y^{j+n}}{\partial x^{k+n}}, & (j, k=1, \ldots, n) \\
\frac{\partial^{2} y^{x}}{\partial x^{\beta} \partial x \gamma}=0 & & (\alpha, \beta, \gamma=1, \ldots, 2 n) .
\end{array}
$$

$\left.3^{\circ}\right)$ le pseudogroupe déduit du groupe des déplacements euclidiens de $R^{n}$ est d'ordre 1, de degré 2 ; en effet e'est l'ensemble des solutions du système $\mathrm{V}^{1}$ :

$$
\Sigma_{j} \frac{\partial y^{j}}{\partial x^{k}} \frac{\partial y^{j}}{\partial x^{l}}=\delta_{l}^{k} \quad(k, l=1, \ldots, n)
$$

et par dérivation totale des équations (2.7) et éliminations, on obtient:

$$
\frac{\partial^{2} y^{j}}{\partial x^{k} \partial x^{l}}=0 \quad(j, k, l=1, \ldots, n) .
$$

Le système $\Sigma^{\prime}$ est localement équivalent $\grave{a}$ un système complètement régulier.

Si l'on se borne à des groupoïdes $\mathfrak{g}^{q}(\Gamma)$ qui soient des variétés extraites non nécessairement complètement régulières, on est conduit à la notion de pseudogroupe de LIE au sens large:

DéFINITION 2.2'. - Un pseudogroupe de transformations analytiques $\Gamma$ est appelé un p̉seudogroupe de Lie au sens large si $\Gamma$ est complet d'ordre $q$ et s'il existe un nombre $q^{\prime} \geq q$ tel que le groupoïde associé $\mathfrak{F}^{q^{\prime}}(\Gamma)$ soit une variété extraite de $J^{\prime q^{\prime}}\left(V_{n}, V_{n}\right)$.

Si $\mathfrak{F}^{q^{\prime}}(\boldsymbol{\Gamma})$ est une variété extraite qui n'est pas complètement régulière, $\mathfrak{J}^{q}(\Gamma)$ n'est peut-être pas une variété extraite; e'est pourquoi il y a lieu d'introdaire deux ordres $q$ et $q^{\prime}$, alors $q$ ue pour les pseudogroupes de Lik, si $\mathfrak{F}^{q^{\prime}}(\Gamma)$ est une variété extraite, il en est de même de $\mathfrak{F}^{q}(\Gamma)$.

\section{Prolongements d'un pseudogroupe de Lie. Premier théorème fonda- mental de E. Cartan.}

Soit $\Gamma$ un pseudogroupe de LIE d'ordre $q$ opérant sur une variété $V_{n}$. La projection $\alpha^{q-1}$ de $J^{q-1}\left(V_{n}, V_{n}\right)$ sur $V_{n}$ qui a tout jet $X^{q-1} \varepsilon J^{q-1}\left(V_{n}, V_{n}\right)$ fait correspondre sa source $x$ définit sur $J^{\alpha-1}\left(V_{n}, V_{n}\right)$ une structure fibrée de base $V_{n}$ [26]. Toute transformation $f \varepsilon \Gamma$ appliquani un ouvert $U \subset V_{n}$ sur un ouvert $U^{\prime}=f(U)$ se prolonge en un homéomorphisme $f^{q}$ de $\left(\alpha^{q-1}\right)^{-1} U$ sur $\left(\alpha^{q-1}\right)^{-1} U^{\prime}$, appliquant fibre sur fibre, defini de la manière suivante: $f^{\alpha}$ est l'application qui à tout jet $X^{q-1} \varepsilon J^{q-1}\left(V_{n}, V_{n}\right)$, de source $x \in U$, de but $y$, fait correspondre le jet $X^{\prime q-1}=X^{q-1}\left(j_{x}^{q-1} f\right)^{-1}$, de source $x^{\prime}=f(x)$, de même but $y$. L'ensemble des transformations $f^{q}$ correspondant à toutes les transformations $f$ de $\Gamma$ est un pseudogroupe de transformations analytiques $\left(^{3}\right)$ opérant

(3) Ce pseudogroupe a été introduit par AMaLDI [1]. 
sur la variété $J^{q-1}\left(V_{n}, V_{n}\right)$ et laissant invariant le groupoïde $\mathfrak{g}^{q-1}(\Gamma)$ : en effet si $X^{q-1} \varepsilon \mathfrak{g}^{q-1}(\Gamma)$, comme $\left(j_{x}^{q-1} f\right)^{-1} \varepsilon \mathfrak{F}^{q-1}(\Gamma)$, on a alors: $X^{q-1}\left(j_{x}^{q-1} f\right) \varepsilon \mathfrak{F}^{q-1}(\Gamma)$.

Nous désignerons par $\Gamma^{q}$ la restriction de ce pseudogroupe à la sousvariété $\mathfrak{F}^{q-1}(\Gamma): \Gamma^{q}$ est donc un pseudogroupe de transformations opérant sur la sous-variété $\mathfrak{F}^{q-1}(\mathrm{\Gamma})$.

Soit $M^{q-1}$ la projection dans $J^{q-1}\left(V_{n}, V_{n}\right)$ d'une sous-variété élémentaire $M^{q} \subset \mathfrak{J}^{q}(\Gamma)$ et soit $U$ l'image de $M^{q-1}$ dans $V_{n}$ par la projection $\alpha^{q-i}$. Considérons l'ensemble $\gamma^{q}$ des transformations de $\Gamma^{q}$ laissant invariante la sousvariété élémentaire $M^{q-1}$. A toute application $\mathrm{g} \& \Gamma$ dont les jets d'ordre $q$ appartiennent à $M^{q}$ correspond une application $\varphi$ d'une partie $U_{1}$ de $U$ dans $M^{q-1}$, définie par: $\varphi(x)=j_{x}^{q-1} \mathrm{~g}=X^{q-1}$ où $x \in U_{1}$; le jet du premier ordre $Y=j_{x}^{1} \varphi$ est déterminé par le jet $X^{q}=j_{x}^{q} \mathrm{~g}$, ce qui permet d'identifier $J^{q}\left(V_{n}, V_{n}\right)$ avec un sous-espace de $J^{1}\left(V_{n}, J^{q-1}\left(V_{n}, V_{n}\right)\right.$ [26]. Remarquons que l'application $\alpha^{q-1} \varphi$ est l'application identique de $U_{1}$. Soit $\Phi$ l'ensemble des applications $\varphi$ correspondant à toutes les solutions $\mathrm{g}$ d'un système d'équations aux dérivées partielles $\Sigma^{q}$ définissant la variété $M^{q}$ : $\Phi$ est l'ensemble des solutions $d u$ système $\Sigma^{q}$ considéré comme système du premier ordre sur $J^{\prime}\left(V_{n}, J^{q-1}\left(V_{n}, V_{n}\right)\right)$, ou encore, $\Sigma^{q}$ étant écrit sous la forme (2.4), $\Phi$ est l'ensemble des solutions du système de Pfaff $S^{q}$ :

$$
\begin{array}{lrl}
y^{\mu_{0}+k}=x^{\mu_{0}+k}, & \left(k=1, \ldots, n-\mu_{0}\right) . \\
\theta_{(1)}^{k}=d y^{\lambda}-\Sigma a_{i}^{\gamma}\left(x, y, u_{(1)}\right) d x^{i}=0, & \left(\lambda=1, \ldots, \mu_{0}\right), \\
\theta_{(2)}^{\nu}=d u_{(1)}^{\nu}-\Sigma b_{i}^{v}\left(x, y, u_{(1)}, u_{(2)}\right) d x^{i}=0, & \left(\nu=1, \ldots, \mu_{1}\right), \\
\theta_{(q)}=d u_{(q-1)}-c_{i}^{p}\left(x, y, u_{(1)}, \ldots, u_{(q)}\right) d x^{i}=0, & \left(\rho=1, \ldots, \mu_{g-1}\right) .
\end{array}
$$

Toute application $f^{q} \varepsilon \gamma^{q}$ transforme une application $\varphi \varepsilon \Phi$ en une application $\varphi^{\prime} \& \Phi$ telle que: $\varphi^{\prime}\left(x^{\prime}\right)=f^{q-1} \varphi\left[(f)^{-1}\left(x^{\prime}\right)\right]$; par conséquent $f^{q}$ laisse invariant le système de PFAFF $S^{q}$. Inversement soit $f_{1}^{q}$ une application de $J^{q-1}\left(V_{n}, V_{n}\right)$ dans $\boldsymbol{J}^{q-1}\left(V_{n}, V_{n}\right)$ lajssant invariant le système $S^{q}$; $f_{1}^{q}$ est la projection d'une application $f_{1}^{q+1}$ de $J^{q}\left(V_{n}, V_{n}\right)$ dans $J^{q}\left(V_{n}, V_{n}\right)$ laissant invariante la sousvariéte $M^{q}$; done $f_{1}^{q+1}$ se projette dans $V_{n}$ suivant une solution de $\mathfrak{F}^{g}(\Gamma)$ c' est-à-dire, puisque $\Gamma$ est complet d'ordre $q$, suivant une application $f_{1} \varepsilon \Gamma$ et par conséquent on a: $f_{1}^{q} \varepsilon \gamma^{q}$.

Toute application $f^{q} \varepsilon \gamma^{q}$ transformant un jet de but $y$ en un jet de même but laisse invariantes les $\mu_{0}$ formes $\omega_{(i)}^{\lambda}=\Sigma a_{i}^{\lambda} d x^{i}$ (ainsi que les formes $\omega_{(1)}^{p_{0}+k}=d x^{\mu_{0}+k}$ ) et par suite egalement les formes $d \omega_{(1)}^{i}=\Sigma \omega_{(1)}^{j} \wedge \omega_{j}^{i}$; les formes $\omega_{j}^{i}$ qui peuvent s'écrire: $\omega_{j}^{i}=\Sigma A_{j,}^{i} \theta_{(j)}^{\lambda}+\Sigma B_{j y}^{i} \theta_{(2)}^{\nu}\left(\bmod . \omega_{(1)}^{j}\right)$ sont invariantes mod. $\omega_{(1)}^{j}$; ór le système $S^{q}$ demeurant invariant, les formes $\theta \alpha$ sont reproduites à une combinaison linéaire et homogène près et les formes $\Pi_{j}^{i}=$ $=\Sigma A_{j \lambda}^{i} \theta_{(1)}^{\lambda}+\Sigma B_{j v}^{i} \theta_{(2)}^{\nu}$ sont invariantes. On peut démontrer que parmi ces formes il y a $\mu^{0}+\mu^{1}$ formes linéairement indépendantes $\omega_{(2)}^{1}, \ldots$, $\omega_{(2)}^{\mu_{0}-1}{ }^{\mu_{1}}$. Les formes $d \omega_{(2)}^{2}$ sont invariantes. En poursuivant le raisonnement, on démontre que les 
applications de $\gamma^{q}$ laissent invariantes $n+\mu_{0}+\ldots+\mu_{q-1}=m_{q-1}$ formes de PFAFF linéairement indépendantes (à coefficients analytiques):

$$
\begin{gathered}
d y^{i}, \omega_{(1)}^{\lambda}\left(x, y, u_{(1)}, d x, d y\right), \quad \omega_{(2)}^{\nu}\left(x, y, u_{(1)}, u_{(2)}, d x, d y, d u_{(1)}\right), \ldots, \\
\omega_{(q)}^{p}\left(x, y, u_{(1)}, \ldots, u_{(q)}, d x, d y, d u_{(1)}, \ldots, d u_{(q-1)}\right)
\end{gathered}
$$

les formes $\omega_{(p)}^{2}$ sont linéaires en $d x, d y, d u_{(1)}, \ldots, d u_{(x-1)}$, les coefficients étant fonctions des $x, y, u_{(1)}, \ldots, u_{(p)}$.

Il résulte de l'étude précédente que l'ensemble des applications de $\gamma$ est l'ensemble des solutions du système de PFafF $S^{q}$ :

$$
\begin{aligned}
& x^{\prime \mu_{0}+k}=x^{\mu_{0}+k}, y^{\prime i}=y^{i} \quad\left(k=1, \ldots, n-\mu_{0} ; i=1, \ldots, n\right) \\
& \omega_{(1)}^{\lambda}\left(x^{\prime}, y, u_{(1)}^{\prime}, d x^{\prime}, d y\right)=\omega_{(1)}^{\lambda}\left(x, y, u_{(1)}, d x, d y\right) \text {, } \\
& \ldots \ldots \ldots \ldots \ldots \ldots \ldots \\
& \omega_{(q)}^{\prime}\left(x^{\prime}, y, u_{(1)}^{\prime}, \ldots, u_{(q)}^{\prime}, d x^{\prime}, d y, d u_{(1)}^{\prime}, \ldots, d u_{(q-1)}^{\prime}\right)= \\
& =\omega_{(q)}\left(x, y, u_{(1)}, \ldots, u_{(q)}, d x, d y, d u_{(1)}, \ldots, d u_{(q-1)}\right) \text {. }
\end{aligned}
$$

L'ensemble des solutions du système $\mathfrak{S}^{q}$ est l'ensemble des solutions d'un système d'équations aux dérivées partielles du premier ordre qui peut être mis sous une forme complètement régulière et définit ainsi une sous-variété Glémentaire complètement régulière $N^{1}$ de $J^{1}\left(J^{q-1}\left(V_{n}, V_{n}\right), J^{q-1}\left(V_{n}, V_{n}\right)\right.$ ). Done l'ensemble $\gamma^{q}$ des applications du pseudogroupe $\Gamma^{q}$ laissant invariante la sous-variété élémentaire $M^{n-1}$ est un «noyau» de pseudogroupe de Lie du premier ordre: $\gamma^{q}$ est l'ensemble des solutions de la sous-variété $N^{\prime}$, roisinage des jets du premier ordre de l'application identique de $M^{q-1}$. On pent en déduire que le pseudogroupe $\Gamma^{q}$ est un pseudogroupe de LIE du premier ordre fou pseadogroupe normal suivant la definition donnée par E. CART'AN) d'où le premier théorème fondamental de E. Cartan [12]:

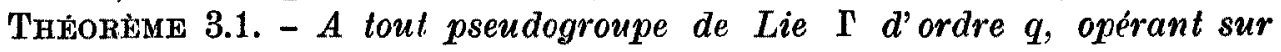
une variété $V_{n}$, est associé un pseudogroupe de Lie $\Gamma^{q}$ du premier ordre cappelé premier prolongement normal de $\Gamma$ ), opérant sur la variété extraite $\mathfrak{g}^{q-1}(\mathbf{\Gamma})$ (de dimension $m_{q-1}$ ), toute application de $\Gamma^{q}$ étant le prolongement d' une application de $\Gamma$; l'ensemble des applications de $\Gamma^{q}$ laissant invariante une sous-variété élémentaire $M^{g-1} \subset \mathfrak{F}^{q-1}([)$ (st caractérisé par la propriété de laisser invariantes $m_{q-1}$ formes de Pfaff linéairemient indépendantes.

Si les groupoirdes associés à $Y$ d'ordre supérieur à $q$ sont des variétés extraites, on peut démontrer de même que les pseudogroupes $\Gamma^{q+1}, \Gamma^{q+2}, \ldots$, $\Gamma^{q+k}, \ldots$ opérant sur les varietés extraites $\mathfrak{f}^{q}(\Gamma), \mathfrak{g}^{q+1}\left(\Gamma^{1}\right), \ldots, \mathfrak{F}^{q+k-1}\left(\Gamma^{q}\right), \ldots$ sont des psendogroupes de LIE $d u$ premier ordre (le prolongement $\Gamma^{q+k}$ est appelé $k^{\text {ieme }}$ prolongement normal de $\Gamma$ ). En particulier si $\mathrm{I}$ est de type fini, de degré $r$, on peut définir ses prolongements normaux successifs qui sont de type fini; nous désignerons par prolongement normal principal de I' le prolongement normal $\Gamma^{r}$ opérant sur la variéte $\mathfrak{g}^{r-1}(\Gamma)$; ce prolongement normal principal est un pseudogroupe de Lie d'ordre et de degré egaux à 1 . 


\section{Equations de structure. Deuxième théorème fondamental de E. Cartan .}

A) Soit $\Gamma$ un pseudogroupe de LIE et $\Gamma^{q}$ son premier prolongement normal. Supposons que le groupoüde $\mathfrak{g}^{q+1}(\Gamma)$ soit une variété extraite définie localement par un système d'équations anx dérivées partielles $\Sigma^{q+1}$ auquel correspondent uu système de PFafF $S^{q+1}$ (appelẹ prolongement du système $S^{q}$ défini par les équations (3.1)) et un système de PFAFF $\mathcal{S}^{q+1}$ (appelé prolon. gement du système $\mathfrak{S}^{q}$ défini par les équations (3.2)). Le système $\mathfrak{S}^{q+1}$ contient les équations de $\mathfrak{S}^{q}$ auxquelles on adjoint $\mu_{q}$ équations:

$$
\begin{aligned}
& \omega_{(q+1)}^{\theta}\left(x^{\prime}, y, u_{(1)}^{\prime}, \ldots, u_{(q+1)}^{\prime}, d x^{\prime}, d y, d u_{(1)}^{\prime}, \ldots, d u_{(q)}^{\prime}\right)= \\
= & \omega_{(q+1)}^{\theta}\left(x, y, u_{(1)}, \ldots, u_{(q+1)}, d x, d y, d u_{(1)}, \ldots, d u_{(q)}\right) .
\end{aligned}
$$

Changeons de notation et désignons par ' $u^{\lambda}$ les dérivées paramétriques d'ordre inférieur ou égal à $q-1$, par $v^{\nu}$ les dérivées paramétriques d'ordre $q$, par $\omega^{j}$ le formes $\omega_{(1)}^{\lambda}, \ldots, \mu_{(q)}^{\lambda}$ et par $\pi^{\alpha}$ les $\mu_{q}$ formes $\omega_{(q+1)}^{p}$; On a : $d \omega^{i}=\Sigma \omega^{k} \wedge \omega^{i}{ }_{k}$, où les formes $\omega^{i}{ }_{k}$ (définies mod. $\omega^{i}$ ) sont linéaires en $d x, d y$, $d u, d v$; en utilisant le même raisonnement que dans $\S 3$, on démontre que les formes $d \omega^{i}$ peuvent s'ecrire:

$$
\begin{aligned}
& d \omega^{i}=\Sigma c_{j k \omega^{j}}^{i} \wedge \omega^{k}+\Sigma a_{j \alpha}^{i} \omega^{j} \wedge \pi^{\alpha}, \quad\left(i, j, k=1, \ldots, m_{q-1}\right) \\
& c_{j k}^{i}+c_{k j}^{i}=0,
\end{aligned}
$$

où les $a_{j \alpha}^{i}$ sont invariants par les transformations de $\Gamma^{q}$. En désignant de manière abregée les formes $\omega^{i}\left(x^{\prime}, y^{\prime}, u^{\prime}, v^{\prime}, d x^{\prime}, d y, d u^{\prime}\right)$ par $\omega^{\prime i}$, on a également:

$$
d \omega^{i}=\Sigma c_{j k}^{i} \omega^{j} \wedge \omega^{\prime k}+\Sigma a_{j \alpha}^{i} \omega^{j} \wedge \pi^{\prime \alpha} .
$$

Le système $\mathfrak{S}^{q+1}$ peut $\mathrm{s}^{\prime}$ écrire :

$$
\omega^{\prime i}=\omega^{i}, \quad \pi^{\prime \alpha}=\pi^{\alpha} \quad\left(i=1, \ldots, m_{q-1}: \alpha=1, \ldots, \mu_{q}\right) .
$$

On en déduit, en tenant compte des relations: $a_{j \alpha}^{i}=a_{j \alpha}^{i}$ :

$$
c_{j k}^{i}=c_{j k}^{i}, \quad\left(i, j, k=1, \ldots . m_{q-1}\right) .
$$

Ces coefficients sont donc invariants par les transformations de $\Gamma^{q}$ laissant invariante la sous-variété élémentaire $M^{q-1}$ et par suite sont des fonctions des invariants $x^{\mu+k}$. Les équations (4.1) sont appelées équations de structure du pseudogroupe normal $\Gamma^{q}$.

Le système de Pfafr $\mathfrak{S}^{q}$ :

$$
\omega^{\prime}-\omega^{i}=0 \quad\left(i=1, . .,, m_{q-1}\right)
$$

(auquel on adjoint les équations $d\left(\omega^{i}-\omega^{i}\right)=0$ ) n'est peut-être pas en invo. lution. Pour qu'il le soit, il faut et il suffit d'après $\S 1$ que les caractères réduits $s_{j}^{\prime}$ de ce système soient égaux aux caractères $s_{j}$; cette condition est équivalente à la suivante: il faut et il suffit que le nombre de paramètres (que nons désignerons par 2 ), dont dépend l'élément intégral ordinaire à 
$m_{q-1}$ dimensions soit à égal à :

$$
s_{1}^{\prime}+2 s_{2}^{\prime}+\ldots+(N-1) s_{N-1}^{\prime}+N \sigma_{N},
$$

où l'on a pose $N=n_{g-1}$ (pour simplifier les notations) et

$$
\sigma_{N}=\mu_{q}+N-\left(s_{0}^{\prime}+s_{1}^{\prime}+\ldots+s_{N-1}^{\prime}\right) \text {. }
$$

Comme le caractère $s_{0}^{\prime}$ est égal an rang des formes $\omega^{\prime i}-\omega^{i}$ e' est-à-dire a $N$, on a: $\sigma_{N}=\mu_{q}-\left(s_{1}^{\prime}+s_{2}^{\prime}+\ldots+s_{N-1}^{\prime}\right)$. Les nombres $s_{1}^{\prime}, s_{1}^{\prime}+s_{2}^{\prime}$, $s_{1}^{\prime}+s_{2}^{\prime}+s_{3}^{\prime}, \ldots$ sont le rangs respectifs des systèmes d' équations :

$$
\begin{aligned}
& \Sigma\left(u^{1} a_{1 \alpha}^{i}+u^{2} a_{2 \alpha}^{i}+\ldots+u^{n} a_{n \alpha}^{i}\right) \pi^{3}=0 \quad(i=1, \ldots, n) \\
& \left\{\begin{array}{l}
\Sigma\left(u^{1} a_{1 \alpha}^{i}+u^{2} a_{2 \alpha}^{i}+\ldots+u^{n} a_{n \alpha}^{i}\right) \pi^{\alpha}=0, \\
\Sigma\left(v^{1} a_{1 \alpha}^{i}+v^{2} a_{2 \alpha}^{i}+\ldots+v^{n} a_{n \alpha}^{i}\right) \pi^{\alpha}=0,
\end{array} \quad(i=1, \ldots, n),\right. \\
& \left\{\begin{array}{l}
\Sigma\left(u^{1} a_{1 \alpha}^{i}+u^{2} a_{2 \alpha}^{i}+\ldots+u^{n} a_{n \alpha}^{i}\right) \pi^{\alpha}=0, \\
\Sigma\left(v^{1} a_{1 \alpha}^{i}+v^{2} a_{2 \alpha}^{i}+\ldots+v^{n} a_{n \alpha}^{i}\right) \pi^{\alpha}=0, \\
\Sigma\left(w^{1} a_{1 \alpha}^{i}+w^{2} a_{2 \alpha}^{i}+\ldots+v^{n} a_{n \alpha}^{i}\right) \pi^{\alpha}=0,
\end{array} \quad(i=1, \ldots, n),\right.
\end{aligned}
$$

où les $u^{i}, v^{i}, w^{i}, \ldots$ sont des coefficients arbitraires tels que le rangs des systèmes $(4.4)_{1},(4.4)_{2},(4.4)_{3}, \ldots$ sont les plus grands possibles.

Le nombre 2) est égal au nombre de coefficients indépendants $t_{k}^{x}$ définis par :

$$
\begin{aligned}
& \pi^{\alpha}=\Sigma t_{k}^{\alpha} \omega^{k} \\
& \Sigma a_{j \alpha}^{i} t_{k}^{\alpha} \omega^{j} \wedge \omega^{k}=0
\end{aligned} \quad\left(\alpha=1, \ldots, \mu_{q} ; i=1, \ldots, N\right)
$$

Lorsque le système $\mathcal{S}^{q}$ est en involution, le système des coefficients $a_{j \alpha}^{i}$ est dit involutif.

$B)$ Soit $\Gamma$ un pseudogroupe de Lre de type fini et $\Gamma^{r}$ son prolongement normal principal. Désignons par $u^{\lambda}$ les dérivées paramètriques d'ordre inférieur ou égal à $r-1$, par $\omega^{j}$ les formes $\omega_{(1)}^{\lambda}, \ldots, \omega_{(r)}^{\lambda}$. Comme il $n^{\prime} y$ a pas de dérivées paramétriques d'ordre $r$ les coefficients des formes $\omega^{j}$ sont fonctions de $x, y, u$ et les coefficients $a_{j a}^{i}$ sont nuls (ces coefficients constituent encore un système involutif). Les équations (4.1) deviennent:

$$
\begin{aligned}
& d \omega^{i}=\Sigma c_{j z}^{i} \omega^{j} \wedge \omega^{k}, \\
& c_{j k}^{i}+c_{k j}^{i}=0,
\end{aligned} \quad\left(i, j, k=1, \ldots, m_{r}\right) .
$$

Ces équations sont les equations de structure de $\Gamma^{r}$. Le système de Pfaff $\mathcal{S}^{r}$ : $\omega^{i}=\omega^{i}\left(i=1, \ldots, m_{r}\right)$ est complètement intégrable. Si le pseudogroupe $\Gamma$ est transitif, il en est alors de meme de $\Gamma^{\prime r}$, restrietion de $\Gamma^{r}$ à la variété $\mathfrak{F}^{\prime r-1}(\Gamma) \subset \mathfrak{F}^{r-1}(\Gamma)$ des jets ayant pour but un point fixe $y_{0} \varepsilon V_{n}$ (le coefficients $c_{j k}^{i}$ sont dans ce cas des constantes) et il existe une transformation et une seule $f^{r} \varepsilon \Gamma^{\prime r}$ appliquant un point arbitraire $X^{r-1} \varepsilon \mathfrak{g}^{\prime \prime-1}(\Gamma)$ sur un point arbi- 
traire $X^{\prime r-1} \varepsilon \mathfrak{F}^{\prime r-1}(\Gamma)$. Nous dirons que $\Gamma^{\prime r}$ est dans ce cas simplement transitif. Le prolongement normal principal joue un rôle analogue à celui du groupe des paramètres dans le cas d'un groupe de transformations de LiF.

$C)$ Sur une variété $V_{n}$, de dimension $n$, la suite $\left(\omega^{1}, \ldots, \omega^{n}\right)$ de $n$ formes de PFAFF linéairement indépendante $\omega^{i}=. \Sigma \lambda_{j}^{i} d x^{j}$ (où les $x^{j}$ sont des coordonnées locales dans le voisinage $U$ d'un point de $V_{n}$ et les $\lambda_{j}^{i}$ des fonctions analytiques de ces coordonnées locales) constitue une forme différentielle du premier ordre $\omega$ [26]: $\omega$ est une fonction continue qui à tout point $x \varepsilon U$ fait correspondre un corepère du premier ordre $\omega_{x}$ d'origine $x$ c'est-à-dire un jet du premier ordre inversible de $V_{n}$ dans l'espace numérique $R^{n}$, de source $x$, de but 0 . Ainsi la suite des formes $\omega^{j}$ définies dans $B$ ) constitue une forme différentielle sur la varieté $\mathfrak{F}^{r-1}(\Gamma)$.

Considérons maintenant la suite $\omega(u)=\left[\omega^{1}(x, u, d x), \ldots, \omega^{n}(x, u, d x)\right]$ où les formes $\omega^{i}(x, u, d x)=\Sigma \lambda_{j}^{i}(x, u) d x$ sont linéairement indépendantes, les $\lambda_{j}^{i}$ étant des fonctions analytiques des coordonnees locales $x^{k}$ et de $\mu$ paramètres $u^{1}, \ldots, u^{\mu}$. Cette suite $\omega(u)$ définit dans l'espace $H^{*}\left(V_{n}\right)$ de tous les corepères du premier ordre sur $V_{n}$ une sous-famille analytique de corepères du premier ordre dont l'origine est dans $U$. Ainsi la suite des formes $\omega^{1}, \ldots, \omega^{m_{q-1}}$ définies dans $A$ ) constitue une sous-famille analytique de corepères sur $\mathfrak{g}^{q-1}(\Gamma)$.

L'étude faite dans $A$ ) et $B$ ) conduit à la réciproque $d u$ deuxième théorème fondamental de E. Cartan:

THÉoRìme 4.1. - Soit sur une variété $V_{n}$ un pseudogroupe de transformations analytiques $\Gamma$ jouissant de la propriété suivante: tout point de $V_{n}$ admet un voisinage distingué $U$ tel que les transformations $f \varepsilon \Gamma$ dont la source et le but (') appartiennent à $U$ constituent l'ensemble des solutions du système de Pfaff

$$
\omega^{i}(x, u, d x)=\omega^{i}\left(x^{\prime}, u^{\prime}, d x^{\prime}\right) \quad(i=1, \ldots, n)
$$

oì les $\omega^{i}(x, u, d x)$ définissent dans $U$ une sous-fannille analytique de corepères du premier ordre. Si le système (4.7) est en involution, c'est-à-dire si les diffé. rentielles d $\omega^{i}$ peuvent s'écrire

$$
\begin{aligned}
& d \omega^{i}=\Sigma c_{j k}^{i} \omega^{j} \wedge \omega^{k}+\Sigma a_{j a}^{i} \omega^{j} \wedge \pi^{x} \\
& c_{j k}^{i}+c_{k j}^{i}=0
\end{aligned} \quad(i, j, k=1, \ldots, n)
$$

(où les $a_{j \alpha}^{i}$ et $c_{j k}^{i}$ sont invariants par les transformations de $\Gamma$, les $a_{j k}^{i}$ constituant un système involutif et les formes $\pi^{*}$ étant définies sur la variété $H^{*}\left(V_{n}\right)$ ), le pseudogroupe $\mathrm{I}$ est un pseudogroupe de Lie du premier ordre. En particulier si les $a_{j x}^{i}$ sont nuls, le pseudogroupe $\Gamma$ est un pseudogroupe de type fni de degré 1 .

(4) Si f est une application $d^{\prime}$ un sous-ensemble $U^{\prime}$ de $V_{n}$ sur $f\left(U^{\prime}\right), U^{\prime}$ est appele la source de $\mathbf{f}$ et $\mathbf{f}\left(U^{\prime}\right)$ le but de $\mathbf{f}[28]$. 
En effet si le système de Pfaff (4.7) est en involution, il résulte de l'étude faite dans $\$ 1$ que l'ensemble des solutions générales de ce système est l'ensemble des solutions d'un système d'équations aux dérivées partielles da premier ordre complètement intégrable et complètement régulier: le pseudogroupe $\Gamma$ est alors un pseudogroupe complet du premier ordre tel que le groupoïde $\mathfrak{g}^{\prime}([)$ soit une variété analytique complètement régulière extraite de $J^{\prime}\left(V_{n}, V_{n}\right)$. Comme on n'a pas démontré que l'ensemble des solutions d'un système d'équations aux dérivées partielles complètement intégrable et complètement régulier est l'ensemble des solutions générales d'un système de Pfaff en involution, le deuxième théorème fondamental de E. Cartan n'est pas démontré non plus sauf dans le cas d'un pseudogroupe de type fini, de degré 1: les conditions énoncées dans le théorème 4.1 (avec $a_{j x}^{i}=0$ ) sont alors nécessaires pour que $\Gamma$ soit un pseudogroupe de Lie de type fini, de degré et $d^{\prime}$ ordre 1. Remarquons que si les $a_{j x}^{i}$ sont nuls, la suite $\omega=\left(\omega^{1}, \ldots, \omega^{n}\right)$ définit dans $U$ une forme différentielle du premier ordre.

Si le système (4.7) n'est pas en involution mais admet des prolongements en involution, l'ensemble des solutions générales de ce prolongement est l'ensemble des solutions d'un système d'équations aux dérivées partielles complètement intégrable mais pas nécessairement complètement régulier: le pseudogroupe $\mathrm{I}$ est un pseudogroupe de Lie au sens large.

\section{Chapitre II.}

\section{Le problème d'équivalence de E. Cartan.}

\section{Définition de l'équivalence loeale des structures infinitésimales.}

L'espace $T\left(V_{n}\right)$ des vecteurs tangents à une variété $V_{n}$, de dimension $n$, $r$ fois différentiable (on suppose $r \geq 3$ ) est un espace fibré de symbole $T\left(V_{n}, R^{n}, L_{n}, H\right)$, le groupe structural $L_{n}$ étant le groupe linéaire homogène de l'espace numérique $R^{n}$ [24]; l'espace fibré principal $H\left(V_{n}\right)$ associé à $T\left(V_{n}\right)$ ou prolongement principal du premier ordre de la variété $V_{n}$ est l'ensemble des isomorphismes de $R^{n}$ sur l'espace tangent $T_{\infty}$ à $V_{n}$ au point $x \in V_{n}$ lorsque $x$ parcourt $V_{n}$. Chacun de ces isomorphismes est un repère du premier ordre sur $V_{n}$. On désigne par structure infinitésimale régulière du pre. mier ordre [26] (que nous appellerons plus brièvement structure infinitésimale régulière car nous n'envisagerons pas de structure infinitésimale d'ordre supérieur) une structure fibrée subordonnée à $T\left(V_{n}\right)$ : cette structure subordonnée a pour symbole $T\left(V_{n}, R^{n}, G, H^{\prime}\right)$, le groupe structural $G$ étant un sous-groupe de $L_{n}$. Si $R^{n}$ est muni d'une structure $s$ subordonnée à sa structure d'espace vectoriel réel, admettant $G$ comme groupe d'automor. phismes, cette structure est transportée par un isomorphisme $h_{x} \varepsilon H^{\prime}\left(V_{n}\right)$ sur une structure bien déterminèe définie dans $T_{\infty}$. A chaque $x$ correspond une 
famille $H_{x}{ }^{\prime}$ de repères distingués (ou repères appartenant à $H^{\prime}\left(V_{n}\right)$ ). On a : $H_{x}{ }^{\prime}=h_{x} G$. Dans le voisinage $U$ d'un point de $V_{n}$, la structure infinitésimale régulière, que nous désignerons par $\mathfrak{s}$, peut être déterminée par une forme différentielle du premier ordre $\omega$, c'est-à-dire une fonction continue qui au point $x \in U$ fait correspondre un isomorphisme $h_{x}{ }^{-1}$ de $T_{x}$ sur $R^{n}$, fou corepère distingué d'origine $x$; l'ensemble $H_{n}{ }^{\prime}$ des corepères distingués d'origine $x$ est alors $H_{x}{ }^{\prime *}=G h_{x}{ }^{-1}$. Cette forme différentielle $\omega$ est encore la suite de $n$ formes de PFaFf $\omega^{1}, \ldots, \omega^{n}$, différentiables, linéairement indépendantes en chaque point $x$; le corepère $h_{x}^{-1}$ (que nous désignerons aussi par $\omega_{x}$ ) peut ètre identifié à la suite $\left(\omega_{\infty}{ }^{1}, \ldots, \omega_{\infty}{ }^{n}\right)$ des restrictions des formes $\omega^{1}, \ldots, \omega^{\prime \prime}$ à $T_{\infty}$; la structure $\mathfrak{s}$ peut être déterminée dans $U$ par toute autre suite $\hat{\omega}$ de $n$ formes de Prafr linéairement indépendantes $\tilde{\omega}^{j}=\Sigma u_{i}^{j} \omega^{i}$ telles que les $u_{i}^{j}$ soient des fonctions différentiables de $x$ et que la matrice $u$ d'éléments $u_{i}^{j}$ dófinisse en chaque point une transformation de $G$.

Sur la variété $V_{n}$ une forme différentielle extérieure tensorielle $\Phi$ de degré $q$ est une fonction qui à tout point $x \in V_{n}$ fait correspondre une application linéaire $\Phi_{x}$ de l'espace des $q$-vecteurs en $x$ dans l'espace des tenseurs de type donné en $x$. A tout corepère $\omega_{x}$ (lequel définit une base dans le dual de $\left.T_{x}\right)$ correspondant des composantes $\left(\Phi_{j k . . .}^{i \ldots}\right)_{x}$ de $\Phi_{x}$. A la forme différentielle $\omega=\left(\omega^{1}, \ldots, \omega^{n}\right)$ définie dans $U \subset V_{n}$ correspondent des formes diffórentielles extérieures $\Phi_{j k \ldots . .}^{i} \ldots$ de degré $q$, c'est-à-dire les fonctions : $x \rightarrow\left(\Phi_{j k . . .}^{i \ldots .}\right)_{x}$. Ces formes peuvent s'ecrire :

$$
\Phi_{j k \ldots . .}^{i \ldots \ldots}=\Sigma A_{j k \ldots \alpha_{1} \ldots \alpha_{q}}^{i \ldots \omega^{\alpha_{1}}} \wedge \ldots \wedge \omega^{\alpha_{q}} \quad(i, j, k=1, \ldots, n),
$$

où la restriction à chaque point $x$ des $A_{j k \ldots z_{1} \ldots x_{q}}^{i}$ sont les composantes d'un tenseur. A la forme differentielle $\tilde{\omega}=\left(\tilde{\omega}^{1}, \ldots, \tilde{\omega}^{n}\right)$ où $\tilde{\omega}^{j}=\Sigma u_{i}^{j} \omega^{i}$ correspondent les formes différentielles extérieures $\tilde{\Phi}_{b c . . .}^{a \ldots .}=\Sigma \tilde{A}_{b c \ldots \beta_{1} \ldots \beta_{q}}^{a \beta_{1}} \wedge \ldots \wedge \omega^{\beta_{q}}$ telles que:

$$
\tilde{\Phi}_{b c \ldots}^{a \ldots}=\Sigma u_{i}^{a} v_{b}^{j} v_{c}^{k} \ldots \Phi_{j \dddot{k} \ldots}^{i \ldots .} \quad(a, b, c=1, \ldots, n),
$$

où les $v_{b}^{j}$ sont les óléments de la matrice $u^{-1}$. On en dóduit:

$$
\tilde{A}_{b c \ldots \beta_{1} \ldots \beta q}^{a \ldots \ldots}=\Sigma u_{i}^{a} v_{b}^{j} v_{c \ldots}^{k} \ldots v_{\beta_{1}}^{\alpha_{1}} \ldots v_{\beta_{q}}^{\alpha q} A_{j k \ldots \alpha_{1} \ldots \alpha_{q}}^{i, \ldots} .
$$

Soient deux variétés $V_{n}$ et $\widehat{V}_{n}$, de dimension $n$, sur lesquelles sont définies respectivement des structures infinitésimales régulières $\mathfrak{s}$ et $\widehat{\mathfrak{s} \text {, de même }}$ groupe structural $G$; un isomorphisme local des deux structures est par définition un homéomorphisme différentiable $\varphi$ d'un voisinage $U$ d'un point de $V_{n}$ sur un ouvert $\widehat{U} \subset \widehat{V}_{n}$, jouissant de la propriété suivante: la structure $\mathfrak{\mathfrak { s }}$ étant déterminée dans $U$ par la forme différentielle $\bar{\omega}$, la structure $\mathfrak{g}$ est déterminée dans $U$ par la forme différentielle $\omega$, transformée de $\widehat{\omega}$ par $\varphi$ (désignée par $\omega=\varphi^{*}(\omega)$ ) et telle que :

$$
\omega_{x}=\widehat{\omega}_{\varphi(x)} j_{x \varphi}^{1} \varphi \text { pour tout } x \varepsilon U
$$


où $j_{x}^{1} \varphi$ désigne, comme dans $\$ 1$, le jet du premier ordre de source $x$ de la fonction $\varphi$. Soient $p$ et $\widehat{p}$ les projections canoniques des espaces fibrés $H^{\prime}\left(V_{n}\right)$ et $H^{\prime}\left(\widehat{V_{n}}\right)$ sur leurs bases respectives; l'isomorphisme local $p$ se pro. longe en un homéomorphisme $\bar{\varphi}$ de $p^{-1}(U)$ sur $\widehat{p}^{-1}(\widehat{U})$, ces voisinages étant respectivement homéomorphes à $U \times G$ et $\widehat{U} \times G$. L'isomorphisme $\varphi$ associe également à toute forme différentielle extérieure tensorielle $\Phi$ définie dans $U$ une forme différentielle extérieure tensorielle $\widehat{\Phi}$ définie dans $\widehat{U}$, de mème degré et de même type. La structure $\widehat{\mathfrak{s}}$ sera dite localement isomorphe (ou localement équivalente) à $\mathfrak{s}$ si pour tout point $\widehat{x} \varepsilon V_{n}$ il existe an isomorphisme local $\varphi$ appliquant un ouvert $U \subset V_{n}$ sur un voisinage $\widehat{U}$ de $\widehat{x}$. Si $\varphi_{1}$ et $\varphi_{2}$ sont deux isomorphismes locaux de $V_{n}$ sur $V_{n}^{\prime}$, l'égalité $\varphi_{1}(x)=\varphi_{2}\left(x^{\prime}\right)$ (où $\left.x \varepsilon V_{n}, x^{\prime} \varepsilon V_{n}\right)$ est équivalente à : $x^{\prime}=\varphi_{12}(x)$ où $\varphi_{12}$ est un automorphisme local de la structure g. Le problème d'isomorphie locale lou problème d'équivalence de E. Cartan) est la recherche des conditions nécessaires et suffisantes pour $q u^{\prime}$ une structure infinitésimale régulière soit localement isomorphe à une autre.

Une structure infinitésimale régulière de groupe structural $G$ est dite intégrable si elle est localement isomorphe à la structure $\mathfrak{s}_{0}$ de ce type déterminée sur $R^{n}$ par la forme différentielle $\omega=d x$ (c'est-à-dire la suite $\left.d x^{1}, \ldots, d x^{n}\right)$. Soit $G$ le sous-groupe du groupe affine à $n$ variables tel $q u e$ le plus grand sous-groupe de $G$ laissant fixe le point $O$ soit le groupe $G$. Désignons par $\Theta$ le psendogroupe de LIE, opérant sur $R^{n}$, déduit (cf. $\$ 2$ ) du groupe $\boldsymbol{G}$ et par $\boldsymbol{\Gamma}$ le pseudogroupe des automorphismes locaux de la structure $\mathfrak{s}_{0}$. On a $\Theta \subset \Gamma$. D'autre part le jet du premier ordre de tout automorphisme local détermine une transformation linéaire appartenant à $\Theta$. On en déduit que le groupoïde $d u$ premier ordre $\mathscr{J}^{1}(\Gamma)$ associé à $\Gamma$ coîncide avec le groupoüde $\mathfrak{J}^{1}(\Theta)$ associé à $\Theta$. Si le pseudogroupe $\Theta$ est complet du premier ordre, les pseudogroupes $\Gamma$ et $\Theta$ sont identiques: les seuls changements de coordonnées locales admissibles sont linéaires.

Une structure infinitésimale régulière est dite isotrope au point $x$ s'il existe un automorphisme local de la structure transformant un corepère d'origine $x$ en un corepère arbitraire de même origine; la structure est dite localement homogène $\mathrm{s}^{\prime}$ il existe un automorphisme local de la structure transformant un point arbitraire $x € U$ en un point arbitraire $x^{\prime} \varepsilon U$; le pseudogroupe des automorphismes locaux de la structure est alors transitif.

\section{Connexions affines associés à une structure infinitésimale. Courbure} et torsion.

Soit sur une variété $V_{n}$ une structure infinitésimale régulière dont le groupe structural $G$ est de dimension $p$; désignons encore par $G$ le sousgroupe du groupe affine dont $G$ est le plus grand sous-groupe laissant fixe le point 0 . 
D'après une définition donnée par C. Ehresmann [25], une connexcion infinitésimale ( $\left.{ }^{5}\right)$ dans l'espace fibré $H^{\prime}\left(V_{n}\right)$ est déterminée par une application II de la varieté $T\left(H^{\prime}\right)$ des vecteurs tangents à $H^{\prime}$ dans l'espace tangent $T_{e}(G)$ à la variété du groupe $G$ en $e$ (élément neutre de $G$ ); cette application est une forme différentielle vectorielle vérifiant les conditions suivantes:

$1^{\circ}$ Si l'on fixe le repère $h \varepsilon H^{\prime}\left(V_{n}\right)$, la restriction de $I I$ aux vecteurs $h+d h$ d'origine $h$ est linéaire.

$$
\begin{aligned}
& 2^{\circ} \Pi(h(u+d u))=u^{-1}(u+d u), \text { où } u \varepsilon G . \\
& \left.3^{\circ} \Pi(h+d h) u\right)=u^{-1} \Pi(h+d h) u .
\end{aligned}
$$

Par conséquent si l'on considère le repère $\tilde{h}=h u^{\top 1}$, on a :

$$
\Pi(\tilde{h}+d \tilde{h})=u \Pi(h+d h) u^{-1}-(u+d u) u^{-1} \text {. }
$$

L'application $I I$ est définie par les furmes différentielles $\omega_{j}^{i}$ sur $T\left(H^{\prime}\right)$ telles qne $\Pi(h+d h)$ soit la transformation infinitésimale du groupe $G$ représentée par :

$$
d x^{i}=\Sigma \omega_{j}^{i}(h+d h) x^{j} \quad(i=1, \ldots, n) .
$$

Le rang des formes $\omega_{j}^{i}$ est $p$; ces formes $\omega_{j}^{i}$ sont donc liées par $n^{2}-p$ relations linéaires

$$
\sum_{i, j} \lambda_{j}^{\alpha i} \omega_{j}^{i}=0 \quad\left(\alpha=1, \ldots, n^{2}-p\right)
$$

où les coefficients $\lambda_{j}^{x i}$ sont constants; en effet les équations (6.4) sont les équations lineaires qui définissent $T_{e}(G)$ comme sous-espace vectoriel de $T_{e}\left(L_{n}\right)$ où $L_{n}$ est le groupe linéaire homogène de $R^{n}$.

Comme $(u+d u) u^{-1}$ est la transformation infinitésimale de $G$ :

$$
d x^{i}=\Sigma d u_{l}^{i} v_{j}^{l} x^{j} \quad(i=1, \ldots, n)
$$

l'application $(h, u) \rightarrow h u^{-1}$ fait correspondre à la forme différentielle rectorielle $\Pi$ la forme différentielle vectorielle $\tilde{\Pi}$, définie dans $H^{\prime} \times G$, à valeurs dans $T_{e}(G)$, de composantes:

$$
\tilde{\omega}_{k}^{l}=\Sigma\left(-d u_{i}^{l} v_{k}^{i}+u_{i}^{l} v_{k}^{j} \omega_{j}^{i}\right)=\Sigma\left(u_{i}^{l} d v_{k}^{i}+u_{i}^{l} v_{k}^{j} \omega_{j}^{j}\right) \quad(l, k=1, \ldots, n)
$$

(où les $u_{i}^{l}$ sont les éléments de la matrice $u$, les $v_{k}^{i}$ les éléments de la matrice $\left.u^{-1}\right)$.

A la connexion définie par l'application II est associée la connexion affine definie par la fonction $\Pi^{\prime}$ qui fait correspondre à tout vecteur $h+d h \varepsilon T\left(H^{\prime}\right)$ la transformation infinitésimale du groupe $\mathbf{G}$ représentée par:

$$
d x^{i}=\omega^{i}(p(h+d h))+\Sigma \omega_{j}^{i}\left(h+d h \mid x^{j} \quad(i=1, \ldots, n),\right.
$$

$p$ désignant encore la projection canonique de $H^{\prime}\left(V_{n}\right)$ sur $V_{n}$; on a :

$$
\omega^{t}(p(h+d h))=\left(p^{*} \omega^{t}\right)(h+d h) \quad(i=1, \ldots, n) .
$$

(5) Pour l'étude des connexions infinitésimales voir egalement S. CharN [19]. 
Nous dirons que la connexion affine $\mathrm{II}^{\prime}$ est definie par les formes $\omega^{t}$ et $\omega_{j}^{i}$. Au vecteur $\tilde{h}+d \tilde{h}$ (où $\tilde{h}=h u^{-1}$ ) est associée la transformation infinitésimale de $\theta$ représentée par:

$$
d x^{l}=\tilde{\omega}^{l}(p(h+d h))+\Sigma \tilde{\omega}_{k}^{l}(h+d h) x^{g} \quad(l=1, \ldots, n)
$$

où $\omega^{l}=\Sigma u_{i}^{l} \omega^{t}$, les $\tilde{\omega}_{k}^{l}$ étant définis par (6.6).

Soit sur une variete $\widehat{V_{n}}$ una structure $\widehat{\mathfrak{s}}$ localement isomorphe à la structure $\mathfrak{s}$ sur $V_{n}$; un isomorphisme local $\varphi$, deux fois differentiable. appliquant le voisinage $U$ d'un point de $V_{n}$ sur $\widehat{U}_{\subset} \widehat{V}_{n}$, associe à toute connexion affine sur $U$ une connexion affine sur $\widehat{U}$; en effet $\varphi$ se prolonge en un homéomorphisme différentiable $\bar{\varphi}$ de $p^{-1}(U)$ sur $\widehat{p}^{-1}(\widehat{D})$ et la connexion affine sur $\widehat{U}$ sera déterminée par les formes $\widehat{\omega^{i}}$ et $\omega_{j}^{i}$ transformées des formes $\omega^{i}$ et $\omega_{j}^{i}$ par l'application $\bar{\varphi}^{-1}$.

Dans $\& 5$, la forme $\omega=\left(\omega^{1}, \ldots, \omega^{n}\right)$ définie dans un ouvert $U$ de $V_{n}$ déterminait une section dans l'espace $H^{\prime *}\left(V_{n}\right)$ des corepères distingués. Nous supposerons $q u^{\prime}$ il en sera de même dans le suite de ce chapitre. Par conséquent les formes $\omega_{j}^{i}$, qui avec les formes $\omega^{i}$, definissent la connexion affine $\Pi^{\prime}$ sont des formes sur $V_{n}$ alors que le formes $\tilde{\omega}_{k}^{l}$ (qui avec les formes $\tilde{\omega}^{l}=\Sigma u_{i}^{l} \omega^{i}$ définissent la même connexion) sont des formes sur $H^{\prime}\left(V_{n}\right)$.

A la connexion affine $\Pi^{\prime}$ sur $V_{n}$ est associée une différentiation covariante. Soit $\Phi$ une forme différentielle exterieure tensorielle (cf. $\S 5$ ) dont les composantes par rapport à la forme différentielle $\omega$ sont $\Phi_{j k \ldots . .}^{i \ldots .}$. Nons allons montrer que les formes $D \Phi_{j k \ldots . .}^{i \ldots \text { definies par: }}$

$$
D \Phi_{j k \ldots}^{i \ldots . .}=d \Phi_{j k \ldots}^{i \ldots . .}+\Sigma \omega_{s}^{i} \wedge \Phi_{j k, . .}^{s_{\ldots} \ldots}-\Sigma \omega_{j}^{h} \wedge \Phi_{h \ddot{k} \ldots}^{i \ldots . .}-\Sigma \omega_{k}^{l} \wedge \Phi_{j i \ldots}^{i \ldots .}+\ldots(i, j, k=1, \ldots, n)
$$

(où $d$ est le symbole de différentation extérieure) sont les composantes rela. tivement à $\omega$ d'une forme différentielle extérieure tensorielle que l'on désigne par $D \Phi$ et qui est appelée la différentielle covariante de la forme $\Phi$ par rapport à la connexion affine $\Pi^{\prime}$.

En effet si $\tilde{\Phi}_{b c \ldots . . .}^{a \ldots}$ sont les composantes de $\Phi$ correspondant à $\tilde{\omega}=\left(\tilde{\omega}^{1}, \ldots, \tilde{\omega}^{n}\right)$ où $\tilde{\omega}^{l}=\Sigma u_{i}^{l} \omega^{i}$ et sí l'on définit les formes $D \tilde{\Phi}_{b c . . .}^{a \ldots .}$ par:

$D \tilde{\Phi}_{b \ldots \ldots}^{a \ldots . .}=d \tilde{\Phi}_{b c \ldots . .}^{a \ldots}+\Sigma \tilde{\omega}_{f}^{a} \wedge \tilde{\Phi}_{b c \ldots}^{f \ldots . .}-\Sigma \tilde{\omega}_{b}^{g} \wedge \tilde{\Phi}_{g c \ldots}^{a \ldots .}-\Sigma \tilde{\omega}_{c}^{h} \wedge \tilde{\Phi}_{b h \ldots}^{a \ldots . .}+\ldots \quad(a, b, c=1, \ldots, n)$, on verifie, en tenant compte de $(6.6)$, les relations:

$$
D \tilde{\Phi}_{b c . . .}^{a \ldots .}=\Sigma u_{i}^{a} v_{b}^{i} v_{c}^{k} \ldots D \Phi_{j k \ldots .}^{i \ldots .} \quad(a, b, c=1, \ldots, n),
$$

et les formes $D \Phi_{j k \ldots . .}^{i \ldots t a n t}$ des formes différentielles extérieures sur $V_{n}$, il en est de même des formes $D \tilde{\Phi}_{b c \ldots . .}^{a \ldots}$ (alors que les formes $d \tilde{\Phi}_{b c \ldots . .}^{a \ldots}$ sont des formes sur $\left.H^{\prime}\left(V_{n}\right)\right)$.

Ainsi la différentielle covariante d'une forme différentielle extérieure tensorielle $\Phi$ de degré $q$ est une forme différentielle extérieure $D \Phi$, de mème type, de degré $q+1$. 
En particulier considérons la forme différentielle extérieure tensorielle (que nous désignerons par le symbole $\stackrel{d}{d} x$ ) qui à tout point $x \in V_{\text {" fait cor- }}$ respondre l'application identique de $T_{x}$. Cette forme $\stackrel{d}{d} x$ a pour composantes (relativement à la forme $\omega$ ) : $\omega^{1}, \ldots, \omega^{n}$. La différentielle covariante $D \dot{d} x$ est donc une forme différentielle tensorielle de degré 2 , appelée torsion de la connexion I'. Ses composantes

$$
\boldsymbol{Q}^{t}=D \omega^{t}=d \omega^{t}+\Sigma \omega_{j}^{i} \wedge \omega^{j} \quad(i=1, \ldots, n)
$$

sont appelées formes de torsion de la connexion $\Pi^{\prime} ;$ si l'on pose $\mathbf{Q}^{\prime}=\mathbf{\Sigma} A_{s t}^{i} \omega^{s} \wedge \omega^{t}$, les $A_{s t}^{i}$ definissent le tenseur de torsion.

I ses formes différentielles extérieures quadratiques

$$
\Omega_{j}^{i}=d \omega_{j}^{i}-\Sigma \omega_{j}^{t} \wedge \omega_{t}^{i} \quad(i, j=1, \ldots, n)
$$

constituent également les composantes (correspondant à la forme $\omega$ ) d'une forme différentielle extérieure tensorielle; en effet à la forme $\tilde{\omega}$ correspondent les formes $\tilde{Q}_{k}^{l}=d \tilde{\omega}_{k}^{i}-\Sigma \tilde{\omega}_{k}^{h} \wedge \pi_{h}^{l}$ vérifiant les relations:

$$
\tilde{\mathbf{Q}}_{k}^{l}=\Sigma u_{i}^{l} v_{k}^{j} \Omega_{j}^{j}
$$

$(k, l=1, \ldots, n)$

ces relations $(6.13)$ se démontrent en utilisant les relations (6.6).

Les formes $\Omega_{j}^{i}$ sont appelées formes de courbure de la connexion $\mathrm{II}^{\prime}$ et si l'on pose $Q_{j}^{i}=\Sigma R_{j l m}^{i} \omega^{l} \wedge \omega^{m}$, les $R_{j l m}^{i}$ definissent le tenseur de courbure de la connexion. Par différentiation extérieure des relations:

$$
\begin{array}{lr}
d \omega^{i}=\Sigma \omega^{j} \wedge \omega_{j}^{i}+Q^{i} & (i=1, \ldots, n), \\
d \omega_{j}^{i}=\Sigma \omega_{j}^{t} \wedge \omega_{i}^{i}+\Omega_{j}^{i} & (i, j=1, \ldots, n),
\end{array}
$$

on obtient les relations suivantes généralisant les identités de Bianchi:

$$
\begin{array}{lr}
d \mathbf{Q}^{i}+\Sigma \boldsymbol{Q}^{j} \wedge \omega_{j}^{i}-\Sigma \omega^{j} \wedge \boldsymbol{Q}_{j}^{i}=0 & (i=1, \ldots, n), \\
d \Omega_{j}^{i}+\Sigma \Omega_{j}^{t} \wedge \omega_{t}^{i}-\Sigma \omega_{j}^{t} \wedge \mathbf{Q}_{t}^{i}=0 & (i, j=1, \ldots, n),
\end{array}
$$

relations qui peuvent s' écrire:

$$
\begin{array}{lr}
D \Omega^{i}=\Sigma \omega^{j} \wedge Q_{j}^{i} & (i=1, \ldots, n), \\
D Q_{j}^{i}=0 & (i, j=1, \ldots, n) .
\end{array}
$$

Donc la différentation covariante $d \mathfrak{n}$ tenseur de courbure n'introduit pas de nouveau tenseur [19] (*).

Pour toute structure infinitesimale régulière, il existe toujours des con. nexion affines [25]. Il peut arriver qu'on puisse imposer d'avance de manière canonique un tenseur de torsion (ce sera le cas de toutes les structures que nous étudierons ultérieurement) mais la connexion affine associée à la struc-

(*) Remarquons que pour la forme différentielle extérieuse tensorielle $\Phi$ de composan.

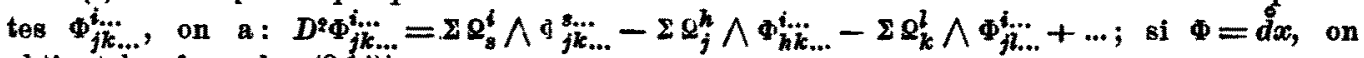
obtient les formules $(6.14)^{\prime}$ 
P. Libermann: Sur le problème d'équivalence, etc.

ture n' est pas nécessairement déterminée (structures presque complexes, presque symplectiques par exemple). Pour certaines structures le choix de la torsion détermine de manière unique la connexion affine associée à la structure (structures presque hermitiennes, presque quaternioniennes par exemple); le groupe structural $G$ est alors tel que les relation (6.4) et $d \omega^{i}-\Omega^{i}=\Sigma \omega^{j} \wedge \omega_{j}^{i}$ déterminent entièrement les $\omega_{j}^{i}$ (on encore les relations (6.4) et $\Sigma \omega^{j} \wedge \omega_{j}^{i}=0$ entrainent $\omega_{j}^{i}=0$ ).

Considérons maintenant une structure infinitésimale régulière (de groupe structural $G)$ qui soit intégrable c' est-à-dire localement isomorphe à la structure $\mathfrak{s}_{0}$ déterminée sur. $R^{n}$ par la forme $d x=\left(d x^{1}, \ldots, d x^{n}\right)$. A la structure $\mathfrak{s}_{0}$ correspond dans $R^{n}$ une connexion naturelle. Tout isomorphisme local d'un ouvert de $R^{n}$ sur un ouvert $U$ de $V_{n}$ associe à cette connexion naturelle une connexion affine dans $U$ que nous appellerons intégrable: cette connexion intégrable peut être définie par des formes $\omega^{i}$ et $\omega_{j}^{i}$ telles que $\omega^{i}=d x^{i}$, $\omega_{j}^{i}=0$; par conséquent, en raison de (6.6), la même connexion est définie par les formes $\tilde{\omega}^{l}=\Sigma u_{i}^{l} d x^{i}$ et $\tilde{\omega}_{k}^{l}=-\Sigma d u_{i}^{l} v_{k}^{i}$. La courbure et la torsion de cette connexion intégrable sont nulles et nous allons montrer que ces propriétés caractérisent une connexion intégrable associée à une structure intégrable. En effet si la courbure est nulle, le système

$$
\tilde{\omega}_{k}^{l}=\Sigma\left(u_{i}^{l} d v_{k}^{i}+u_{i}^{l} v_{k}^{j} \omega_{j}^{i}\right)=0
$$

est complètement intégrable; en raicon des conditions (6.1) les vecteurs tan. gents aux variêtés intégrales ne penvent être tangents aux fibres de $H^{\prime}\left(V_{n}\right)$, donc chaque variêté intégrale de (6.16) définit une section de $H^{\prime}\left(V_{n}\right)$, e'est$a$ dire une fonction contin $a$ qui à tout $x \in V_{n}$ fait correspondre un corepère $\tilde{\omega}_{x}=\left(\tilde{\omega}_{x}^{1}, \ldots, \tilde{\omega}_{x}^{n}\right)$ tel que: $\omega^{i} \omega^{i}=\Omega^{i}(i=1, \ldots, n)$. Si de plus la torsion est nulle, on a $d \tilde{\omega}^{i}=0 \quad(i=1, \ldots, n)$ et il existe un système de coordonnées locales $x^{1}, \ldots, x^{n}$ telles que $d x^{i}=\tilde{\omega}^{t}$. La structure considérée est donc inté. grable et la connexion associée, définie par les formes $d i c^{i}$ et $\tilde{\omega}_{j}^{i}=0$ est intégrable; $d$ ' où :

THÉoRìme 6.1. - Si parmi les connexions affines que l'on peut associer $\grave{a}$ une structure infinitésimale régulière $\mathbf{s}$, il en existe une à courbure et torsion nulles, la structure $\mathfrak{s}$ est intégrable et la connexion considérée est intégrable.

Remarque: suivant une définition donnée par C. EHresmann, une connexion infinitésimale $\Pi^{\prime}$ dans $H^{\prime}\left(V_{n}\right)$ est dite intégrable si le champ $\bar{C}$ transversal aux fibres déterminé par la connexion est complètement intégrable; pour qu'il en soit ainsi, il faut et il suffit que la courbure et la torsion de connexion soient nulles [25]. Donc les connexions intégrables que nous avons définies sont aussi des connexions dont le champ $\bar{C}$ transversal aux fibres est complètement intégrable.

Soit sur une variété $V_{n}$ une struoture infinitésimale régulière intégrable $\mathfrak{g}$ et soient $\varphi_{1}$ et $\varphi_{2}$ deux isomorphismes locaux deux fois differentiables dont 
les sources appartiennent à $R^{n}$, les buts de ces isomorphismes etant respectivement des ouverts $U_{1}$ et $U_{2}$ de $V_{n}$; designons par $\varphi_{12}$ l'automorphisme local de $R^{n}$ tel que $\varphi_{1}(x)=\varphi_{2}\left(x^{\prime}\right)$ soit equivalent à $x^{\prime}=\varphi_{12}(x)$ ò $x \approx R^{n}$, $x^{\prime} \varepsilon R^{n}$. A la connexion naturelle dans $R^{n}$ sont associées par $\varphi_{1}$ et $\varphi_{2}$ deux connexions intégrables dans $U_{1} \cap U_{2}$. Pour que ses connexions colncident, il faut et il suffit que $\varphi_{12}$ appartienne au pseudogroupe $\theta$ déduit du groupe affine $G$ (dont le groupe structural $G$ de $\mathfrak{s}$ est le plus grand sous-groupe laissant fixe le point $O$ ). En effet dans $U_{1} \cap U_{2}$ la première connexion peut ètre définie par les formes $d x^{i}$ et $\omega_{j}^{i}=0$, on encore par les formes $d x^{\prime}=\Sigma u_{\xi}^{l} d x^{l}$ (où les $u_{i}^{l}$ définissent en chaque point une transformation $u$ bien déterminée de $G$ ) et $\tilde{\omega}_{k}^{l}=-\Sigma d u_{i}^{l} v_{k}^{i}$ (les $v_{k}^{i}$ définissant la transformation $u^{-1}$ ); la deuxième connexion peut etre définie par lès formes $d x^{l}$ et $\tilde{\omega}_{k}^{l}=0$; les deux connexions coïncident si les $u_{i}^{l}$ sont des constantes. Par conséquent si l'on ne peut associer qu' une senle connexion affine intégrable à la struc. ture $\mathfrak{s}$, cette structure est localement équivalente à une structure $\mathcal{S}_{0}$ de $R^{n}$ dont le pseudogroupe des automorphismes locaux est le pseudogroupe $\theta$.

Pour toutes les structures infinitésimales régulières que nous étudierons ultérieurement, nous imposerons de manière canonique un tenseur de torsion de façon que la torsion de toute structure intégrable soit nulle: pour ane structure intégrable $\mathfrak{s}$, parmi les connexions affines compatibles avec ce choix de la torsion se trouvent toutes les connexions intégrables que l'on peut associer à cette structure; si de plus le groupe structural $G$ est tel que le choix de la torsion détermine de manière unique une connexion affine, on ne peut alors associer à la structure intégrable $\mathfrak{s}$ qu'une seule connexion affine intégrable et l'on obtient le théorème suivant:

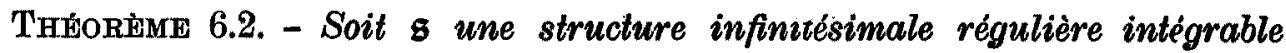
dont le groupe structural $G$ est tel que pour toute structure infinitésimale régulière de même groupe structural, le choix de la torsion détermine canoni. quemenl une connexion affine associée. Le pseudogroupe des automorphismes locaux de la structure $\boldsymbol{s}_{0}$ de même type sur $R^{n}$ (à laquelle $\mathfrak{g}$ est localement équivalente) est alors le pseudogroupe $\Theta$ déduit du groupe affine $\mathrm{G}$ (dont $G$ est le plus grand sous-groupe laissant fixe le point 0 ).

Par conséquent si une structure intégrable $s$ est localement équivalente a une structure $\mathfrak{g}_{0}$ de $R^{n}$ dont le pseudogroupe des automorphismes locaux est un psendogroupe de LIE de type infini, pour aucune structure infinitésimate régulière de même groupe structural, le choix de la torsion ne peut déterminer de manière unique une connexion affine (structures presque complexes, presque symplectiques).

Remarques: $\left.1^{\circ}\right)$ pour qu' une structure infinitésimale régulière telle que le choix de la torsion détermine canoniquement une connexion affine associée soit intégrable, il faut et il suffit que la courbure et la torsion de cette connexion canonique soient nulles. Cette proposition résulte du théorème 6.1. 
$2^{\circ}$ ) La torsion étant déterminée canoniquement, tout automorphisme local d'une structure infinitésimale régulière laisse invariantes les composantes $A_{s t}^{i}$ du tenseur de torsion. Donc si la structure est isotrope en tout point $x \mathrm{~d}^{\prime}$ un voisinage $U$, les $A_{s t}^{i}$ sont en chaque point indépendantes $\mathrm{du}$ corepère distingué. Si, de plus, la structure est localement homogène dans $U$, les $A_{s t}^{i}$ sont des constantes.

Lorsque le choix de la torsion détermine canoniquement une connexion affine associée, on obtient les mêmes résultats pour les composantes $R_{j l m}^{i}$ du tenseur de courbure.

\section{Problème d'équivalence restreint [13].}

Le problème d'équivalence restreint est relatif à des structures infinitésimales régulières $\mathfrak{s}$ et $\widehat{\mathfrak{B}}$ (définies respectivement sur des variétés $V_{n}$ et $\widehat{V_{n}}$ ) dont le groupe structural $G$ est réduit à la transformation identique, ce qui correspond à un parallélisme sur $V_{n}$ et $\widehat{V_{n}}$.

La structure $g$ (resp. $\widehat{g})$ étant définie dans le voisinage $U$ d'un point de $V_{n}$ (resp. le voisinage $\widehat{U}$ d'un point de $V_{n}$ ) par la suite de $n$ formes de PFafF linéairement indépendantes $\omega^{t}(x, d x)$ (resp. $\left.\widehat{\omega^{i}(x,} d \widehat{x}\right)$, linéaires par rapport aux différentielles des coordonnées locales $x^{1}, \ldots, x^{n}$ (resp. $\widehat{x^{1}}, \ldots, \widehat{x^{n}}$ ), les coefficients étant fonctions de ces coordonnées, un isomorphisme local de $U$ dans $\widehat{U}$ est un homéomorphisme différentiable solution du système de Pfaff:

$$
\omega^{i}(x, d x)=\widehat{\omega^{i}}(\widehat{x}, d \widehat{x}) \quad(i=1, \ldots, n) .
$$

De ces équations on déduit:

$$
d \omega^{i}(x, d x)=\widehat{d \omega^{i}(\widehat{x}, d \widehat{x})} \quad(\vec{i}=1, \ldots, n) .
$$

Les différentielles $d \omega^{i}$ peuvent s'écrire :

$$
d \omega^{i}=\Sigma c_{j k}^{i}(x) \omega^{h} \wedge \omega^{h}
$$

de même on a :

$$
\widehat{d \omega^{i}}=\sqrt{c_{j k}^{i}} \widehat{(x)} \widehat{\omega^{j}} \wedge \widehat{\omega^{k}} .
$$

En raison $d u$ théorème 4.1 , le pseudogroupe des automorphismes locaux de chacune des structures $\mathfrak{s}$ et $\widehat{\mathfrak{s}}$ est un psendogroupe de LIE de type fini d'ordre et de degré égaux à 1.

Supposons d'abord les $c_{j k}^{i}$ constants : le pseudogroupe des automorphismes locaux de la structure $\mathfrak{s}$ est simplement transitif; pour que la structure $\widehat{\mathfrak{s}}$ soit localement équivalente à $\mathfrak{s}$, il faut et il suffit que

$$
c_{j k}^{i}=\widehat{c_{j k}^{i}} \quad(i, j, k=1, \ldots, n)
$$

car alors le système (7.1) est complètement intégrable; le pseudogroupe des automorphismes locaux de la structure $\widehat{\mathfrak{z}}$ est également simplement transitif. Tout point $x \in U$ peut ëtre appliqué sur un point arbitraire $\widehat{x} \varepsilon \widehat{U}$. 
Lorsque les coefficients $c_{j k}^{i}(x)$ ne sont pas de constantes, en obtient par differentiations successives :

$$
\begin{aligned}
& d c_{j k}^{i}=\Sigma c_{j k \mid l \omega^{l}}^{i}, \\
& d c_{j k \mid l}^{i}=\Sigma c_{j k \mid l m}^{i} \omega^{m}, \\
& \ldots \ldots \ldots \ldots \\
& d c_{j k \mid l_{1} \ldots l_{q}}^{i}=\Sigma c_{j k \quad l_{1} \ldots l_{q+1}}^{i} \omega^{l_{q+1}} .
\end{aligned}
$$

Les fonctions $c_{j k \mid l_{1} \ldots l_{g}}^{i}(x)$ sont invariantes par tout automorphisme local de la structure s. Soit $p$ le plus petit nombre tel que toutes le fonctions $c_{j k \mid l_{1} \ldots l_{p} \ldots l_{q}}^{i}$ (où $q>p$ ) soient des fonctions des $c_{j k \mid l_{1} \ldots l_{h}}^{i}(h \leq p)$; soit $n_{1}$ le nombre des $c_{j k \mid h_{1} \ldots l_{h}}^{i}$ qui sont indépendantes (c'est-à-dire dont les différentielles sont linéairement indépendantes). On définit de mème des invariants $\widehat{c_{j k \mid l_{1} \ldots l_{q}}}$ pour la structure $\widehat{\mathbb{B}}$; pour que la structure $\widehat{\mathbf{g}}$ soit localement équivalente $\grave{a}$ la structure $s$ il faut que les invariants de mêmes indices soient égaux pour les deux structures; en particulier les nombres $p$ et $n_{1}$ doivent ètre les mêmes.

Si $n_{1}=n$, le pseudogroupe des automorphismes locaux de chacune des deux structures est le pseudogroupe des applications identiques des ouverts de $V_{n}$ ou $\widehat{V}_{n}$. Toute solution de (7.1) est solution du système

$$
\begin{aligned}
& c_{j k}^{i}(x)=\widehat{c_{j k}^{i}(\widehat{x}),} \\
& c_{j k}^{i}(x)=\widehat{c_{j k}^{i}(x)}, \\
& \cdots \cdots \cdots \\
& c_{j k \mid l_{1} \ldots l_{p}}^{i}(x)=\widehat{c_{j k}^{i}} \mid l_{1} \ldots l_{p}(\widehat{x}), \\
& c_{j k \mid l_{1} \ldots l_{p+1}}^{i}(x)=\widehat{c_{j k}^{i} \mid l_{1} \ldots l_{p+1}} \widehat{(x)},
\end{aligned}
$$

et inversement toute solution de (7.5) est solution de (7.1). En effet si les équations (7.1) sont satisfaites, on obtient les équations $(7.5)$ par différentiations successives. Inversement si l'on prend dans $U$ (resp. $\widehat{U}$ ) des coordonnées locales $x^{1}, \ldots, x^{n}$ (resp. $\widehat{x^{1}}, \ldots, \widehat{x^{n}}$ ) fonctions de $c_{j k \mid l_{1} \ldots l_{h}}^{i}$ (resp. $\widehat{c_{j k}^{i} \mid l_{1} \ldots l_{h}}$ ) où $h \leq p$, les équations (7.5) penvent s'écrire (en posant $\left.d x^{i}=\Sigma \lambda_{j}^{i} \omega^{j}, \widehat{d x^{i}}=\Sigma \bar{\lambda}_{j}^{i} \omega^{j}\right)$ : $x^{i}=\widehat{x^{i}}, \ldots, x^{n}=\widehat{x^{n}}, \widehat{\lambda_{j}^{i}}=\widehat{\lambda_{j}^{i}}(i, j=1, \ldots, n)$. Par conséquent, le rang de la matrice $\lambda_{j}^{i}$ (resp. $\widehat{\lambda}_{j}^{i}$ ) étant $n$, si les equations (7.5) sont satisfaites, il en est de même des équations (7.1): Ces condition réalisées, tout point $x \& U$ est applicable sur un point bien déterminé $\bar{x} \varepsilon \widehat{U}$.

Si $n_{1}<n$, toute solution de (7.1) est encore solution de (7.5). Prenons dans $U$ (resp. $\widehat{U}$ ) un système de coordonnées locales $x^{1}, \ldots, x^{n}$ (resp. $\widehat{x^{1}}, \ldots, \widehat{x^{n}}$ ),

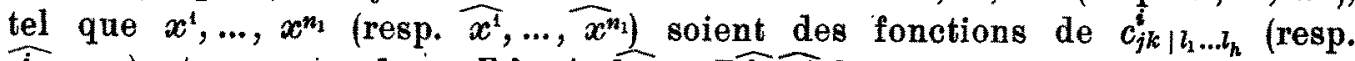
$\left.\widehat{c_{j k \mid}^{i} \mid l_{1} \ldots l_{h}}\right)$ et posons : $d x^{\alpha}=\Sigma \lambda_{i}^{\alpha} \omega^{t}, \widehat{d x^{\alpha}}=\Sigma \widehat{\lambda_{i}^{\alpha} \omega^{t}}$, les indices grecs variant de 1 
P. Libermann: Sur le problème d'équivalence, ete.

à $n_{1}$, les indices latins de 1 à $n$. Pour toute solution de (7.5), on a: $\widehat{x}=x$, $\widehat{\lambda_{i}^{\alpha}}=\lambda_{i}^{\alpha}\left(\alpha=1, \ldots, n_{1}, i=1, \ldots, n\right)$. Done si l'on remplace dans (7.1) les $\widehat{x}$ par $x$, on obtient un système de PFaFF $\omega^{i}-\widehat{\omega^{i}}=0(i=1, \ldots, n)$ tel que le rang des formes $\omega^{i}-\widehat{\omega^{i}}$ (linéaires par rapport aux différentielles de $2 n-n_{1}$ variables $\left.x^{1}, \ldots, x^{n}, \widehat{x^{n_{1}+1}}, \ldots, \widehat{x^{n}}\right)$ soit $n-n_{1}$. On a:

$$
\begin{aligned}
d\left(\omega^{i}-\widehat{\omega^{j}}\right)=\frac{1}{2} \Sigma c_{j k}^{i}\left(x^{x}\right)\left[\left(\omega^{j}-\widehat{\omega^{j}}\right) \wedge\left(\omega^{k}-\widehat{\omega^{k}}\right)+\left(\omega^{j}+\widehat{\omega^{j}}\right) \wedge\left(\omega^{k}-\widehat{\omega^{k}}\right)\right] \\
(i=1, \ldots, n) ;
\end{aligned}
$$

le systéme (7.1) devient done complètement intégrable.

Le pseudogroupe des automorphismes locaux de la structure $\gamma$ et de la structure $\widehat{\gamma}$ qui lui est localement équivalente est un psendogroupe de LIE de type fini admettant $n_{1}$ invariants.

\section{Problème d'équivalence général.}

A) Structures pour lesquelles le choix de la torsion ne détermine par canoniquement une connexion affine associée.

Nous supposons déterminée canoniquement la torsion (ce qui est possible pour toutes les structures étudiées ultérieurement). Nous supposons, de plus, dans ce paragraphe les données analytiques.

Soit sur une variété $V_{n}$, une structure infinitésimale régulière $\mathfrak{s}$, de groupe structural $G$, déterminée dans le voisinage $U$ d' un point de $V_{n}$ par une forme différentielle $\omega=\left(\omega^{1}, . ., \omega^{n}\right)$, les différentielles $d \omega^{t}$ vérifiant les relations $(6.11)^{\prime}$ :

$$
d \omega^{i}=\Sigma \omega^{j} \wedge \omega_{j}^{i}+\Omega^{i}
$$

où les formes $\Omega^{i}=\Sigma A_{s t}^{i} \omega^{s} \wedge \omega^{t}$ sont les formes de torsion. Aux formes $\tilde{\omega}^{l}=\Sigma u_{i}^{l} \omega^{i}$ définissant la même structure, correspondent les formes $\tilde{\mathbf{Q}}^{l}=\tilde{A}_{k h}^{l} \tilde{\omega}^{k} \wedge \pi^{h}$ telles que: $\tilde{A}_{k h}^{l}=\Sigma u_{i}^{l} v_{s}^{k} v_{t}^{h} A_{s t}^{i}$ (cf. formules (5.3)).

Lorsqu'en chaque point $x \in \bar{U}$, les composantes $\tilde{A}_{k h}^{l}$ du tenseur de torsion ne sont pas toutes indépendantes du corepère, on peut choisir en tout point $x \varepsilon U$, dans la famille $H_{x}^{\prime *}$ des corepères distingués d'origine $x$, une sousfamille distinguée $H_{x}^{(1) *}$ telle pour chacun des corepères appartenant à $H_{x}^{(1) *}$, le plus grand nombre possible de composantes $\tilde{A}_{k h}^{l}$ prennent une valeur donnée indépendante de $x$ (on choisit le plus grand nombre possible de com. posantes nulles); les composantes qui ne peuvent prendre des valeurs arbitraires ont des valeurs indépendantes du choix au corepère appartenant à $H_{x}^{(1) *}$ : ces composantes sont des fonctions de $x$, invariantes par tout automorphisme local de la structure. Lorsque les composantes $\tilde{A}_{k h}^{l}$ du tenseur de torsion sont en tout point $x \in U$ indépendantes du corepère appartenant à $H_{x}^{*}$ mais ne sont pas toutes des constantes, on peut encore choisir une sous-famille distinguée $H_{x}^{(1) *}$ de la manière suivante: les différentielles $d \tilde{A}_{k h}^{l}$ sont des formes invariantes par les automorphismes locaux de la structure; 
l'une d'elles peut s'écrire: $\varphi=\Sigma a_{i} \omega^{i}=\Sigma \tilde{a}_{l} \tilde{\omega}^{l}$ avec $\tilde{a}_{l}=\Sigma v_{i}^{i} a_{i}$ et les $\tilde{a}_{l}$ sont donc les composantes d'un tenseur; on détermine la sous-famille $H_{x}^{(1) *}$ en donnant aux composantes des differents tenseurs correspondant a toutes les formes invariantes le plus grand nombre possible de valeurs constantes arbitraires.

On associe canoniquement à la famille $H_{x}^{(1) *}$ de nouveaux tenseurs (en considérant par exemple des formes de PFAFF invariantes). Par la même méthode que précédemment, on distingue en chaque point $x \varepsilon U$ une sousfamille $H_{x}^{(2) *} \subset H_{x}^{(1) *}$. En poursuivant l'application de cette methode, on obtient en chaque point $x$ des sous-familles $H_{x}^{(1) *} \supset H_{x}^{(2) *} \supset \ldots \supset H_{x}^{(r) *}$ et les opérations se terminent lorsque l'une des conditions suivantes est réalisée:

$\left.1^{\circ}\right)$ la sous-famille $H_{x}^{(r) *}$ se réduit en chaque point à un seul corepère distingué et l'on est ramené au problème d'équivalence restreint. Tout iso. morphisme local de $U$ sur un ouvert $\widehat{U}$ d'une variété $\widehat{V_{n}}$ applique le corepère distingué d'origine $x \varepsilon U$ sur un corepère distingué bien déterminé $d$ 'origine $x \subset \widehat{U}$.

$\left.2^{\circ}\right)$ les composantes de tout tenseur que l'on peut associer canoniquement à la sous-famille $H_{x}^{(r) *}$ sont indépendantes du corepère appartenant à $H_{x}^{(r) *}$; il existe alors un automorphisme local de la structure $g$ transformant un corepère arbitraire $h_{x}^{-1} \varepsilon H_{x}^{(r) *}$ en un corep̀̀re arbitraire $h_{x}^{\prime-1} \varepsilon H_{x}^{(r) *}$. Lorsque $x$ parcourt $U, H_{x}^{(r) *}$ engendre une sous-famille analytique de corepères définie par des formes $\tilde{\omega}^{j}(x, u, d x)$ dépendant de paramètres $u^{1}, \ldots, u^{q}$. Les différentielles $d \tilde{\omega}^{i}$ peuvent s'écrire:

$$
d \tilde{\omega}^{t}=\Sigma c_{j k}^{i} \tilde{\omega}^{j} \wedge \tilde{\omega}^{k}+\Sigma a_{j a}^{i} \tilde{\omega}^{j} \wedge \Pi^{a} \quad(i=1, \ldots, n)
$$

où les formes $\Pi^{\alpha}$ (définies mod. $\omega^{j}$ ), linéaires en $\tilde{\omega}^{\alpha}$ et $d u^{l}$ sont linéairement indépendantes par rapport aux $d u^{i}$; les coefficients $c_{j k}^{l}, a_{j \alpha}^{i}$ sont invariants par les automorphismes locaux de la structure s. Si les $a_{j \alpha}^{i}$ forment un système involutif, en raison du théorème 4.1, le pseudogroupe des automorphismes locaux de la structure $s$ est un pseudogroupe de Lie du premier ordre (il en est de même du pseudogroupe des automorphismes locaux de toute structure $\widehat{\mathfrak{g}}$ localement équivalente à la structure s). Si les $a_{j \alpha}^{i:}$ ne forment pas un système involutif, on prolonge le système $\tilde{\omega}^{j}=\widehat{\omega}^{j}$. en lui adjoignant les équations $\Pi^{\alpha}=\widehat{\Pi}^{\alpha}$. Si le nouveau système n'est pas en involution, on en considère des prolongements successifs; dans tous les exemples que nons étudierons, on obtient finalement un système en involution et d'après une remarque de $\S 4$, le pseudogroupe des automorphismes locaux de la structure $g$ est alors un pseudogroupe de Lie au sens large.

Lorsque nous appliquerons cette méthode, nous nous trouverons toujours dans le cas suivant: le sous-familles $H_{x}^{(1) *}, \ldots, H_{x}^{(r) *}$ définissent des structures infinitésimales régulières $\mathfrak{s}^{(1)}, \ldots, \mathfrak{g}^{(r)}$, chacune subordonnée à la précédente et la structure $\mathcal{G}^{(r)}$ est done isotrope. Considérons en effet la sous-famille $H_{\infty}^{(1) *}$ 
par exemple; en tenant compte de ce que toutes les composantes de tenseurs associés à $H_{x}^{(1) *}$ ont des valeurs indépendantes du corepère appartenant à $H_{x}^{(1) *}$ et en utilisant les relations (5.3), on démontre que la transformation $u$ de $G$ qui transforme le corepère $h_{x}^{-1} \varepsilon H_{x}^{(1) *}$ en le corepère $h_{x}^{\prime-1} \varepsilon H_{x}^{(1) *}$ laisse invariante la sous-famille $H_{x}^{(1) *}$; par conséquent, on a: $H_{x}^{(1)}=G_{x}^{(1)} h_{x}^{-1}$, où $G_{x}^{(1)}$ est un sous-gruupe de $G$. Lorsque $G_{x}^{(1)}$ ne depend pas de $x$, on a quel que soit $x: H_{x}^{(1)}=G^{(1)} h_{x}^{-1}$ et la sous-famille $H_{x}^{(1) *}$ définit une structure infinitesimale réguliere, de groupe structural $G^{(1)}$.

B) Structures auxquelles on peut associer canoniquement une connexion affine.

La restriction à chaque repère $\hat{h}_{x} \varepsilon H^{\prime}\left(V_{n}\right)$ de l'ensemble des formes $p^{*} \tilde{\omega}^{l}$ (transformées des formes $\tilde{\omega}^{l}$ par la projection $p^{\prime}$ de $H^{\prime}\left(V_{n}\right)$ sur $V_{n}$ ) et $\tilde{\omega}_{k}^{l}$ définit un corepère sur la variêté $H^{\prime}\left(V_{n}\right)$ : à chaque $\tilde{h}_{x}$ correspond canoniquement un seul corepère sur $H^{\prime}\left(V_{n}\right)$ et l'on est ramené au problème d'équivalence restreint; en raison des relations $(6.11)^{\prime}$ et $(6.12)^{\prime}$ les composantes des tenseurs de courbure et de torsion jouent le même rôle que les coeffi. cients $c_{j k}^{i}$ définis dans $\S 7$. Soit $S$ la structure définie sur $H^{\prime}\left(V_{n}\right)$ par la forme différentielle qui à tout $\tilde{h}_{\infty} \varepsilon H^{\prime}\left(V_{n}\right)$ fait correspondre le corepère distingué d'origine $\tilde{h}_{x}$; le pseudogroupe des automorphismes locaux de cette structure $\mathfrak{S}$ est un pseudogroupe de LIE de type fini d'ordre et de degré égaux à 1 et le pseudogronpe des automorphismes locaux de la structure $s$ sur $V_{n}$ est un pseudogroupe de Lie de type fini, de degré 2.

Pour que la structure $s$ soit isotrope en tout point $x$ d' un voisinage $U$ d'un point de $V_{n}$ e'est-à-dire pour qu'il existe un automorphisme local $f$ de la structure $s$ (se prolongeant en un homéomorphisme $\bar{f}$ d'un ouvert de $H^{\prime}\left(V_{n}\right)$ sup un ouvert de $\left.H^{\prime}\left(V_{n}\right)\right)$ appliquant un repère arbitraire $\tilde{h}_{x} \varepsilon H^{\prime}\left(V_{n}\right)$ sur un repère arbitraire $\tilde{h}_{\infty}^{\prime} \varepsilon H^{\prime}\left(V_{n}\right)$, il faut et il suffit d'après l'étude faite dans $\& 7$, qu' en tout point $x \& U$, les composantes des tenseurs suivants soient indépendantes $d u$ repère $\tilde{h}_{\infty}$ et soient par conséquent des invariants pour la structure $s$ : tenseur de torsion, tenseur de courbure, tenseurs obtenus par différentiations successires de ces premiers tenseurs jusqu'à ce que tout invariant soit fonction des invariants obtenus précédem. ment. En particulier si les composantes des tenseurs de courbure et de torsion sont en chaque point $x \in U$ indépendantes du repère $\tilde{h}_{x}$ et sont des constap. tes, la structure $\mathfrak{z}$ est isotrope en tout point $x \varepsilon U$ et localement homogène dans $U$. La structure est localement équivalente à une structure dont le pseudogroupe des automorphismes locaux est déduit d'un groupe de Lie.

Dans le cas d'une structure non isotrope, on peut chercher à déterminer en chaque point $x \in V_{n}$ un corepère distingué sur $V_{n}$ ou une sous-famille distinguée de corepères. On applique les mêmes méthodes que dans A), en considérant, outre les composantes du teuseur de torsion, celles $d u$ tenseur de courbure. 
Le problème d'équivalence des structures riemanniennes (dont le groupe structural est le groupe orthogonal $O_{n}$ ) a été étudié par E. CARTAN [15] et S. CHERN [17].

\section{DEUXIÈE PARTIE}

Chapitre III.

\section{Structures de $R^{\text {nn }}$ subordonnées à sa structure vectorielle réelle.}

\section{Formes réelles d'un groupe linéaire complexe.}

Soit $\mathcal{S}$ un sons-groupe du groupe linéaire homogène $L^{\prime}{ }_{n}$ de l'espace vectoriel complexe $C^{n}$ e'est-à-dire du groupe de toutes les $n \times n$ matrices régulières à éléments complexes. Supposons que $\mathfrak{G}$ soit un groupe de LIE et que son algèbre de LIE soit un espace vectoriel complexe de dimension $p$ : $\mathfrak{G}$ est alors un groupe complexe; une forme réelle du groupe $\mathcal{G}$ est un sons-groupe de $\mathfrak{G}$ admettant comme algèbre de LIE un espace vectoriel réel de dimension $p$.

Soit dans $C^{n}$ une antiinvolutiou $z \rightarrow A(\bar{z})$ (où $z \varepsilon C^{n}$ ); cette antiinvolution est de première espèce si par un changement de coordonnées dans $C^{n}$, on peut la réduire à la forme canonique: $Z^{s} \rightarrow \bar{Z}^{s} \quad(s=1, \ldots, n)$. La transformée par l'antiinvolution de première espèce $A$ de la matrice $g \varepsilon L_{n}^{\prime}$ est : $g^{\prime}=A \bar{g} A^{-1}$ où $\bar{g}$ est la matrice dont les éléments sont les imaginaires conjugués de ceux correspondants de $g$; à l'antiinvolution $A$ dans $C^{n}$ correspond done dans l'espace des $n \times n$ matrices à éléments complexes l'antiinvolution $B: g \rightarrow A \tilde{g} A^{-1}$; cette antiinvolution $B$ est de première espèce car si $A$ est mise sous forme canonique, $B$ se réduit à : $g \rightarrow \bar{g}$. Comme l'algèbre de LIE de $L_{n}^{\prime}$ est isomorphe à l'espace de toutes les $n \times n$ matrices complexes, muni de la loi de composition: $[X Y]=Y X-X Y\left({ }^{6}\right)$, l'antiinvolution $B$ opère sur l'algèbre de LIE de $L_{n}^{\prime}$.

Supposons que le groupe $\mathcal{G}$ (qui est de dimension réelle $2 p$ ) soit invariant par une antiinvolution de première espèce $B$ et que le sous-groupe $G$ de $\mathfrak{G}$ engendré par les matrices $g \varepsilon \mathfrak{G}$ invariantes par $B$ soit de dimension $p$ : $G$ est alors une forme réelle de $\mathcal{G}$. En effet si par un changement de coordonnées, l'antiinvolution est mise sous forme canonique, toute matrice $g \& G$ est à éléments réels.

10. Structure vectorielle complexe [24].

Une structure vectorielle complexe dans l'espace numérique $R^{2 n}$, subor. donnée à sa structure d'espace vectoriel réel, est déterminée par un automorphisme $\mathfrak{g}$ de $R^{2 n}$ tel que $\mathfrak{g}^{2}=-1$. Un sous-espace $X_{p}$ (de dimension $p$ )

(6) Voir Chevalley [20]. 
de $R^{2 n}$ sera dit réel si $X^{p}$ et son transformé $\mathfrak{J} X_{p}$ par $\mathfrak{I}$ sont supplémentaires; un sous-espace $X_{q q}$, invariant par $\mathfrak{J}$, sera dit complexe (de dimension com. plexe q).

En prolongeant la transformation $\mathfrak{d}$ à l'espace vectoriel complexe $C^{2 n}$ dont $R^{2 n}$ est un sous-espace, on definit dans $C^{2 n}$ une transformation linéaire admettant les valeurs propres $\pm i$; les vecteurs propres correspondant a $-i$ et $+i$ engendrent respectivement deux sous-espaces supplémentaires imaginaires conjugues $\mathfrak{X}_{n}$ et $\overline{\mathfrak{X}}_{n}$ de dimension complexe $n$. Réciproquement tout sous-espace $\mathfrak{X}_{n}$ de $C^{\text {2n }}$ tel que $\mathfrak{X}_{n} \cap \overline{\mathscr{X}}_{n}=0$ détermine un automorphisme $\mathfrak{J}$ de carré -1 .

Soient $z^{1}, \ldots, z^{2 n}$ les coordonnées canoniques dans $C^{2 n}$ et soit $\mathfrak{J}_{0}$ l'automorphisme déterminé par les sous-espaces $\left(\mathscr{X}_{n}\right)_{0}$ et $\left(\overline{\mathscr{X}}_{n}\right)_{0}$ définis respectivement par les équations :

$$
z^{s^{\prime}}=0 ; \quad z^{s}=0 \quad\left(s=1, \ldots, n ; s^{\prime}=s+n\right) .
$$

Nous supposerons désormais (sauf mention contraire) que les indices latins varient de $1 \grave{a ̀} n$, les indices grecs de 1 à $2 n$ et que $s^{\prime}=\mathrm{s}+n$.

$R^{2 n}$ est défini par les equations: $z^{s^{\prime}}=z^{t}(s=1, \ldots, n)$ et si l'on pose: $z^{s}=x^{s}+i y^{s} ; z^{s}=x^{s}-i y^{s}$, les coordonnées $x^{s}$ et $y^{s}$ sont réelles dans $R^{2 n}$ : ce sont les coordonnées canoniques de $R^{2 n}$. La transformation $\mathfrak{J}_{0}$ est definie par: $z^{s} \rightarrow i z^{s}, z^{s \prime} \rightarrow-i z^{s t}$, ou encore par: $x^{s} \rightarrow-y^{s}, y^{s} \rightarrow x^{s}(s=1, \ldots, n)$.

La structure vectorielle complexe dans $R^{2 n}$ déterminée par l'automorphisme $\mathfrak{J}_{0}$ admet le groupe lineaire homogène complexe $L_{\text {" }}^{\prime}$ comme groupe d'antomorphismes. Ce groupe $L^{\prime}{ }_{n}$, considéré ccmme sous-groupe du groupe linéaire homogène $L_{2 n}$, est isomorphe à une forme réelle du groupe complexe $L_{n}^{\prime} \times L_{n}^{\prime}$ (sous-groupe de $L_{2 n}^{\prime}$ ): en effet soit $l_{2 n}$ le plus grand sous-groupe de $L_{2 n}^{\prime}$ laissant invariants les sous-espaces $\left(\mathscr{X}_{n}\right)_{0}$ et $\left(\overline{\mathscr{X}}_{n}\right)_{0} ; l_{2 n}$ qui peut etre identifié au groupe des matrices $\left(\begin{array}{cc}M & 0 \\ O & N\end{array}\right)$ (où $M$ et $N$ sont des $n \times n$ matrices régulières à éléments complexes) est isomorphe à $L_{n}^{\prime} \times L_{n}^{\prime}$; le groupe $L_{n}{ }^{\prime}$ est lo sous-groupe de $l_{2 n}$ engendré par les matrices $\left(\begin{array}{cc}M & \frac{0}{M} \\ 0 & \frac{1}{M}\end{array}\right)$ invariantes par l'antiinvolution $B$ de l'espace des $2 n \times 2 n$ matrices correspondant à l'antiinvolution $A$ de $C^{2 n}$ définie par: $z^{s \prime} \rightarrow \overline{z^{s}}, z^{s} \rightarrow \bar{z}^{s \prime}(s=1, \ldots, n)$.

\section{Structure de module unitaire paracomplexe sur $R^{2 n}$ [41].}

Soit $\sigma$ la structure déterminée dans l'espace vectoriel $R^{2 n}$ par la donnée de deux sous-espaces supplémentaires $X_{n}$ et $X^{\prime}{ }_{n}$ de même dimension $n$. Le couple $\left(X_{n}, X_{n}^{\prime}\right)$ définit un automorphisme involutif $\mathscr{F}$ de $R^{2 n}$ (c'est-à-dire tel que $\mathcal{g}^{2}=1$ ), dont les vecteurs propres correspondant aux valeurs propres +1 et -1 engendrent respectivement les sous-espaces $X_{n}$ et $X^{\prime}{ }_{n}$ (appelés respectivement sous-espace positif et sous-espace négatif de $\mathscr{f}$ [21]). Inverse. ment une structure $\sigma$ dans $R^{2 n}$ est déterminée par la donnée d'une 
$(n, n)$-involution $\mathfrak{F}$ de $R^{2 n}$ [en désignant par $(p, 2 n-p)$-involution un automorphisme involutif de $R^{i n}$ dont les sous-espaces positif et négatif sont de dimensions respectives $p$ et $2 n-p$ ].

Soit $e$ un vecteur de $R^{2 n}$ non situé dans $X_{n}$ ou $X^{\prime}{ }_{n}$; les vecteurs $e$ et $\mathfrak{F} e$ déterminent un plan invariant par $\mathcal{F}$, coupant suivant uue droite chacun des sous-espaces $X_{n}$ et $X_{n}^{\prime}$ car: $e+\mathscr{F} e \varepsilon X_{n}, e-e \mathfrak{F} \varepsilon X_{n}^{\prime}$. Les transformations linéaires $\frac{1+\mathfrak{F}}{2}$ et $\frac{1-\mathfrak{F}}{2}$ telles que: $\left(\frac{1+\mathfrak{F}}{2}\right)^{2}=\frac{1+\mathfrak{F}}{2},\left(\frac{1-\mathfrak{F}}{2}\right)^{2}=$ $=\frac{1-\mathfrak{g}}{2}$ et $\left.\left(\frac{1+\mathfrak{F}}{2}\right)\left(\frac{1-\mathfrak{F}}{2}\right)=0\right]$ qui à tout vecteur $e$ font correspondre respectivement les vecteurs $\frac{e+\mathscr{F} e}{2}$ et $\frac{e-\mathscr{f} e}{2}$ sont des projections sur $X_{n}$ et $X^{\prime}{ }_{n}$ et non pas des automorphismes de $R^{2 n}$.

Soit $\left(e_{1}, \ldots, e_{n}\right)$ un ensemble de $n$ vecteurs linéairement indépendants non situés dans $X_{n}$ ou $X^{\prime}{ }_{n}$ et dont les projections sur $X_{n}$ sont linéairement indépendantes: les vecteurs $e_{1}, \mathfrak{F} e_{1}, \ldots, e_{n}$, $\mathfrak{F} e_{n}$ constituent une base de $R^{2 n}$. Considérons maintenant la $(n, n)$-involution $\mathfrak{F}_{0}$ définie par: $x^{s} \rightarrow y^{s}, y^{s} \rightarrow x^{s}$ $(s=1, \ldots, n)$. Le sous-espace positif $\left(X_{n}\right)_{0}$ et le sous-espace négatif $\left(X^{\prime}{ }_{n}\right)_{0}$ de $\mathfrak{F}_{0}$ sont définis respectivement par les équations :

$$
x^{s}-y^{s}=0 ; x^{s}+y^{s}=0 \quad(s=1, \ldots, n),
$$

où $x^{1}, \ldots, x^{n}, y^{1}, \ldots, y^{n}$ sont les coordonnées canoniques dans $R^{2 n}$. A ces coordonnées correspond la base canonique de $R^{2 n}:\left(\varepsilon_{1}, \varepsilon_{n+1}, \ldots, \varepsilon_{n}, \varepsilon_{2 n}\right)$ et l'on a: $\varepsilon_{n+s}=\mathfrak{F}_{0} \varepsilon_{s}(s=1, \ldots, n)$. A cette base est associée également un système canonique de $n$ coordonnées «paracomplexes» $\stackrel{\circ}{*}^{s}=x^{s}+i y^{s} \quad(s=1$, $\ldots, n)$ en désignant par nombre paracomplexe tout élément d' une extension quadratique [5] du corps des réels, de base $(1, j)$ avec $j^{2}=1$. L'algèbre $A$ des nombres paracomplexes ne forme pas un corps; en effet soit $\stackrel{\bar{z}}{z}=x-i y$ le conjugué du nombre paracomplexe $\ddot{z}=x+i y$; la norme de $z$ est: $N(z)=\ddot{z} \ddot{z}=(x+j y)(x-i y)=x^{2}-y^{2}$ et $N(z)$ n' est pas définie positive. $L a$ structure $\sigma_{0}$ de $R^{2 n}$ déterminée par la $(n, n)$-involution $\mathfrak{F}_{0}$ est donc une structure de module unitaire $A^{n}$ par rapport $\grave{a}$ l'algèbre des nombres paracomplexes. Le groupe des automorphismes de cette structure, que nous désignerons par $\stackrel{\circ}{L}_{n}^{\prime}$ sera appelé groupe linéaire homogène paracomplexe. Ce groupe peut être identifié au groupe des $n \times n$ matrices régulières à eléments paracomplexes (pour qu'une matrice à éléments paracomplexes soit régulière c'est-à-dire admette une inverse il ne suffit pas que son déterminant soit nul, l'algèbre $A$ ne formant pas un corps). Le groupe $L^{\prime}{ }_{n}$ laissant invariants $\left(X_{n}\right)_{0}$ et $\left(X^{\prime}{ }_{n}\right)_{0}$ est isomorphe à $L_{n} \times L_{n} ; \stackrel{\circ}{L}_{n}^{\prime}$ est done isomorphe à la forme réelle de $L_{n}^{\prime} \times L_{n}^{\prime}$ correspondant à l'antiinvolution de $C^{2 n}: z^{s^{\prime}} \rightarrow \bar{z}^{s^{\prime}}, z^{s} \rightarrow \bar{z}^{s}(s=1, \ldots, n)$.

On désignera par droites paracomplexes le sous-variétés linéaires de $A^{n}$ à une dimension paracomplexe, e' est-à-dire les plans de $R^{2 n}$ invariants 
par $\mathfrak{F}_{0}$ et non situés dans $\left(X_{n}\right)_{0}$ ou $\left(X_{n}^{\prime}\right)_{0}$. La variété $P_{n-1}(A)$ des droites paracomplexes de $A^{n}$ peut être identifiée à la variété des couples de droites $\left(d, d^{\prime}\right)$ où $d \varepsilon\left(X_{n}\right)_{0}, d^{\prime} \varepsilon\left(X_{n}^{\prime}\right)_{0}$. La variété $P_{n-1}(A)$ que nous appellerons espace projectif paracomplexe a $(n-1)$ dimensions peat donc etre identifiée au produit topologique $P_{n-1}(R) \times P_{n-1}(R), P_{n-1}(R)$ désignant l'espace projectif réel à $(n-1)$ dimensions. Alors que la donnée de l'automorphisme $\mathscr{F}_{0}$ tel que $\left(\mathscr{F}_{0}\right)^{2}=-1$ détermine une orientation de $R^{2 n}$, il n'en est pas de même pour l'involution $\mathfrak{F}_{0}$.

12. Antomorphismes de carré -1 et involutions échangeables.

a) Soient dans $R^{i n}$ deux involutions $\mathscr{F}$ et $\mathscr{F}$ échangeables, e'est-à-dire telles que: $\mathfrak{d} \mathscr{F}=\mathfrak{F} \mathfrak{I}$. $L$ involution $\mathfrak{H}=\mathfrak{J} \mathscr{F}$ est aussi échangeable avec $\mathfrak{J}$ et $\mathfrak{F}$. Soient $X$ et $X^{\prime}$ les sous-espaces positif et négatif de $\mathfrak{J}, Y$ et $Y^{\prime}$ ceux de $\mathfrak{F}$; $Z$ et $Z^{\prime}$ ceux de $\mathfrak{K}$; le sous-espace $X$ est invariant par $\mathscr{F}$ et $\mathfrak{H}$, donc $X$ est la réanion des deux sous-espaces $X_{1}$ et $X_{z}, X_{1}$ étant l'intersection $Y \cap Z$ et $X_{2}=Y^{\prime} \cap Z^{\prime}$. On démontre de même que $X^{\prime}$ est la réunion de $X_{3}=Y \cap Z^{\prime}$ et $X_{4}=Y^{\prime} \cap Z$. Par suite les sous-espaces $Y, Y^{\prime}, Z, Z^{\prime}$ sont respectivement engendrés par la réunion de: $X_{1}$ et $X_{3}, X_{2}$ et $X_{4}, X_{1}$ et $X_{4}, X_{2}$ et $X_{3}$; soient $p_{1}, p_{2}, p_{3}, p_{4}$ les dimensions des sous-espaces $X_{1}, X_{2}, X_{3}, X_{4}$ (on a: $p_{1}+p_{z}+p_{3}+p_{4}=2 n$ ). Le groupe des automorphismes de la structure définie sur $R^{2 n}$ par la donnée des involutions $\mathfrak{I}$ et $\mathfrak{F}$ laisse invariant chacun des sous-espaces $X_{1}, X_{2}, X_{3}, X_{4}$ : ce groupe est isomorphe a $L_{p_{1}} \times L_{p_{2}} \times L_{p_{3}} \times L_{p_{4}}$. En particulier si les involutions $\mathfrak{J}, \mathfrak{F}, \mathfrak{K}$ sont des $(n, n)$-involutions, on doit avoir: $p_{1}=p_{2}=p_{3}=p_{4}$; donc dans $c \theta$ cas $n=2 p$ et le groupe des automorphismes est isomorphe à $L_{p} \times L_{p} \times L_{p} \times L_{p}$.

A l'énsemble de quatre sous-espaces deux à deux supplémentaires $X_{1}$, $X_{2}, X_{3}, X_{4}$ dont la réunion est $R^{2 n}$, correspondent quatre ensembles de trois involutions deux à deux échangeables (on prend successivement comme intersection des sous-espaces positifs de trois involutions chacun des sousespaces $X_{1}, X_{2}, X_{3}, X_{4}$ ).

b) Soient dans $R^{2 n}$ deux automorphismes $\mathfrak{J}$ et $\mathscr{f}$ tels que: $\mathfrak{F}^{2}=-1$, $\mathfrak{F}^{2}=-1, \mathfrak{J F}=\mathfrak{F} \mathfrak{g}$. L'automorphisme $\mathfrak{K}=\mathfrak{g} \mathscr{F}$ est une involution ot l'on a : $\mathfrak{d} \mathfrak{K}=\mathfrak{T} \mathfrak{J}=-\mathfrak{F}, \mathfrak{F} \mathfrak{K}=\mathfrak{K} \mathfrak{F}=-\mathfrak{J}$. Inversement la donnée de l'automorphisme $\mathfrak{J}$ et de l'involution $\mathfrak{H}$ tels que $\mathfrak{J} \mathfrak{H}=\mathfrak{K} \mathfrak{J}$ détermine l'automorphisme $\mathfrak{F}=\mathfrak{d} \mathfrak{d}$. Les sous-espaces positif et négatif $X$ et $X^{\prime}$ de $\mathfrak{H}$ sont invariants par $\mathfrak{J}$ et $\mathscr{F}$ : $X$ et $X^{\prime}$ sont de dimension paire $q$ et $2 n-q$; done $n=2 p$.

La structure definie sur $R^{4 p}$ par la donnée de $\mathfrak{J}$ et $\mathfrak{F}$ peut également être déterminée par la donnée de l'automorphisme $\mathfrak{g}$ (d'où une structure d'espace vectoriel complexe dans $R^{i n}$ ) et de deux sous-espaces complexes (cf. $\S 10$ ) supplémentaires $X$ et $X^{\prime}$. Cette structure admet comme groupe d'automorphismes un groupe isomorphe au groupe $L_{q}^{\prime} \times L_{2 p-q}^{\prime}$. En particulier si $\mathfrak{H}$ est une $(2 p, 2 p)$-involution le groupe des automorphismes est isomorphe à $L_{p}^{\prime} \times L_{p}^{\prime}$. 
13. Structure d'espace vectoriel quaternionien [24] et de module unitaire quaternionien de deuxième espèce [39].

a) Soient dans $R^{2 n}$ deux automorphismes $\mathfrak{J}$ et $\mathfrak{g}$, de carré -1 , tels que: $\mathfrak{g} \mathfrak{F}=-\mathfrak{g} \mathfrak{J}=\mathfrak{K} ;$ on a: $\mathfrak{J K}^{2}=-1$ ef $\mathfrak{d} \mathfrak{K}=-\mathfrak{H} \mathfrak{J}=\mathfrak{F}$. Aux automorphismes $\mathfrak{J}, \mathfrak{F}, \mathfrak{H}$ est associé le corps des quaternions usuels de base $(1, i, j, k)$ telle que:

$$
i^{2}=-1, j^{2}=-1, k^{2}=-1, i j=-j i=k, k i=-i k=j, j k=-k j_{1}=i .
$$

Donc la donnée dans $R^{2 n}$ d'une structure d'espace vectoriel complexe (déterminée par un antomorphisme $\mathfrak{J}$ tel que $\mathfrak{J}^{2}=-1$ ) et $\mathrm{d}^{\prime}$ un automorphisme $\mathfrak{F}$ de $R^{2 n}$ tel que: $\mathfrak{F}^{2}=-1$ et $\mathfrak{g} \mathscr{F}=-\mathfrak{g} \mathfrak{J}$ détermine dans $R^{2 n}$ une structure d'espace vectoriel quaternionien, admettant un groupe d'automorphismes isomorphe au groupe linéaire quaternionien $L_{p}^{\prime \prime}[24]$.

b) La donnée dans $R^{2 n}$, ( $n$ étant quelconque) d'une structure d'espace vectoriel complexe (on d'un antomorphisme $\mathfrak{g}$ de carré -1 ) et $d$ 'un sousespace réel (of. $\$ 10) X_{n}$ de dimension $n$ déterminè également dans $R^{z n}$ une structure de module unitaire paracomplexe: le sous-espace $X_{n}$ et son transformé $\mathfrak{J} X_{n}$ étant supplémentaires, le couple $\left(X, X_{n}^{\prime}\right)$ détermine une $(n, n)$ involution $\mathfrak{F}$.

Soit $v$ un vecteur de $R^{2 n}$. On a: $v=e+e^{\prime}$, où $e \varepsilon X_{n}, e^{\prime} \varepsilon X_{n}^{\prime} ;$ d'où $\mathfrak{J} v=\mathfrak{J} e+\mathfrak{d} e^{\prime}=e-e^{\prime} ; \mathfrak{d} \mathfrak{F} v=\mathfrak{d} e-\mathfrak{J} e^{\prime} ; \mathfrak{d} v=\mathfrak{d} e+\mathfrak{J} e^{\prime}$ avec: $\mathfrak{I} e \equiv X_{n}^{\prime}, \mathfrak{d} e^{\prime} \varepsilon X_{n} ;$ done $\mathfrak{F} \mathfrak{J} v=\mathfrak{d} e^{\prime}-\mathfrak{J} e$ et par suite: $\mathfrak{d} \mathscr{F}=-\mathfrak{F} \mathfrak{I}$. Réciproquement soient $\mathfrak{J}$ et $\mathfrak{F}$ deux automorphismes de $R^{2 n}$ tels que: $\mathfrak{I}^{\mathfrak{}}=-1, \mathfrak{F}^{\mathfrak{2}}=1$, $\mathfrak{g} \mathscr{F}=-\mathfrak{g} \mathfrak{g}$. Tout vecteur propre de $\mathfrak{F}$ correspondant à la valeur propre +1 est transformé par $\mathfrak{J}$ en un vecteur propre correspondant à $-1: \mathscr{F}$ est dono une $(n, n)$-involution.

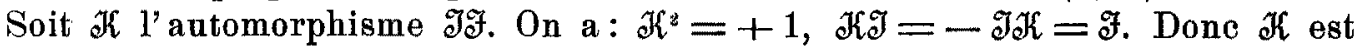
une $(n, n)$-involution dont les sous-espaces positif et négatif sont échangés par $\mathfrak{J}$. La donnée du couple $(\mathfrak{d}, \mathfrak{T})$ détermine $\mathfrak{F}$. De même la donnée du couple $(\mathfrak{F}, \mathfrak{R})$ d'involutions de $R^{: n}$ telles que $\mathscr{F} \mathfrak{K}=-\mathfrak{o} \mathscr{H} F$ détermine l'automorphisme $\mathfrak{J}=\mathfrak{H} \mathscr{F}$ tel que $\mathfrak{J}^{2}=-1 ; \mathfrak{J}$ échange le sous-espaces positif et négatif de $\mathscr{F}$ et de $\mathfrak{T}$ qui sont donc des $(n, n)$-involutions ; d' où :

THEORÈME 13.1. - Soient $\mathscr{F}$ et $\mathfrak{H}$ deux automorphismes de l'espace vectoriel $R^{2 n}$ tels que $\mathfrak{F}^{2}=1, \mathfrak{H}^{2}=1$, $\mathfrak{F} \mathfrak{K}=-\mathfrak{H} \mathfrak{F}$. Chacun de ces automorphismes est une $(n, n)$-involution dont les sous-espaces positif et négatif sont échangés par l'autre involution ainsi que par l'automorphisme $\mathfrak{J}=\mathfrak{H} \mathfrak{F}$, lequel définit dans $R^{2 n}$ une structure d'espace vectoriel complexe.

THÉOR亡Me 13.2 - Tout triplet $\left(X_{n}, X^{\prime}{ }_{n}, Y_{n}\right)$ de sous-espaces de $R^{2 n}$, de dimension $n$, deux à deux supplémentaires détermine un triplet $(\mathfrak{J}, \mathfrak{F}, \mathfrak{K})$ d'automorphismes de $R^{2 n}$ tels que : $\mathfrak{J}^{2}=-1, \mathfrak{G}^{2}=\mathfrak{I K}^{2}=1$, $\mathfrak{I} \mathfrak{F}=-\mathfrak{F} \mathfrak{J}=\mathfrak{T}$, $X_{n}$ et $X_{n}^{\prime}$ étant les sous-espaces positif et négatif de $\mathfrak{F}, Y_{n}$ le sous-espace positif de $\mathfrak{H}$.

DÉmonstration: le couple $\left(X_{n}, X^{\prime}{ }_{n}\right)$ détermine une $(n, n)$-involution $\mathfrak{F}$ qui transforme tout vecteur e $\varepsilon \dot{Y}_{n}$ en un vecteur $\mathscr{F} e$ non situé dans $Y_{n}$; en 
effet le vecteurs $e$ et $\mathfrak{J} e$, qui sont distincts, engendrent un plan $P$ coupant $X_{n}$ et $X_{n}^{\prime}$; le plan $P$ (et par suite $\mathscr{F} e$ ) n'est donc pas contenu dans $Y_{n}$; le transformé $\mathfrak{F} Y_{n}$ du sous-espace $Y_{n}$ par l'automorphisme $\mathscr{F}$ est par conséquent na sous-espace $Y_{n}^{\prime}$ tel que les quatre sous-espaces $X_{n}, X_{n}^{\prime}, Y_{n}$ et $Y_{n}^{\prime}$ soient deux à deux supplémentaires. Soit $\mathscr{K}$ l'involution associée au couple $\left(Y_{n}, Y_{n}^{\prime}\right)$ et soit $v=e+e^{\prime}$ (où $e \varepsilon Y_{n}, e^{\prime} \varepsilon Y_{n}$ ) un vecteur quelconque de $R^{2 n}$. On a alors : $\mathfrak{A} v=\mathfrak{H} e+\mathfrak{H} e^{\prime}=e-e^{\prime} ; \mathfrak{F} \mathfrak{H} v=\mathfrak{F} e-\mathfrak{F} e^{\prime} ; \mathfrak{d} \mathfrak{K} \mathfrak{F} v=\mathfrak{H} \mathscr{F} e+\mathfrak{H} \mathfrak{F} e^{\prime}=$ $=-\mathfrak{f} e+\mathfrak{J} e^{\prime}\left(\operatorname{car} \mathfrak{F} e \varepsilon Y_{n}^{\prime}\right.$ et $\mathfrak{J} e^{\prime} \varepsilon Y_{n}$. Donc $\mathscr{F} \mathfrak{H}=-\mathfrak{H} \mathscr{F}=-\mathfrak{J}$ et $\mathfrak{J}^{\mathfrak{z}}=-1$.

Considérons la structure $s_{0}$ de $R^{2 n}$ déterminée par la donnée de l'automor* phisme $\mathfrak{J}_{0}$ défini dans $\S 10$ et de l'involution $\mathfrak{F}_{0}$ délinie dans $\$ 11$ c'est-à-dire par les transformations $\mathfrak{I}_{0}\left(x^{s} \rightarrow-y^{s}, y^{s} \rightarrow x^{s}\right)$ et $\mathfrak{F}_{0}\left(x^{s} \rightarrow y^{s}, y^{s} \rightarrow x^{s}\right)$. On a $\mathfrak{J}_{0} \mathfrak{F}_{0}=-\mathfrak{F}_{0} \mathfrak{J}_{0}=\mathfrak{K}_{0}$ et l'involution $\mathfrak{K}_{0}$ est definie par: $x^{s} \rightarrow x^{s}, y^{s} \rightarrow-y^{s}$. Soient $\left(X_{n}\right)_{0}$ et $\left(X^{\prime}{ }_{n}\right)_{0}$ les sous-espaces positif et négatif de $\mathfrak{F}_{0},\left(Y_{n}\right)_{0}$ et $\left(Y^{\prime}{ }_{n}\right)_{0}$ ceux de $\mathfrak{K}_{0} ;\left(X_{n}\right)_{\theta},\left(X_{n}^{\prime}\right)_{0},\left(Y_{n}\right)_{0},\left(\bar{Y}_{n}^{\prime}\right)$ sont définis respectivement par les equations :

$$
x^{s}-y^{s}=0, \quad x^{s}+y^{s}=0, \quad y^{s}=0, \quad x^{s}=0, \quad(s=1, \ldots, n) .
$$

Le groupe des automorphismes de $s_{3}$ (que nous désignerons par $\widehat{L_{n}}$ ) laisse invariant chacun des sous-espaces $\left(X_{n}\right)_{0},\left(X_{n}^{\prime}\right)_{0},\left(Y_{n}\right)_{0},\left(Y_{n}^{\prime}\right): \widehat{L_{n}}$ est isomorphe au groupe linéaire homogène réel $L_{n}$. Oe groupe $\widehat{L}_{n}$ laisse également invariant tout sous-espace $X_{n}{ }^{(\mu)}$ defini par les equations:

$$
x^{s}+\mu y^{s}=0 \quad(s=1, \ldots, n),
$$

où $\mu$ est un paramètre réel. L'ensemble $F$ des sous-espaces $X_{n}\langle\mu\rangle$ sera appelé faisceau de sous-espaces: les sous-espaces de ce faisceau sont en correspondance biunivoque avec les points de la droite réelle. Au triplet $\left(\mu_{1}, \mu_{2}, \mu_{3}\right)$ de points de cette droite, correspond le triplet $\left(X_{n}^{\left(\mu_{1}\right)}, X_{n}{ }_{n}^{\left(\mu_{2}\right)}, X_{n}\left(\mu_{3}\right)\right)$ de sousespaces d'où le triplet $\left(\mathfrak{J}_{0}, \mathfrak{F}_{\cap}, \mathfrak{J}_{0}\right)_{\mu_{1} \mu_{\mu} \mu_{s}}$ d'automorphismes. Le sous-espace négatif de $\left(\mathfrak{R}_{0}\right)_{\mu_{1} \mu_{2} \mu_{3}}$ correspond au conjugué harmonique de $\mu_{3}$ par rapport à $\mu_{1}$ et $\mu_{z}$. Aux automorphismes $\mathfrak{J}_{0}, \mathfrak{F}_{0}, \mathfrak{H}_{0}$ est associée une algèbre quaternionienne de deuxième espèce $\mathfrak{A}$ sur le corps des réels $\left({ }^{7}\right)$, de base $(1, i, j, k)$ avec:

$$
i^{2}=-1, j^{2}=1, k^{2}=1, i j=-j i=k, j k=-k j=-i, k i=-i k=j .
$$

Soit un quaternion $q=a_{0}+a_{1} i+a_{2} j+a_{3} k$, son conjugué $\bar{q}=a_{0}-a_{1} i-$ $-a_{2} j-a_{3} k$ et la norme de $q$ est: $N(q)=q \bar{q}=\left(a_{0}\right)^{2}+\left(a_{1}\right)^{2}-\left(a_{2}\right)^{2}-\left(a_{3}\right)^{2}$. L'algèbre $\mathfrak{Q}$ n'est donc pas un corps. Les bases $\left(1, i^{\prime}, j^{\prime}, k^{\prime}\right)$ de l'algèbre qui vérifient les relations (13.3) sont en correspondance biunivoque avec les triplets $\left(X_{n}^{\left(\mu_{3}\right)}, X_{n}{ }^{\left.j \mu_{2}\right)}, X_{n}{ }^{\left(\mu_{3}\right)}\right) ;$ on en déduit que le groupe $\mathfrak{L}$ des automorphismes de l'algèbre $\mathfrak{Q}$ est isomorphe au groupe des transformations homographiques. Ce groupe $\mathcal{L}$ opère d' une manière simplement transitive sur la quadrique $Q_{3}$

(7) On réservera le terme quaternionien pour les quaternions usuels [b]. 
d'équation: $\left(X^{1}\right)^{2}+\left(X^{2}\right)^{2}-\left(X^{3}\right)^{2}-\left(X^{4}\right)^{2}=1$, dont tout point peut etre représenté par un quaternion de norme 1.

Supposons $n=2 p$. La donnée des automorphismes $\mathfrak{J}_{0}, \mathscr{F}_{0}, \mathfrak{X}_{0}$ détermine dans $R^{s p}$ une structure de module unitaire $\mathfrak{K}^{p}$ sur l'algèbre $\mathfrak{G}$.

L'automorphisme $\mathscr{F}_{0}$ permet d'identifier $R^{4 p}$ à l'espace vectoriel complexe $C^{2 p}$; soient $z^{1}, \ldots, z^{2 p}$ les coordonnées canoniques dans $C^{2 p}$. Si l'on pose: $Z^{s}=z^{s}-i z^{\prime \prime}, Z^{s^{\prime}}=z^{\prime \prime}-i z^{s}\left(s=1, \ldots, p ; s^{\prime}=s+p\right)$, la transformation $\mathfrak{J}_{0}$ est définie par:

$$
Z^{s} \rightarrow \bar{Z}^{s,}, \quad Z^{s \prime} \rightarrow \bar{Z}^{s} \quad\left(s=1, \ldots, p ; s^{\prime}=s+p\right)
$$

On peut alors définir dans $\mathfrak{T R}^{p}$ les coordonnées canoniques: $q^{*}=Z^{*}+j Z^{\prime \prime}=$ $=u^{s}+i v^{s}+j u^{s \prime}+k v^{s \prime}$ où $u^{s}+i v^{s}=Z^{s}, u^{s \prime}+i v^{s \prime}=Z^{s \prime}$ e' est-à-dire $u^{s}=$ $=x^{s}+y^{s t}, v^{s}=y^{s}-x^{s^{\prime}}, u^{s^{\prime}}=x^{s^{\prime}}+y^{s}, v^{s \prime}=y^{s^{\prime}}-x^{s}$. Le sous-groupe $i^{\prime \prime}{ }_{p}$ du groupe $L_{2 p}^{\prime}$ qui laisse invariante la transformation $\mathscr{F}_{0}$ sera désigné par par groupe linéaire quaternionien de deuxième espèce : ${ }^{\circ}{ }^{\prime \prime}{ }_{p}$ est identique à $\widehat{L}_{2 p}$. Le sous-groupe $\mathfrak{L}_{p}$ de $L_{p}^{\prime \prime}$ qui laisse invariante la forme $\Sigma q^{s} q^{*}=\Sigma\left[\left(u^{v}\right)^{2}+\right.$ $\left.+\left(v^{s}\right)^{2}-\left(u^{s}\right)^{2}-\left(v^{s^{\prime}}\right)^{2}\right]=4 \Sigma\left(x^{s} y^{s}-x^{s^{\prime}} y^{s}\right)$ sera appele groupe unilaire quaternionien de deuxième espèce; ce groupe est isomorphe au groupe symplectique réel $\tilde{L}_{2 p}$. Le groupe $\mathcal{L}_{1}$ est identique à $\mathscr{L}$.

14. Structures admettant le groupe unitaire [24] et le groupe «paraunitaire » comme groupe d'automorphismes [41].

a) A la donnée d'une forme quadratique definie positive $F[(d e$ rang $2 n$ ) et d'un automorphisme $\mathfrak{d}$ de $R^{2 n}$, de carré -1 , laissant invariante $F$, sont associées une forme quadratique exterieure $Q$ de rang $2 n$, échangeable avec $F$ et une forme d'Hermite définie positive $\Phi$ [24]; en effet on pent trouver dans $R^{2 n}$ un système de coordonnées $\alpha^{1}, \ldots, \alpha^{n}, \beta^{1}, \ldots, \beta^{n}$ tel que la transformation $\mathfrak{d}$ étant définie par: $\alpha^{s} \rightarrow-\beta^{s}, \beta^{s} \rightarrow \alpha^{s}(s=1, \ldots, n)$, la forme 1 s'ecrive :

$$
F=\left(\alpha^{1}\right)^{2}+\ldots+\left(\alpha^{n}\right)^{2}+\left(\beta^{1}\right)^{2}+\ldots+\left(\beta^{n}\right)^{2} ;
$$

le groupe des automorphismes de la structure définie par la donnée de $F$ et $\mathfrak{d}$ laisse invariantes les formes :

$$
\left.\mathrm{Q}=i \mid t^{1} \wedge \bar{t}^{1}+\ldots+t^{n} \wedge \bar{t}^{n}\right), \quad \Phi=t^{1} \bar{t}^{1}+\ldots+t^{n} \bar{t}^{n}
$$

où $t^{s}=\alpha^{s}+i \beta^{s}$. La donnée de $\Omega$ et de $F$ détermine dans $R^{2 n}$ un automor. phisme $\mathfrak{J}$ de carré -1 (produit des dualités par rapport à $\Omega$ et $F$ ), d'où une structure d'espace vectorial complexe. Par contre à la donnée de la forme $\Omega$ et $d^{\prime}$ un automorphisme $\mathfrak{J}$ de carré -1 , sont associées une forme quadratique et une forme d'Hermime qui ne sont pas nécessairement définies positives; en effet on peut trouver dans $R^{2 n}$ un système de coordonnées $\lambda^{4}, \ldots, \lambda^{n}, \mu^{1}, \ldots, \mu^{n}$ (d'où les coordonnées complexes $\tau^{s}=\lambda^{*}+i \mu^{*}$ ) tel que $\mathfrak{J}$ 
étant définie par: $\tau^{s} \rightarrow i \tau^{s}, \bar{\tau}^{s} \rightarrow-\overline{i \tau} s(s=1, \ldots, n), \Omega$ puisse $s^{\prime}$ écrire :

$$
Q=i\left(\tau^{1} \wedge \bar{\tau}^{1}+\ldots+\tau^{k} \wedge \bar{\tau}^{k}-\tau^{k+1} \wedge \bar{\tau}^{k+1} \ldots \tau^{n} \wedge \tau^{n}\right)
$$

à cette structure sont associées la forme d' HanMITE

$$
\Phi=\bar{\tau} \tau^{1}+\ldots+\tau^{\bar{k}} \tau^{k}-\tau^{k+1} \bar{\tau} \tau^{k+1}-\ldots-\tau^{\bar{n}} \tau^{n},
$$

et la forme quadratique

$$
F^{(k)}=\left(\lambda^{1}\right)^{2}+\left(\mu^{1}\right)^{2}+\ldots+\left(\lambda^{k}\right)^{2}+\left(\mu^{k}\right)^{2}-\left(\lambda^{k+1}\right)^{2}-\left(\mu^{k+1}\right)^{2}-\ldots-\left(\lambda^{n}\right)^{2}-\left(\mu^{n}\right)^{2} .
$$

En particulier la transformation $\mathfrak{J}_{0}\left(x^{s} \rightarrow-y^{s}, y^{s} \rightarrow x^{s}\right)$ où les $x^{s}$ et $y^{s}$ sont les coordonnées canoniques dans $R^{2 n}$ laisse invariante la forme

$$
F_{0}=\left(x^{1}\right)^{2}+\left(y^{1}\right)^{2}+\ldots+\left(x^{n}\right)^{2}+\left(y^{n}\right)^{2} .
$$

Le sous-groupe de $L_{n}^{\prime}$ laissant invariante $F_{n}$ est le groupe unitaire $U_{n}$; ce groupe laisse invariantes les formes:

$$
\mathrm{\Omega}_{0}=i\left(z^{1} \wedge \overline{z^{1}}+\ldots+z^{n} \wedge \overline{z^{n}}\right), \quad \Phi_{0}=z^{1} z^{1}+\ldots+z^{n} z^{n}
$$

où les $z^{s}$ sont les coordonnees canoniques dans $C^{n} ; U_{n}$ laissant invariante la forme $\Omega_{0}$ qui peut -s'écrire: $2\left(x^{1} \wedge y^{1}+\ldots+x^{n} \wedge y^{n}\right)$ est un sous-groupe du groupe symplectique réel $\tilde{L_{2 n}}$.

De même nous désignerons par groupe unitaire d'espèce $k$ le sous-groupe $U_{n}\langle k\rangle$ de $L_{n}^{\prime}$ laissant invariante la forme

$$
F_{0}{ }^{\langle k\rangle}=\left(x^{1}\right)^{2}+\left(y^{1}\right)^{2}+\ldots+\left(x^{k}\right)^{2}+\left(y^{k}\right)^{2}-\left(x^{k+1}\right)^{2}-\left(y^{k+1}\right)^{2}-\ldots-\left(x^{n}\right)^{2}-\left(y^{n}\right)^{2} .
$$

b) A la donnée d'une forme quadratique extérieure $\Omega$ de rang $2 n$ et d'une $(n, n)$-involution $\mathscr{F}$ dont les sous-espaces positif et négatif $X_{n}$ et $X_{n}^{\prime}$ sont intégraux de $\boldsymbol{\Omega}$ (c'est-à-dire sont tels que la restriction de $\Omega$ a $X_{n}$ ou $X^{\prime}{ }_{n}$ soit nulle), est associée une forme quadratique $F^{\prime}$ décomposable en une somme de $n$ carrés positifs et de $n$ carrés négatifs: $X_{n}$ et $X_{n}^{\prime}$ sont intégraux de cette forme $F^{\prime}$. Alors que la donnée de $\Omega$ et $F$ définit un seul automorphisme $\mathfrak{I}$, aux formes $\Omega$ et $F^{\prime}$ qui ont en commun $2^{n-1}$ couples $\left(X_{n}, X_{n}^{\prime}\right)$ de sous-espaces supplémentaires intégraux, sont associés $2^{n-1}(n, n)$-involutions $\mathfrak{F}$.

En particulier les sous-espaces positif et négatif $\left(X_{n}\right)_{0}$ et $\left(X_{n}^{\prime}\right)_{0}$ de l'invo. lution $\mathfrak{g}_{0}\left(x^{s} \rightarrow y^{s}, y_{s} \rightarrow x^{s}\right)$ sont intégraux de la forme $\Omega_{0}$ définie dans $\left.a\right)$ et qui pent s' écrire: $\Omega_{0}=\Sigma\left(x^{s}+y^{s}\right) \wedge\left(x^{s}-y^{s}\right)$. Le sous-groupe $\stackrel{\circ}{U}_{n}$ de $\stackrel{\circ}{L}_{n}^{\prime}$ qui laisse invariante la forme $\Omega_{0}$ sera appele groupe para-unitaire. Ce groupe $\dot{U}_{n}$ laisse invariante la forme quadratique

$$
F^{\prime}{ }_{0}=\left(x^{1}+y^{1}\right)\left(x^{1}-y^{1}\right)+\ldots+\left(x^{n}+y^{n}\right)\left(x^{n}-y^{n}\right) .
$$

En introduisant les coordonnées paracomplexes $\dot{z}^{s}=x^{s}+i y^{s}$, on peut écrire $F^{\prime}{ }_{0}$ sous la forme:

$$
\Phi_{0}^{\prime}=\stackrel{i}{z^{1}} \bar{z}^{i}+\ldots+\stackrel{\circ}{z}^{n} \mathscr{z}^{n}
$$

La forme $\Phi^{\prime}$, sera dite parahermitienne. 
c) Soit $\tilde{l}_{2 n}$ le sous-groupe de $L_{2 n}^{\prime}$ laissant invariants les sous-espaces supplémentaires $\left(\mathscr{X}_{n}\right)_{0}$ et $\left(\hat{\mathscr{X}}_{n}\right)_{0}$ de $C^{2 n}$ ainsi que la forme quadratique extérieure complexe $\Sigma z^{*} \wedge z^{s}$ dont $\left(\mathscr{X}_{n}\right)_{0}$ et $\left(\overline{\mathscr{X}}_{n}\right)_{0}$ sont intégraux; $\tilde{l}_{2 n}$ peut etre identifié au groupe des matrices $\left(\begin{array}{cc}M & 0 \\ O & M^{\prime}\end{array}\right)$ où $M$ est une $n \times n$ matrice régulière à élé. ments complexes $a_{l}^{h}$ et $M^{\prime}$ (à éléments complexes $a_{h^{\prime}}^{l^{\prime}}$ sa contragrédiente $\left(\Sigma a_{h}^{l} a_{t^{\prime}}^{l^{\prime}}=\delta_{t}^{l}\right) ; \tilde{l}_{2 n}$ est donc isomorphe a $L_{n}^{\prime}$. Le sous-groupe de $\tilde{l}_{2 n}$ engendré par les matrices invariantes par l'antiinvolution $B$ de l'espace des $n \times n$. matrices complexes correspondant à l'antiinvolution de $C^{2 n}: z^{s} \rightarrow z^{s^{\prime}}, z^{z^{\prime}} \rightarrow z^{s}$ $(s=1, \ldots, n)$ est le groupe unitaire $U_{n}$ (pour ce groupe on a: $\Sigma a_{h}^{i} a_{t}^{l}=\delta_{t}^{h}$ ). Le groupe unitaire $U_{n}{ }^{(k)}$ d'espèce $k$ est la forme réelle de $\tilde{l}_{2 n}$ correspondant à l' antiinrolution : $z^{s} \rightarrow \bar{z}^{s^{\prime}}, \quad z^{s^{\prime}} \rightarrow \bar{z}^{s}, z^{\lambda} \rightarrow-z^{\lambda^{\prime}}, z^{\lambda^{\prime}} \rightarrow-z^{\lambda} \quad(s=1, \ldots, n$; $\left.s^{\prime}=s+k ; \lambda=k+1, \ldots, n ; \lambda^{\prime}=\lambda+n\right)$. Le groupe para-nnitaire $\stackrel{\circ}{U}_{n}$ est la forme réelle de $\tilde{l}_{2,}$ correspondant à l'antiinvolution: $z^{s} \rightarrow \bar{z}^{s}, z^{s^{\prime}} \rightarrow \bar{z}^{s^{\prime}}$ $(s=1, \ldots, n)$. Donc $\stackrel{\circ}{U}_{n}$ pent être identifié au groupe des matrices $\left(\begin{array}{cc}N & 0 \\ O & N^{\prime}\end{array}\right)$ où $N$ est une $n \times n$ matrice à éléments réels et $N^{\prime}$ sa contragrédiente : le groupe

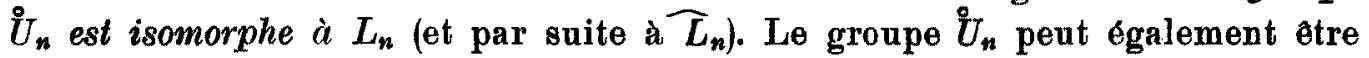
identifié an groupe des $n \times n$ matrices régulières à éléments paracomplexes $u_{h}^{l}=a_{h}^{l}+j b_{h}^{l}$ telles que: $\Sigma u_{h}^{l} \bar{u}_{t}^{l}=\delta_{t}^{h}$.

Nous arons ainsi démontré que les groupes $U_{n}, U_{n}^{\langle k\rangle}, \stackrel{O}{U}_{n}, \widehat{L}_{n}$ sont isomorphes à des formes réelles du groupe linéaire complexe $L_{n}^{\prime}$. Si $n=2 p, \widehat{L}_{z p}$ est identique au groupe linéaire quaternionien de deuxième espèce $L^{\prime \prime}{ }_{p}$; ce groupe $\stackrel{\circ}{L}_{p}$ et le groupe linéaire quaternionien $L^{\prime \prime}{ }_{p}$ sont isomorphes à des formes réelles d'un même groupe complexe.

Si l'on considère les groupes unimodulaires $S U_{n}, S U_{n}^{(k)}, S \dot{U}_{n}, S \widehat{L}_{n}$ et, si $n=2 p$, $S L^{\prime \prime}{ }_{p}, S L^{\prime \prime}{ }_{p}$, on obtient, à un isomorphisme près, toutes les formes réelles du groupe linéaire homogène complexe unimodalaire [11] (groupe simple complexe du type $A[8])$.

De mème le groupe unitaire quaternionien de deuxième espèce $\mathfrak{L}_{p}$ (isomorphe au groupe symplectique réel $\tilde{L}_{2 p}$ ) et le groupe unitaire quaternionien (isomorphe au groupe des $2 p \times 2 p$ matrices unitaires laissant invariante une forme extérieure quadratique de rang $2 p$ ) sont isomorphes a des formes rélles $\mathrm{du}$ groupe symplectique complexe à $2 p$ variables (groupe simple complexe du type $C$ ).

15. Isomorphismes associés à une forme quadratique extérieure $Q$, de rang $2 n$ [29] et [40].

Soit $\alpha$ l' isomorphisme de l'espace vectoriel $R^{2 n}$ sur son dual qui a tout vecteur $X \& R^{2 n}$ fait correspondre la forme $\alpha(X)=X . \mid \mathrm{Q}$ (où le symbole $\rfloor$ désigne le produit intérieur [6]). Le prolongement de $\alpha$ à l'ensemble des $p$-vecteurs sera encore désigné par $\alpha$ : l'isomorphisme $\alpha$ de l'espace vectoriel 
des $p$-vecteurs sur l'espace vectoriel de $p$-formes associe a tont $p$-vecteur décomposable $X_{p}=X^{1} \wedge \ldots \wedge X^{p}$, la $p$-forme décomposable $\alpha\left(X_{p}\right)=\alpha\left(X^{1}\right) \wedge$ $\wedge \ldots \wedge \alpha\left(X^{p}\right)$ (que nous désignerons aussi par forme monôme). Inversement a toute $p$-forme $\varphi_{p}$, correspond un $p$-vecteur $\alpha^{-1}\left(\varphi_{p}\right)$. A cette forme $\varphi_{p}$, on fait également correspondre une $(2 n-p)$-forme qui sera désignée par $* \varphi_{p}$ et qui sera appelée adjointe de $\varphi_{p}$ par rapport $\grave{a} \Omega$, cette forme $* \varphi_{p}$ étant définie par:

$$
\tilde{*} \varphi_{p}=\alpha^{-1}\left(\varphi_{p}\right) \perp \frac{\Omega^{n}}{n !} \text {. }
$$

L'opérateur * est un isomorphisme de l'espace vectoriel des $p$-formes sur. l' espace vectoriel des $(2 n-p)$-formes $\left({ }^{8}\right)$. On démontre la propriété suivante:

$$
* \tilde{*} \varphi_{p}=\varphi_{p} \text {. }
$$

Remarquons $q u$ 'en raison d'un théorème démontré dans [6], si $\varphi_{p}$ est décomposable il en est de même de $\tilde{*} \varphi_{p}=\alpha^{-1}\left(\varphi_{p}\right)-1 \frac{\Omega^{n}}{n !}$ puisque $\alpha^{-1}\left(\varphi_{p}\right)$ est aussi décomposable.

En raison des propriétés du produit intérieur [6], on $a, \varphi_{p}$ et $\varphi_{q}$ étant des formes de degrés $p$ et $q$ :

soit

$$
\tilde{*}\left(\varphi_{p} \wedge \varphi_{q}\right)=\left[\alpha^{-1}\left(\varphi_{p}\right) \wedge \alpha^{-1}\left(\varphi_{q}\right)\right] \perp \frac{\Omega^{n}}{n !}=\alpha^{-1}\left(\varphi_{p}\right) \perp\left[\alpha^{-1}\left(\varphi_{q}\right) \perp \frac{\Omega^{n}}{n !}\right],
$$

$$
\left.\left.\tilde{*}\left(\varphi_{p} \wedge \varphi_{q}\right)=\alpha^{-1}\left(\varphi_{p}\right)\right\rfloor\left(\tilde{*} \varphi_{q}\right)=(-1)^{p q_{\alpha}-1}\left(\varphi_{q}\right)\right\rfloor\left(\tilde{*} \varphi_{q}\right)
$$

en particulier si $\theta$ est une forme linéaire, on $a: \theta \wedge \tilde{*} \Omega=\alpha^{-1}(\theta) . I \Omega=\theta$, d'où :

$$
\tilde{*} \theta=\theta \wedge \tilde{*} \text {. }
$$

On démontre également les formules:

$$
\tilde{*} \Omega=\frac{\Omega^{n-1}}{(n-1) !}, \quad \approx \frac{\Omega^{p}}{p !}=\frac{Q^{n-p}}{(n-p) !}, \quad * \frac{Q^{n}}{n !}=1 .
$$

Soit $F$ une forme quadratique définie positive échangeable avec $\Omega$ et soient $\omega^{1}, \ldots, \omega^{2 n}$, un ensemble de $2 n$ formes lineaires linéairement indépendantes telles que :

$$
\begin{aligned}
& \Omega=\omega^{1} \wedge \omega^{n+1}+\ldots+\omega^{n} \wedge \omega^{2 n}, \\
& F=\left(\omega^{1}\right)^{2}+\left(\omega^{n+1}\right)^{2}+\ldots+\left(\omega^{n}\right)^{2}+\left(\omega^{2 n}\right)^{2} .
\end{aligned}
$$

Soit $\mathfrak{J}$ l'automorphisme de $R^{2 n}$, produit des dualités par rapport à $\Omega$ et $F$. L'antomorphisme $C$, contragrédient de $\mathfrak{J}$ dans le dual de $R^{2 n}$, se prolonge aux $p$-formes. On a, en particulier: $C \omega^{j}=\omega^{j+n}, C \omega^{j+n}=-\omega^{j}$ et, en posant $\theta^{j}=\omega^{j}+i \omega^{j+n}$, on obtient : $C \theta^{j}=-i \theta^{j}, C \bar{\theta}^{j}=i \bar{\theta}^{j}$.

(8) L'opérateurs * est l'opérateur désigné par * dans [40]. 
Cet opérateur $C$, utilisé par GJGGENHErmen [31], est au signe près, l'opérateur $C$ introduit par A. WEIL [46].

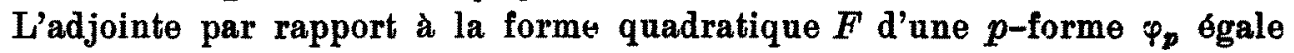
aे $\Sigma A_{\beta_{1} \ldots \beta_{p}} \omega^{\beta_{1}} \wedge \ldots \wedge \omega^{\beta p}$, est par définition [42]:

$$
* \varphi_{p}=\Sigma \delta_{\beta_{1} \ldots \beta_{2 n}}^{1 \ldots .2 n} A_{\beta_{1} \ldots \beta_{p}} \omega^{\beta_{1}+1} \wedge \ldots \wedge \omega^{\beta_{2 n}} .
$$

On a, entre $* \varphi_{p}$ et $* \varphi_{p}$, la relation :

$$
\tilde{*} \varphi_{p}=(-1)^{\frac{n(n-1)}{2}+p} * C \varphi_{p} .
$$

Introduisons l'opérateur $L$ et ses adjoints $\tilde{\Lambda}$ et $\Lambda$ par rapport à $\Omega$ et $F$ :

$$
L \varphi_{p}=\varphi_{p} \wedge \Omega, \quad \tilde{\Lambda} \varphi_{p}=\tilde{*} L \tilde{*} \varphi_{p}, \quad \Lambda \varphi_{p}=(-1)^{p} * L * \varphi_{p} .
$$

En raison de (15.7): $\tilde{\Lambda} \varphi_{p}=* C\left(* C \varphi_{p} \wedge \Omega\right)=*\left(C^{\varepsilon} * \varphi_{p} \wedge \Omega\right)=(-1)^{p_{*}} L *^{*} \varphi_{p}$ car $* C=C *$. Done :

$$
\tilde{\Lambda} \varphi_{p}=\Lambda \varphi_{p}
$$

L'opérateur $\Lambda$, identique $\dot{a} \tilde{\Lambda}$, ne dépend que de $\mathbf{Q}$ et non de la forme quadratique échangeable avec $\mathbf{\Omega}$. On utilisera désormais uniquement le symbole $\Lambda$. Remarquons qu' en raison de (15.3):

$$
\Lambda \varphi_{p}=\alpha^{-1}(\Omega) \perp \varphi_{p} .
$$

Nous supposerons désormais $p \leq n$. Une $p$-forme $\varphi_{p}$ sera dite effective ou de classe 0 si $\Lambda \varphi_{p}=0$, c'est-à-dire si $\left.\alpha^{-1}(\Omega)\right\rfloor \varphi_{p}=0$. Une $p$-forme sera dite de classe $k$ si $\varphi_{p}=\psi_{p-2 k} \wedge \frac{Q^{k}}{k !}$ où $\psi_{p-2 k}$ est effective. Une forme de classe déterminê sera dite simple.

Par répétition de l'opérateur $\Lambda$ on obtient les opérateurs: $\Lambda^{2}, \ldots, \Lambda^{\left[\frac{n}{2}\right]}$. Pour une $p$-forme $\varphi_{p}$, on $a: \Lambda^{\left[\frac{p}{q}\right]+1} \varphi_{p}=0$. Par conséquent à toute $p$-forme on peut associer un nombre $l$ bien déterminé compris entre $O$ et $\left[\frac{p}{2}\right]$ que nous appellerons son genre tel que: $\Lambda^{l+1} \varphi_{p}=0, \Lambda^{l} \varphi_{p} \neq 0$. La forme $\Lambda^{l} \varphi_{p}$ est donc effective; (si $\varphi_{p}$ est effective, $l=0$ ). On peut de même définir les opérateurs $\Lambda_{2}, \ldots, \Lambda_{s} \ldots, \Lambda_{\left[\frac{n}{2}\right]}$ tels que :

$$
\left.\Lambda_{s} \varphi_{p}=\tilde{*} L^{*} * \varphi_{p}=\alpha^{-1}\left(Q^{z}\right)\right\rfloor \varphi_{p} \quad\left(s=1, \ldots,\left[\frac{n}{2} \mid\right) .\right.
$$

ThÉonìme 15.1. - L'adjointe par rapport à $\Omega$ d'une forme effective $\psi_{p}$ est:

$$
\tilde{*} \psi_{p}=\varepsilon_{p} \psi_{p} \wedge \frac{\mathrm{Q}^{n-p}}{(n-p) !}, \text { où } \quad \varepsilon_{p}=(-1)^{\frac{p(p-1)}{\varepsilon}} \text {. }
$$


On démontre d'abord le théorème pour les formes monômes. La forme $\mathbf{\Omega}$ étant réduite à la forme canonique $\Sigma \omega^{j} \wedge \omega^{j+n}$, pour qu' une forme $\Pi_{p}$ monòme par rapport aux $\omega^{8}$ soit effective, il faut et il suffit qu'elle ne renferme aucun couple $\mathrm{d}$ 'indices differant de $n$. Pour une telle forme $\Pi_{p}=A_{\beta_{1} \ldots \xi_{p}} \omega^{\beta_{1}} \wedge \ldots \wedge \omega^{z_{p}}$ on a $: \alpha^{-1}\left(\Pi_{p}\right)=A_{\beta_{1} \ldots \beta_{p}} e_{\beta_{1} \div n} \wedge \ldots \wedge e_{\beta_{p} \div n}$, où les $e^{\beta}$ forment la base duale des $\omega^{\tilde{F}}$, avec $\beta+2 n=-\beta$, d' où :

$$
\begin{aligned}
& \tilde{*} \Pi_{p}=(-1)^{\frac{\left.p^{\prime} p-1\right\rangle}{2} \omega^{\beta_{1}}} \wedge \ldots \wedge \omega^{3 p} \wedge \omega_{1 / 1}^{r_{1}} \wedge \omega^{\gamma_{1}+n} \wedge \ldots \wedge \omega^{\gamma_{n-p}} \wedge \omega^{\gamma_{n-p}+n} \\
& =(-1)^{\frac{\left.p^{\prime} p-1\right\rangle}{2} \omega^{\hat{k}}} \wedge \cdots \wedge \omega^{\hat{R}_{p}} \wedge \frac{\mathbf{Q}^{n-p}}{(n-p) !} .
\end{aligned}
$$

Si la forme effective $\}_{p}$ n'est pas monôme, on démontre le théorème en décomposant $\psi_{p}$ en la somme:

$$
\begin{gathered}
\Pi_{p}+\Sigma\left(\omega^{j} \wedge \omega^{j+n}-\omega^{l} \wedge \omega^{l+n}\right) \wedge \Pi_{p-2}^{j l}+ \\
+\Sigma\left(\omega^{j} \wedge \omega^{j+n}-\omega^{l} \wedge \omega^{l+n}\right) \wedge\left(\omega^{h} \wedge \omega^{n+n}-\omega^{s} \wedge \omega^{s+n}\right) \wedge \Pi_{p-4}^{i l h s}+\ldots,
\end{gathered}
$$

oü les formes II sont des sommes de monômes effectifs.

Corollatre. - Soit $\varphi_{p}=\psi_{p-2 k} \wedge \frac{\mathbf{Q}^{k}}{k !}$ une p-forme de classe $k ;$ son adjointe est égale à :

$$
\begin{aligned}
\tilde{*}^{*} \varphi_{p} & =\varepsilon_{p-2 k} \psi_{p-2 k} \wedge \frac{Q^{n-p+k}}{(n-p+k) !} \\
& =(-1)^{k} \varepsilon_{p} \varphi_{p} \wedge \frac{Q^{n-p}}{(n-p) !} \frac{k !}{(n-p+k) !} .
\end{aligned}
$$

On en déduit les formules suivantes, relatives à la forme simple ${ }_{p}$ :

$$
\begin{aligned}
& \Lambda_{F_{p}}=\frac{(n-p+k+1) !}{(n-p+k) !} \Downarrow_{p-2 k} \wedge \frac{\Omega^{k-1}}{(k-1) !}, \\
& \Lambda^{2} \psi_{p}=\Lambda_{2} \psi_{p}=\frac{(n-p+k+2) !}{(n-p+k) !} \psi_{p-2 k} \wedge \frac{Q^{k-2}}{(k-2) !}, \\
& \Lambda^{k_{p_{p}}}=\Lambda_{k} \hat{\psi}_{p}=\frac{(n-p+2 k) !}{(n-p+k) !} \psi_{p-2 k}, \\
& \Lambda^{k+1} \varphi_{p}=\Lambda_{k+1} \varphi_{p}=0 \text {. }
\end{aligned}
$$

Pour une forme simple le genre est donc égal à la classe.

THÉnĖye 15.2. - Toute forme $\varphi_{p}$ de degré $p$ se décompose de manière unique en la somme :

$$
\psi_{p}=\psi_{p}+\psi_{p-2} \wedge \mathrm{Q}+\ldots+\psi_{p-2 q} \wedge \frac{\mathrm{Q}^{q}}{q !} \text { avec } q \leq\left[\frac{p}{2}\right],
$$

les formes $\psi_{p-z h}$ étant effectives (ou nulles). 
Ce theorème de décomposition est da a Eckmann-Guggenhermer [22] mais nous en donnons une démonstration différente: soit $q$ le genre de $\varphi_{p}$; on peut poser $\Lambda^{q} \varphi_{p}=\frac{(n-p+2 q) !}{(n-p+q) !} \psi_{p-2 q}$ où $\psi_{p-2 q}$ est effective; en raison des formules (15.14), la forme $\varphi_{p}-\psi_{p-2 q} \wedge \frac{\mathbf{Q}^{q}}{q !}$ est de genre inferieur ou égal à $q-1$ et l'on peut poser: $\Lambda^{q-1}\left(\varphi_{p}-\psi_{p-2 q} \wedge \frac{\Omega^{q}}{q !}\right)=\frac{(n-p+2 q-2) !}{(n-p+q-1) !} \psi_{p-2 q+2}$ où $\psi_{p-2 q+2}$ est effective ou nulle; on définit ainsi de proche en proche des formes effectives $\psi_{p-2 q}, \ldots, \psi_{p-2}, \psi_{p}$ et l'on obtient la décomposition de $\varphi_{p}$ en formes simples; en raison de la signification des formes $\psi_{p-2 h}$, cette decomposition est unique.

On déduit du théorème 15.2 la décomposition de $\tilde{*} \varphi_{p}$; la forme $* \varphi_{p}$ se décompose de manière unique en la somme:

$$
\begin{gathered}
\tilde{*} \varphi_{p}=\varepsilon_{p}\left[\psi_{p}-\psi_{p-2} \wedge \frac{\Omega}{n-p+1}+\ldots\right. \\
\left.+(-1)^{q} \psi_{p-q} \wedge \frac{\Omega^{q}}{(n-p+1) \ldots(n-p+q)}\right] \wedge \frac{Q^{n-p}}{(n-p) !}
\end{gathered}
$$

ce qui permet de démontrer le théorème suivant:

ThÉonìme 15.3. - L'opérateur $f$ tel que $f\left(\varphi_{p}\right)=\frac{1}{(n-p) !} \approx L^{n-p_{\varphi}} \varphi_{p}$ est un automorphisme de l'espace vectoriel des $p$-formes aux valeurs propres duquel correspondent les formes simples.

La formule (15.16) peut s'ecrire: $\tilde{*} \varphi_{p}=f^{-1}\left(\varphi_{p}\right) \wedge \frac{\Omega^{n-p}}{(n-p) !}$. Pour toute forme $\varphi_{n+p}$, on $a: \varphi_{n+p}=f^{-1}\left(\tilde{*} \varphi_{n+p}\right) \wedge \frac{\Omega^{p}}{p !}$ ce qui exprime le théorème de LEPAGE [35].

Autre énoncé du théorème 15.3: l'application qui à tout p-vecteur $X_{p}$ fait correspondre la forme $X_{p} \frac{\Omega^{p}}{p}$ ! est un isomorphisme de l'espace vectoriel des $p$-formes sur son dual. On a en effet: $\tilde{*}\left(\varphi_{p} \wedge \frac{Q^{n-p}}{(n-p) !}\right)=\alpha^{-1}\left(\varphi_{p}\right) \perp \frac{Q^{p}}{p !}$.

Conséquences du théorème 15.3.

$1^{\circ}$ Pour qu'une forme $\psi_{p}$ soit effective, il faut et il suffit que:

$$
\psi_{p} \wedge \mathbf{Q}^{n-p+1}=0 \text {. }
$$

La décomposition en formes simples est donc identique à la décomposition de Lepage.

$2^{\circ}$ Pour $q u^{\prime} u n e$ forme $\varphi_{p}$ soit de genre $q$, il faut et il suffit que :

$$
\begin{aligned}
& \varphi_{p} \wedge \Omega^{n-p+q+1}=0 \\
& \varphi_{p} \wedge \Omega^{n-p+q} \neq 0 .
\end{aligned}
$$


$3^{\circ}$ Si deux formes $\varphi_{p}$ et $\varphi_{p}^{\prime}$ sont de même degré $p$, on a:

$$
\varphi_{p} \wedge \tilde{*} \varphi_{p}^{\prime}=(-1)^{p} \varphi_{p}^{\prime} \wedge \cdot \tilde{*} \varphi_{p}
$$

En particulier si $p$ est impair,

$$
\varphi_{p} \wedge \tilde{*} \varphi_{p}=0
$$

Ces trois propriétés se démontrent en utilisant la décomposition en formes simples et le théorème de Lepage. Remarquons $q u$ 'en raison du théorème de décomposition et des formules (15.14) les opérateurs $\Lambda_{\text {, et }} \Lambda^{\text {s }}$ sont identiques quelle que soit la forme $\varphi_{p}$.

Ce théorème et ces formules permettent également de démontrer le théorème suivant:

Théonǵme 15.4. - L'opérateur $\Lambda^{k} L^{k}(1 \leq k \leq n-1)$ est un automorphisme de l'espace vectoriel des $p$-formes, aux valeurs propres duquel correspondent les formes simples $(p=1, \ldots, n-k)$.

Eokmann et Guggenhermer ont d'ailleurs démontré que $\Lambda L$ est un automorphisme de l'espace vectoriel des formes de degré $p \leq n-2$.

Chapitre IV.

\section{Structures presque symplectiques.}

La plupart des résultats de ce chapitre ont été exposés dans [29], [40]. Les notations sont différentes: les opérateurs désignés par $*$ et $\dot{\delta}$ dans [40] seront désignés respectivement par $\tilde{*}$ et $\tilde{\delta}$ (cf. $\S 15$ ).

16. Définitions,

La donnée sur une variété $V_{2 n}$, de dimension $2 n$ (qui sera supposée deux fois différentiable) d'une forme différentielle extérieure quadratique $\Omega$, différentiable, de rang $2 n$ en tout point $x \& V_{2 n}$, définit sur $V_{2 n}$ une structure que nous appellerons presque symplectique (c'est-à-dire une structure infinitésimale régulière dont le groupe structural est le groupe symplectique $\tilde{L}_{n}$ ). Si la forme $\Omega$ est fermée, e' est-à-dire si la différentielle $d \Omega$ est nulle, la structure est dite symplectique, suivant la définition donnée par C. EHRESMANN [24].

En chaque point $x$, la restriction $\Omega_{x}$ de $\Omega$ à l'espace tangent $T_{x}$ à $V_{2 n}$ détermine un isomorphisme $\alpha_{x}$ (défini dans $\S 15$ ) de $T_{x}$ sur son dual, isomorphisme que l'on peut prolonger à l'ensemble des $p$-vecteurs. Le champ de ces isomorphismes fait correspondre à tout champ $X$ de $p$-vecteurs sur $V_{2 n}$ une forme différentielle extérieure qui sera désignée par $\alpha(X)$. Inversement à toute forme differentielle extérieure $\varphi_{p}$, de degré $p$, correspond un champ de vecteurs $\alpha^{-1}\left(\varphi_{p}\right)$. On peut ainsi définir l'adjointe d'une forme différentielle extérieure par rapport à $\Omega$, ainsi que l'opérateur $\Lambda$, d' où la notion de classe d' une forme differentielle extérieure. 
Si l'on se donne sur $V_{2 n}$, outre la forme $\mathbf{Q}$, une métrique riemannienne $F$ définie positive, échangeable avec $Q$, on détermine sur $V_{2 n}$ une structure presque hermitienne, structure qui sera étudiée ultérieurement (\$22). Soit dans l'espace tangent $T_{x}$ à $V_{2 n}$, l'automorphisme $\mathfrak{J}_{x}$, produit des dualités par rapport à $\boldsymbol{Q}_{x}$ et $F_{x}$ et soit $C_{x}$ le contragrédient de cet automorphisme dans le dual de $T_{x}$. Nous désignerons par $C \varphi_{p}$ la forme différentielle extérieure dont la restriction à $T_{x}$ est la transformée par $C_{x}$ de la restriction de $\varphi_{p}$.

Par définition, la codifférentielle d'une forme $\varphi_{p}$ relativement à $\mathbf{Q}$ est la forme $\tilde{\delta} \varphi_{p}$, de degré $p-1$, définie par :

$$
\tilde{\delta} \varphi_{p}=\tilde{*} d \tilde{*} \varphi_{p} \text {. }
$$

On a: $\tilde{\delta} \tilde{\delta} \varphi_{p}=0$.

La codifférentielle d'une forme $\varphi_{p}$ par rapport à la métrique rieman. nienne $F$ est par definition [31]:

$$
\delta \varphi_{p}=-* d * \varphi_{p} .
$$

De la relatịon (15.7), on déduit:

$$
\tilde{\delta} \varphi_{p}=\tilde{*} d \tilde{*} \varphi_{p}=-C * d * \varphi_{p}=C \delta C \varphi_{p},
$$

soit :

$$
\tilde{\delta} \varphi_{p}=(-1)^{p} C^{-1} \delta C \varphi_{p} .
$$

Une forme $\varphi_{p}$ telle que $d \varphi_{p}=0, \tilde{\delta} \varphi_{p}=0$, sera dite harmonique par rapport $\dot{a} \Omega$.

Supposons la variété $V_{2 n}$ compacte. On peut alors définir le produit scalaire de deux formes $\varphi_{p}$ et $\theta_{p}$ de même degré par:

$$
\left(\varphi_{p}, \theta_{p}\right)=\int_{V_{2 n}} \varphi_{p} \wedge * \theta_{p} .
$$

Le carré scalaire $\left(\varphi_{p}, \varphi_{p}\right)$ peut être nul sans que $\varphi_{p}=0$, en particulier si $p$ est impair, il est nul pour toute forme $\varphi_{p}$.

Soient $\varphi_{p}$ et $\theta_{p+1}$ deux formes de degrés $p$ et $p+1$. On a: $\left(d \varphi_{p}, \theta_{p+1}\right)=$ $=(-1)^{p+1}\left(\varphi_{p}, \tilde{\delta}_{p+1}\right) \operatorname{car} \int_{V_{2 n}} d\left(\varphi_{p} \wedge \tilde{*}_{p+1}\right)=0$. En particulier:

$$
\left(\varphi_{p}, \tilde{\delta} d \varphi_{p}\right)=(-1)^{p+1}\left(d \varphi_{p}, d \varphi_{p}\right) \text { et }\left(\varphi_{p}, d \tilde{\delta} \varphi_{p}\right)=(-1)^{p}\left(\tilde{\delta} \varphi_{p}, \tilde{\delta} \varphi_{p}\right)
$$

Donc si $\tilde{\delta} d \varphi_{p}=0$, on a: $\left(d \varphi_{p}, d \varphi_{p}\right)=0$, ce qui n'entraîne pas nécessairement $d \varphi_{p}=0$.

Contrairement aux formes harmoniques par rapport à une métrique riemannienne, une forme harmonique relativement à $\Omega$ pent être homologue ou cohomologue à 0 sans être nulle.

Dans la suite la variété $V_{2 n}$ n' est pas nécessairement compacte. 
17. Covariants associés à une strueture presque symplectique.

Soit sur une variété $V_{2 n}$ une structure presque symplectique définie par la donnée d'une forme différentielle exterieure quadratique $\mathbf{Q}$. On obtient un covariant [26] en considérant la codifférentielle $\tilde{\delta} \Omega$ de la forme par rapport à elle-même :

$$
\tilde{*} \tilde{\Omega}=d \tilde{*} \Omega=d \Omega \wedge \frac{\Omega^{n-2}}{(n-2) !}
$$

or $\tilde{\delta} \Omega$ étant de degré $1, \tilde{*} \tilde{\delta} \Omega=\tilde{\delta} \Omega \wedge \tilde{*} \Omega=\tilde{\delta} \Omega \wedge \frac{\Omega^{n-1}}{(n-1) !}$, en raison de (15.4). D' où :

$$
\left(d \boldsymbol{Q}-\frac{\tilde{\delta} \boldsymbol{\Omega} \wedge \mathrm{Q}}{n-1}\right) \wedge \mathbf{\Omega}^{n-2}=0
$$

Nous supposerons d'abord $n>2$. En raison du premier corollaire du théorème (15.3), cette dernière relation est équivalente à :

$$
d \mathbf{Q}=\psi+\frac{\tilde{\delta} \mathbf{Q} \wedge \mathbf{\Omega}}{n-1}
$$

où $\psi$ est effective.

On obtient ainsi la décomposition de dQ en formes simples, d'où, en nti. lisant les formules $(15.14)$ et $(15.10)$ :

$$
\left.\tilde{\delta} \Omega=\Lambda d Q=\alpha^{-1}(\Omega)\right\rfloor d \Omega .
$$

Cette formule identifie $\tilde{\delta} \Omega$ au vecteur covariant de courbure de $\Omega$, introduit par LEE [33], et la formule (17.3) identifie la forme $\psi$ au «deuxième tenseur conforme de courbure». Nous désignerons respectivement les formes $\tilde{\delta} \Omega$ et $\psi$ par forme de torsion et forme de torsion conforme, définitions qui seront justifiées nltérieurement (cf. § 18).

En utilisant la décomposition de $d \Omega$, on peut démontrer les formules suivantes :

$$
\begin{aligned}
& \frac{\tilde{\delta} \Omega^{p}}{p !}=-\frac{d \Omega^{p-1}}{(p-1) !}+\frac{\tilde{\delta} \Omega \wedge \Omega^{p-1}}{(p-1) !} \\
& \alpha^{-1}\left(\frac{\Omega^{p}}{p !}\right)-1 d \frac{\Omega^{p}}{p !}=\Lambda^{p} d \frac{\Omega^{p}}{p !}=\frac{(n-2) !}{(p-1) !(n-p+1) !} \tilde{\delta} \Omega, \\
& \tilde{*} d \Omega=-\frac{d \Omega^{n-2}}{(n-2) !}+\tilde{\delta} \Omega \wedge \frac{\Omega^{n-2}}{(n-2) !} \\
& \tilde{*} d \Omega=\frac{d \tilde{\delta} \Omega \wedge \Omega^{n-2}}{(n-2) !}-\tilde{\delta} \Omega \wedge d \Omega \wedge \frac{\Omega^{n-3}}{(n-3) !} .
\end{aligned}
$$

Par différentiation de (17.3), on montre que $\tilde{d \delta} Q$ est effective, d'où : $\tilde{*} \tilde{\delta} d \Omega \wedge \Omega=0$ et $\tilde{\delta} d \Omega$ est effective également. La formule (17.8) peut alors 
s'écrire : $\tilde{*} \tilde{\delta} d \Omega=-\tilde{*} d \tilde{\delta} \Omega-\tilde{\delta} \Omega \wedge d \Omega \wedge \frac{\Omega^{n-3}}{(n-3) !}$, ou en introduisant l' ope. rateur $\tilde{\Delta}=d \tilde{\delta}+\tilde{\delta} d:$

$$
\tilde{*} \tilde{\Delta}=\frac{\tilde{\Delta} \Omega^{n-1}}{(n-1) !}=d \Omega \wedge \tilde{\delta} \Omega \wedge \frac{Q^{n-3}}{(n-3) !} .
$$

On a de mème (si $p>1)$ :

$$
\approx \tilde{\Delta} \frac{\Omega^{p}}{p !}=\tilde{\Delta} \frac{\Omega^{n-p}}{(n-p) !}=d \Omega \wedge \tilde{\delta} \Omega \wedge \frac{\Omega^{n-p-2}}{(n-p-2) !}+\tilde{*}\left(d \Omega \wedge \tilde{\delta} \Omega \wedge \frac{Q^{p-2}}{(p-2) !}\right)
$$

$\tilde{\Delta} \Omega$ étant effective, $\tilde{*} \tilde{\Delta} \Omega=-\tilde{\Delta} \Omega \wedge \frac{Q^{n-z}}{(n-2) !}$; on en déduit, on raison de $(179)$ : $d \Omega \wedge \tilde{\delta} \Omega+\frac{\tilde{\Delta} \Omega \wedge \Omega}{n-2}=\psi_{4}$ où $\psi_{4}$ est effective, d'où la décomposition de $d \Omega \wedge \tilde{\varepsilon} \Omega$ en formes simples:

$$
d \Omega \wedge \tilde{\delta} \Omega=\psi_{4}-\frac{\tilde{\Delta} \mathbf{\Omega} \Lambda \mathbf{Q}}{n-2}
$$

la formule (17.10) peut s'écrire:

$$
\tilde{*} \tilde{\Delta} \frac{Q^{p}}{p !}=\tilde{\Delta} \frac{Q^{n-p}}{(n-p) !}=\frac{2 d \Omega \wedge \tilde{\delta} \Omega \wedge \Omega^{n-p-z}}{(n-p-2) !}+\frac{\tilde{\Delta} Q \wedge \Omega^{n-p+1}}{(n-p+1) !}
$$

ou encore :

$$
\tilde{\Delta} \frac{\Omega^{p}}{p !}=\frac{2 d \Omega \wedge \tilde{\delta} \Omega \wedge \Omega^{p-2}}{(p-2) !}+\frac{\tilde{\Delta} \Omega \wedge \Omega^{p-1}}{(p-1) !}
$$

On en déduit : $\Lambda^{p} \tilde{\Delta} \Omega^{p}=0$ et $\tilde{\Delta} \Omega^{p}$ est de genre $p-1$.

Si $\tilde{\delta} \Omega=0$, on a : $d \tilde{\delta} \Omega^{p}=\tilde{\delta} d \Omega^{p}=\tilde{\Delta} \Omega^{p}=0$, quel que soit $p$. Si $\tilde{\delta} \Omega \neq 0$, comme $\tilde{\delta} \Omega \wedge \tilde{*} \tilde{\Delta} \Omega=0$, soit en raison de $(15.3): \alpha^{-1}(\tilde{\delta} \Omega) . \mathbf{I} \tilde{\Delta} \Omega=0$, il en résulte que $\tilde{\Delta} \Omega$ est de rang inférieur à $2 n$.

En appliquant plusieurs fois les opérateurs $d, \tilde{\delta}, \tilde{\Delta}, \Lambda$, on obtient un ensemble de covariants en général non nuls; si parmi eux il y a $2 n$ formes linéaires indépendantes on peut mettre $\Omega$ sous une forme canonique et le problème d'équivalence se ramène au problème d'équivalence restreint. Si $\tilde{\delta} \Omega=0$, tous les invariants obtenus précédemment sont nuls et le problème d'équivalence ne peut être résolu par les mêmes méthodes.

Supposons maintenant $n=2$. On a alors: $\approx \Omega=\Omega$, d'où $\approx d \Omega=d \Omega$ et $d \Omega=\tilde{\delta} \Omega \wedge \Omega$, relation qui résulte également de (17.2). Par différentiation, on obtient: $\tilde{d} \delta \Omega \wedge \mathbf{Q}$, done $\tilde{d} \delta \Omega$ est effective et $\tilde{*}(\tilde{d} \tilde{\delta} \Omega)=-d \tilde{\delta} \Omega$. D'autre part $\tilde{*}(\tilde{\delta} d \Omega)=d \tilde{*} d \Omega=\tilde{d} \tilde{\delta} \Omega$. Done $\tilde{\Delta} \Omega=0$.

Si la forme $\Omega$ est cofermée $(\tilde{\delta} \Omega=0)$, elle est également fermée $(d \Omega=0)$, contrairement à ce qui a lieu en général pour $n$ quelconque. 
Le problème d'équivalence des formes différentielles extérieures quadratiques à 4 variables a été traité par YEN-CHIH-TA [47]. Dans le cas le plus général (celui où $d \tilde{\delta} \Omega$ est de rang 4), $\Omega$ et dõ $\Omega$ peuvent se mettre sous la forme canonique:

$$
\begin{gathered}
\Omega=\omega^{1} \wedge \omega^{2}+\omega^{3} \wedge \omega^{4} \\
d \tilde{\delta} \Omega=\lambda\left(\omega^{1} \wedge \omega^{2}-\omega^{3} \wedge \omega^{4}\right),
\end{gathered}
$$

les formes $\omega^{1}, \omega^{2}, \omega^{3}, \omega^{4}$ étaut entièrement déterminées par les conditions:

$$
\tilde{\delta} \mathbf{Q}=\omega^{1}+\omega^{3}, \quad d \lambda=\lambda_{2} \omega^{2}+\lambda_{4} \omega^{4} \quad\left(\lambda_{2} \lambda_{4} \neq 0\right) .
$$

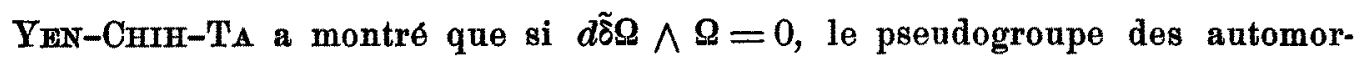
phismes de la structure est un pseudogroupe infini de LIE; en dehors de ce cas, il n'a traité que ceux pour lesquels on peut déterminer canoniquement 4 formes de Pfafr.

18. Formes $\Omega$ admettant un facteur intégrant.

Si l'on multiplie la forme $\Omega$ par une fonction différentiable $\lambda$, on peut définir la codifférentielle $\tilde{\delta}^{\prime} \Omega^{\prime}$ de la forme $\Omega^{\prime}=\lambda \Omega$ par rapport à elle-même. On a : $d \Omega^{\prime}=\lambda d \Omega+\frac{d \lambda}{\lambda} \wedge \Omega^{\prime} ; d^{\prime}$ où : $d \Omega^{\prime}=\psi^{\prime}+\frac{\tilde{\delta^{\prime}} \Omega^{\prime} \wedge \Omega^{\prime}}{(n-1)}$, avec :

$$
\begin{aligned}
& \psi^{\prime}=\lambda \psi \\
& \tilde{\delta}^{\prime} \Omega=\tilde{\delta} \Omega+(n-1) \frac{d \lambda}{\lambda} .
\end{aligned}
$$

Par suite $: \tilde{d \tilde{\delta} \Omega}=\tilde{d \tilde{\delta}} \mathbf{\Omega}^{\prime}(\tilde{d} \tilde{\delta} \Omega$ est le "premier tenseur covariant de courbure» de LEE).

Dono si l'on multiplie la forme $\Omega$ par une fonction différentiable $\lambda$, le "premier tenseur covariant de courbure est invariant et la forme de torsion conforme est multipliée par $\lambda$, ce qui justifie le terme "conforme».

Pour que la forme $\Omega$ admette un facteur intégrant, c'est-à-dire pour $q u$ 'il existe une fonction $\lambda$ telle que $d(\lambda \Omega)=0$, il fant que $\psi=0$ et $\bar{d} \bar{\delta} \Omega=0$. Réciproquement si $\psi=0$, on $a: d \Omega=\frac{\tilde{\delta} \Omega \wedge \Omega}{n-1}$ et $d \tilde{\delta} \Omega \wedge \Omega=0$, ce qui entraine $d \tilde{\delta} \Omega=0$, si $n>2$; il existe alors une fonction $\lambda$ telle que localement: $\tilde{\delta} \Omega+(n-1) \frac{d \lambda}{\lambda}=0$, d'où $d(\lambda \Omega)=0$ en raison des formules $(18.1) ;$ si $n=2$, $\psi=0$ identiquement et la condition cherchée est : $\tilde{d \tilde{\delta}} \Omega=0$. On obtient ainsi le théorème suivant [29] démontré également par LEe d'une manière différente:

Thrómine 18.1. - Pour que la forme $\mathbf{Q}$ admette un facteur intégrant local, il faut et pour $n>2$, il suffit que la forme de torsion conforme soit nulle (ou encore que la forme $d \Omega$ soit une forme de classe 1): ce qui entraine $d \tilde{\delta} \Omega=0$. Si $n=2$, il faut et il suffit que la codifférentielle $\tilde{\delta} \Omega$ soit une forme fermée. 
Remarquons que le facteur intégrant n'est défini qu'à un facteur constant près. Si la forme $\tilde{\delta} \Omega$ est homologue à 0 , la forme $\Omega$ admet un facteur inté. grant global.

Si la forme $\boldsymbol{\Omega}$ est fermée, il existe dans le voisinage $U$ de tout point de $V_{i n}$ des systèmes de coordonnées locales $x^{1}, \ldots, x^{n}, y^{1}, \ldots, y^{n}$ tels que cette forme puisse s' ecrire :

$$
\Omega=d x^{1} \wedge d y^{1}+d x^{2} \wedge d y^{2}+\ldots+d x^{n} \wedge d y^{n} .
$$

En effet soit $\omega$ une forme de PFAFF telle que dans le voisinage $U$, on ait $d \omega=\Omega$. On démontre que $\omega$ peut s'écrire: $x^{1} d y^{1}+\ldots+x^{n} d y^{n}$ et par suite $\Omega$ peut s'écrire sous la forme (18.2). Voir par exemple Thomas [44].

Si la forme $\Omega$ admet un facteur intégrant $\lambda$, on peut choisir le système de coordonnées locales de façon que $y^{1}=\frac{1}{\lambda}$ et que $\Omega$ puisse s'écrire au voisinage d'un point où $d \Omega \neq 0$ :

$$
\Omega=y^{1}\left(d x^{1} \wedge d y^{1}+d x^{2} \wedge d y^{2}+\ldots+d x^{n} \wedge d y^{n}\right)
$$

Dans les deux cas, le psendogroupe des automorphismes locaux de la structure est un pseadogroupe de LIE de type infini.

Lorsque les conditions du théorème 18.1 sont réalisées, $d \Omega$ étant divisible par $\Omega$, tout élément intégral de $\Omega$ est également élément intégral de $d \Omega$; il existe alors une variété intégrale de dimension $n$ tangente à un élément intégral arbitraire (de dimension $n$ ) de $\mathbf{\Omega}$. La forme $\Omega$ est dite complètement intégrable. Réciproquement ponr que $\Omega$ soit complètement intégrable il est nécessaire que tont élément intégral de $\mathbf{\Omega}$ soit aussi élément intégral de $d \mathbf{Q}$, et d'après un théorème de LEPage et PaPY [34] il est nécessaire que $d \Omega$ soit divisible par $\Omega$, c' est-à-dire si $n>2$, d'après le théorème 18.1 que $\Omega$ admette un facteur intégrant (ou soit fermée) puisque la forme $\psi$ est alors nulle.

Donc pour $n>2$, si une forme différentiable $Q$ (de rang $2 n$ ) est complète. ment intégrable, elle est localement équivalente à l'une des deux formes (18.2) ou (18.3). Pour $n=2$ toute forme $\Omega$ de rang 4 est complètement intégrable.

19. Torsion associée à une structure presque symplectique.

Dans ce paragraphe (dont les résultats n' ont pas été publiés), nous sup: poserons que les indices grecs varient de 1 à $2 n$, les indices latins de 1 à $n$, et que $j^{\prime}=j+n$.

Dans le voisinage $U$ d'un point de $V_{2 n}$, une structure presque symplectique peut être déterminée par la donnée de $2 n$ formes de Pfafr linéaire. ment indépendantes $\omega^{1}, \ldots, \omega^{2 n}$ telles que la forme différentielle extérieure définissant la structure puisse s'écrire :

$$
\Omega=\Sigma \omega^{j} \wedge \omega^{\prime \prime}
$$


La même structure peut être déterminée par les formes $\tilde{\omega} r=\Sigma u_{\beta}^{\gamma} \omega^{\ell}$ où les $u_{\beta}^{\gamma}$ sont des fonctions locales différentiables définissant on chaque point une transformation du groupe symplectique $\tilde{L}_{2 n}$.

$$
\begin{aligned}
& \Sigma\left(u_{j}^{k} u_{l}^{k \prime}-u_{l}^{k} u_{j}^{k^{\prime}}\right)=0, \\
& \Sigma\left(u_{j^{\prime}}^{k} u_{l^{\prime}}^{k \prime}-u_{l^{\prime}}^{k} u_{j^{\prime}}^{k^{\prime}}\right)=0, \\
& \Sigma\left(u_{j}^{k} u_{l^{\prime}}^{k^{\prime}}-u_{l}^{k} u_{j}^{k}\right)=\delta l .
\end{aligned} \quad(j, k, l=1, \ldots, n) .
$$

La restriction à tout point $x$ de $V_{2 n}$ des formes $\tilde{\omega}^{*}$ sera appelée «corepère symplectique».

Une connexion affine associée à cette structure (cf. § 6) est déterminée par l'ensemble des formes $\omega^{\beta}$ et de $4 n^{2}$ formes $\omega_{\gamma}^{\beta}$ vérifiant le relations (cf. $\S(6.4))$ :

$$
\omega_{j}^{l}+\omega_{l^{\prime}}^{j^{\prime}}=0, \quad \omega_{l}^{j^{\prime}}=\omega_{j}^{l^{\prime}}, \quad \omega_{l^{\prime}}^{j}=\omega_{j^{\prime}}^{l}, \quad(l, j=1, \ldots, n) .
$$

Les formules $(6.11)^{\prime}$ peuvent alors $\mathrm{s}^{\prime}$ écrire :

$$
\begin{aligned}
& d \omega^{j}=\Sigma \omega^{l} \wedge \omega_{l}^{j}+\Sigma \omega^{l^{\prime}} \wedge \omega_{l^{\prime}}^{j}+Q^{j}, \\
& d \omega^{j^{\prime}}=\Sigma \omega^{l^{\prime}} \wedge \omega_{l^{\prime}}^{j^{\prime}}+\Sigma \omega^{l} \wedge \omega_{l}^{j^{\prime}}+\mathbf{Q}^{j^{\prime}},
\end{aligned} \quad(l, j=1, \ldots, n),
$$

où les formes $\Omega^{j}$ et $\Omega^{j \prime}$ définissent la torsion.

Nous allons montrer que l'on peut imposer de manière canonique un tenseur de torsion mais la connexion affine correspondante n' est pas déterminée par les formules (19.3).

En raison de ces relations (19.3), on a : $d \Omega=\Sigma\left(\Omega^{j} \wedge \omega^{j^{\prime}}-Q^{j \prime} \wedge \omega^{f}\right)$; or la forme $d \Omega$ peut $s^{\prime}$ écrire: $d \Omega=\Sigma B_{\alpha \beta \gamma} \omega^{x} \wedge \omega^{\beta} \wedge \omega r$, les coefficients $B_{\alpha \beta \gamma}$ vérifiant les relations :

$$
B_{\alpha \beta \gamma}+B_{\alpha \gamma \beta}=0, \quad B_{\alpha \beta \gamma}=B_{\beta \gamma \alpha}=B_{\gamma \alpha \beta} \quad(\alpha, \beta, \gamma=1, \ldots, n) .
$$

Nous déterminerons les formes de torsion par les conditions:

$$
\Omega^{j}=\Sigma B_{\alpha \beta j} \omega^{\alpha} \wedge \omega^{\beta}, \quad Q^{j^{\prime}}=-\Sigma B_{\alpha \beta j} \omega^{\alpha} \wedge \omega^{\beta} \quad(j=1, \ldots, 2 n),
$$

conditions équivalentes à :

$$
\left.\Omega^{\beta}=\frac{1}{3} \alpha^{-1}\left(\omega^{\beta}\right)\right\rfloor d \Omega \quad(\beta=1, \ldots, 2 n)
$$

où $\alpha^{-1}$ est l'opérateur défini dans $\S 16$. On vérifie en effet que les former $\Omega^{\beta}$ ainsi definies soit les composantes $d$ 'une forme différentielle extérieure tensorielle : aux formes $\tilde{\omega r}=\Sigma u_{\beta}^{\top} \omega^{\beta}$ sont associées les formas

$$
\begin{aligned}
& \tilde{\mathbf{g}} \gamma=\frac{1}{3} \alpha^{-1}(\tilde{\omega} \Upsilon) \perp d \Omega, \quad \text { où }: \quad \tilde{\Omega} \gamma=\frac{1}{3} \alpha^{-1}\left(\Sigma u_{\beta}^{\gamma} \omega^{\beta}\right) \perp d \Omega \\
& \left.\left.=\frac{1}{3}\left[\Sigma u_{\beta}^{\gamma} \alpha^{-1}\left(\omega^{\beta}\right)\right]\right\rfloor d Q=\Sigma u_{\beta}^{\gamma}\left[\frac{1}{3} \alpha^{-1}\left(\omega^{\beta}\right)\right\rfloor d \Omega\right]=\Sigma u_{\beta}^{\gamma} \Omega^{\beta} .
\end{aligned}
$$


Pour que la structure soit intégrable e' est-à-dire pour que dans le voisinage $U$ il existe un système de coordonnées locales $x^{1}, \ldots, x^{n}, y^{4}, \ldots, y^{n}$ tel que la structure puisse être déterminée par les formes $\tilde{\omega}^{k}=\dot{d} x^{k}$, $\tilde{\omega}^{k^{\prime}}=d y^{k}$, il faut et il suffit que la torsion soit nulle; en effet si la structure est inté. grable, $\Omega$ peut s'écrire localement $d x^{1} \wedge d y^{\prime}+\ldots+d x^{n} \wedge d y^{n}$, donc $d \Omega=0$ et $\Omega^{x}=0$; inversement si la torsion est nulle, $d \Omega=0$ et $\Omega$ peut se mettre sous la forme canonique (18.2). Le pseudogroupe des automorphismes locaux de la structure symplectique est un pseudogroupe de LIE de type infini, ce qui est bien conforme au théorème 6.2 (puisque le choix de la torsion ne détermine pas canoniquement une connexion affine associée).

Nous allons introduire une torsion conforme, multipliée par un scalaire lorsqu'on multiplie la forme $Q$ par une fonction différentiable $\lambda$. Si la structure presque symplectique $\mathfrak{s}$ associée à la forme $Q$ est déterminée dans le voisinage $U$ d'un point de $V_{2 n}$ par les formes $\omega^{j}$, $\omega^{j}$, la structure $s_{\lambda}$ associée à la forme $\lambda \Omega$ peut être déterminée par les formes $\tilde{\omega}^{j}=\rho \omega^{j}, \tilde{\omega}^{j^{\prime}}=\rho \omega^{j^{\prime}}$ (où $\rho^{2}=\lambda$ ), si $\lambda$ est positif, par les formes $\tilde{\omega}^{j}=\rho \omega^{j}, \tilde{\omega}^{y}=-\rho \omega^{j \prime}$ (où $\rho^{2}=-\lambda$ ) si $\lambda$ est négatif. On a :

$$
\begin{aligned}
& d \tilde{\omega}^{j}=\Sigma \tilde{\omega}^{x} \wedge \omega_{\alpha}^{j}+\frac{d \rho}{\rho} \wedge \omega^{j}+\rho Q^{j}, \\
& d \tilde{\omega}^{j \prime}=\Sigma \tilde{\omega}^{x} \wedge \omega_{\alpha}^{j^{\prime}}+\frac{d \rho}{\rho} \wedge \omega^{j^{\prime}}+\varepsilon \rho \Omega^{\prime},
\end{aligned}
$$

Soient $\tilde{\Omega}^{j}, \tilde{\mathbf{\Omega}}^{j \prime}$ les formes de torsion de la structure $\mathfrak{s}_{\lambda}$. Si l'on pose: $\frac{d \rho}{\rho}=\Sigma\left(a_{k} \omega^{k}+a_{k^{\prime}} \omega^{k^{\prime}}\right)$, un calcul simple montre que l'on a :

$$
\begin{gathered}
\tilde{\Omega}^{j}=\rho\left[\Omega^{j}+2 \frac{d \rho}{\rho} \wedge \omega^{j}-a_{j^{\prime}} \omega^{j^{\prime}} \wedge \omega^{j}-a_{j^{\prime}} \Sigma \omega^{k} \wedge \omega^{k^{\prime}}\right] \\
\tilde{\Omega}^{j^{\prime}}=\varepsilon_{\rho}\left[\Omega^{y^{\prime}}+2 \frac{d \rho}{\rho} \wedge \omega^{j^{\prime}}-a_{j^{\prime}} \omega^{j} \wedge \omega^{j^{\prime}}-a_{j} \Sigma \omega^{k \prime} \wedge \omega^{k l}\right] .
\end{gathered}
$$

Par suite on définit la torsion conforme par les formes:

$$
\begin{aligned}
& \left.\Pi^{j}=\Omega^{j}-\frac{2}{n-1}\left[\Sigma B_{k j j^{\prime}} \omega^{k}+B_{k^{\prime} j j^{\prime}} \omega^{k^{\prime}}\right) \wedge \omega^{j}+\Sigma B_{k k^{\prime} j} \omega^{k} \wedge \omega^{k \prime}\right] \\
& \Pi^{j^{\prime}}=\Omega^{j^{\prime}}+\frac{2}{n-1}\left[\Sigma\left(B_{k j^{\prime} j} \omega^{k}+B_{k^{\prime} j^{\prime} j^{\prime}} \omega^{k \prime}\right) \wedge \omega^{j^{\prime}}+\Sigma B_{k^{\prime} k j \omega^{k \prime}} \wedge \omega^{k}\right] .
\end{aligned}
$$

La forme $\Sigma\left(\Pi^{j} \wedge \omega^{j^{\prime}}-\Pi^{j^{\prime}} \wedge \omega^{j}\right)$ est égale à $d \mathbf{Q}-\frac{6}{n-1}\left[\Sigma\left(B_{k j j^{\prime} \omega^{k}}+B_{k^{\prime} j^{\prime}} \omega^{k^{\prime}}\right) \wedge \mathbf{Q}\right]$ c' est-à-dire à : $d \Omega-\frac{1}{n-1} \tilde{\delta} \Omega \wedge \Omega=\psi$.

C'est pourquoi les formes $\delta \Omega$ et $\psi$ ont été appelees respectivement forme de torsion et forme de torsion conforme (cf. $\S 17$ ). 
Le theorème 18.1 peut s'exprimer de la façon suivante:

THÉnìm 19.1. - Etant donnée une structure presque symplectique $\mathfrak{B}$, pour $q u$ 'il existe une fonction $\lambda$ telle que la structure $\boldsymbol{s}_{\lambda}$ soit intégrable, il faut et pour $n>2$, il suffit que la torsion conforme soit nulle.

\section{Remarques concernant les structures symplectiques.}

Certains des résultats de ce paragraphe ont été également démontrés par GUGGENHEIMER [31].

Lorsque $d \Omega=0$, on a alors : $\delta \Omega=0$ et $\Omega$ est harmonique. Les relations suivantes sont également vérifiées:

$$
d L=L d, \quad \Lambda \tilde{\delta}=\tilde{\delta} \Lambda .
$$

Si la forme $\psi_{p}$ est effective, on a: $\tilde{*} \psi_{p} \wedge \Omega=0, d \tilde{*} \psi_{p} \wedge \Omega=0$, ou : $\tilde{*} \tilde{\delta} \psi_{p} \wedge \Omega=0$. Donc si $\psi_{p}$ est effective, $\tilde{\delta} \psi_{p}$ est également effective; d' où : $d \tilde{*} \psi_{p}=\approx \tilde{\delta} \psi_{p}=$ $=\varepsilon_{p-s} \tilde{\delta} \psi_{p} \wedge \frac{\Omega^{n-p-1}}{(n-p+1) !}=\varepsilon_{p} d \psi_{p} \wedge \frac{\Omega^{n-p}}{(n-p) !}$ puisque $* \psi_{p}=\varepsilon_{p} \psi_{p} \wedge \frac{\Omega^{n-p}}{(n-p) !}$. Donc: $\left[d \psi_{p}-(-1)^{p-1} \frac{\tilde{\delta} \psi_{p} \wedge \Omega}{n-p+1}\right] \wedge \Omega^{n-p}=0$, soit en raison de (15.17):

$$
d \psi_{p}=\psi_{p+1}+(-1)^{p-1} \frac{\tilde{\delta} \psi_{p} \wedge \Omega}{n-p+1}
$$

où $\Psi_{p+1}$ est effective. D' où :

THÉم亡m 20.1. - Si la forme $\psi_{p}$ est effective, sa différentielle $d \psi_{p}$ est la somme directe $d$ 'une forme effective et d'une forme de classe 1. Pour que $d \psi_{p}$ soit effective, il faut et il suffit que $\tilde{\delta} \psi_{p}=0$. (On a alors $\left.\tilde{\delta} d \psi_{p}=0\right)$.

Corollarre 20.1. - Si $\varphi_{p}$ est une forme simple de classe $k$, d $\varphi_{p}$ est la somme directe $d^{\prime}$ une forme de classe $k$ et $d$ 'une forme de classe $k+1, \tilde{\delta} \varphi_{p}$ la somme directe d'une forme de classe $k-1$ et d'une forme de classe $k$. En particulier si $d \varphi_{p}$ est simple, $\tilde{\delta} \varphi_{p}$ est simple également.

En effet, soit : $\varphi_{p}=\psi_{p-2 k} \wedge \frac{\Omega^{k}}{k !}$; on a :

$$
\begin{gathered}
d \varphi_{p}=d \psi_{p-2 k} \wedge \frac{\Omega^{k}}{k !}, \\
\tilde{*} \tilde{\delta} \varphi_{p}=d \tilde{*} \varphi_{p}=\varepsilon_{p-2 k} d \psi_{p-2 k} \wedge \frac{\Omega^{n-p-k}}{(n-p+k) !}=\varepsilon_{p-2 k} \frac{k !}{(n-p+k) !} d \varphi_{p} \wedge \Omega^{n-p} ;
\end{gathered}
$$

pour que $d \varphi_{p}$ soit simple, il faut et il suffit que $d \psi_{p-q k}$ le soit; il en est alors de mème de $\tilde{\delta} \varphi_{p}$ et réciproquement.

COROLLAIRE 20.2. - Une forme simple fermée est harmonique par rapport $\grave{a} \Omega$. Ce corollaire résulte de la formule (20.4).

Inversement si $\tilde{\delta} \varphi_{p}=0, d \psi_{p-2 h} \wedge \Omega^{n-p+k}=0$, ce qui entraine $d \varphi_{p}=0$ si $k \geq 1$. Si $k=0, \tilde{\delta} \varphi_{p}=0$, entraine seulement que $d \varphi_{p}$ est effective. 
THénime 20.2. - Si une forme $\varphi_{p}$ est harmonique par rapport $\dot{a} \Omega$, ses composantes simples sont harmoniques également.

La démonstration utilise la décomposition en formes simples de $\varphi_{p}$ et $\tilde{*} \varphi_{p}$ ainsi que le thérème 20.1. Les composantes simples de $d \varphi_{p}$ et $d \tilde{*} \varphi_{p}$ étant nulles, on démontre successivement que les compósantes de classe $0,1, \ldots, q$ de $\varphi_{p}$ sont fermées, done harmoniques.

On déduit da théorème 20.2 la proposition: si $\varphi_{p}$ est harmonique par rapport à $\Omega$, il en est de même de $\varphi_{p} \wedge \Omega^{k}$.

REMARQUE: de la relation (20.2) on déduit :

$$
\tilde{\delta}\left(\psi_{p} \wedge \Omega^{k}\right)=(-1)^{k} k \psi_{p+1} \wedge \Omega^{k+1}+\frac{n-p+k+1}{n-p+1} \tilde{\delta} \psi_{p} \wedge \Omega^{k}
$$

d'où :

$\tilde{\delta}\left(\psi_{p} \wedge \Omega^{k}\right)-\left[\tilde{\delta}\left(\psi_{p} \wedge \Omega^{k}\right)\right] \wedge \Omega=(-1)^{p} \psi_{p+1} \wedge \Omega^{k}-\frac{\tilde{\delta} \psi_{p} \wedge \Omega^{k+1}}{n-p+1}=(-1)^{p} d\left(\psi_{p} \wedge \Omega^{k}\right)$

Donc si $\varphi_{p}$ est une forme quelconque:

$$
\tilde{\delta} L \varphi_{p}-L \tilde{\delta} \varphi_{p}=(-1)^{p} d \varphi_{p} .
$$

On démontre de même:

$$
d \Lambda \varphi_{p}-\Lambda d \varphi_{p}=(-1)^{p \tilde{\delta} \varphi_{p}} .
$$

Si l'on supposait la forme $\Omega$ cofermée mais non fermée les relations démontrées dans ce paragraphe ne sont plus applicables à une forme de degré quelconque mais certains résultats subsistent pour les formes de degré 1 . Soit $\omega$ une forme de PFAFF; comme $d \tilde{*} \Omega=0$, on $a: \tilde{*} \tilde{\delta} \omega=d \tilde{*} \omega=d(\omega \wedge \tilde{*} \Omega)=$ $=d \omega \wedge \tilde{*} \Omega=(\tilde{*} d \omega) \wedge \Omega$ (en raison de $(15.19)) ;$ d'où :

$$
\tilde{\delta} \omega=\Lambda d \omega \text {. }
$$

La formule (20.6) est donc encore applicable aux formes de PFaff. Pour que la forme $\omega$ soit cofermée, il faut et il suffit que $d \omega$ soit effective. En particulier une forme de PFaff fermée est harmonique par rapport a $\Omega$.

\section{Chapitre V.}

\section{Sur les variétés presque complexes et presque paracomplexes.}

A) Structures presque complexes et presque paracomplexes.

21. Détermination de ces structures.

Sur une variété $V_{z n}$, de dimension $2 n, r$ fois différentiable $(r \geq 3)$ une structure infinitésimale régulière (cf. $\$ 5$ ), de groupe structural $L_{n}^{\prime}$ (groupe linéaire homogène complexe) est une structure presque complexe [24].

Nous désignerons par presque paracomplexe [41] une structure infinitésimale régulière dont le groupe structural est le groupe linéaire homogène paracomplexe (\$ 11). 
Soit sur une variété $V_{2 n}$ une structure presque complexe $T\left(V_{2 n}, C^{n}, L^{\prime}, H\right)$ : l'ensemble des homéomorphismes $h_{x}$ de $R^{2 n}$ (identifié à $C^{n}$ ) sur l'espace tangent $T_{x}$ à $V_{2 n}$ en $x \in V_{z n}$ détermine dans $T_{x}$ une structure d'espace vectoriel complexe. Ou peut définir l'espace fibré $T^{c}\left(V_{2 n}\right)$, associé à $T\left(V_{2 n}\right)$, dont les fibres $T_{x}{ }^{c}$ sont isomorphes à $C^{2 n}$; tout sous-espace de $T_{x}{ }^{c}$ s'ap- $^{2}$ pelle $n$-élément de contact complexe tangent en $x$. Une structure presque complexce peut être déterminée par la donnée d'un champ d'automorphismes $\mathfrak{J}_{x}$. de l'espace tangent $T_{x}$, tels que $\left(\mathfrak{J}_{x}\right)^{2}=-1$ ou par la donnée d'un champ $C$ de n-éléments de contact complexes $X_{n}$ tels qu'en chaque point $X_{n}$ et $\bar{X}_{n}$ soient supplémentaires (of. $\S 10$ ).

De même si l'on se donne sur une variété $V_{2 n}$ une structure presque paracomplexe, l'ensemble des homéomorphismes de $R^{2 n}$ (muni de sa structure de module unitaire paracomplexe) sur l'espace tangent $T_{x}$ définit dans $T_{\infty}$ une structure de module unitaire paracomplexe (cf. \& 2). Donc une structure presque paracomplexe peut être déterminée par la donnée d'un champ de $(n, n)$-involutions $\mathscr{F}_{x}$ de l'espace tangent $T_{x}$, ou encore par la donnée de deux champs $C_{1}$ et $C_{2}$ deux fois différentiables de n-éléments de contact supplémen. taires $X_{n}$ et $X_{n}^{\prime}$.

Le problème d'existence d'une structure presque complexe ou presque paracomplexe conduit à des uobstacles». (Pour le cas presque complexe voir [24]).

Dans la suite de ce chapitre nous adopterons les conventions suivantes (sauf mention spéciale): les indices latins varient de 1 à $n$, les indices grees de 1 à $2 n$, arec: $s^{\prime}=s+n ; \alpha^{\prime}=\alpha+n$ si $\alpha \leq n, \alpha^{\prime}=\alpha-n$ si $\alpha>n$.

Dans le voisinage $U$ d'un point de $V_{2 n}$ une structure presque complexe pent être déterminée par la donnée de $n$ formes de PFaff différentiables complexes linéairement indépendantes dans le domaine complexe $\omega^{8}=\alpha^{8}+i \alpha^{8^{\prime}}$ (où les $2 n$ formes réelles $\alpha^{s}$, $\alpha^{s}$ sont linéairement indépendantes); la même structure peut encore être déterminée par les $n$ formes de Pfaff $\tilde{\omega}^{l}=\Sigma u_{8}^{l} \omega^{8}$ où les $u_{s}^{l}$ sont des fonctions locales différentiables à valeurs complexes, definissant en chaque point une transformations de $L_{n}^{\prime}$.

De même dans un voisinage $U$, une structure presque paracomplexe pent être déterminée par la donnée de $n$ formes paracomplexes $\varphi^{s}=\alpha^{s}+j \alpha^{s t}$ (les $2 n$ formes $\alpha^{z}, \alpha^{s f}$ étant linéairement indépendantes) ou par la donnée de $n$ autres formes paracomplexes $\tilde{\varphi}^{l}=\Sigma p_{s}{ }^{l} \varphi^{s}$ où les $p_{s}^{l}=a_{s}{ }^{l}+j b_{s}{ }^{l}$ sont des fonctions locales à valeurs paracomplexes définissant en chaque point une transformation de $\stackrel{o}{L}_{2 l}^{\prime}$ (on suppose que le fonctions $a_{8}{ }^{l}$ et $b_{8}{ }^{l}$ sont différentiables). Les deux champs $C_{1}$ et $C_{2}$ sont définis dans le voisinage $U$ par équations :

$$
\omega^{s}=\alpha^{s}-\alpha^{8 \prime}=0, \quad \omega^{8 \prime}=\alpha^{s}+\alpha^{8 \prime}=0 \quad(s=1, \ldots, n)
$$

ou en posant $\tilde{\omega}^{l \prime}=\Sigma u_{s}{ }^{\prime} \omega^{\prime}, \tilde{\omega}^{l}=\Sigma u_{s}{ }^{l} \omega^{s} \quad\left(\operatorname{avec} u_{s}{ }^{l}=a_{s}{ }^{l}-b_{s}{ }^{l}, u_{s}{ }^{l}=a_{s}{ }^{l}+b_{s}{ }^{l}\right)$ par les équations : $\tilde{\omega}^{\prime \prime}=0, \tilde{\omega}^{l}=0$. 
Aux formes $\omega^{8}, \omega^{s \prime}$ correspond en chaque point une base duale de $T_{x}$ telle que le vecteurs $e_{s}$ soient les vectenrs propres de $\mathfrak{F}_{x}$ corlespondant à +1 , les vecteurs $e_{s^{\prime}}$ les vecteurs propres correspondant à -1 .

En résumé une structure presque complexe (resp. paracomplexe) peut être déterminée dans le voisinage $U$ d'un point de $V_{z n}$ par $2 n$ formes de PFAfF linéairement indépendantes dans le domaine réel $\omega^{*}$, $\omega^{s^{\prime}}$; si la structure est presque complexe, les $\omega^{s}$ et $\omega^{\varepsilon^{\prime}}$ sont complexes et liées par les relations : $\omega^{8 \prime}=\omega^{8}(s=1, \ldots, n)$; si la structure est presque paracomplexe les formes $\omega^{s}$ et $\omega^{s \prime}$ sont réelles. Dans ce qui suit le composantes $T_{\beta \gamma}^{\alpha \ldots . . .}$ d'nn tenseur associé à une structure presque paracomplexe sont réelles, celles d'un tenseur associé à une structure presque complexe sont complexes (en général), liées par les relations $T_{\beta^{\prime} \gamma^{\prime} \ldots . . .}^{\alpha^{\prime}}=\bar{T}_{\beta \gamma \ldots}^{\alpha \ldots}$; cette convention s'appliquera également aux composantes d'une forme différentielle extérieure tensorielle (par exemple courbure et torsion) ainsi qu'aux formes $\omega_{s}^{l}$ (qui ne constituent pas les composantes d' un tenseur).

Une connexion affine (cf. \& b) associèe à la structure presque complexe (resp. presque paracomplexe) est definie par l'ensemble des formes $\omega^{s}$, $\omega^{s \prime}$ et de $2 n^{2}$ formes $\omega_{l}^{s}, \omega_{l^{\prime}}{ }^{s^{\prime}}$ linéaires en $\omega^{t}$ et $\omega^{t^{\prime}}$; les équations $(6.11)^{\prime}$ peuvent alors s' écrire :

$$
\begin{aligned}
& d \omega^{\varepsilon}=\Sigma \omega^{l} \wedge \omega_{l}^{t}+\Gamma^{s}, \\
& d \omega^{s^{\prime}}=\Sigma \omega^{l^{\prime}} \wedge \omega_{l^{\prime}}^{t^{\prime}}+\Gamma^{s^{\prime}},
\end{aligned} \quad(s=1, \ldots, n) .
$$

où l'ensemble des formes $\Gamma^{s}$ et $\Gamma^{s \prime}$ définit la torsion. On peut déterminer canoniquement la torsion par les conditions:

$$
\begin{aligned}
& \Gamma^{s}=\Sigma A_{l t}^{s} \omega^{t^{\prime}} \wedge \omega^{l^{\prime}}, \\
& \Gamma^{s \prime}=\Sigma A_{l t^{\prime}, \omega^{t}}^{s} \wedge \omega^{t}, \\
& (s=1, \ldots, n) .
\end{aligned}
$$

On vérifie que ces conditions permettent de définir les composantes d'une forme différentielle extérieure tensorielle; en effet si l'on associe aux formes $\tilde{\omega}^{l}=\Sigma u_{s}{ }^{2} \omega^{s}, \tilde{\omega}^{\prime \prime}=\Sigma u_{s^{\prime}}{ }^{\prime \prime} \omega^{s^{\prime \prime}}$ les formes $\tilde{\Gamma}^{l}=\Sigma u_{s}{ }^{l} \Gamma^{s}, \tilde{\Gamma}^{l^{\prime}}=\Sigma u_{s^{\prime}}{ }^{\prime \prime} \Gamma^{s^{\prime}}$, ces formes peuvent s' écrire: $\tilde{\Gamma}^{l}=\Sigma \tilde{A}_{h u}^{l} \tilde{\omega}^{h^{\prime}} \wedge \tilde{\omega}^{u^{\prime}}, \tilde{\Gamma}^{l^{\prime}}=\Sigma \tilde{A}_{h^{\prime} w^{\prime}}^{\omega^{\prime}} \tilde{\omega}^{h} \wedge \tilde{\omega}^{u}$.

En tenant compte des conditions (21.3) les formes $\omega_{l}^{s}$ sont définies mod. $\omega^{l}$ et les formes $\omega_{l^{\prime}}^{s^{\prime}}$ sont définies mod. $\omega^{t^{\prime}}$ : ces condition ne suffisent pas pour déterminer canoniquement une connexion affine.

Dans le cas d'une structure presque paracomplexe, si l'un des champs seulement $\left(C_{1}\right.$ par exemple) est complètement intégrable, on a: $\Gamma^{8 \prime}=0, \Gamma^{*} \neq 0$.

Pour qu'une structure presque complexe soit intégrable, e'est-à-dire puisse être définie dans le voisinage $U$ d'un point quelconque de $V_{q n}$ par $n$ formes complexes linéairement indépendantes $\tilde{\omega}^{1}=d z^{1}, \ldots, \tilde{\omega}^{n}=d z^{n}$, il fant que la torsion soit nulle (propriété démontrée par G. DE RHAM); C. EHREsmanN a démontré que cette condition est suffisante lorsqu'on suppose la structure déterminée par $n$ formes $\omega^{8}=\alpha^{8}+i \alpha^{8 \prime}$ telles que les formes de FFarF 
réelles $\alpha^{8}, \alpha^{8 \prime}$ soient analytiques réelles. Cette condition réalisée, la structure presque complexe dérive d' une structure analytique complexe.

Une structure presque paracomplexe est intégrable s'il existe dans le voisinage d'un point quelconque de $V_{2 n}$ un système de coordonnés locales $x^{1}, \ldots, x^{n}, y^{4}, \ldots, y^{n}$ tel que la structure puisse être déterminée par les $n$ formes de PFAFr paracomplexes $\tilde{\varphi}^{h}=d x^{h}+j d y^{h}$; le champs $C_{1}$ et $C_{2}$ sont alors definis respectivement par les équations: $d x^{h}-d y^{h}=0, d x^{h}+d y^{h}=0$ $(h=1, \ldots, n)$; les champs $C_{1}$ et $C_{2}$ sont par conséquent complètement intégrables. Dono en raison da théorème de FroBeniUs, pour qu'une structure presque paracomplexe soit intégrable, il faut et il suffit que sa torsion soil nulle. Si cette condition est réalisée, Ja structure sera dite paracomplexe: une variété paracomplexe $V_{2 n}$ est une variété sur laquelle sont définis deux champs deux fois différentiables de n-éléments de contact complètement intégrables supplémentaires. Chacun de ces champs définit sur $V_{z n}$ une structure feuilletée différentiable.

Le pseudogroupe des automorphismes locaux d'une structure presque complexe ou presque paracomplexe est un pseudogroupe de LIE.

B) Structures presque hermitiennes et presque parahermitiennes.

22. Connexion hermitienne et connexion parahermitienne.

Une structure presque hermitienne (resp. parahermitienne) est par définition une structure subordonnée à une structure presque complexe (resp. paracomplexe) dont le groupe structural est le groupe unitaire $U_{n}$ (resp. paraunitaire $\dot{U}_{n}$ ). Les structures presque hermitiennes (ainsi que les structures presque complexes) ont été définies par C. Ehresmann [24]. L'étude des structures presque parahermitiennes a été faite dans [41].

Si la structure presque complexe à laquelle est subordonnée la structure presque hermitienne dérive d'une structure complexe, la structure considérée est dite hermitienne; de même une structure presque parahermitienne subordonnée à une structure paracomplexe (c'est-à-dire telle que les champs $C_{4}$ et $C_{2}$ soient complètement intégrables), est dite parahermitienne.

En raison des propriétés énoncées dans § 14, une structure presque hermitienne peut être définie par la donnée d'une métrique riemannienne $F$ définie positive et d'un champ d'automorphismes $\mathfrak{J}_{x}$ de l'espace tangent $T_{x}$ à $V_{2 n}$ en $x, \mathfrak{J}_{x}$ étant de carré -1 et laissant invariante la restriction $F_{x}$ de $F$ a $T_{x}$. Cette structure peut encore être déterminée par la donnée d'une métrique riemannienne $F$ et d'une forme différentielle extérieure quadratique $\Omega$ de rang $2 n$ en tout point de $V_{2 n}$ et échangeable avec $F$.

De même une structure presque hermitienne d'espèce $k$ (de groupe structural le groupe unitaire d'espèce $k$ ) peut être déterminée par la donnée d'une métrique riemannienne $F^{\prime k\rangle}$ (décomposable en chaque point en une somme de $2 k$ carrés positifs et $2 n-2 k$ carrés négatifs) et d'un champ d'automor- 
phismes $\mathfrak{I}_{x}$ laissant invariante $F_{x}^{(k)}$ ou par la donnée de $F^{\prime k\rangle}$ et d'une forme différentielle extérieure quadratique échangeable avec $F^{(k)}$.

Une structure presque parahermitienne peut être déterminée par la donnée de deux champs $C_{1}$ et $C_{2}$ deux fois différentiables de $n$-élémentes de n-éléments de contact supplémentaires $X_{n}$ et. $X_{n}^{\prime}$ et d'une forme différentielle extérieure quadratique $\Omega$ de rang $2 n$, telle que $X_{n}$ et $X_{n}^{\prime}$ en soient des éléments intégraux, ou bien par la donnée des champs $C_{1}$ et $C_{2}$ et d'une métriqne riemannienne indéfinie $F^{\prime}$ dont $X_{n}$ et $X_{n}^{\prime}$ sont des eléments intégraux.

Une structure presque hermitienne (resp. presque hermitienne d'espèce $k$, presque parahermitienne) est donc subordonné à une structure symplectique et à une structure riemannienne dont la métrique est définie positive (resp. indéfinie). Lorsqu'elle dérive d'une structure symplectique (c'est-à-dire si $d \boldsymbol{Q}=0$ ) la structure est dite presque kahlérienne (resp. presque kühlérienne d'espèce $k$, presque parakählérienne); une structure presque kăhlérienne (resp. presque kăhlérienne d'espèce $k$ ) telle que la structure presque complexe dérive d'une structure complexe est dite kăhlérienne (resp. kăhlérienne d'espèce $k$ ). Définition analogue d'une structure parakählórienne.

Dans le voisinage $U$ d'un point de $V_{2 n}$ une structure presque hermitienne peut ètre déterminée par la donnée de $2 n$ formes de Pfaff complexes $\omega^{s}$, $\omega^{s \prime}$ telles que : $\omega^{s \prime}=\bar{\omega}^{s}$, la forme $d^{\prime}$ Hermite et la forme extérieure associées à la structure pouvant s'écrire :

$$
\Phi=\Sigma \omega^{s} \omega^{s^{\prime}}, \quad \Omega=i \Sigma \omega^{8} \wedge \omega^{8 \prime} .
$$

De meme une structure presque parahermitienne peut être déterminée localement par la donnée de $2 n$ formes de PFafF réelles $\omega^{8}$, $\omega^{\prime \prime}$, linéairement indépendantes telles que la métrique riemannienne et la forme extérieure associées à la structure puissent s'écrire:

$$
F^{\prime}=\Sigma \omega^{s} \omega^{s \prime}, \quad \Omega=\Sigma \omega^{s} \wedge \omega^{s t} .
$$

La structure presque hermitienne (resp. parahermitienne) peut encore etre déterminée par les $2 n$ formes $\tilde{\omega}^{l}=\Sigma u_{s}^{l} \omega^{s}, \tilde{\omega}^{l^{\prime}}=\Sigma u_{s^{\prime}}^{l^{\prime}} \omega^{s^{\prime}}$ les $u_{s}^{l}$ et $u_{s^{\prime}}^{l^{\prime}}$, définissant en chaque point une transformation du groupe unitaire $U_{n}$ (resp. paraunitaire $\dot{U}_{n}$ ). On a: $\Sigma u_{s}^{l} u_{t^{\prime}}^{\prime}=\delta_{t}^{s}$. La restriction à l' espace tangent $T_{x}$ des $2 n$ formes $\tilde{\omega}^{l}, \tilde{\omega}^{l \prime}$ constitue un "corepère unitaire» (resp. para-unitaire).

On pent déterminer de manière unique des formes $\omega_{l}^{g}$, $\omega_{l \prime}^{8 \prime}$ linéaires en $\omega^{l}$ et $\omega^{l^{\prime \prime}}$ telles que :

$$
\begin{aligned}
& d \omega^{s}=\Sigma \omega^{l} \wedge \omega_{l}^{s}+\Sigma A_{l t}^{s} \omega^{l} \wedge \omega^{t}+\Sigma B_{l t}^{s} \omega^{l^{\prime}} \wedge \omega^{t^{\prime}}, \\
& d \omega^{z^{\prime}}=\Sigma \omega^{l^{\prime \prime}} \wedge \omega_{l^{\prime}}^{s^{\prime}}+\Sigma A_{l^{\prime} t^{\prime}}^{s^{\prime}}\left(\omega^{\prime \prime} \wedge \omega^{t^{\prime}}+\Sigma B_{l^{\prime} t^{\prime} \omega^{l}}^{s^{\prime}} \wedge \omega^{t},\right. \\
& \omega_{l}^{s}+\omega_{s^{\prime}}^{l^{\prime}}=0, \quad A_{\beta \gamma}^{\alpha}+A_{\gamma \beta}^{\alpha}=0, \quad B_{\beta \gamma}^{\alpha}+B_{\gamma \beta}^{\alpha}=0, \quad(\alpha, \beta, \gamma=1, \ldots, 2 n),
\end{aligned}
$$

les convention adoptées étant toujours celles indiquées dans \$ 21. 
Ces équations ont été indiquées par CHERN [18] dans le cas hermitien (pour le cas presque hermitien voir [41]).

On peut ainsi associer canoniquement d̀ la structure presque hermitienne (resp. presque parahermitienne) une connexion affine, en plus de la connexion riemannienne associée à la métrique $F$ (resp. $F^{\prime}$ ). Cette connexion sera appelée hermitienne (resp. parahermitienne).

Les formes $\Omega^{s}=\Sigma A_{l t}^{s} \omega^{l} \wedge \omega^{t}, \Omega^{s \prime}=\Sigma A_{l t^{\prime}}^{s^{\prime}} \omega^{l^{\prime}} \wedge \omega^{t \prime}$ définissent la première torsion de la connexion hermitienne (resp. parahermitienne) la deuxième tor. sion étant définie par les formes $\Gamma^{s}=\Sigma B_{l t}^{s} \omega^{l^{\prime}} \wedge \omega^{t^{\prime}}, \Gamma^{s^{\prime}}=\Sigma B_{l^{\prime} t^{\prime}}^{s^{\prime}} \omega^{l} \wedge \omega^{t}$.

La courbure est définie par les formes $\Omega_{l}^{s}=d \omega_{l}^{s}-\Sigma \omega_{l}^{t} \wedge \omega_{t}^{s}$ et $\mathbf{Q}_{l^{\prime}}^{s^{\prime}}=d \omega_{l^{\prime}}^{s^{\prime}}-\Sigma \omega_{l^{\prime}}^{t^{\prime}} \wedge \omega_{t^{\prime}}^{s^{\prime}}$ qui vérifient les équations: $\mathbf{Q}_{l}^{s}+\mathbf{Q}_{s^{\prime}}^{l^{\prime}}=0$, et les relations généralisant les identités de BIANCHI (of. formules (6.14) et $(6.15$ ]

$$
\begin{aligned}
& d\left(\Omega^{s}+\Gamma^{s}\right)+\Sigma\left(\Omega^{l}+\Gamma^{l}\right) \wedge \omega_{l}^{s}-\Sigma \omega^{l} \wedge \Omega_{l}^{s}=0, \\
& d\left(\Omega^{s^{\prime}}+\Gamma^{s^{\prime}}\right)+\Sigma\left(\Omega^{v^{\prime}}+\Gamma^{l^{\prime}}\right) \wedge \omega_{l^{\prime}}^{s^{\prime}}-\Sigma \omega^{l^{\prime}} \wedge \Omega_{l^{\prime}}^{s^{\prime}}=0, \quad(s, l=1, \ldots, n) . \\
& d S_{l}^{s}+\Sigma Q_{l}^{t} \wedge \omega_{t}^{s}-\Sigma \omega_{l}^{t} \wedge \Omega_{t}^{s}=0, \\
& d \Omega_{l^{\prime}}^{s^{\prime}}+\Sigma Q_{l^{\prime}}^{t^{\prime}} \wedge \omega_{t^{\prime}}^{s^{\prime}}-\Sigma \omega_{l^{\prime}}^{t^{\prime}} \wedge \Omega_{t^{\prime}}^{s^{\prime}}=0 .
\end{aligned}
$$

En raison des équations (22.3), on a :

$$
d \Omega=\Sigma\left(\Omega^{s}+\Gamma^{s}\right) \wedge \omega^{s \prime}-\Sigma\left(\Omega^{s \prime}+\Gamma^{s \prime}\right) \wedge \omega^{s} .
$$

En raison d'une remarque de $\$ 6$, pour que la connexion soit intégrable, il faut et il suffit que la courbure et la torsion soient nulles. Il existe alors dans le voisinage $U$ un système de coordonnées locales complexes (resp. paracomplexes) $z^{s}$ tel que la forme d'Hermite (resp. parahermitienne) puisse s'écrire: $\Sigma d z^{s} d \overline{z^{s}}$.

Si la structure est hermitienne (resp. parahermitienne) la deuxième torsion est nulle; si la structure est presque kählérienne (resp. presque parakählérienne), en raison de (22.5) la première torsion est nulle et $\Sigma\left(\Gamma^{s} \wedge \omega_{s}{ }^{\prime}-\right.$ $\left.-\Gamma^{s t} \wedge \omega^{s}\right)=0$, soit :

$$
B_{t s}^{l}+B_{s l}^{t}+B_{l t}^{s}=0, \quad B_{t^{\prime} s^{\prime}}^{v^{\prime}}+B_{s^{\prime} l^{\prime}}^{v^{\prime}}+B_{l^{\prime} t^{\prime}}^{s^{\prime}}=0 \quad(l, t, s=1, \ldots, n) .
$$

Les formes de courbure de la connexion hermitienne associée à une structure presque hermitienne (resp. presque parahermitienne) peuvent s'écrire:

$$
\begin{aligned}
& \mathbf{Q}_{l}^{s}=\Sigma\left(R_{t^{\prime} h^{\prime} \omega^{t^{\prime}}} \wedge \omega^{h}+R_{l t h \omega^{t}}^{s} \wedge \omega^{h}-R_{s t^{\prime} h^{\prime}\left(\omega^{t \prime}\right.}^{t^{\prime \prime}} \wedge \omega^{h^{\prime}}\right) \\
& \left.\left.\mathbf{Q}_{l^{\prime}}^{s \prime}=\Sigma\left(R_{l^{\prime} t h^{\prime}}^{s^{\prime}}\right)^{t} \wedge \omega^{h^{\prime}}+R_{l^{\prime} t^{\prime} h^{\prime}}^{s^{\prime}}\right)^{t^{\prime}} \wedge \omega^{h^{\prime}}-R_{s t h}^{l} \omega^{t} \wedge \omega^{h}\right)
\end{aligned}
$$

où :

$$
R_{l l^{\prime} h}^{s}=R_{s^{\prime} h t^{\prime}}^{l^{\prime}}, \quad R_{l t h}^{s}+R_{l h t}^{s}=0, \quad R_{l^{\prime} t^{\prime} h^{\prime}}^{s}+R_{l^{\prime} h^{\prime} t^{\prime}}^{s^{\prime}}=0 \quad(s, l, t, h=1, \ldots, n) .
$$

Dans le cas d'une structure presque parahermitienne, si l'un an moins des champs définissant la structure $\left(C_{\mathrm{t}}\right.$ par exemple) est complètement intégrable, on a: $B_{l t}^{s}=0,(s, l, t=1, \ldots, n)$. En utilisant les identités de BlavoH, 
on obtient: $R_{t h}^{s}=0,(s, l, t, h=1, \ldots, n)$; donc les formes induites par les formes $\Omega_{l}^{s}$ sur les variétés intégrales du champ $C_{1}$ (défini par $\omega^{* \prime}=0$ ) sont nulles. D' où :

Théonime 22.1. - Si au moins l'un des champs associés à une structure presque parahermitienne est complètement intégrable, la connexion induite sur les variétés intégrales de ce champ est à courbure nulle. Si, de plus, la première torsion de la connexion parahermitienne est nulle (ce qui a lieu notamment quand la structure est presque paraköhlérienne), la structure induite sur les variétés intégrales du champ est une structure intégrable.

Lorsque l'ensemble des formes définissant la deuxième torsion est nul, ce qui a lien pour les structures hermitiennes et parahermitiennes, on a alors : $R_{l t h}^{s}=0, R_{l^{\prime} t^{\prime} h^{\prime}}^{s^{\prime}}=0$, d'où :

$$
\Omega_{l}^{s}=\Sigma R_{t^{\prime} h^{\prime} \omega^{\prime \prime}} \wedge \omega^{h}, \quad \Omega_{\nu}^{s^{\prime}}=\Sigma R_{l t h^{\prime}}^{s^{\prime}} \wedge \omega^{h^{\prime}} \quad(s, l=1, \ldots, n),
$$

(relations démontrées par CHERN pour les structures hermitiennes). Dans le cas des structures parahermitiennes, en raison du théorème 22.1, la connexion induite sur les variétés intégrales de chacun des champs $C_{1}$ et $C_{2}$ est à courbure nulle.

Si la première torsion d'une structure presque hermitienne (resp. parahermitienne) est nulle, en atilisant encore les identités de BIarchr, on démontre les relations :

$$
R_{l t h}^{s}+R_{t h l}^{s}+R_{h t l}^{s}=0, \quad R_{v^{\prime} t^{\prime} h^{\prime}}^{s^{\prime}}+R_{t^{\prime} h \eta^{\prime}}^{s^{\prime}}+R_{h^{\prime} l^{\prime \prime}}^{s^{\prime}}=0 \quad(8, h, l, t=1, \ldots, n) .
$$

Une structure presque hermitienne d'espèce $k$ peut être déterminée dans un voisinage $U$ par la donnée de $2 n$ formes de Pfaff complexes $\omega^{*}$, $\omega^{* \prime}$ telles que $\omega^{\prime \prime}=\bar{\omega}^{s}$ si $s \leq k, \omega^{s \prime}=-\bar{\omega}^{s}$ si $s>k$, la forme d' HERMITE et la forme extérieure associées à la structure pouvant s' écrire :

$$
\begin{aligned}
& \Phi^{(k)}=\Sigma \omega^{s} \omega^{\prime \prime} \\
& \Omega=i \Sigma \omega^{s} \wedge \omega^{s \prime} .
\end{aligned}
$$

On peut définir un "corepère para-unitaire d'espèce $k$ ». Les formules (22.3), (22.4), (22.7) sont applicables aux structures presque hermitiennes d'espèce $k$, à condition d'adopter les conventions suivantes :

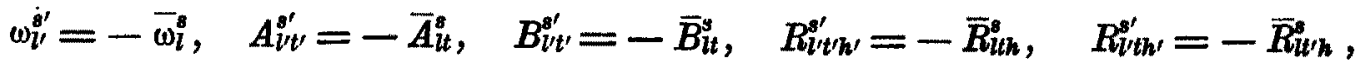
si le nombre d'indices supérieurs à $k$ est impair,

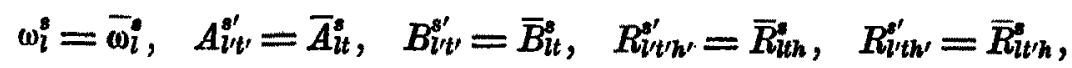

dans le cas contraire; on peut ainsi associer canoniquement une connexion affine à une structure presque hermitienne d'espèce $k$. 
23. Structures isotropes et localement homogènes [30] et [41].

Rappelons (\$ 5) qu' une structure presque hermitienne (resp. presque parahermitienne) est dite isotrope au point $x$ s'il existe un automorphisme de la structure transformant un corepère unitaire (resp. para-unitaire) d'origine $x$ en un corepère unitaire (resp. para-unitaire) arbitraire de même origine; la structure sera dite localement homogène dans le voisinage $U$ de $x$ s'il existe un automorphisme de la structure transformant un corepère d'origine $x$ en un corepère d'origine $x^{\prime} \& U$. Pour que la structure soit isotrope en $x$ (resp. localement homogène), il faut que les composantes des tenseurs de courbure et de torsion soient indépendantes du corepère considéré (resp. égales à des constantes). Les conventions adoptées sont celles de § 21.

Aux formes $\tilde{\omega}^{h}=\Sigma u_{s}^{h} \omega^{s}, \widetilde{\omega}^{h \prime}=\Sigma u_{s^{\prime}}^{h^{\prime}} \omega^{s \prime}$ sont associées les formes $\tilde{\Omega}^{h}, \tilde{\Omega}^{h^{\prime}}$, $\tilde{\Gamma}^{h}, \tilde{\Gamma}^{h^{\prime}}, \tilde{\Omega}_{m}^{h}, \tilde{\Omega}_{m^{\prime}}^{h^{\prime}}$, telles que (cf. $\S 6$ )

$$
\begin{array}{ll}
\tilde{\Omega}^{h}=\Sigma u_{s}^{h} \Omega^{s}, & \tilde{\Omega}^{h^{\prime}}=\Sigma u_{s^{\prime}}^{h^{\prime}} \Omega^{s^{\prime}}, \\
\tilde{\Gamma}^{h}=\Sigma u_{s}^{h} \Gamma^{s}, & \tilde{\Gamma}^{h^{\prime}}=\Sigma u_{s^{\prime}}^{h^{\prime}} \Gamma^{s^{\prime}},
\end{array} \quad(h, m=1, \ldots, n),
$$

car la matrice $\left\|u_{s^{\prime}}^{h^{\prime}}\right\|$ est la contragrédiente de la matrice $\left\|u_{s}^{h}\right\|$.

On a par exemple (cf. (5.31))

$$
\begin{aligned}
& \tilde{A}_{m p}^{h}=\Sigma u_{s}^{h} u_{v^{\prime}}^{m^{\prime}} u_{t^{\prime}}^{p^{\prime}} A_{l t}^{s}, \quad \tilde{B}_{m p}^{h}=\Sigma u_{s}^{h} u_{l}^{m} u_{t}^{p} B_{l t}^{s}, \\
& \tilde{R}_{m p^{\prime} q}^{h}=\Sigma u_{s}^{h} u_{t}^{p} u_{r^{\prime}}^{q^{\prime}} u_{l^{\prime}}^{m^{\prime}} R_{l l^{\prime} r}^{s}, \quad(h, m, p, q=1, \ldots, n) \text {. } \\
& \tilde{R}_{m p q}^{h}=\Sigma u_{s}^{h} u_{t^{\prime}}^{p^{\prime}} u_{r^{\prime}}^{q^{\prime}} u_{l^{\prime}}^{m^{\prime}} R_{l t r}^{s} \text {. }
\end{aligned}
$$

On obtient des condition nécessaires pour que la structure soit isotrope, en considérant des transtormations particulières de $U_{n}$ (resp. $\dot{U}_{n}$ ), des transformations diagonales par exemple; en raison des relations (23.2), on démontre que les composantes du tenseur de torsion doivent etre nulles et par suite $R_{l t r}^{s}=0, R_{l^{\prime} t^{\prime} r^{\prime}}^{s^{\prime}}=0$ d'après $(22.7 a)$; en considérant encore les transformations diagonales, on déduit des équations (23.2) que les composantes du tenseur de courbure doivent être nulles sauf $R_{s l^{\prime} l}^{s}, R_{l l^{\prime} s}^{s}, R_{s^{\prime} l l^{\prime}}^{s^{\prime}}, R_{l^{\prime} l^{\prime}}^{s^{\prime}}(s, l=1, \ldots, n)$.

En considérant maintenant des transformations telles que: $\tilde{\omega}^{h}=u_{s}^{h} \omega^{s}$, $\tilde{\omega}^{s}=u_{\mathrm{s}}^{h} \omega^{h}, \tilde{\omega}^{l}=\omega^{l}$ si $l \neq h, s$, on démontre qu'on doit avoir :

$$
R_{a l l l}^{s}=R_{s t^{\prime} t}^{s}=R_{l t^{\prime} t}^{l}, \quad R_{l l^{\prime} s}^{s}=R_{t t^{\prime} h}^{h}, \quad(s, l, t, h=1, \ldots, n)
$$

c' est-à-dire :

$$
\begin{aligned}
& \Omega_{l}^{s}=\lambda \omega^{l^{\prime}} \wedge \omega^{s}, \quad \Omega_{s}^{s}=\rho \omega^{8 \prime} \wedge \omega^{s}+\mu \Sigma \omega^{l^{\prime}} \wedge \omega^{l}, \\
& \Omega_{l^{\prime}}^{s^{\prime}}=\lambda \omega^{l} \wedge \omega^{s^{\prime}}, \quad \Omega_{s^{\prime}}^{s^{\prime}}=\rho \omega^{s} \wedge \omega^{s^{\prime}}+\mu \Sigma \omega^{l} \wedge \omega^{l^{\prime}},
\end{aligned} \quad(s, l=1, \ldots, n)
$$

$\lambda, \mu, \rho$ étant réels quelle que soit la nature de la structure. En utilisant les identités de BIaNchi, on démontre que: $\lambda=\mu=\rho=$ constante. 
Les composantes des tenseurs de courbure et de torsion étant des constantes, ces conditions nécessaires sont suffisantes pour l'isotropie et entraînent aussi l' homogénéité locale.

Si $\lambda=0$, la structure est intégrable.

Si $\lambda \neq 0$, nous allons interpréter successivement les formules obtenues pour les structures hermitiennes et parahermitiennes.

Dans le premier cas, on obtient:

$$
\begin{aligned}
& d \omega^{s}=\Sigma \omega^{l} \wedge \omega_{l}^{s}, \\
& d \omega_{l}^{s}=\Sigma \omega_{l}^{t} \wedge \omega_{l}^{s}+\lambda \bar{\omega}^{l} \wedge \omega^{s}, \quad(\text { si } s \neq l), \quad(s, l=1, \ldots, n) . \\
& d \omega_{s}^{s}=\Sigma \omega_{l}^{t} \wedge \omega_{t}^{s}+\lambda\left[\bar{\omega}^{s} \wedge \omega^{s}+\Sigma \bar{\omega}^{l} \wedge \omega^{l}\right], \quad \quad \\
& \omega_{l}^{s}+\bar{\omega}_{s}^{l}=0 .
\end{aligned}
$$

Ces équations sont vérifiées si la variété $V_{2 n}$ est l'espace hermitien elliptique (si $\lambda<0$ ) ou l'espace hermitien hyperbolique (si $\lambda>0$ ).

Soit en effet dans l'espace vectoriel complexe $C^{n+1}$ une base $e_{0}, e_{1}, \ldots, e_{n}$; on peut définir le produit scalaire $\left(e, \overline{e^{\prime}}\right)$ de deux vecteurs $e=z_{0} e_{0}+\ldots+z_{n} e_{n}$ et $e^{\prime}=z_{0}{ }^{\prime} e_{0}^{\prime}+\ldots+z_{n}^{\prime} e^{\prime}{ }_{n}$ par :

$$
\left(e, \overline{e^{\prime}}\right)=\left(z, \bar{z}^{\prime}\right)=z_{0} \bar{z}_{0}^{\prime}+\Sigma z_{s} \bar{z}_{s}^{\prime} ;
$$

(on suppose que les indices $s, l \ldots$ varient de 1 à $n$ ).

Les $z_{0}, z_{s}$ constituent un système de coordonnées homogènes de l'espace projectif complexe $P_{n}(C)$ et l'on peut normaliser ces coordonnées par la condition: $(z, \bar{z})=1$. Considérons dans $C^{n+1}$ une base variable $\varepsilon_{0}, \varepsilon_{1}, \ldots, \varepsilon_{n}$ telle que:

$$
\left(\varepsilon_{0}, \bar{\varepsilon}_{0}\right)=1, \quad\left(\varepsilon_{s}, \bar{\varepsilon}_{s}\right)=1, \quad\left(\varepsilon_{0}, \bar{\varepsilon}_{s}\right)=0, \quad\left(\varepsilon_{s}, \bar{\varepsilon}_{l}\right)=0 \text { si } s \neq l .
$$

On a :

$$
\begin{aligned}
& d \varepsilon_{0}=\varphi_{0}^{0} \varepsilon_{0}+\Sigma \varphi_{0}^{8} \varepsilon_{s}, \\
& d \varepsilon_{s}=\varphi_{s}^{0} \varepsilon_{0}+\Sigma \varphi_{s}^{l} \varepsilon_{l}, \\
& d \varphi_{0}^{0}=\Sigma \varphi_{0}^{l} \wedge \varphi_{l}^{0}, \\
& d \varphi_{0}^{l}=\Sigma \varphi_{0}^{t} \wedge \varphi_{t}^{l}+\varphi_{0}^{l} \wedge\left(\varphi_{l}^{l}-\varphi_{0}^{0}\right) . \\
& d \varphi_{l}^{s}=\Sigma \varphi_{s}^{t} \wedge \varphi_{t}^{l}+\varphi_{s}^{0} \wedge \varphi_{0}^{l},
\end{aligned} \quad(l, s=1, \ldots, n)
$$

avec:

$$
\varphi_{0}^{0}+\bar{\varphi}_{0}^{0}=0, \quad \varphi_{0}^{s}+\bar{\varphi}_{s}^{0}=0, \quad \varphi_{s}^{l}+\bar{\varphi}_{l}^{s}=0 .
$$

On peut définir dans $P_{n}(C)$ la métrique:

$$
d s^{2}=\left(d \varepsilon_{0}, d \bar{\varepsilon}_{0}\right)-\left(\varepsilon_{0}, \overline{d \varepsilon_{0}}\right)\left(\bar{\varepsilon}_{0}, d \varepsilon_{0}\right)
$$


P. LibermanN: Sur le problème d'équivalence, etc.

Si l'on pose: $\varphi_{0}{ }^{8}=\theta^{*}, \varphi_{s}^{l}=\omega_{s}^{l}$ (si $\left.s \neq l\right), \varphi_{s}^{*}-\varphi_{0}^{0}=\omega_{s}^{*}$, cette métrique pent $\mathrm{s}^{\prime}$ ecrire : $d s^{2}=\Sigma \omega^{2} \bar{\omega}^{2}$ et l'on obtient :

$d \omega^{*}=\Sigma \omega^{l} \wedge \omega_{i}^{*}$

$d \omega_{s}^{l}=\Sigma \omega_{s}^{t} \wedge \omega_{t}^{l}+\varphi_{s}^{0} \wedge \varphi_{0}^{l}=\Sigma \omega_{s}^{t} \wedge \omega_{t}^{l}-\bar{\omega}^{l} \wedge \omega^{l}, \quad(s, l=1, \ldots, n)$

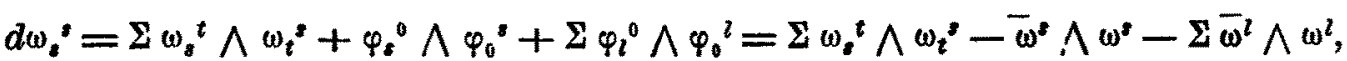

c'est-à-dire les équations $(13.3 a)$ où $\lambda=-1$.

Ce raisonnement a été indiqué par CHERN [18]. L' espace hermitien hyperbolique est obtenu de manière analogue en définissant dans $C^{n+1}$ le produit scalaire $\left(e, \bar{e}^{\prime}\right)=z_{0} z_{0}^{\prime}-\Sigma z_{s} z_{s}^{\prime}$ et considérant dans $P_{n}(C)$ la métrique:

$$
d s^{2}=\left(\varepsilon_{0}, d \bar{\varepsilon}_{0}\right)\left(\bar{\varepsilon}_{0}, d \varepsilon_{0}\right)-\left(d \varepsilon_{0}, d \bar{\varepsilon}_{0}\right)
$$

où $\varepsilon_{0}, \varepsilon_{1}, \ldots, \varepsilon_{n}$ est une base telle que:

$$
\left(\varepsilon_{0}, \bar{\varepsilon}_{\mathrm{g}}\right)=1, \quad\left(\varepsilon_{s}, \bar{\varepsilon}_{s}\right)=-1, \quad\left(\varepsilon_{0}, \varepsilon_{s}\right)=0, \quad\left(\varepsilon_{s}, \bar{\varepsilon}_{l}\right)=0 \text { si } s \neq l
$$

d'où :

$$
\varphi_{0}^{0}+\bar{\varphi}_{0}^{0}, \quad \varphi_{i}^{2}+\bar{\varphi}_{i}^{2}=0, \quad \varphi_{0}^{2}=\bar{\varphi}_{0}^{0} . \quad(s, l=1, \ldots, n) .
$$

On déduit des résultats précédents le théorème suivant:

TH'оRымe 23.1a. - Une structure presque hermitienne isotrope en chaque point est localement homogène. Cette structure est intégrable ou bien localement équivalente à la structure d'espace hermitien elliptique ou hyperbolique.

Pour les structures presque parahermitiennes, on obtient le theorème:

THÉORÈm 23.1b. - Une structure presque parahermitienne isotrope en chaque point est localement homogène. Cette structure est intégrable ou bien localement équivalente à une structure parahermitienne sur la variété $\boldsymbol{P}_{n}(R) \times P_{n}(R)$, appelée structure d'espace parahermitien.

Les relations (23.3) peuvent en effet s'écrire pour les structures parahermitiennes (les formes $\omega^{*}$, $\omega^{s \prime}$ étant réelles).

$$
\begin{aligned}
& d \omega^{*}=\Sigma \omega^{l} \wedge \omega_{l}^{*}, \quad d \omega^{8 \prime}=\Sigma \omega^{l^{\prime}} \wedge \omega_{l^{\prime}}^{z^{\prime}}, \\
& d \omega_{l}^{s}=\Sigma \omega_{l}^{t} \wedge \omega_{l}^{l}+\lambda \omega^{l^{\prime \prime}} \wedge \omega^{s}, \quad d \omega_{l^{\prime}}^{s^{\prime}}=\Sigma \omega_{t^{\prime}}^{l^{\prime}} \wedge \omega_{t^{\prime}}^{z^{\prime}}+\lambda \omega^{l} \wedge \omega^{s}, \\
& d \omega_{s}^{2}=\Sigma \omega_{s}^{t} \wedge \omega_{t}^{z}+\lambda \omega^{s^{\prime}} \wedge \omega^{s}+\lambda \Sigma \omega^{l^{\prime}} \wedge \omega^{l}, \quad d \omega_{s}^{s^{\prime}}=\Sigma \omega_{s^{\prime}}^{t^{\prime}} \wedge \omega_{t^{\prime}}^{s^{\prime}}+\lambda \omega^{z} \wedge \omega^{\prime \prime}+\lambda \Sigma \omega^{2} \wedge \omega^{\prime \prime}, \\
& \omega_{l}{ }^{8}+\omega_{s^{\prime}}{ }^{\prime \prime}=0 \quad(s, l=1, \ldots, n),
\end{aligned}
$$


où l'on peut supposer $\lambda<0$ : en chaque point l'automorphisme contragrédient de $\mathfrak{F}_{x}$ (qui transforme $\Omega_{x}$ en $-\Omega_{x}$ ) conserve les formes $\omega^{*}$, transforme les $\omega^{s^{\prime}}$ en $-\omega^{s \prime}$ et par conséquent transforme $\lambda$ en $-\lambda$; on obtient la même espèce de structure quel que soit le signe de $\lambda$ contrairement au cas hermitien.

Soit dans l'espace rectoriel $R^{2 n+2}$ une structure de module unitaire paracomplexe (\$11) définie par deux sous-espaces supplémentaires $X_{n+1}$ et $X_{n+1}^{\prime}$; soit $e_{0}, e_{1}, \ldots, e_{n}$ une base dans $X_{n+1}$ et $e_{0}^{\prime}, e_{1}^{\prime}, \ldots, e_{n}^{\prime}$ une base dans $X_{n+1}^{\prime}$. A deux vecteurs quelconques $e=u^{\prime \prime} e_{0}+\Sigma u^{8} e_{g}$ et $e^{\prime}=u^{\prime 0} e_{0}^{\prime}+\Sigma u^{s} e_{s}^{\prime}$, on peut associer le produit scalaire $\left(e, e^{\prime}\right)=\Sigma u^{s} u^{s}+u^{0} u^{\prime 0}$.

Considérons les bases variables $\varepsilon_{0}, \varepsilon_{1}, \ldots, \varepsilon_{n}$ dans $X_{n+1}$ et $\varepsilon_{0}^{\prime}, \varepsilon_{1}^{\prime}, \ldots, \varepsilon_{n}^{\prime}$ dans $X_{n+1}^{\prime}$ telles que:

$$
\left(\varepsilon_{0}, \varepsilon_{0}^{\prime}\right)=1, \quad\left(\varepsilon_{s}, \varepsilon_{s}^{\prime}\right)=1, \quad\left(\varepsilon_{0}, \varepsilon_{s}^{\prime}\right)=0, \quad\left(\varepsilon_{s}, \varepsilon_{l}^{\prime}\right)=0 \text { si } s \neq l .
$$

On a :

$$
\begin{array}{ll}
d \varepsilon_{0}=\varphi_{0}^{0} \varepsilon_{0}+\Sigma \varphi_{s}^{0} \varepsilon_{s}, & d \varepsilon_{0}^{\prime}=\varphi_{0}^{\prime 0} \varepsilon_{0}^{\prime}+\Sigma \varphi_{0^{\prime}}^{s^{\prime}} \varepsilon_{s}^{\prime}, \\
d \varepsilon_{s}=\varphi_{s}^{0} \varepsilon_{0}+\Sigma \varphi_{s}^{l} \varepsilon_{l}, & d \varepsilon_{s}^{\prime}=\varphi_{s^{\prime}}^{0 \prime} \varepsilon_{0}^{\prime}+\Sigma \varphi_{s^{\prime}}^{l^{\prime}} \varepsilon_{l}^{\prime}, \\
d \varphi_{0}^{0}=\Sigma \varphi_{0}^{l} \wedge \varphi_{l}^{0}, & d \varphi_{0}^{\prime 0}=\Sigma \varphi_{0}^{l^{\prime}} \wedge \varphi_{l^{\prime}}^{0 \prime}, \\
\left.d \varphi_{0}^{s}=\Sigma \varphi_{0}^{t} \wedge \varphi_{t}^{s}+\varphi_{0}^{s} \wedge \varphi_{s}^{s}-\varphi_{0}^{0}\right), & d \varphi_{0}^{s^{\prime}}=\Sigma \varphi_{0^{\prime}}^{t^{\prime}} \wedge \varphi_{t^{\prime}}^{s^{\prime}}+\varphi_{0^{\prime}}^{s^{\prime}} \wedge\left(\varphi_{s^{\prime}}^{s \prime}-\varphi_{0}^{\prime 0}\right) \\
d \varphi_{s}^{l}=\Sigma \varphi_{s}^{t} \wedge \varphi_{t}^{l}+\varphi_{s}^{0} \wedge \varphi_{0}^{l}, & d \varphi_{l^{\prime}}^{s^{\prime}}=\Sigma \varphi_{s^{\prime}}^{t^{\prime}} \wedge \varphi_{t^{\prime}}^{l^{\prime}}+\varphi_{s^{\prime}}^{0 \prime} \wedge \varphi_{0^{\prime}}^{l^{\prime}},
\end{array}
$$

avec

$$
\varphi_{0}^{0}+\varphi_{0}^{\prime 0}=0, \quad \varphi_{0^{\prime}}{ }^{\prime \prime}+\varphi_{s}^{0}=0, \quad \varphi_{g^{\prime}} l^{\prime \prime}+\varphi_{l}^{s}=0 . \quad(s, l=1, \ldots, n) .
$$

On peut définir dans l'espace projectif paracomplexe $P_{n}(A)$ (cf. $\S 11$ ) c'est-à-dire dans $P_{n}(R) \times P_{n}(R)$ la métrique suivante:

$$
d s^{2}=\left(d \varepsilon_{0}, d \varepsilon_{0}\right)-\left(\varepsilon_{0}, d \varepsilon_{0}^{\prime}\right)\left(\varepsilon_{0}^{\prime}, d \varepsilon_{0}\right) .
$$

Si l'on pose: $\varphi_{0}^{8}=\omega^{s}, \varphi_{s}{ }^{l}=\omega_{s}{ }^{l}$ (si $\left.s \neq l\right) \varphi_{s}^{s}-\varphi_{0}^{0}=\omega_{s}^{8}, \quad \varphi_{0^{\prime}}{ }^{8}=\omega^{8 \prime}$, $\varphi_{s^{\prime}}{ }^{\prime \prime}=\omega_{s^{\prime}}{ }^{\prime \prime}, \varphi_{s^{\prime}}{ }^{\prime \prime}-\varphi_{0}^{\prime 0}=\omega_{g^{\prime}} s^{\prime}$, la métrique peut $s^{\prime}$ écrire: $d s^{2}=\Sigma \omega^{l} \omega^{l^{\prime \prime}}$ et l' on obtient les equations (23.3b) où $\lambda=-1$. Appelons espace parahermitien l'espace projectif paracomplexe muni de cette métrique.

Si l'on se borne à une isotropie restreinte telle que tont corepère d'orisine $x$ puisse être transformée en un corepère se déduisant du premier par une transformation du groupe unitaire (resp. para-unitaire) unimodulaire (le groupe para-unitaire unimodulaire etant le sous-groupe de $\stackrel{\circ}{n}_{n}$ isomorphe an groupe linéaire homogène réel unimodulaire), le même raisonnement que pour les structures isotropes conduit aux structures définies par le théorème 23.1 et, en outre, pour $n=3$, à une structure déterminée localement par 
des formes $\omega^{s}, \omega^{s \prime}$ vérifiant les équations:

$$
\begin{aligned}
& d \omega^{1}=\omega^{1} \wedge \omega_{1}^{1}+\omega^{2} \wedge \omega_{2}^{1}+\omega^{3} \wedge \omega_{3}^{1}-2 \rho \omega^{5} \wedge \omega^{6}, \\
& d \omega^{2}=\omega^{1} \wedge \omega_{1}^{2}+\omega^{2} \wedge \omega_{2}^{2}+\omega^{3} \wedge \omega_{3}^{2}-2 \rho \omega^{6} \wedge \omega^{4} \\
& d \omega^{3}=\omega^{1} \wedge \omega_{1}^{3}+\omega^{2} \wedge \omega_{2}^{3}+\omega^{3} \wedge \omega_{3}^{3}-2 \rho \omega^{4} \wedge \omega^{5} \\
& d \omega^{4}=\omega^{4} \wedge \omega_{4}^{4}+\omega^{5} \wedge \omega_{5}^{4}+\omega^{6} \wedge \omega_{6}^{4}-2 \rho^{\prime} \omega^{2} \wedge \omega^{3} \\
& d \omega^{5}=\omega^{4} \wedge \omega_{4}^{5}+\omega^{5} \wedge \omega_{5}^{5}+\omega^{6} \wedge \omega_{6}^{5}-2 \rho^{\prime} \omega^{3} \wedge \omega^{1} \text {, } \\
& d \omega^{6}=\omega^{4} \wedge \omega_{4}^{6}+\omega^{5} \wedge \omega_{5}^{6}+\omega^{6} \wedge \omega_{6}^{6}-2 \rho^{\prime} \omega^{1} \wedge \omega^{2} \text {, } \\
& \Sigma \omega_{s}{ }^{s}=0, \quad \omega_{s}{ }^{l}+\omega_{l^{\prime}}{ }^{g}=0 \text {, } \\
& d \omega_{s}{ }^{l}=\Sigma \omega_{s}^{t} \wedge \omega_{t}^{l}-3 \rho \rho^{\prime} \omega^{s t} \wedge \omega^{l}, \quad(l, s=1,2,3) \\
& d \omega_{s}^{s}=\Sigma \omega_{s}{ }^{t} \wedge \omega_{t}{ }^{g}-3 \rho \rho^{\prime} \omega^{s t} \wedge \omega^{g}+\rho \rho^{\prime} \Sigma \omega^{l t} \wedge \omega^{l},
\end{aligned}
$$

On est d'abord conduit à des structures pour lesquelles les formes $\omega^{s}$, $\omega^{s \prime}$ vérifient les equations $(23.7)$ et la relation $\rho \rho^{\prime}=$ constante, mais on peut choisir en chaque point une classe de corepères unitaires (resp. para-unitaires) modulo le groupe unitaire (resp. para-unitaire) tels que $\rho=\rho^{\prime}=$ constante.

Si la structure est presque hermitienne, les formes $\omega^{8}$, $\omega^{8 /}$ sont complexes avec $\omega^{s^{\prime}}=\bar{\omega}^{s}$. Les equations $(23.7)$ et $(23.8)$ sont les équations de structure du groupe simple compact $G_{2}$ à 14 paramètres qui opère transiti. vement sur la sphère $S_{6}$. Si la structure est presque parahermitienne, les formes $\omega^{s}, \omega^{s}$ sont réelles et l'on obtient les équations de structure du groupe simple $G_{2}{ }^{\prime}$ qui est la deuxième forme réelle du groupe complexe dont $G_{2}$ est la forme, réelle compacte [8], [11]. Ce groupe $G_{2}^{\prime}$ opère transitivement sur la quadrique $Q_{6}$ d'équation: $Z^{2}+X^{1} Y^{1}+X^{2} Y^{2}+X^{3} Y^{3}=1$.

On en déduit:

THÉonéme 23.2. - Une structure presque hermitienne (resp. presque parahermitienne) isotrope d'une manière restreinte en chaque point est ou bien isotrope ou bien localement équivalente à une structure presque hermitienne $\mathrm{g}$ (resp. presque parahermitienne $\mathfrak{g}^{\prime}$ ) sur la sphère $S_{6}$ (resp. la quadrique réelle $Q_{8}$ ) admettant le groupe $G_{2}$ (resp. $G_{2}{ }^{\prime}$ ) comme groupe d'automorphismes.

Le deuxième torsion n'étant pas nulle, la structure hermitienne $s$ sur $S_{6}$ définie par les équations (23.7) et (23.8) ne dérive pas d'une structure complexe. Comme $G_{2}$ ne laisse invariante sur $S_{6}$ qu'une seule structure presque complexe, la structure $s$ est isomorphe à la structure définie à l'aide des octaves de Cayley [32], e'est-à-dire d'une algèbre sur le corps des réels de base $\left(1, e_{1}, \ldots, e_{7}\right)$ telle que :

$$
\begin{aligned}
& \left(e_{s}\right)^{2}=-1, e_{s} e_{l}=-e_{l} e_{s} \text { si } l \neq s, e_{s}\left(e_{s} e_{l}\right)=\left(e_{s}\right)^{2} e_{l} \\
& e_{s}=e_{s+1} e_{s+3}=e_{s+3} e_{s+8}=e_{s+4} e_{s+5} \text { où } e_{s+\gamma}=e_{s}
\end{aligned} \quad(s=1, \ldots, 7) .
$$


D'autre part comme tout espace localement homogène de LIE compact et simplement connexe est équivalent à un espace homogène de LIE [23] et qu'en dehors du groupe orthogonal, $G_{2}$ est le seul groupe de LIE compact opérant transitivement sur $S_{6}[4]$, toute structure localement homogène sur $S_{6}$ est équivalente à la structure $\mathrm{g}$.

La structure $\mathfrak{s}^{\prime}$ sur $Q_{6}$ pent ètre déterminée par la donnée de deux champs de génératrices supplémentaires de dimension 3 ; la deuxième torsion n'étant pas nulle, ces champs ne sont pas complètement intégrables, c'est-àdirè la structure ne dérive pas d'une structure paracomplexe; on peut d'ailleurs démontrer qu'il n'existe, en dehors des génératrices elles-mêmes, aucune sous-variété à 3 dimensions de $Q_{6}$ tangente à un champ de génératrices. La structure $\mathfrak{g}^{\prime}$ peut également être définie à l'aide d'octaves de Cayley de deuxième espèce, en désignant par octaves de CAYLEY de deuxième espèce les éléments d'une algèbre sur le corps réels de base $\left(1, e_{1}, \ldots, e_{7}\right)$ telle que:

$$
\begin{aligned}
& e_{8} e_{l}=-e_{l} e_{8} \text { si } s \neq l, \quad e_{8}\left(e_{s} e_{l}\right)=\left(e_{8}\right)^{2} e_{l} \quad(s, l=1, \ldots, 7) \\
& \left(e_{1}\right)^{2}=\left(e_{4}\right)^{2}=\left(e_{6}\right)^{2}=\left(e_{7}\right)^{2}=1, \quad\left(e_{2}\right)^{2}=\left(e_{3}\right)^{2}=\left(e_{5}\right)^{2}=-1, \\
& e_{1} e_{4}=e_{2}, \quad e_{1} e_{3}=e_{7}, \quad e_{1} e_{5}=e_{6}, \quad e_{4} e_{3}=-e_{6}, \quad e_{4} e_{5}=e_{7}, \\
& e_{6} e_{2}=-e_{7}, \quad e_{2} e_{3}=e_{5} .
\end{aligned}
$$

Soit l'octave de deuxième espèce $x=x^{0}+x^{4} e_{1}+x^{2} e_{2}+x^{3} e_{3}+x^{4} e_{4}+$ $+x^{5} e_{5}+x^{6} e_{6}+x^{7} e_{7} ; x^{0}$ est la partie réelle (notée $R e x$ ) et $x-x^{0}$ sa partie imaginaire pure; le conjugué de $x$ est egal $x: \vec{x}=x^{0}-x^{4} e_{1}-x^{2} e_{2}-x^{3} e_{3}-$ $-x^{4} e_{4}-x^{5} e_{5}-x^{6} e_{6}-x^{7} e_{7} ;$ la norme de $x$ est :

$$
\begin{aligned}
& N(x)=x \bar{x}=\left(x^{0}\right)^{2}+\left(x^{1}\right)^{2}+\left(x^{4}\right)^{2}+\left(x^{6}\right)^{2}+\left(x^{7}\right)^{2}-\left(x^{2}\right)^{2}-\left(x^{3}\right)^{2}- \\
& -\left(x^{5}\right)^{2}=\left(x^{0}\right)^{2}+\left(x^{1}\right)^{2}+\left(x^{4}-x^{2}\right)\left(x^{4}+x^{2}\right)+\left(x^{6}-x^{5}\right)\left(x^{6}+x^{5}\right)+ \\
& +\left(x^{7}-x^{3}\right)\left(x^{7}+x^{3}\right) .
\end{aligned}
$$

On peut définir le produit scalaire de deux octaves $x$ et $y$ par:

$$
\begin{aligned}
& (x, y)=\operatorname{Re}(x \bar{y})=\operatorname{Re}(\bar{x} y)=x^{0} y^{0}+x^{1} y^{1}+x^{4} y^{4}+x^{6} y^{6}+x^{7} y^{7}-x^{2} y^{2}- \\
& -x^{3} y^{3}-x^{5} y^{5}
\end{aligned}
$$

Tout point de l'espace numérique $R^{T}$ peut être représenté par un octave de deuxième espèce imaginaire pur $x=x^{1} e_{1}+x^{2} e_{2}+x^{3} e_{3}+x^{4} e_{4}+x^{5} e_{5}+x^{6} e_{6}+x^{7} e_{7}$. Tout point de la quadrique $Q_{\varepsilon}$ peut etre représenté par un octare imaginaire pur, de norme égale à 1 . Si $x$ et $y$ représentant deux points de $Q_{6}$ on a : $x y=-y x$ et dans l'hyperplan orthogonal à $x$ (qui se déduit par translation du plan tangent $T_{x}$ à $Q_{6}$ en $x$ ), la multiplication à gauche par $x$ est une (3.3)-involution $\mathfrak{F}_{x}$.

On peut également chercher à déterminer des structures presque hermitiennes d'espèce $k$ qui soient isotropes ou localement homogènes. Un raison. nement analogue à celui utilisé pour les structures presque hermitiennes ou 
presque parahermitiennes conduit au structures intégrables ou à des structures définies localement par les formes complexes $\omega^{s}$, $\omega^{A}$ telles que la forme d'Hermine associée à la structure puisse s'écrire:

$$
\Phi^{\langle k\rangle}=\Sigma \omega^{s} \bar{\omega}^{s}-\Sigma \omega^{A} \bar{\omega}^{A},
$$

et vérifiant les équations :

$$
\begin{aligned}
& d \omega^{s}=\Sigma\left(\omega^{l} \wedge \omega_{l}^{s}+\omega^{B} \wedge \omega_{B}^{s}\right) \\
& d \omega^{A}=\Sigma\left(\omega^{l} \wedge \omega_{l}^{A}+\omega^{B} \wedge \omega_{B}^{A}\right), \\
& d \omega_{l}^{s}=\Sigma\left(\omega_{l}^{t} \wedge \omega_{l}^{s}+\omega_{l}^{c} \wedge \omega_{c}^{b}\right)+\lambda \bar{\omega}^{l} \wedge \omega^{s} \text {, } \\
& d \omega_{s}^{s}=\Sigma\left(\omega_{s}^{t} \wedge \omega_{t}^{s}+\omega_{s}^{C} \wedge \omega_{C}^{s}\right)+\lambda \bar{\omega}^{s} \wedge \omega^{s}+\lambda \Sigma\left(\bar{\omega}^{l} \wedge \omega^{l}-\bar{\omega}^{B} \wedge \omega^{B}\right) \\
& d \omega_{B}^{A}=\Sigma\left(\omega_{B}^{t} \wedge \omega_{t}^{A}+\omega_{B}^{C} \wedge \omega_{C}^{A}\right)-\lambda \bar{\omega}^{B} \wedge \omega^{A} \text {, } \\
& d \omega_{A}^{A}=\Sigma\left(\omega_{A}^{t} \wedge \omega_{t}^{A}+\omega_{A}^{C} \wedge \omega_{C}^{A}\right)-\lambda \bar{\omega}^{A} \wedge \omega^{A}+\lambda \Sigma\left(\bar{\omega}^{l} \wedge \omega^{l}-\bar{\omega}^{B} \wedge \omega^{B}\right) \\
& d \omega_{A}^{s}=\Sigma\left(\omega_{A}^{t} \wedge \omega_{t}^{s}+\omega_{A}^{C} \wedge \omega_{C}^{s}\right)-\lambda \bar{\omega}^{A} \wedge \omega^{s} \text {, } \\
& d \omega_{s}^{A}=\Sigma\left(\omega_{s}^{t} \wedge \omega_{t}^{A}+\omega_{s}^{C} \wedge \omega_{C}^{A}\right)+\lambda \bar{\omega}^{s} \wedge \omega^{A} \text {, } \\
& \omega_{s}^{l}+\bar{\omega}_{l}^{s}=0, \quad \bar{\omega}_{B}^{A}+\bar{\omega}_{A}^{B}=0, \quad \omega_{s}^{A}+\bar{\omega}_{A}^{s}=0,
\end{aligned}
$$

(les indices minuscules $s, l, t$ variant de 1 à $k$, les indices majuscules $A, B, C$ de $k+1$ à $n$ ).

On peut supposer la constante $\lambda$ négative, en remplaçant au besoin la

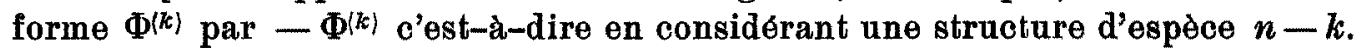

Désignons par espace hermitien $d$ 'espèce $k$ l'espace projectif complexe $\boldsymbol{P}_{n}(C)$ dans lequel on définit les coordonnées normales par la condition:

$$
(z, \bar{z})=z_{0} \bar{z}^{0}+z_{1} \bar{z}_{1}+\ldots+z_{k} \bar{z}_{k}-z_{k+1} \bar{z}_{k+1}-\ldots-z_{n} \bar{z}_{n}=1 \text {; }
$$

on définit ainsi dans $C^{n+1}$ le produit scalaire $\left(e, e^{\prime}\right)=z_{0} z_{0}{ }^{\prime}+\Sigma z_{s} \bar{z}_{s}^{\prime}-\Sigma z_{A} \bar{z}_{A}^{\prime}$.

Si l'on choisit dans $C^{n+1}$ une base variable $\varepsilon_{0}, \varepsilon_{1}, \ldots, \varepsilon_{n}$ telle que:

$$
\begin{gathered}
\left(\varepsilon_{0}, \bar{\varepsilon}_{0}\right)=\left(\varepsilon_{s}, \bar{\varepsilon}_{s}\right)=1, \quad\left(\varepsilon_{A}, \bar{\varepsilon}_{A}\right)=-1, \quad\left(\varepsilon_{0}, \bar{\varepsilon}_{s}\right)=\left(\varepsilon_{0}, \bar{\varepsilon}_{A}\right)=0,\left(\varepsilon_{s}, \bar{\varepsilon}_{A}\right)=0, \\
\left(\varepsilon_{s}, \bar{\varepsilon}_{l}\right)=0 \text { si } s \neq l, \quad\left(\varepsilon_{A}, \bar{\varepsilon}_{B}\right)=0 \text { si } A \neq B,
\end{gathered}
$$

et si l'on définit dans $P_{n}(C)$ la métrique: $\left(d \varepsilon_{s}, d \bar{\varepsilon}_{0}\right)-\left(\varepsilon_{0}, d \bar{\varepsilon}_{0}\right)\left(\bar{\varepsilon}_{0}, d \varepsilon_{0}\right)$, le même raisonnement que pour l'espace hermitien elliptiqne conduit aux équa. tions $(23.3 c)$ arec $\lambda=-1$; d'où :

THÉonime 23.1c. - Une structure presque hermitienne d'espèce k est localement homogène; cette structure est intégrable ou bien localement équivalente à la structure d'espace hermitien d' espèce $k$.

L'espace hermitien d'espèce $k$ est un espace riemannien symétrique à métrique indéfinie.

Les structures presque hermitiennes d'espèce $k$ isotropes d'une manière restreinte sont isotropes; d'ailleurs les groupes $G_{2}$ et $G_{2}^{\prime}$ sont les deux seules formes réelles du groupe simple complexe exceptionnel à 14 paramètres [11]. 
24. Courbure scalaire [38] et [41].

Soit sur une variété $V_{2 n}$ une structure presque hermitienne (déterminée par une métrique riemannienne $F$ et un champ d'automorphismes $\mathfrak{J}_{x}$ ). Dans l'espace tangent $T_{x}$ à $V_{2 n}$, muni de sa structure d'espace vectoriel complexe considérons deux vecteurs $V$ et $W$ de composantes $\xi^{1}, \ldots, \xi^{n} ; \eta^{1}, \ldots, \eta^{n}$ par rapport à un repère unitaire (nous adoptons encore la convention: $\xi^{\prime}=\overline{\xi^{s}}$, $\left.\eta^{s^{\prime}}=\bar{\eta}^{s}\right)$. L'ensemble des vecteurs $m V+p W$ tels que le rapport $p / m$ soit réel engendre un élément plan $P$ à deux dimensions réelles; si en particulier $P$ est invariant par l'automorphisme $\mathfrak{g}_{x}, P$ est une droite complexe de $T_{x}{ }^{c}$; $P$ peut alors etre engendré par les vecteurs $V$ et $\mathfrak{I}_{x} V$.

De mème soit sur une variété $V_{2 n}$ une structure presque parahermitienne (déterminée par une forme différentielle extérieure $\Omega$ et un champ d'involutions $\mathfrak{F}_{x}$ ). Dans l'espace tangent $T_{x}$, soit un élément plan $P$ engendré par deux vecteurs $V$ et $W$ de composantes : $\xi^{1}, \ldots, \xi^{n}, \xi^{\prime 1}, \ldots, \xi^{n} ; \eta^{1}, \ldots, \eta^{n}, \eta^{\prime 1}, \ldots, \eta^{\prime n}$ par rapport à un repère para-unitaire tel que les vecteurs $e_{s}$ engendrent le sous-espace positif de $\mathscr{F}_{x}$, les vecteurs $e_{s^{\prime}}$ son sous-espace négatif. Si $P$ est invariant par l'automorphisme $\mathfrak{F}_{x}$ (c'est-à-dire si $P$ est une droite paracomplexe tangente à $V_{2 n}$ ), il peut être engendré par les vecteurs $V$ et $\mathfrak{G}_{x} V$.

Dans l'élément plan $P$ on pent définir la courbure scalaire de la structure presque hermitienne (resp. presque parahermitienne) par (cf. [2]):

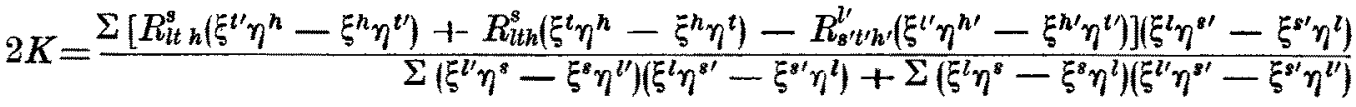

$$
\begin{aligned}
& +\frac{\left.\Sigma\left[R_{l^{\prime} h^{\prime}}^{s^{\prime}}\left(\xi^{t} \eta^{h^{\prime}}-\xi^{h^{\prime}} \eta^{t}\right)+R_{l^{\prime} t^{\prime} h^{\prime}}^{s^{\prime}} \xi^{t^{\prime}} \eta^{h^{\prime}}-\xi^{h^{\prime}} \eta^{t^{\prime}}\right)-R_{s t h}^{l}\left(\xi^{t} \eta^{n}-\xi^{h} \eta^{t}\right)\right]\left(\xi^{l^{\prime}} \eta^{s}-\xi^{s} \eta^{\prime \prime}\right)}{\left.\left.\Sigma\left(\xi^{\prime \prime} \eta^{s}-\xi^{s} \eta^{l^{\prime}}\right)\right) \xi^{\xi^{l} \eta^{8 \prime}}-\xi^{s^{\prime}} \eta^{l}\right)+\Sigma\left(\xi^{l} \eta^{s}-\xi^{s} \eta^{l}\right)\left(\xi^{l^{\prime}} \eta^{s \prime}-\xi^{s^{\prime}} \eta^{l^{\prime}}\right)}
\end{aligned}
$$

soit :

$$
K=\frac{\Sigma\left[R_{l t^{\prime} h}^{s}\left(\xi^{t^{\prime}} \eta^{h}-\xi^{h} \eta^{t^{\prime}}\right)+R_{l t h}^{s}\left(\xi^{t} \eta^{h}-\xi^{h} \eta^{t}\right)-R_{s^{\prime} t^{\prime} h}^{l^{\prime}}\left(\xi^{t^{\prime}} \eta^{h^{\prime}}-\xi^{h^{\prime}} \eta^{t^{\prime}}\right)\right]\left(\xi^{l} \eta^{s^{\prime}}-\xi^{s^{\prime}} \eta^{l}\right)}{\Sigma\left(\xi^{l^{\prime}} \eta_{1}^{s}-\xi^{s} \eta^{l^{\prime}}\right)\left(\xi^{l} \eta^{s^{\prime}}-\xi^{s^{\prime}} \eta^{l}\right)+\Sigma\left(\xi^{l} \eta^{s}-\xi^{s} \eta^{l}\right)\left(\xi^{l^{\prime}} \eta^{s^{\prime}}-\xi^{s^{\prime}} \eta^{l^{\prime}}\right)}
$$

car $\Omega_{l}^{*}+Q_{s^{\prime}}^{b \prime}=0$. Done $k$ est réel même pour les structures presque hermitiennes.

La courbure scalaire est définie dans tout élément plan si la structure est presque hermitienne; dans le cas d'une structure presque parahermitienne, le scalaire de courbure n' est pas défini dans un élément plan situé dans le sous-espace positif ou négatif de $\mathfrak{F}_{x}$. On vérifie que le scalaire $K$ défini par (24.1) est bien indépendant du repère unitaire (resp. para-unitaire); par contre l'automorphisme $\mathfrak{F}_{x}$ (transformation n'appartenant pas à $\dot{U}_{n}$ ) change $K$ en $-K$.

Théorìme 24.1. - Si en chaque point $x \in V_{2 n}$, le scalaire de courbure est indépendant de l'élément plan $P$ passant par ce point, ce scalaire est nul. 
Pour démontrer ce théorème on considère des plans engendrés par les vecteurs $V$ et $W$ dont les seules composantes non nulles sont: $\xi^{l}, \xi^{\prime}, \xi^{s}, \xi^{s^{\prime}}$; $\eta^{l}, \eta^{l^{\prime}}, \xi^{l}, \xi^{l^{\prime}}$; la condition imposée entraîne:

$R_{l l^{\prime} s}^{s}+R_{s s^{\prime} l}^{l}=4 K, \quad R_{l^{\prime} s}^{s}+R_{s s^{\prime} l}^{l}+R_{s l^{\prime} l}^{s}+R_{l s^{\prime} s}^{l}=-K, \quad R_{l l^{\prime} l}^{l}+R_{s s^{\prime} s}^{s}=-2 K$, d'où $K=0$.

Par suite on a :

$$
\begin{aligned}
& R_{l t^{\prime} h}^{s}+R_{h s^{\prime} l}^{t}=0, \quad R_{l t h}^{s}+R_{l l h}^{s}=0, \quad R_{l t h}^{s}+R_{h t l}^{s}=0, \\
& R_{l^{\prime} t h^{\prime}}^{s^{\prime}}+R_{h^{\prime} l^{\prime}}^{s}=0, \quad R_{l^{\prime} t^{\prime} h^{\prime}}^{s}+R_{t^{\prime} h^{\prime}}^{s}=0, \quad R_{l^{\prime} h^{\prime}}^{s^{\prime}}+R_{h^{\prime} t^{\prime} t^{\prime}}^{s^{\prime}}=0,
\end{aligned}
$$

La condition $K=0$ n'implique pas nécessairement que la tenseur de courbure soit nul. Nous ferons sur la structure des hypothèses supplémentai. res permettant de conclure que ce tenseur est nul.

Supposons d'abord la structure kählérienne (resp. parakählérienne); dans ce cas la torsion étant nulle, en raison des identités de BrancHI on a : $\Sigma \omega^{l} \wedge Q_{l}^{s}=0$, d'où $R_{l t^{\prime} h}^{s}=R_{h s^{\prime} l}^{t}, R_{l^{\prime} h^{\prime}}^{g^{\prime}}=R_{h^{\prime} l^{\prime}}^{t^{\prime}} ;$ donc si $K=0$, d'après $(24.2)$.

$$
R_{l t^{\prime} h}^{s}=0 \quad R_{l l^{\prime} h^{\prime}}^{s^{\prime}}=0 \quad(s, l, t, h=1, \ldots, n),
$$

et la structure est intégrable; d' où :

THÉoRÉme 24.2. - Les structures kählériennes (resp. parakählériennes) da courbure scalaire nulle sont intégrables.

Les théorèmes 24.1 et 24.2 on été démontrés par Bochner en supposant la variêté kăhlérienne [2].

Si la première torsion d'une structure presque hermitienne (resp. presque parahermitienne) est nulle, nous avons démontré que: $R_{l t h}^{s}+R_{t h l}^{s}+R_{h l t}^{s}=0$; comme $R_{l t h}^{s}+R_{l h t}^{s}=0$, si $K=0$, en raison des formules (14.2) on a: $R_{l t h}^{s}=0$ $(s, l, t, h=1, \ldots, n)$; ces relations sont également vérifiées lorsque la deuxième torsion seule est nulle (cf. $\S 22$ ). Par suite si l'une des torsions est nulle et si $K=0$, on a $\Omega_{s}^{l}=\Sigma R_{l t^{\prime} h \omega^{\prime \prime}} \wedge \omega^{n}$; les relations (24.2) entraînent alors: $\Sigma \Omega_{l}^{s} \wedge \omega^{l} \wedge \omega^{s \prime}=0$, d'où en raison des identités de BraNchr :

$\Sigma d \Omega^{s} \wedge \omega^{s^{\prime}}+\Sigma \Omega^{l} \wedge \omega_{l}^{a} \wedge \omega^{s^{\prime}}=0$, ou bien $\Sigma d \Gamma^{s} \wedge \omega^{s^{\prime}}+\Sigma \Gamma^{l} \wedge \omega_{l}^{s} \wedge \omega^{s^{\prime}}=0$, $\Sigma d \Omega^{s^{\prime}} \wedge \omega^{s}+\Sigma Q^{y^{\prime}} \wedge \omega_{l^{\prime}}^{s^{\prime}} \wedge \omega^{s}=0, \quad$ ou bien $\Sigma d \Gamma^{s^{\prime}} \wedge \omega^{s}+\Sigma \Gamma^{l^{\prime}} \wedge \omega_{l^{\prime}}^{s^{\prime}} \wedge \omega^{s}=0$; ces relation peuvent s'écrire :

$$
\begin{array}{ll}
\Sigma d\left(\Omega^{s} \wedge \omega^{s^{\prime}}\right)-\Sigma \Omega^{s} \wedge \Omega^{s^{\prime}}=0, & \Sigma d\left(\Gamma^{s} \wedge \omega^{s^{\prime}}\right)-\Sigma \Gamma^{s} \wedge \Gamma^{s^{\prime}}=0 \\
\Sigma d\left(\Omega^{s^{\prime}} \wedge \omega^{s}\right)-\Sigma \Omega^{s^{\prime}} \wedge \Omega^{s}=0, & \Sigma d\left(\Gamma^{s^{\prime}} \wedge \omega^{s}\right)-\Sigma \Gamma^{s^{\prime}} \wedge \Gamma^{s}=0 .
\end{array}
$$

En particulier si $d \Omega=0$, on a : $\Omega^{s}=\Omega^{s^{\prime}}=0$ et $\Sigma \Gamma^{s} \wedge \omega^{s^{\prime}}=0$, $\Sigma \Gamma^{s^{\prime}} \wedge \omega^{s}=0$; si de plus $K=0$, on obtient: $\Sigma \Gamma^{s} \wedge \Gamma^{s^{\prime}}=0$; on en déduit notamment : $\Sigma B_{l t}^{s} B_{l t}^{s^{\prime}}=0(l, t=1, \ldots, n)$.

Si la structure est presque kăhlérienne, les relations $\Sigma B_{l t}^{s} \bar{B}_{l t}^{s}=0$ entraînent $B_{l t}^{s}=0$; les deux torsions sont done nulles et d'après le théorème 24.2, le tenseur de courbure est nul. D'où : 
THonione 24.3. - Les structures presque kählériennes à courbure scalaire nulle sont intégrables.

Lorsque la structure est presque parakăhlérienne les relations $\Sigma B_{\imath t} B_{l^{\prime} t^{\prime}}^{s^{\prime}}=0$

n'entrafnent pas nécessairement que la deuxième torsion suit nulle. Supposons de plus que l'un des champs définissant la structure soit complètement integrable, $C_{1}$ par exemple; si $\Gamma^{* \prime}=0$, la première torsion étant nulle, on en déduit : $\Sigma \omega^{l^{\prime}} \wedge \Omega_{l^{\prime}}^{s^{\prime}}=0$, d' où : $R_{l^{\prime} t h}^{s^{\prime}}=R_{h^{\prime} s l}^{t^{\prime}}$; si $K=0$, le tenseur de courbure est nul; donc les structures presque parakăhlériennes à courbure scalaire nulle et telles que l'un des champs de $n$-éléments qui leur sont associés soit complètement intégrable, ont leur tenseur de courbure nul.

Si l'on se limite aux éléments plans invariants par $\mathfrak{d}_{x}$ (resp. $\left.\mathfrak{F}_{a}\right)$ c'est-àdire aux droites complexes (resp. paracomplexes), on démontre que les structures hermitiennes (resp. parahermitiennes) pour lesquelles $K$ est indépendant de l'élément plan sans être nul sont les structures isotropes non intégrables (défnies par le théorème 23.1). Ce résultat a êté démontré par BocHNER en sup. posant la structure kahlérienne [2].

On pourrait également definir un scalaire de courbure pour les structures presque hermitiennes d'espèce $k$; les théorèmes 24.1 et 24.2 demeurent valables pour ces structures.

25. Courbure et torsion conformes [38] et [41].

Soit sur une variété $V_{2 n}$ une structure presque hermitienne s déterminée par la donnée d'un champ d'automorphismes $\mathfrak{I}_{x}$ et par une métrique riemannienne $F$ définie positive. Considérons maintenant la famille de structures presque hermitiennes $\boldsymbol{s}_{\lambda}$ déterminées par le champ d'automorphismes $\mathfrak{g}_{x}$ et la forme $F_{\lambda}=\lambda F, \lambda$ étant une fonction différentiable de $x$; si $\Omega$ est la forme extérieure associée à la structure $\mathcal{B}$, à la structure $\mathfrak{s}_{\lambda}$ est associée la forme extérieure $\lambda \Omega$.

De même soit $\mathfrak{g}^{\prime}$ une structure presque parahermitienne déterminée par la donnée d'une forme différentielle extérieure quadratique $\Omega$ et d'un champ de $(n, n)$-involutions $\mathfrak{F}_{x}$ dont le sous-espaces positif et négatif sont intégraux de $\Omega$. On pent considérer les structures $\varepsilon_{\lambda}{ }^{\prime}$ déterminées par la donnée du champ d'automorphismes $\mathfrak{F}_{x}$ et de la forme $\lambda \Omega$.

A chaque structure $\mathfrak{s}_{\lambda}$ (resp. $\mathfrak{s}_{\lambda}{ }^{\prime}$ ) est associée canoniquement une connexion affine, d'où un tenseur de courbure et un tenseur de torsion. Nous allons de plus introduire une courbure et ane torsion conformes respectivement invariante ou multipliee par un scalaire, lorsque l'on multiplie $\Omega$ par $\lambda$.

Comme la donnée de $\mathfrak{d}_{x}$ définit une orientation de $T_{x}$, toute transformation du groupe linéaire complexe $L_{n}^{\prime}$ conserve le signe de $\Omega$ et les structures $\mathfrak{s}_{\lambda}$ correspondent à une valeur positive de $\lambda$. La donnée de $\mathscr{F}_{x}$ ne définit pas une orientation de $T_{x}$, mais l'involution $\mathfrak{F}_{x}$ transformant $\Omega$ en $-\Omega$, il suffit de considérer iUs structures $\mathcal{B}_{\lambda}^{\prime}$ pour lesquelles $\lambda$ est positif. 
Si la structure $\mathfrak{g}$ (resp. $\mathcal{g}^{\prime}$ ) est déterminée localement par $2 n$ formes de PFaff complexes telles que $\omega^{s \prime}=\bar{\omega}^{s}$ (resp. réelles), la forme $\Omega$ s'écrivant $i \Sigma \omega^{s} \wedge \omega^{s \prime}$ (resp. $\Sigma \omega^{s} \wedge \omega^{s^{\prime}}$ ), la structure $\mathfrak{g}_{\lambda}$ (resp. $\mathfrak{s}_{\lambda}^{\prime}$ ) peut être déterminée par les $2 n$ formes de PFAFF: $\tilde{\omega}^{s}=\mu \omega^{s}, \tilde{\omega}^{8 \prime}=\mu \omega^{8 \prime}$ (où $\mu$ est fonction réelle telle que $\mu^{2}=\lambda$ ). Soient $\Omega^{s}, \Omega^{\prime \prime} ; \Gamma^{s}, \Gamma^{s^{\prime}} ; \Omega_{s}^{l}, \Omega_{s^{\prime}}^{l^{\prime}}$ les formes définissant respectivement la première torsion, la deuxième torsion et la courbure de la structure $\mathfrak{s}$ (resp. $\mathfrak{s}^{\prime}$ ) et soient $\tilde{\Omega}^{s}, \tilde{\Omega}^{s^{\prime}} ; \tilde{\Gamma}^{s}, \tilde{\Gamma}^{s^{\prime}} ; \tilde{\Omega}_{s}^{l}, \tilde{\Omega}_{s^{\prime}}^{l}$, les formes definissant les torsions et la courbure de la structure $\mathfrak{s}_{\lambda}$ (resp. $\mathfrak{s}_{\lambda}{ }^{\prime}$ ).

$\frac{d \mu}{\mu}=\theta+\theta^{\prime}$ (où $\theta$ est une forme linéaire en $\omega^{l}$, $\theta^{\prime}$ une forme linéaire en $\left.\omega^{l}\right)$ :

On a: $d \tilde{\omega}^{s}=\frac{d \mu}{\mu} \wedge \tilde{\omega}^{s}+\mu d \omega^{s}, d \tilde{\omega}^{s^{\prime}}=\frac{d \mu}{\mu} \wedge \tilde{\omega}^{s^{\prime}}+\mu d \omega^{s^{\prime}}$, soit en posant

$$
\begin{aligned}
& d \tilde{\omega}^{s}=\Sigma \tilde{\omega}^{l} \wedge \tilde{\omega}_{l}^{s}+\tilde{\Omega}^{s}+\tilde{\Gamma}^{s}, \\
& d \tilde{\omega}^{s^{\prime}}=\Sigma \tilde{\omega}^{l^{\prime}} \wedge \tilde{\omega}_{s^{\prime}}^{l^{\prime}}+\tilde{\Omega}^{s^{\prime}}+\tilde{\Gamma}^{s^{\prime}}, \\
& \tilde{\omega}_{l}^{s}+\tilde{\omega}_{s^{\prime}}^{l^{\prime}}=0,
\end{aligned}
$$

arec :

$$
\begin{array}{ll}
\tilde{\omega}_{l}^{s}=\omega_{l}^{s}+\delta_{l}^{s}\left(\theta-\theta^{\prime}\right) & \tilde{\omega}_{l^{\prime}}^{s^{\prime}}=\omega_{l}^{s^{\prime}}+\delta_{l}^{s}\left(\theta^{\prime}-\theta\right) \\
\tilde{\Omega}^{s}=\mu\left(\Omega s+2 \theta \wedge \omega^{s}\right), & \tilde{\Omega}^{s^{\prime}}=\mu\left(\Omega^{s^{\prime}}+2 \theta^{\prime} \wedge \omega^{s^{\prime}}\right) \\
\tilde{\Gamma}=\mu \Gamma^{s}, & \tilde{\mathbf{\Gamma}}^{s}=\mu \Gamma^{s^{\prime}}, \\
\tilde{\Omega}_{l}^{s}=\Omega_{l}^{s}+2 \delta_{l}^{s} d \theta, & \tilde{\Omega}_{l^{\prime}}^{s^{\prime}}=\Omega_{l^{\prime}}^{s^{\prime}}+2 \delta_{l}^{s} d \theta^{\prime} .
\end{array}
$$

Par suite nous definirons la courbure conforme par les formes

$$
\Pi_{l}^{s}=\Omega_{l}^{s}-\delta_{l}^{s} \frac{\Sigma \Omega_{t}^{t}}{n}, \quad \Pi_{l^{\prime}}^{s^{\prime}}=\Omega_{l}^{s^{\prime}}-\delta_{l}^{s} \frac{\Sigma \Omega_{t^{\prime}}^{t^{\prime}}}{n}
$$

la première torsion conforme par

$$
\Pi^{s}=\Omega^{s}-\frac{\varphi \wedge \omega^{s}}{n-1}, \quad \Pi^{s^{\prime}}=\Omega^{s^{\prime}}-\frac{\varphi^{\prime} \wedge \omega^{s^{\prime}}}{n-1}
$$

où

$$
\varphi=2 \Sigma A_{t_{s}}^{s} \omega^{t}, \quad \varphi^{\prime}=2 A_{t^{\prime} s^{\prime}}^{s^{\prime}} \omega^{t^{\prime}} \quad\left(\text { avec } \quad \Omega^{s}=\Sigma A_{t l}^{s} \omega^{t} \wedge \omega^{l}, \quad \Omega^{s^{\prime}}=\Sigma A_{t^{\prime} \eta^{\prime}}^{s^{\prime \prime}} \wedge \omega^{l^{\prime}}\right) .
$$

La deuxième torsion conforme est par définition identique à la deuxième torsion.

Si $\alpha_{x}$ désigne l'isomorphisme de $T_{x}$ sur son dual défini dans $\S 16$, la forme $\varphi+\varphi^{\prime}$ est égale an produit intérieur $\alpha^{-1}(\Omega) \perp \Sigma\left(\Omega^{s} \wedge \omega^{s^{\prime}}-Q^{s^{\prime}} \wedge \omega^{s}\right)$ ou encore:

$$
\left.\varphi+\varphi^{\prime}=\alpha^{-1}(\Omega) \downarrow \Sigma\left[\left(\Omega^{s}+\Gamma^{s}\right) \wedge \omega^{s^{\prime}}-\left(\Omega^{s^{\prime}}+\Gamma^{v^{\prime}}\right) \wedge \omega^{s}\right]=\alpha^{-1}(\Omega)\right\rfloor d \Omega
$$

La forme $\varphi+\varphi^{\prime}$ est donc la codifférentielle $\tilde{\delta} \Omega$ (of. $\S 17$ ). 
La forme $\Sigma\left(\Pi^{s}+\Gamma^{s}\right) \wedge \omega^{s^{\prime}}-\Sigma\left(\Pi^{s^{*}}+\left[^{s^{\prime}}\right) \wedge \omega^{s}\right.$ est égale a: $d \Omega-\frac{\delta \Omega \wedge \Omega}{n-1}$. Cette forme et la forme $\varphi+\varphi^{\prime}$ sont donc respectivement la forme de torsion conforme et la forme de torsion de la structure presque symplectique a laquelle est subordonnée la structure (resp. $\left.\$^{\prime}\right)$; les deax formes envisagées ne dépendent par conséquent que de la forme $\Omega$ et non de la forme $F$ (resp. $F^{\prime}$ ) échangeable arec $\Omega$.

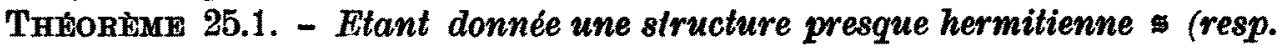
parahermitienne $\left.s^{\prime}\right)$, pour $q u$ 'il existe une fonction $\lambda$ telle que la structure $s_{\lambda}$ (resp. $s_{\lambda}^{\prime}$ ) soit intégrable, il faut et, pour $n>2$, il suffit que la courbure et la torsion conformes soient nulles.

Démonstration: il est évident que les conditions énoncées sont nécessaires en raison même de la définition de la courbure et de la torsion confor. mes. Inversement si $\Pi^{s}=\Gamma^{s}=0, \Pi^{s^{\prime}}=\Gamma^{s^{\prime}}=0$, on a : $\Omega^{s}=\frac{\varphi \wedge \omega^{s}}{n-1}, \Omega^{s^{\prime}}=\frac{\varphi \wedge \omega^{s^{\prime}}}{n-1}$ on $\varphi$ est linéaire en $\omega^{l}$ et $\varphi^{\prime}$ linéaire en $\omega^{l^{\prime}}$; de même si $\Pi_{l}^{z}=0$, $\Pi_{l^{\prime}}^{g^{\prime}}=0$, on a : $\Omega_{l}^{s}=0, \Omega_{l}^{s^{\prime}}=0$ (si $\left.s \neq l\right), \Omega_{l}^{l}=Q_{\mathrm{a}}^{s}, \Omega_{l^{\prime}}^{l^{\prime}}=\Omega_{s^{\prime}}^{z^{\prime}}(l, s=1, \ldots, n)$. Des identités de BrANCHI (22.4), on déduit alors: $\omega^{8} \wedge\left(d \varphi-\Omega_{g}^{8}\right)=0$, $\omega^{s^{\prime}} \wedge\left(d \varphi-\Omega_{g}^{\prime \prime}\right)=0$ $(s=1, \ldots, n)$.

Par suite si $n>2$, on a: $d \varphi=Q_{s}^{s}, d \varphi^{\prime}=\Omega_{s^{\prime}}^{s^{\prime}}$ d'où : $d \varphi+d \varphi^{\prime}=0$; il existe donc une fonction $\mu$ (réelle quelle que soit la nature de la structure) telle que localement $-\frac{d \mu}{\mu}=\frac{\varphi+\varphi^{\prime}}{2(n-1)}$. Les tenseurs de courbare et de torsion de la structure $\varpi_{\lambda}$ (resp. $s_{\lambda}^{\prime}$ ) définie par les formes $\omega^{s}=\mu \omega^{\alpha}, \omega^{\prime \prime}=\mu \omega^{s^{\prime}}$ sont nuls, en raison des relations $(25.2)$, (on suppose $\lambda=\mu^{2}$ ); donc la structure $w_{\lambda}$ (resp. $s_{k}$ ) est intégrable.

On peut d'ailleurs déduire directement de la relation $a \Omega-\frac{\tilde{\varepsilon} \Omega \wedge \Omega}{n-1}$ que $d\left(\varphi+\varphi^{\prime}\right)=d \tilde{\delta} \Omega$ est nul mais le raisonnement indiqué ici est valable ponr des structures qui seront étudiée ultérieurement (\$ 31).

Si $n=2, \Pi^{8}=0$ identiquement, mais il faut imposer, outre la condition $\Pi_{l}^{s}=0$, la condition $d\left(\varphi+\varphi^{\prime}\right)=d \tilde{\delta} \Omega=0$ pour que la structure $\aleph_{\lambda}$ (rtsp. $\star_{\lambda}{ }^{\prime}$ ) soit intégrable, ce qui est en accord avec les résultats de \& 18.

Remarquons que la fonction $\lambda$ introduite dans le thérème $25.1 \mathrm{n}$ ' est définie qu'à un factenr constant près. Si la structure $w_{\lambda}$ (resp. $w_{\lambda}^{\prime}$ ) est intégrable, la structure initiale $s$ (resp. $s^{\prime}$ ) est hermitienne (resp. parahermitienne) et dans le voisinage $U$ d'un point de $V_{2 n}$, on pent trouver un système de coordonnées locales complexes (resp. paracomplexes) $z^{1}, \ldots, z^{n}$ telles que la ferme d'Hermire (resp. forme parahermitienne) associée à la structure (resp. s') puisse s'écrire :

$$
\frac{1}{\lambda\left(z^{1}, \ldots, z^{n}\right)}\left(d z^{i} d \overline{z^{1}}+\ldots+d \varepsilon^{n} d \bar{z}^{n}\right) .
$$


Si la structure $\mathfrak{s}$ (resp. $\mathfrak{s}^{\prime}$ ) est de plus supposée kăhlérienne (resp. parakählérienne), il faut que $\lambda$ soit une constante: la structure $s$ (resp. $\xi^{\prime}$ ) est donc intégrable. Ce résultat a été démontré a'une autre manière par Bochner pour les structures kahlériennes [3].

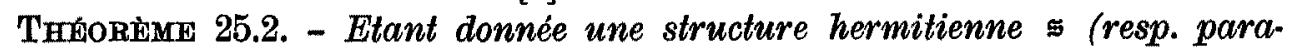
hermitienne $s^{\prime}$ ), pour $q u$ 'il existe une fonction $\lambda$ telle que la structure $\$_{\lambda}$ (resp. $\mathfrak{F}_{\lambda}$ ) soit kählérienne (resp, para-kählérienne), il faut et pour $n>2$ il suffit que la torsion conforme soit nulle.

En effet cette condition réalisée, la forme $\psi=d \Omega-\frac{\tilde{\delta} \Omega \wedge \Omega}{n-1}$ est nulle et la forme $\Omega$ admet un facteur intégrant d'après le théorème 18.1 .

REMARQde: Si la courbure conforme d'une structure presque hermitienne (resp. presque parahermitienne) est nulle, on a : $R_{l l^{\prime h}}^{s}=R_{l t h}^{s}=0, R_{l^{\prime} h^{\prime}}^{s^{\prime}}=0$, $R_{v t^{\prime} h^{\prime}}^{s^{\prime}}=0$ (si $\left.s \neq l\right)$; si de plus la courbure scalaire est nulle, en raison de $(24.2): R_{s t^{\prime} h}^{s}+R_{h^{\prime} s_{s}}^{t}=0$, d' où ; $R_{s t^{\prime} h}^{s}=R_{s^{\prime} t^{\prime} h^{\prime}}^{s^{\prime}}=0$ si $t \neq h ;$ de (24.2) on déduit également $R_{s s^{\prime} s}^{s}=0$; comme $\Omega_{s}^{s}=\Omega_{h}^{h}$, on a aussi : $h_{s h / h}^{s}=0 \quad(s, h=1, \ldots, n)$. On démontre de la même manière que $R_{s t h}^{s}=0, \quad R_{s^{\prime} t^{\prime} h^{\prime}}^{s^{\prime}}=0, \quad R_{s^{\prime} t h}^{s^{\prime}}=0$ $(s, t, h=1, \ldots, n)$. Par suite le tenseur de courbure est nul, d'où la proposition suivante: si la courbure conforme et le scalaire de courbure sont nuls, le tenseur de courbure est nul également. Si de plus la torsion conforme est nulle (dans le cas de. $n=2$ si $d \delta \bar{\Omega}=0$ ) il existe une structure $\approx_{\lambda}$ (resp. $\aleph_{\lambda}^{\prime}$ ) intégrable dont la courbure est par conséquent nulle: $\lambda$ est donc une con. stante; ainsi lorsque la courbure et la torsion conformes sont nulles de même que le scalaire de courbure, la structure correspondante est intégrable, ce qui précise les résultats de [38].

C) Problèmes d'équivalence des struciures presque complexes et presque hermittennes sur une variété à 4 dimensions.

Cette partie du chapitre $\mathrm{V}$ utilise et complète les résultats de [37].

26. Problème d'équivalence des structures presque complexes.

Nous allons appliquer la méthode générale exposée dans le chapitre II à l'étude du problème d'équivalence relatif à des structures presque complexes définies sur des variêtés de dimension 4 . Soit sur une variété $V_{4}$ une structure presque complexe définie par un champ d'automorphismes $\mathfrak{d}_{x}$ de l'espace tangent $T_{x}$. Si l'on suppose la torsion non nulle, on démontrera que l'on peut associer canoniquement à la structure un champ $C$ d'éléments de contact à deux dimensions invariants par la transformation $\mathfrak{J}_{x}$ e'est-à-dire un champ de droites complexes, ce qui n'a pas lieu en général pour $n$ quelconque.

Sur la variété $V_{4}$ la structure presque complexe s peut être déterminée dans le voisinage $U$ d'un point $x$ par la donnée de deux formes de Pfaff complexes linéairement indépendantes $\theta^{4}$ et $\theta^{2}$; les différentielles $d \theta^{1}$ et $d \theta^{2}$ 
peuvent s' ecrire (cf. (21.2)) :

$$
\begin{aligned}
& d \theta^{1}=\theta^{1} \wedge \theta_{1}^{1}+\theta^{2} \wedge \theta_{2}^{1}+A^{1} \bar{\theta}^{1} \wedge \bar{\theta}^{2}, \\
& d \theta^{2}=\theta^{1} \wedge \theta_{1}^{2}+\theta^{2} \wedge \theta_{2}^{2}+A^{2} \bar{\theta}^{1} \wedge \bar{\theta}^{2},
\end{aligned}
$$

où les $\theta_{s}^{l}$ sont des formes de PFaff complexes linéaires en $\theta^{*}, \bar{\theta}^{*}$.

En chaque point, parmi le corepères qui se déduisent du corepère initial par une transformation du groupe lineaire complexe $L_{q}^{\prime}$, en vertu des formules (5.3), on peut choisir une famille $F$ de corepères tels que les composantes du tenseur de torsion soient respectivement égales à 1 et 0 , ce qui détermine une structure $\boldsymbol{s}^{\prime}$ subordonnee a $s$, de groupe structural $G$, sousgroupe de $L_{2}^{\prime}$. Cette structure $\mathfrak{s}^{\prime}$ est déterminée localement par les formes complexes $\omega^{2}$ et $\omega^{2}$ telles que :

$$
\begin{aligned}
& d \omega^{4}=\omega^{1} \wedge \omega_{1}^{1}+\omega^{2} \wedge \omega_{2}^{1}+\bar{\omega}^{1} \wedge \bar{\omega}^{2}, \\
& d \omega^{2}=\omega^{1} \wedge \omega_{1}^{2}+\omega^{2} \wedge \omega_{2}^{2} .
\end{aligned}
$$

La même structure peut être déterminée par les formes de PFafr

$$
\left\{\begin{array}{l}
\pi^{1}=u_{1}^{1} \omega^{1}+u_{2}^{1} \omega^{2} \\
\pi^{2}=u_{2}^{2} \omega^{2}
\end{array}\right.
$$

avec :

$$
u_{2}^{2}=\frac{\bar{u}_{1}^{1}}{u_{1}^{4}} \mathrm{~d}^{\prime} \text { ò̀ : } \quad u_{2}^{2} \bar{u}_{\mathrm{z}}^{2}=1
$$

les $u_{l}^{s}$ étant des fonctions différentiables de $x$ qui en chaque point définissent une transformations de $G^{\prime}$. En raison des relations (26.3) et (26.4), ce groupe $G^{\prime}$, à deux paramètres complexes, laisse invariant un sous-espace de $R^{4}$ à 2 dimensions et une forme quadratique extérieure de rang 2 . A la structure $\mathbf{s}^{\prime}$ est donc associée canoniquement un champ $C$ d'éléments de contact $X_{2}$ a: deux dimensions, défini localement par l'équation: $\omega^{2}=0$; chaque elément de contaot $X_{2}$ est dono invariant par l'automorphisme $\mathfrak{J}_{\infty}$; à la structure est également associée une forme différentielle extérieure de rang 2: $\varphi=\omega^{2} \wedge \bar{\omega}^{2}$.

Remarquons que par différentiation des équations (16.4) on obtient :

$$
\frac{d u_{1}^{1}}{u_{1}^{1}}-\frac{d \bar{u}_{1}^{1}}{\bar{u}_{1}^{1}}+\frac{d u_{2}^{2}}{u_{2}^{2}}=0, \quad \frac{d u_{2}^{2}}{u_{2}^{2}}+\frac{d \bar{u}_{z}^{2}}{\bar{u}_{2}^{2}}=0 .
$$

On a :

$$
\begin{gathered}
d \omega^{2}=\omega^{2} \wedge \omega_{2}^{2}+\omega^{1} \wedge\left(a_{1} \bar{\omega}^{1}+a_{2} \bar{\omega}^{2}\right), \quad \text { soit : } \\
d \pi^{2}=\pi^{2} \wedge\left(\omega_{2}^{2}-\frac{d u_{2}^{2}}{u_{2}^{2}}\right)+a_{1} \frac{u_{2}^{2}}{u_{1}^{1} \bar{u}_{1}^{1}}\left(\pi^{1}-u_{2}^{1} \frac{\pi^{2}}{u_{2}^{2}}\right) \wedge\left(\bar{\pi}^{1}-\bar{u}_{2}^{1} \frac{\bar{\pi}^{2}}{\bar{u}_{2}^{2}}\right)+a_{2} \frac{u_{2}^{2}}{u_{1}^{1} u_{2}^{2}}\left(\pi^{1}-u_{2}^{1} \frac{\pi^{2}}{u_{2}^{2}}\right) \wedge \bar{\pi}^{2} \\
=\pi^{2} \wedge \pi_{2}^{2}+a_{1} \frac{u_{2}^{2}}{u_{1}^{4} \bar{u}_{1}^{1}} \pi^{1} \wedge \bar{\pi}^{1}+\left(-\frac{a_{1} u_{2}^{2}}{u_{1}^{1} \bar{u}_{1}^{1}} \bar{u}_{2}^{1}+\frac{a_{2} u_{2}^{2}}{u_{1}^{1} \bar{u}_{2}^{2}}\right) \pi^{1} \wedge \bar{\pi}^{2}
\end{gathered}
$$


et l'on peut écrire:

$$
d \pi^{2}=\pi^{2} \wedge \pi_{2}^{2}+a_{1}^{\prime} \pi^{1} \wedge \bar{\pi}^{1}+a_{2}^{\prime} \pi^{1} \wedge \bar{\pi}^{2} .
$$

1) $S i d \omega^{2} \wedge \omega^{2} \neq 0$, on peut choisir en chaque point une famille de corepères tels que $a_{1}{ }^{\prime}$ ou $a_{2}^{\prime}$ prenne la valeur 1 .

Si $a_{1} \neq 0$, e' est-à-dire si le champ $C$ n'est pas complètement inlégrable, on choisit en chaque point une famille de corepères tels que $a_{1}{ }^{\prime}=1$ (ce qui détermine $u_{1}^{1} d^{\prime}$ où $u_{2}^{2}$, puis parmi ces corepères, on choisit un corepère distingué tel que $a_{2}^{\prime}=0 ; \mathrm{d}^{\prime}$ où :

$$
\begin{aligned}
& d \pi^{1}=\pi^{1} \wedge\left(h_{1} \bar{\pi}^{1}+h_{2} \bar{\pi}^{2}\right)+\pi^{2} \wedge\left(k_{1} \pi^{1}+l_{1} \bar{\pi}^{1}+l_{2} \bar{\pi}^{2}\right)+\bar{\pi}^{1} \wedge \bar{\pi}^{2} \\
& d \pi^{2}=\pi^{1} \wedge \bar{\pi}^{1}+\pi^{2} \wedge\left(m_{1} \pi^{1}+n_{1} \bar{\pi}^{1}+n_{2} \bar{\pi}^{2}\right) .
\end{aligned}
$$

On obtient en général 8 invariants complexes.

Si $a_{1}=0$, le champ $C$ est complètement intégrable; on choisit une famille de corepères tels que $a_{2}^{\prime}=1$ (ce qui détermine $u_{1}^{1}$ d'où $u_{2}^{2}$ ); on obtient ainsi une structure $\mathfrak{s}^{\prime \prime}$ subordonnée à $\mathfrak{s}^{\prime}, \mathfrak{s}^{\prime \prime}$ pouvant être déterminée également par les formes $\pi^{1}=\omega^{1}+u_{2}^{1} \omega^{2}, \pi^{2}=\omega^{2}$. La forme $d \omega^{2}$ est un invariant pour la structure $\mathfrak{s}^{\prime \prime}$ et l'on peut déterminer en chaque point un corepère distingué tel que: $d \pi^{2}=\pi^{1} \wedge \bar{\pi}^{2}+\pi^{2} \wedge\left(b_{1} \pi^{1}+b_{2} \bar{\pi}^{1}\right)$ ou $b_{1}$ et $b_{2}$ sont des invariants; la différentiation de cette relation montre qu' on a alors:

$$
\begin{aligned}
& d \pi^{1}=\pi^{2} \wedge\left[\left(b_{2}-\bar{b}_{1}\right) \bar{\pi}^{1}+h_{2} \bar{\pi}^{2}\right]+\pi^{2} \wedge\left(k_{1} \pi^{1}+l_{1} \bar{\pi}^{1}+l_{2} \bar{\pi}^{2}\right)+\bar{\pi}^{1} \wedge \bar{\pi}^{2} \\
& d \pi^{2}=\pi^{1} \wedge \bar{\pi}^{2}+\pi^{2} \wedge\left(b_{1} \pi^{2}+b_{2} \bar{\pi}^{1}\right) .
\end{aligned}
$$

On obtient en général 6 invariants complexes.

2) Si $d \omega^{2} \wedge \omega^{2}=0$, aux formes $\omega^{4}$ et $\omega^{2}$ on peut associer de manière unique les formes $\omega_{2}^{2}$ et $\omega_{1}^{1}$ (la forme $\omega_{2}^{1}$ étant définie modulo $\omega^{2}$ ) vérifiant les équations :

$$
\begin{aligned}
& d \omega^{2}=\omega^{2} \wedge \omega_{2}^{2}+a \omega^{1} \wedge \omega^{2}, \\
& d \omega^{1}=\omega^{1} \wedge \omega_{1}^{1}+\omega^{2} \wedge \omega_{2}^{1}+\bar{\omega}^{1} \wedge \overline{\omega^{2}}, \\
& \omega_{2}^{2}+\bar{\omega}_{2}^{2}=0, \quad \omega_{1}^{1}-\overline{\omega_{1}^{1}}+\omega_{2}^{2}=0 .
\end{aligned}
$$

Pour tout autre corepère, on a :

$$
\begin{aligned}
& d \pi^{2}=\pi^{2} \wedge\left(\omega_{2}^{2}-\frac{d u_{2}^{2}}{u_{2}^{2}}\right)+\frac{a}{u_{1}^{1}} \pi^{1} \wedge \pi^{2}, \\
& d \pi^{1}=\pi^{1} \wedge\left(\omega_{1}^{1}-\frac{d u_{1}^{1}}{u_{1}^{1}}\right)+\frac{\pi^{2}}{u_{2}^{2}} \wedge\left(u_{1}^{1} \omega_{2}^{1}-d u_{2}^{1}+u_{2}^{1} \omega_{2}^{2}+a u_{2}^{1} \omega^{1}\right) \bar{\pi}^{1} \wedge \bar{\pi}^{2} ;
\end{aligned}
$$

on pent donc associer aux formes $\pi^{1}$ et $\pi^{2}$ les formes

$$
\pi_{2}^{2}=\omega_{2}^{2}-\frac{d u_{2}^{2}}{u_{2}^{2}}, \quad \pi_{1}^{1}=\omega_{1}^{1}-\frac{d u_{1}^{1}}{u_{1}^{1}}
$$

qui en raison de (26.5) vérifient les relations $\pi_{2}^{2}+\bar{\pi}_{2}^{2}=0, \pi_{1}^{1}-\bar{\pi}_{1}^{4}+\pi_{2}^{2}=0$. 
$\left.1^{\circ}\right)$ Si $a \neq 0$, on choisit $u_{1}^{1}$ et par suite $u_{2}^{2}$ de façon que $\frac{a_{1}}{u_{1}^{1}}=1$. On définit ainsi une structure $\mathfrak{s}^{\prime \prime}$ (subordonnee $a \mathfrak{s}^{\prime}$ ), dont le groupe structural $G^{\prime \prime}$ est a un paramètre complexe, les changements de corepère étant définis par:

$$
\begin{aligned}
& \pi^{\prime 1}=\pi^{1}+u_{2}^{1} \pi^{2}, \\
& \pi^{\prime 2}=\pi^{2} .
\end{aligned}
$$

La forme $\pi_{1}^{1}$ (et par suite $\pi_{2}^{2}$ ) est un invariant pour la structure $\mathfrak{s}^{\prime \prime}$.

Si $\pi_{1}^{1} \wedge \pi^{2} \wedge \bar{\pi}^{z} \neq 0$, on détermine un corepère distingué tel que : $\pi_{1}^{1}=h \pi^{\prime 1}+k \overline{\pi^{\prime 1}}+l \bar{\pi}^{\prime z}$ (ou si $h=0$, tel que $\left.\pi_{1}^{1}=k_{1} \pi^{\prime 1}\right)$; les fonctions $h, k$, $l, k_{1}$ sont alors des invariants.

Si $\pi_{1}^{1}=H \pi^{2}+K \bar{\pi}^{2}$, on peut déterminer un corepère distingué dans le cas où l'une des deux formes $d H \wedge \pi^{2} \wedge \bar{\pi}^{2}$ ou $d K \wedge \pi^{2} \wedge \bar{\pi}^{2}$ est différente de.0. Dans le cas contraire, la structure $\mathcal{s}^{\prime \prime}$ est isotrope; on a alors :

$$
\begin{aligned}
& d \pi^{1}=\pi^{4} \wedge\left(H \pi^{2}+K \overline{\pi^{2}}\right)+\pi^{2} \wedge \pi_{2}^{4}+\bar{\pi}^{1} \wedge \bar{\pi}^{2}, \\
& d \pi^{2}=\pi^{2} \wedge\left[(\bar{K}-H) \pi^{2}+(\bar{H}-K) \bar{\pi}^{2}\right]+\pi^{1} \wedge \pi^{2},
\end{aligned}
$$

(où $\pi_{2}^{t}$ est une forme linéaire en $\pi^{1}, \bar{\pi}^{1}, \bar{\pi}^{2}, \pi^{2}$ et $d u_{2}^{1}$ ) et l'on démontre facilement que le système

$$
\begin{aligned}
& \widehat{\pi^{1}}\left(\widehat{x}, \bar{u}_{2}^{1}, \widehat{d x}, \widehat{d u_{2}^{1}}\right)=\pi^{1}\left(x, u_{2}^{1}, d x, d u_{2}^{1}\right), \\
& \widehat{\pi^{2}}\left(\widehat{x}, \widehat{u_{2}^{1}}, \widehat{d x}, \widehat{d u_{2}^{1}}\right)=\pi^{2}\left(x, u_{2}^{1}, d x, d u_{2}^{1}\right), \\
& \widehat{H}(x)=H(x), \\
& \widehat{K}(x)=K(x)
\end{aligned}
$$

est en involution. Si l'on suppose les données analytiques, en raison de théorème 4.1, le pseudogroupe des automorphismes locaux de la structure est un pseudogroupe de LIE de type infini.

$\left.2^{\circ}\right)$ Si $a=0$, on $a$ :

$$
\begin{aligned}
& d \omega^{1}=\omega^{1} \wedge \omega_{1}^{1}+\omega^{2} \wedge \omega_{2}^{1}+\bar{\omega}^{1} \wedge \bar{\omega}^{2}, \\
& d \omega^{2} \equiv \omega^{2} \wedge \omega_{2}^{2} .
\end{aligned}
$$

En raison des relations (26.9), les formes $d \omega_{1}^{1}$ et $d \omega_{2}^{2}$ sont des invariants pour la structure $s^{\prime}$; la différentiation de (26.11) montre qu'on a alors :

$$
\begin{aligned}
& d \omega_{2}^{2}=\lambda \omega^{2} \wedge \bar{\omega}^{2}, \\
& d \omega_{1}^{1}=i A \omega^{1} \wedge \bar{\omega}^{1}+\left(-\frac{\lambda}{2}+i B\right) \omega^{2} \wedge \bar{\omega}^{2}+B \omega^{1} \wedge \overline{\omega^{2}}+C \overline{\omega^{1}} \wedge \omega^{2},
\end{aligned}
$$

où $\lambda$ est un invariant réel tel que $d \lambda \wedge \omega^{2} \wedge \bar{\omega}^{2}=0$, et $A, B, C$ des fonctions réelles (non invariantes). En particulier lorsque $\lambda=0$, on peut déterminer $\pi^{2}$ à un facteur constant près de façon que $d \pi^{2}=0:$ il existe alors une fonction complexe $f$ telle que localement $\pi^{2}=d f$. 
a) Si $d \omega_{1}^{1} \wedge \omega^{2} \wedge \bar{\omega}^{2} \neq 0$, on peut choisir en chaque point une famille de corepères tels que :

$$
d \pi_{1}^{4}=\varepsilon i \pi^{1} \wedge \bar{\pi}^{1}+\left(-\frac{\lambda}{2}+i h\right) \pi^{2} \wedge \bar{\pi}^{2}
$$

où $\varepsilon= \pm 1$ et $h$ sont des invariants. On obtient ainsi une structure $\mathfrak{s}_{1}^{\prime}$ subordonnée $\grave{a} \mathfrak{s}^{\prime}, \mathfrak{s}_{1}^{\prime}$ pouvant être également déterminée localement par les formes:

$$
\begin{aligned}
& \pi^{\prime 1}=u_{1}^{1} \pi^{1} \\
& \pi^{\prime 2}=u_{2}^{?} \pi^{2}
\end{aligned}
$$

où :

$$
u_{1}^{1} \tilde{u}_{i}^{1}=1, \quad u_{2}^{2}=\frac{\tilde{u}_{i}^{1}}{u_{i}^{4}} .
$$

A la structure $\varepsilon_{1}^{\prime}$ (dont le groupe structural $G_{1}^{\prime}$ est un sous-groupe à un paramètre réel du groupe unitaire $U_{n}$ ) est associée une forme différentielle extérieure invariante : $\pi^{1} \wedge \bar{\pi}^{1}+\pi^{2} \wedge \bar{\pi}^{2}$, ainsi que la forme de PFAFF $\pi_{1}^{1}+\bar{\pi}_{1}^{1}$. On a: $\pi_{1}^{1}+\bar{\pi}_{1}^{1}=A_{1} \pi^{1}+-\bar{A}_{1} \bar{\pi}^{4}+A_{2} \pi^{2}+\bar{A}_{2} \bar{\pi}^{2}$ et comme $d\left(\pi_{1}^{4}+\bar{\pi}_{1}^{4}\right)=2 \varepsilon i \pi^{4} \wedge \bar{\pi}^{1}+$ $+2 i h \pi^{2} \wedge \bar{\pi}^{2}, A_{1} n^{\prime}$ est pas nulle; on peut donc choisir en chaque point un corepère distingué tel que: $\pi_{1}^{4}+\bar{\pi}_{1}^{4}=k_{1}\left(\pi^{\prime 1}+\bar{\pi}^{\prime 1}\right)+k_{2} \pi^{\prime 2}+\overline{k_{2}} \overline{\pi^{\prime 2}}$, où $k_{1}$ et $k_{2}$ sont des invariants $(k$, étant réel, positif).

B) Si $d \omega_{1}^{1} \wedge \omega^{2} \wedge \omega^{2}=0$ et $d \omega_{1}^{1} \wedge \omega^{2} \neq 0$, on $a: A=0, C \neq 0$; on choisit une famille de corepères tels que: $d \pi_{1}^{1}=\pi^{1} \wedge \bar{\pi}^{2}+\bar{\pi}^{1} \wedge \pi^{2}+b \pi^{2} \wedge \bar{\pi}^{2}$ d'où une structure $\mathfrak{s}^{\prime \prime}$ de groupe structural $G^{\prime \prime}, \mathfrak{s}^{\prime \prime}$ pouvant être également déterminée par les formes $\pi^{\prime 1}=\pi^{1}+u_{2}^{1} \pi^{2}, \pi^{\prime 2}=\pi^{2}$. La forme $\pi_{1}^{1}$ devient une forme invariante et l'on peut déterminer un corepère distingué tel que: $\pi_{1}^{4}=k_{1} \pi^{\prime 1}+$ $+k_{2} \pi^{\prime 2}+k_{3} \bar{\pi}^{\prime 1}$ (si $k_{3}=0$, tel que $\pi_{1}^{1}=k_{1} \pi^{\prime 2}+k_{4} \bar{\pi}^{\prime 2}$, si $\pi_{1}^{1}=l \pi^{\prime 2}+\bar{l}^{\prime} \bar{\pi}^{\prime 2}$ tel que $\left.d l=\pi^{\prime 1}+m \pi^{\prime 2}\right)$.

ү) Si $d \omega_{1}^{1}=\left(-\frac{\lambda}{2}+i h\right) \omega^{2} \wedge \bar{\omega}^{2}$, où $h$ est un invariant tel que $d h \wedge \omega^{2} \wedge \bar{\omega}^{2}$ on peut distinguer encore 4 cas :

1. Si l'un des invariants $\lambda$ ou $h$ au moins $n$ 'est pas une constante, on choisit $\pi^{2}$ de façon. que $d \lambda$ où $d h$ soit égal à $\rho\left(\pi^{2}+\bar{\pi}^{2}\right)$ où $\rho$ est un invariant réel positif; on definit ainsi une structure $\mathfrak{s}_{2}^{\prime}$ de groupe structural $G_{2}{ }^{\prime}$ qui peut être également déterminée localement par les formes

$$
\pi^{\prime 1}=u_{1}^{1} \pi^{1}+u_{2}^{1} \pi^{2}, \quad \pi^{\prime 2}=\pi^{2}
$$

où $u_{1}^{1}$ est réel; la forme $\pi_{2}^{2}$ est alors invariante et si $\pi_{2}^{2} \wedge \pi^{2} \wedge \bar{\pi}^{2} \neq 0$, on peut choisir en chaque point un corepère distingué tel que : $\pi_{2}^{2}=k \pi^{1}-\vec{k} \pi^{1}$ (où $k \bar{k}=1$ ).

Si $\pi_{2}^{2}=\mu \pi^{2}-\bar{\mu} \bar{\pi}^{2}$ (où $\mu$ est un invariant tel qùe $d \mu \wedge \pi^{2} \wedge \ddot{\pi}^{2}=0$ ), la structure $\mathfrak{s}_{2}^{\prime}$ est isotrope. On obtient ləs equations :

$$
\begin{aligned}
& d \pi^{1}=\pi^{1} \wedge \pi_{1}^{4}+\pi^{2} \wedge \pi_{2}^{1}+\bar{\pi}^{1} \wedge \bar{\pi}^{2} \\
& d \pi^{2}=-\bar{\mu} \pi^{2} \wedge \bar{\pi}^{2},
\end{aligned}
$$


où $\pi_{1}^{4}$ est une forme linéaire en $\pi^{4}, \pi^{2}, \bar{\pi}^{1}, \bar{\pi}^{2}$ et $d u_{1}^{4}$, la forme $\pi_{z}^{1}$ étant linéaire en $\pi^{1}, \pi^{2}, \overline{\pi^{1}}, \overline{\pi^{2}}, d u_{2}^{1}$ (telle que le coefficient de $\pi^{2}$ depend d' un parametre $v$ ). Le système $\widehat{\pi^{4}}=\pi^{1}, \widehat{\pi^{2}}=\pi^{2}, \widehat{\mu}=\mu, \widehat{h}=h$ n' étant pas en involution, on le prolonge et l'on obtient le système en involution: $\widehat{\pi^{1}}=\pi^{1}, \widehat{\pi^{2}}=\pi^{2}, \widehat{\pi_{1}^{1}}=\pi_{1}^{4}$, $\frac{\pi_{2}^{1}}{2}=\pi_{2}^{1}, \widehat{h}=h, \widehat{\mu}=\mu$ car on a :

$$
\begin{aligned}
& d \pi^{1}=\pi^{1} \wedge \pi_{1}^{1}+\pi^{2} \wedge \pi_{2}^{1}+\bar{\pi}^{1} \wedge \bar{\pi}^{2} \\
& d \pi^{2}=-\bar{\mu} \pi^{2} \wedge \bar{\pi}^{2} \\
& d \pi_{1}^{1}=(-\overline{2}+i h) \pi^{2} \wedge \bar{\pi}^{2} \\
& d \pi_{2}^{1}=\pi_{2}^{1} \wedge\left(\pi_{1}^{1}-\bar{\mu} \bar{\pi}^{2}\right)+\left(-\frac{\lambda}{2}+i h+1\right) \pi^{1} \wedge \bar{\pi}^{2}+\theta \wedge \pi^{2},
\end{aligned}
$$

où $\theta$ est une forme linéaire en $\pi^{1}, \pi^{2}, \bar{\pi}^{4}, \pi^{2}, d u_{1}^{4}, d u_{2}^{1}, d v$ (définie modulo $\pi^{2}$ ). Supposons désormais les données analytiques: en raison de l'étude faite dans $\$ 4$, le pseudogroupe des automorphismes locaux de la structure considérée est un pseudogroupe de Lie au sens large.

2. $S i \lambda$ et $h$ sont des constantes, $\lambda n^{\prime}$ étant pas nulle, la structure $\mathfrak{g}^{\prime}$ est isotrope; on a les équations :

$$
\begin{aligned}
& d \pi_{1}^{4}=\pi^{4} \wedge \pi_{1}^{4}+\pi^{2} \wedge \pi_{2}^{4}+\bar{\pi}^{4} \wedge \bar{\pi}^{2}, \\
& d \pi^{2}=-\pi^{2} \wedge\left(\pi_{1}^{4}-\overline{\pi_{1}^{4}}\right) \text {, } \\
& d \pi_{1}^{1}=\left(-\frac{\lambda}{2}+i \hbar\right) \pi^{2} \wedge \bar{\pi}^{2} \\
& d \pi_{2}^{4}=\pi_{2}^{1} \wedge\left(2 \pi_{1}^{1}-\bar{\pi}_{1}^{4}\right)+\left(-\frac{\lambda}{2}+i h+1\right) \pi^{4} \wedge \bar{\pi}^{2}+\theta \wedge \pi^{2} .
\end{aligned}
$$

Le système prolonge $\widehat{\pi^{4}}=\pi^{4}, \widehat{\pi^{2}}=\pi^{2}, \widehat{\pi_{1}^{i}}=\pi_{1}^{1}, \widehat{\pi_{2}^{1}}=\pi_{2}^{1}$ est en involution: on a encore un pseudogroupe de LIE au sens large.

3. Si $\lambda=0$ et si h est une constante non nulle, on peut déterminer la forme $\pi^{2}$ (à un facteur constant près) de façon que $d \pi^{2}=0$. On obtient ainsi une structure $\mathfrak{g}_{2}^{\prime \prime}$ (subordonnée à $\mathfrak{s}^{\prime}$ ) qui est isotrope et dont le pseudogroupe des automorphismes locaux est également un pseudogroupe de LIF au sens large; on a en effet:

$$
\begin{aligned}
& d \pi^{1}=\pi^{1} \wedge \pi_{1}^{1}+\pi^{2} \wedge \pi_{2}^{1}+\bar{\pi}^{1} \wedge \bar{\pi}^{2}, \\
& d \pi^{2}=0 \\
& d \pi_{1}^{1}=i h \pi^{2} \wedge \bar{\pi}^{2}, \\
& d \pi_{2}^{1}=\pi_{2}^{1} \wedge \pi_{1}^{1}+(i h+1) \pi^{1} \wedge \bar{\pi}^{2}+\theta \wedge \pi^{2},
\end{aligned}
$$

et le système prolongé $\widehat{\pi}^{1}=\pi^{1}, \widehat{\pi^{2}}=\pi^{2}, \widehat{\pi_{1}^{1}}=\pi_{1}^{1}, \widehat{\pi_{2}^{4}}=\pi_{2}^{1}$ est en involution.

4. Si $\lambda=h=0$, on peut déterminer $u_{1}^{1}$ à un facteur constant près (d'où $\pi^{2}$ ) de façon que $\pi_{\mathfrak{l}}^{4}=0$. On définit ainsi une structure $\mathfrak{g}^{\prime \prime}$ de groupe 
structural $G$ (of. cas $1^{\circ}$ ). Cette structure est isotrope et localement homogène car on a les équations :

$$
\begin{aligned}
& d \pi^{1}=\pi^{2} \wedge \pi_{2}^{1}+\bar{\pi}^{1} \wedge \bar{\pi}^{2}, \\
& d \pi^{2}=0,
\end{aligned}
$$

où $\pi_{2}^{1}$ est une forme linéaire en $\pi^{1}, \pi^{2}, \bar{\pi}^{1}, \bar{\pi}^{2}$, et $d u_{2}^{1}$. L.e système $\widehat{\pi^{1}}=\widehat{\pi^{1}}$, $\widehat{\pi^{2}}=\pi^{2}$ etant en involution, le pseudogroupe des automorphismes locaux de la structure est un pseudogroupe de Lie de type infini.

L'étude faite dans $\$ 26$ pent se résumer de la manière suivante : étant

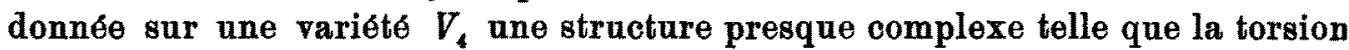
ne soit pas nulle, on peut en général déterminer canoniquement on chaque point un corèpere distingué (notamment lorsque le champ $C$ associé a la structure n'est pas completement intégrable); dans le cas contraire, le psendogroupe des automorphismes locaux de la structure est un psendogroupe de LIE de type infini on un pseudogroupe de LIE au sens large.

\section{7. - Problème d'équivalence des structures presque hermitiennes.}

Soit sur une variêté $V_{\text {a }}$ une structure presque hermitienne $S$ telle que la deuxième torsion de la connexion hermitienne associée a la structure ne soit pas nulle. Cette structure $S$ peut être definie dans le voisinage $U$ d'un point de $V_{4}$ par deux formes de PFafr complexes $\theta^{1}$ et $\theta^{2}$ telles que la métrique hermitienne et la forme extérieure $\Omega$ correspondante s' écrivent respectivement: $d s^{2}=\theta^{1} \bar{\theta}^{1}+\theta^{2} \bar{\theta}^{2}$ et $\Omega=i\left(\theta^{1} \wedge \bar{\theta}^{1}+\theta^{2} \wedge \bar{\theta}^{2}\right)$.

Les différentielles $d \theta^{1}$ et $d \theta^{2}$ vérifient les équations (cf. (22.3) ):

$$
\begin{aligned}
& d \theta^{1}=\theta^{1} \wedge \theta_{1}^{1}+\theta^{2} \wedge \theta_{2}^{1}+\beta^{1} \theta^{1} \wedge \theta^{2}+\alpha^{1} \bar{\theta}^{1} \wedge \bar{\theta}^{2}, \\
& d \theta^{2}=\theta^{1} \wedge \theta_{1}^{2}+\theta^{2} \wedge \theta_{2}^{2}+\beta^{2} \theta^{1} \wedge \theta^{2}+\alpha^{2} \bar{\theta}^{1} \wedge \bar{\theta}^{2}, \\
& \theta_{1}^{1}+\bar{\theta}_{1}^{1}=0, \quad \theta_{2}^{2}+\bar{\theta}_{2}^{2}=0, \quad \theta_{2}^{1}+\bar{\theta}_{1}^{z}=0 .
\end{aligned}
$$

En chaque point on peut choisir une famille. $F$ de corepères unitaires tels $q u e$ les composantes du tenseur définissant la deuxième torsion soient respectivement un nombre réel positif et 0 ; à cette famille $F$ est associée une structure $S^{\prime}$ subordonnée a $S$ (de groupe structural $g$ sous-groupe de $U_{n}$ ) déterminée localement par les formes complexes $\omega^{1}$ et $\omega^{2}$ telles que:

$$
\begin{aligned}
& d \omega^{1}=\omega^{1} \wedge \omega_{1}^{1}+\omega^{2} \wedge \omega_{2}^{4}+C^{1} \omega^{1} \wedge \omega^{2}+A \bar{\omega}^{1} \wedge \bar{\omega}^{2}, \\
& d \omega^{2}=\omega^{1} \wedge \omega_{2}^{1}+\omega^{2} \wedge \omega_{2}^{2}+C^{2} \omega^{1} \wedge \omega^{2}, \\
& \omega_{1}^{1}+\bar{\omega}_{1}^{1}=0, \quad \omega_{2}^{2}+\bar{\omega}_{2}^{2}=0, \quad \omega_{1}^{2}+\bar{\omega}_{2}^{4}=0,
\end{aligned}
$$

où $A$ est un invariant réel positif; la même structure peut être déterminée localement par les formes

$$
\begin{aligned}
& \pi^{1}=u_{1}^{1} \omega^{1}, \\
& \pi^{z}=u_{2}^{2} \omega^{2},
\end{aligned}
$$


où

$$
u_{1}^{1} \bar{u}_{1}^{1}=1, \quad u_{2}^{2} \bar{u}_{2}^{2}=1, \quad \frac{u_{1}^{1}}{\bar{u}_{1}^{1} \bar{u}_{2}^{2}}=1, \quad \text { soit : } \quad u_{2}^{2}=\frac{1}{\left(u_{1}^{1}\right)^{2}}
$$

$u_{1}^{1}$ et $u_{2}^{2}$ déterminent en chaque point une transformation de $g$ lequel est dono à un paramètre réel; $g$ laisse invariants deux-sous-espaces supplémentaires à deux dimensions de $R^{4}$. Par suite à la structure $S^{\prime}$ sont associés deux champs $C_{1}$ et $C_{2}$ d'éléments de contact supplémentaires $X_{1}$ et $X_{2}$ à deux dimensions (définis respectivement par $\omega^{1}=0, \omega^{2}=0$ ); $X_{1}$ et $X_{2}$ sont invariantes par l'automorphisme $\mathfrak{J}_{x}$; donc (cf. $\S 12$ ) à la structure $S^{\prime}$ sont égale. ment associés un champ d'involutions $\mathfrak{J}_{x}$ telles que $\mathfrak{J}_{\alpha} \mathfrak{F}_{\infty}=\mathfrak{J}_{x} \mathfrak{J}_{x}$ et un champ d'automorphismes $\mathfrak{H}_{x}=\mathfrak{J}_{x} \mathfrak{F}_{x}$. Il en résulte que la structure $S^{\prime}$ est également subordonnée à une structure infinitésimale régulière de groupe structural isomorphe à $L_{1}^{\prime} \times L_{1}^{\prime}$ (cf. §. 33).

Aux formes $\pi^{1}$ et $\pi^{2}$ sont associées les formes $\pi_{1}^{1}, \pi_{2}^{2}, \pi_{2}^{1}, \pi_{1}^{2}$ telles que:

$$
\begin{array}{ll}
\pi_{1}^{1}=\omega_{1}^{1}-\frac{d u_{1}^{1}}{u_{1}^{1}}, & \pi_{1}^{2}=\frac{u_{2}^{2}}{u_{1}^{4}} \omega_{1}^{2}, \\
\pi_{2}^{2}=\omega_{2}^{2}-\frac{d u_{2}^{2}}{u_{2}^{2}}, & \pi_{2}^{1}=\frac{u_{1}^{1}}{u_{2}^{2}} \omega_{2}^{1} .
\end{array}
$$

Par différentiation des équations (27.3), on obtient: $2 \frac{d u_{1}^{1}}{u_{1}^{1}}+\frac{d u_{2}^{2}}{u_{2}^{2}}=0$ et par suite:

$$
2 \pi_{1}^{4}+\pi_{2}^{2}=2 \omega_{1}^{1}+\omega_{2}^{2} .
$$

La forme $2 \omega_{1}^{1}+\omega_{2}^{2}$ est donc un invariant pour la structure $S^{\prime}$. Sont également invariantes les formes:

$$
\begin{gathered}
d A, \quad \omega^{1} \wedge \bar{\omega}^{1}, \quad \omega^{2} \wedge \bar{\omega}^{2}, \quad \omega_{1}^{2} \wedge \omega_{2}^{1}, \quad d \omega_{1}^{1}, \quad d \omega_{2}^{2}, \quad \Omega_{1}^{1}=d \omega_{1}^{1}-\omega_{1}^{2} \wedge \omega_{2}^{1}, \\
\Omega_{2}^{2}=d \omega_{2}^{2}-\omega_{2}^{1} \wedge \omega_{1}^{2}, \quad \Omega_{1}^{2} \wedge \Omega_{2}^{1} .
\end{gathered}
$$

Remarquons que les formes $\Omega_{1}^{1}, \Omega_{2}^{2}, \Omega_{2}^{1}, \Omega_{2}^{1}$ definissent la courbure de la connexion hermitienne.

D'après un théorème de LEPAGE (§ 15), aux formes $d \Omega, i d\left(\omega^{1} \wedge \bar{\omega}^{1}\right)$, $i d\left(\omega^{2} \wedge \overline{\omega^{2}}\right), \Omega_{2}^{2}$ qui sont de degré 3 , correspondent les formes de PFAFF bien déterminées $\Theta, \Theta_{1}, \Theta_{2}, \Theta_{3}$ telles que :

$$
\begin{gathered}
d \Omega=\Omega \wedge \Theta, i d\left(\omega^{1} \wedge \bar{\omega}^{1}\right)=\Omega \wedge \Theta_{1}, i d\left(\omega^{2} \wedge \bar{\omega}^{2}\right)=\Omega \wedge \Theta_{2}, i d \Omega_{2}^{2}=-i d \Omega_{1}^{1}=\Omega \wedge \Theta_{3} \\
\text { (on } \left.a: \Theta=\Theta_{1}+\Theta_{2}\right) .
\end{gathered}
$$

La condition nécessaire et suffisante pour que la structure soit subordonnée à une structure presque kählérienne est que $\Theta=0$.

Les formes $\Omega$, $i \omega^{1} \wedge \bar{\omega}^{1}, i \omega^{2} \wedge \bar{\omega}^{2}, i \omega_{1}^{2} \wedge \omega_{2}^{1}$ etant réelles, les formes $\Theta, \Theta_{1}, \Theta_{2}, \Theta_{3}$ sont réelles également ainsi que $d A$ et $i\left(2 \omega_{1}^{4}+\omega_{2}^{2}\right) ;$ l'une d'elles peut s' écrire: $\lambda_{1} \omega^{1}+\bar{\lambda}_{1} \bar{\omega}^{4}+\lambda_{2} \omega^{2}+\bar{\lambda}_{2} \bar{\omega}^{2}$. 
$\left.1^{\circ}\right)$ Si l' une au moins des formes de Pfaff invariantes n'est pas nulle, on pent déterminer en chaque point un corepère distingué tel que l'une de ces formes s'ecrive $k_{1}\left(\pi^{1}+\bar{\pi}^{1}\right)+h \pi^{2}+\bar{h} \bar{\pi}^{2}, k_{1}$ et $h$ étant des invariants ( $k_{1}$ est réel positif). Si $\lambda_{1}=0$, on choisit la forme $\pi^{2}$ de façon que la forme invariante soit égale à $k_{2}\left(\pi^{2}+\bar{\pi}^{2}\right)$, $k_{2}$ stant un invariant réel positif. La forme $\pi^{i}$ est alors définie au signe près.

20) Si toutes les formes de Pfaff invariantes sont nulles, on a alors une structure subordonnée à une structure presque kahlérienne et les deux champs $C_{1}$ et $C_{2}$ sont de plus complètement intégrables. Dans ce cas comme $d\left(\omega^{1} \wedge \bar{\omega}^{1}\right)=0$, la forme $\omega_{2}^{4}$ peut s'écrire $a_{1} \bar{\omega}^{1}+a_{2} \omega^{2}$ et l'on $a$ :

$$
\pi_{2}^{1}=\frac{u_{1}^{4}}{u_{2}^{2}}\left(\frac{a_{1}}{\bar{u}_{1}^{1}} \bar{\pi}^{1}+\frac{a_{2}}{u_{2}^{2}} \pi^{2}\right)=a_{1}\left(u_{1}^{1}\right)^{4} \bar{\pi}^{1}+a_{2}\left(u_{1}^{1}\right)^{3} \pi^{2}=a_{1}^{\prime} \bar{\pi}^{4}+a_{2}^{\prime} \pi^{2} .
$$

a) Si la forme $\omega_{2}^{4} n^{\prime}$ est pas nulle, on détermine en chaque point un corepère distingué tel que $a_{1}^{\prime}$ soit réel positif (on a 4 déterminations pour $u_{i}^{\prime}$ ) ou si $a_{1}=0$, tel que $a_{2}^{1}$ soit réel positif (5 déterminations pour $u_{1}^{i}$ ).

B) Si la forme $\omega_{2}^{1}$ est nulle, la structure $S^{\prime}$ est isotrope et localement homogène. On a en effet:

$$
d \pi^{1}=\pi^{1} \wedge \pi_{1}^{1}+A \bar{\pi}^{1} \wedge \bar{\pi}^{2}, \quad d \pi^{2}=-2 \pi^{2} \wedge \pi_{i}^{4},
$$

où $A$ est une constante positive; la différentiation de ces equations montre que: $d \pi_{1}^{4}=n \pi^{2} \wedge \bar{\pi}^{2}$, avec $n=A^{2}$. Nous obtenons donc en definitive les équations :

$$
\begin{aligned}
& d \pi^{4}=\pi^{1} \wedge \pi_{1}^{4}+A \bar{\pi}^{1} \wedge \bar{\pi}^{2} \\
& d \pi^{2}=-2 \pi^{2} \wedge \pi_{1}^{1}, \\
& d \pi_{1}^{1}=A^{2} \pi^{2} \wedge \bar{\pi}^{2}
\end{aligned}
$$

ou $\pi_{1}^{1}$ 'est une forme linéaire en $\pi^{4}, \bar{\pi}^{1}, \pi^{2}, \bar{\pi}^{2}, d u_{1}^{1}$ : les formes $\pi^{1}, \pi^{2}, \pi_{1}^{1}$ sont définies sur la variété des corepères déterminant la structure $S^{\prime}$ (cette variété est à 5 dimensions réelles). Les équations (27.8) sont les équations de structure d'un groupe de Lie à 5 paramètres réels; on en déduit que le pseudogroupe des automorphismes locaux de la structure est équivalent à un psendogroupe de Lie à 5 paramètres.

Si l'on pose : $\pi^{4}=\theta^{1}+i \theta^{2}, \pi^{2}=\theta^{3}+i \theta^{4}, \pi_{1}^{4}=-\frac{i}{2} \theta^{5}\left(\theta^{4}, \theta^{2}, \theta^{3}, \theta^{4}, \theta^{5}\right.$ étant des formes rélles), on obtient:

$$
\begin{aligned}
& d \theta^{4}=\frac{1}{2} \theta^{2} \wedge \theta^{5}+A\left(\theta^{4} \wedge \theta^{3}-\theta^{2} \wedge \theta^{4}\right) \\
& d \theta^{2}=-\frac{1}{2} \theta^{1} \wedge \theta^{5}-A\left(\theta^{4} \wedge \theta^{4}+\theta^{2} \wedge \theta^{3}\right) \\
& d \theta^{3}=-\theta^{4} \wedge \theta^{5} \\
& d \theta^{4}=\theta^{3} \wedge \theta^{5} \\
& d \theta^{5}=4 A^{2} \theta^{3} \wedge \theta^{4}
\end{aligned}
$$


Le groupe de LIE admet un sous-groupe distingué à 2 paramètres (défini par $\theta^{3}=\theta^{4}=\theta^{5}=0$ ) isomorphe au groupe des translations du plan, le groupe quotient étant isomorphe au groupe des rotations à 3 paramètres.

Considérons à nouveau la variété $V_{4}$; sur les variétés intégrales du champ $C_{1}$ (défini par $\theta^{1}=\theta^{2}=0$ ), a la métrique $\left(\theta^{3}\right)^{2}+\left(\theta^{4}\right)^{2}$ est associée une connexion riemannienne à courbure constante négative (égale à $-4 A^{2}$ ). Sur les variétés intégrales du champ $C_{2}$ (défini par $\theta^{3}=\theta^{*}=0$ ), à la métrique $\left(\theta^{1}\right)^{2}+\left(\theta^{2}\right)^{2}$ est associée une connexion riemannienne à courbure nulle; ces variétés intégrales sont munies d'une structure d'espace localement euclidien.

Les résultats de $\$ 7$ peuvent se résumer de la manière suivante: étant donnée sur une variété $V_{4}$ une structure presque hermitienne, on peut en général déterminer canoniquement en chaque point un corepère unitaire distingué (notamment lorsqùe la structure n'est pas presque kahlérienne); dans le cas contraire la structure admet une structure subordonnée isotrope et localement homogène dont le groupe structural est à un paramètre réel. Ces résultats sont conformes au théorème 6.2 puisque l'on peut associer canoniquement une connexion affine à une structure presque hermitienne.

28. Remarques concernant le problème d'équivalenee des structures presque paracomplexes et presque parahermitiennes.

Sur une variété $V_{4}$ à une structure presque paracomplexe déterminée par la donnée de deux champs $\mathfrak{C}_{1}$ et $\mathfrak{C}_{2}$ non complètement intégrables d'éléments plans supplémentaires $P_{1}$ et $P_{2}$, on peut associer canoniquement un troisième champ $C$ d'éléments plans $P$ tels $q u^{\prime}$ 'en chaque point $x$ de $V_{4}$, $P$ coupe respectivement $P_{1}$ et $P_{2}$ suivant une droite: le champ $\mathcal{C}$ est un champ de droites paracomplexes. Le problème d'équivalence se traite par les mêmes méthodes que pour les structures presque complexes mais est conduit à envisager un nombre de cas supérieur.

Si l'on suppose de plus la structure presque parahermitienne (les champs $\mathfrak{C}_{1}$ et $\mathcal{C}_{2}$ étant toujours supposés non complètement intégrables), à cette structure $S$ sont associés canoniquement deux champs $C$ et $C^{\prime}$ d'éléments plans supplémentaires $P$ et $P^{\prime}$ coupant respectivement l'élément plan $P_{1}$ (resp. $P_{2}$ ) suivant les droites $D_{1}$ et $D_{1}^{\prime}$ (resp. $D_{2}$ et $D_{2}^{\prime}$ ); par suite à la structure $S$ sont associés trois champs de $(1,1)$-involutions $\mathfrak{J}_{x}, x_{x} \mathfrak{F}$, $\mathfrak{H}_{x}$ telles que $\mathfrak{J}_{x} \mathfrak{F}_{x}=\mathscr{F}_{x} \mathfrak{J}_{x}=\mathfrak{H}_{x}$ (cf. $\S 12$ ); on peut déterminer sur $V_{4}$ une structure $S^{\prime}$ subordonnée à $S$, dont le groupe structural est à un paramètre réel, structure également subordonnée à une structure infinitésimale régulière dont le groupe structural est isomorphe à $L_{1} \times L_{1} \times L_{1} \times L_{1}$ (cf. $\S 33$ ).

Comme pour les structures presque hermitiennes, on peut en général déterminer canoniquement en chaque point un corepère para-unitaire (notam. ment lorsque la structure $S$ n'est pas presque parakahlérienne); dans le cas contraire, la structure $S^{\prime}$ qui est alors isotrope et localement homogène peut 
être déterminée localement par les formes réelles $\omega^{1}, \omega^{2}, \omega^{3}$, $\omega^{4}$ ou par les formes $\pi^{4}=u_{1}^{4} \omega^{4}, \pi^{2}=u_{2}^{2} \omega^{2}, \pi^{3}=u_{3}^{3} \omega^{3}, \pi^{4}=u_{4}^{4} \omega^{4}\left(\right.$ ò̀ $\left.u_{1}^{4} u_{3}^{3}=1, u_{2}^{2} u_{4}^{4}=1, u_{2}^{2}=\frac{1}{\left(u_{1}^{1}\right)^{2}}\right)$ telles que les champs $\mathfrak{C}_{1}, \mathfrak{C}_{2}, C, C^{\prime}$ soient respectivement definis par: $\pi^{3}=\pi^{4}=0, \pi^{1}=\pi^{2}=0, \pi^{2}=\pi^{4}=0, \pi^{1}=\pi^{3}=0$.

Les différentielles $d \pi^{*}$ vérifient les équations :

$$
\begin{aligned}
& d \pi^{4}=\pi^{1} \wedge \pi_{1}^{1}+\pi^{3} \wedge \pi^{4}, \\
& d \pi^{2}=-2 \pi^{2} \wedge \pi_{1}^{4}, \\
& d \pi^{3}=-\pi^{3} \wedge \pi_{1}^{4}+n \pi^{4} \wedge \pi^{2}, \\
& d \pi^{4}=2 \pi^{4} \wedge \pi_{1}^{1} \\
& d \pi_{1}^{4}=n \pi^{2} \wedge \pi^{4},
\end{aligned}
$$

où $n$ est une constante, la forme $\pi_{1}^{4}$ étant une forme linéaire en $\pi^{1}, \pi^{2}, \pi^{3}, \pi^{4}$ et $d u_{1}^{1}:$ l' ensemble des formes $\pi^{2}, \pi^{2}, \pi^{3}, \pi^{4}, \pi_{1}^{4}$ est définie sur la variété des repères (à 5 dimensions).

Le pseudogroupe des automorphismes locaux de cette structure est loca. lement équivalent à un groupe de LIE à 5 paramètres.

\section{Chapitae VI.}

\section{Structures presque quaternioniennes de deuxlème espèce.}

Les résultats obtenus dans ce chapitre ont été exposés en partie dans [39]. Comme dans le chapitre V, les indices latins varient de 1 a $n$ et, sauf mention spéciale, les indices grecs de 1 a $2 n$, aveo $s^{\prime}=s+n$. Par contre nous $n^{\prime}$ adopterons pas la notation $\omega^{\prime \prime}$ pour l'imaginaire conjuguée d'une forme complexe ou paracomplexe $\omega^{*}$.

\section{Génóralités.}

Nous étudierons dans ce chapitre des structures infinitésimales régulières I définies sur une variéte $V_{2 n}, r$ fois différentiable $(r \geq 3)$, de groupe structural $\widehat{L}_{n}$ (of. $\left.\S 13\right)$. Si $n$ est pair $(n=2 p)$, la structure $\mathfrak{F}$ sera dite presque quaternionienne de deuxième espèce (de groupe structural $\stackrel{\circ}{\prime \prime}_{p}$ ). Les structures presque quaternioniennes, dont le groupe structural est le groupe lineaire quaternionien $L^{\prime \prime}$, ont été définies par C. Ehresmans [24].

Il résulte des propriétés étudiées dans $\$ 13$ :

THóonìme 29.1. - Sur une variété $V_{2 n}$ une structure infinitésimale régulière, de groupe structural $\widehat{L}_{n}$ est déterminée par la donnée d'un triplet $\left(C_{1}, C_{2}, C_{3}\right)$ de champs deux fois différentiables de n-éléments de contact $X_{n}$, $X_{n}^{\prime}, Y_{n}$ deux à deux supplémentaires ou par la donnée de l'un des couples $(\mathfrak{I}, \mathfrak{J}),(\mathfrak{J}, \mathfrak{H}),(\mathfrak{F}, \mathfrak{X})$ de champs d'automorphismes $\mathfrak{J}_{\mathfrak{x}}, \mathfrak{J}_{\mathfrak{x}}, \mathfrak{J}_{x}$ de l'espace 
tangent $T_{\infty} \grave{a} V_{2 n}$ au point $x \in V_{2 n}$ tels que $\left(\mathscr{J}_{x}\right)^{2}=-1,\left(\mathscr{J}_{x}\right)^{2}=1,\left(\mathcal{H}_{x}\right)^{2}=1$, $\mathfrak{J}_{x} \mathfrak{F}_{\infty}=-\mathfrak{J}_{x} \mathfrak{J}_{x}=\mathfrak{H}_{x}$ ou éncore par la donnée d'un champ d'automorphismes $\mathfrak{J}_{x}$ et d' un champ de n-éléments de contact réels.

A la structure est associée un quatrième champ $C_{4}$ de n-éléments $Y_{n}^{\prime}=\mathfrak{J}_{x} Y_{n}$.

La même structure $\mathfrak{I}$ peut être déterminée par tout triplet $\left[C^{\left(\mu_{1}\right)}, C^{\left(\mu_{2}\right)}, C^{\left(\mu_{2}\right)}\right]$ de champs dépendant de trois constantes arbitraires $\mu_{1}, \mu_{2}, \mu_{3}$; mais tout triplet $\left[C^{\left(\mu_{1}\right)}, C^{\left(\mu_{2}\right)}, C^{\left(\mu_{3}\right)}\right]$ de champs dépendant de trois fonction arbitraires $\mu_{1}, \mu_{2}, \mu_{3}$ détermine une famille $\boldsymbol{\Xi}_{\mu_{1} \mu_{2} \mu_{3}}$ de structures de même espèce que la structure $\mathfrak{g}$ mais non la mème structure $\left({ }^{\circ}\right)$.

\section{Connexions affines associées à une structure $\mathbf{S}$.}

Dans le voisinage $U$ d'un point de $V_{2 n}$ une structure $\mathfrak{g}$ peut être déterminée par la donnée de $n$ formes de PFafF complexes $\omega^{s}=\alpha^{s}+i \alpha^{s^{\prime}}(s=1, \ldots, n)$ telles que les $2 n$ formes réelles $\alpha^{s}$, $\alpha^{s^{\prime}}$ soient linéairement indépendantes, les champs $C_{1}, C_{2}, C_{3}, C_{4}$ étant définis respectivement par les équations:

$$
\theta^{s^{\prime}}=\frac{\alpha^{s}-\alpha^{s^{\prime}}}{\sqrt{2}}=0, \quad \theta^{s}=\frac{\alpha^{s}+\alpha^{s^{\prime}}}{\sqrt{2}}=0, \quad \alpha^{s^{\prime}}=0, \quad \alpha^{s}=0 \quad(s=1, \ldots, n) .
$$

La même structure peut être déterminée par $2 n$ formes de PfafF complexes $\tilde{\omega}^{t}=\Sigma u_{s}^{t} \omega^{s}$, où les $u_{s}^{t}$ sont des fonctions locales différentiables à valeurs réelles, déterminant en chaque point $x \in U$ une transformation de $\widehat{L}_{n}$.

A la structure $\mathfrak{I}$ qui est à la fois subordonnée à une structure presque complexe et à deux structures presque paracomplexes, on peut associer cano. niquement trois connexions affines, distinctes en général:

1) la connexion $(A)$ que nous appellerons connexion associée au champ d'automorphismes $\mathfrak{J}_{x}$ (c'est-à-dire à la structure presque complexe), définie par les formes réelles $\omega_{t}^{s}$ (linénires en $\omega^{l}$ et $\overline{\omega^{l}}$ ) telles que:

$$
\begin{aligned}
& d \omega^{s}=\Sigma \omega^{t} \wedge \omega_{t}^{s}+\Sigma A_{l l}^{s} \omega^{t} \wedge \omega^{l}+\Sigma B_{t l}^{s} \bar{\omega}^{t} \wedge \bar{\omega}^{l}, \quad(s, t, l=1, \ldots, n) \\
& A_{t l}^{s}+A_{l t}^{s}=0, \quad B_{t l}^{s}+B_{l t}^{s}=0
\end{aligned}
$$

où les $A_{t l}^{\mathrm{s}}$ et $B_{t l}^{s}$ sont complexes.

2) la connexion $(B)$ associée au champ d'automorphismes $\mathfrak{H}_{x}$, définie par les formes réelles $\alpha_{t}^{s}$ (linéaires en $\alpha^{l}$ et $\alpha^{\eta}$ ) telles que:

$$
\begin{aligned}
& d \alpha^{s}=\Sigma \alpha^{t} \wedge \alpha_{t}^{s}+\Sigma a_{t l}^{s} \alpha^{t} \wedge \alpha^{l}+\Sigma a_{t \eta^{\prime}}^{s} \alpha^{t \prime} \wedge \alpha^{l^{\prime}}, \\
& d \alpha^{s^{\prime}}=\Sigma \alpha^{t^{\prime}} \wedge \alpha_{t^{\prime}}^{s^{\prime}}+\Sigma a_{t^{\prime} l^{\prime} \alpha^{t^{\prime}}}^{s^{\prime}} \wedge \alpha^{l^{\prime}}+\Sigma a_{t l}^{s^{\prime} \alpha^{t}} \wedge \alpha^{l}, \quad(s, t=1, \ldots, n) \\
& \alpha_{t}^{s}=\alpha_{t^{\prime}}^{s^{\prime}}, \quad a_{\beta \gamma}^{\delta}+a_{\gamma \beta}^{\delta}=0 \text {; } \\
& (\delta, \beta, \gamma=1, \ldots, 2 n)
\end{aligned}
$$

$\left.{ }^{9}\right)$ Ceci colrige ce qui a été écrit dans [39]. 
3) la connexion $(C)$ associée au champ d'automorphismes $\mathfrak{F}_{x}$, définie par les formes réelles $\theta_{i}^{s}$ (linéaires en $\theta^{s}$ et $\theta^{s^{\prime}}$ ) telles que:

$$
\begin{array}{ll}
d \theta^{s}=\Sigma \theta^{t} \wedge \theta_{t}^{s}+\Sigma b_{l}^{s} \theta^{t} \wedge \theta^{l}+\Sigma b_{t \eta^{\prime}}^{s} \theta^{t^{\prime}} \wedge \theta^{l^{\prime}}, & (s, t=1, \ldots, n) \\
d \theta^{s^{\prime}}=\Sigma \theta^{t^{\prime}} \wedge \theta_{t^{\prime}}^{s^{\prime}}+\Sigma b_{t^{\prime} l^{\prime}}^{g^{\prime}} \theta^{t^{\prime}} \wedge \theta^{l^{\prime}}+\Sigma b_{t l^{\prime}}^{s^{\prime}} \wedge \theta^{l}, & (\delta, \beta, \gamma=1, \ldots, 2 n) \\
\theta_{t}^{s}=\theta_{t^{\prime}}^{s^{\prime}}, \quad b_{\beta \gamma}^{\delta}+b_{\gamma \beta}^{\delta}=0 . &
\end{array}
$$

Les $a_{\beta \gamma}^{\delta}$ et $b_{\beta \gamma}^{\delta}$ sont réels.

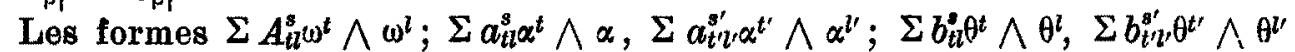
definissent respectivement la première torsion des connexions $(A),(B)$ et $(C)$.

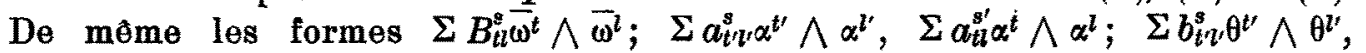
$\Sigma b_{l l}^{s^{\prime}} \mathrm{e}^{t} \wedge \theta^{l}$ définissent respectivement la deuxième torsion de ces connexions. On pourrait également pour chacune de ces connexions définir le tenseur de courbure et déduire les relations généralisant les identités de Bianchr. Comme pour les structures presque parahermitiennes (\$ 22), on peut démontrer que si l'un au moins des deux champs $C_{3}$ uu $C_{4}$ (resp. $C_{1}$ ou $C_{2}$ ) est complètement intégrable, la connexion induite par $(B)$ (resp. $(C)$ ) sur ses variétés intégrales est à courbure nulle.

On peut ecrire les équations $(30 B)$ par exemple, sous la forme:

$\left(30 B^{\prime}\right)$

$$
\begin{aligned}
d \theta^{\varepsilon}=\Sigma \theta^{t} \wedge \alpha_{t}^{8} & +\frac{1}{2 \sqrt{2}} \Sigma\left(a_{t l}^{s}+a_{t l}^{s^{\prime}}+a_{t^{\prime} l^{\prime}}^{s}+a_{t^{\prime} l^{\prime}}^{s^{\prime}}\right)\left(\theta^{i} \wedge \theta^{z}+\theta^{\prime \prime} \wedge \theta^{\prime}\right)+ \\
& +\frac{1}{2 \sqrt{\overline{2}}} \Sigma\left(a_{t l}^{s}+a_{t l}^{s^{\prime}}-a_{t^{\prime} l^{\prime}}^{s}-a_{t^{\prime} l^{\prime}}^{s^{\prime}}\right)\left(\theta^{t} \wedge \theta^{\prime \prime}+\theta^{\prime \prime} \wedge \theta^{\prime}\right) .
\end{aligned}
$$

$$
\begin{aligned}
& d \theta^{\theta^{\prime}}=\Sigma \theta^{t^{\prime}} \wedge \alpha_{t}^{s}+\frac{1}{2 \sqrt{2}} \Sigma\left(a_{t l}^{s}-a_{t l}^{s^{\prime}}+a_{t^{\prime} l^{\prime}}^{s}-a_{t^{\prime \prime}}^{s^{\prime}}\right)\left(\theta^{t} \wedge \theta^{l}+\theta^{t^{\prime}} \wedge \theta^{\prime \prime}\right)+ \\
& +\frac{1}{2 \sqrt{2}} \Sigma\left(a_{t l}^{s}-a_{l l}^{s t}-a_{t^{\prime}{ }^{\prime}}^{s}+a_{t^{\prime}}^{s^{\prime}}\right)\left(\theta^{t} \wedge \theta^{y}+\theta^{t^{\prime}} \wedge \theta^{\prime}\right)
\end{aligned}
$$

ou sous la forme:

$$
\begin{aligned}
d \omega^{s}=\Sigma \omega^{t} \wedge \alpha_{t}^{s} & +\frac{1}{4} \Sigma\left(a_{t l}^{s}+i a_{t l}^{s^{\prime}}-a_{t^{\prime} l^{\prime}}^{s}-i a_{t^{\prime}}^{\mathrm{g}^{\prime}}\right)\left(\omega^{t} \wedge \omega^{l}+\bar{\omega}^{t} \wedge \bar{\omega}^{l}\right)+ \\
& +\frac{1}{4} \Sigma\left(a_{t l}^{s}+i a_{t l}^{s^{\prime}}+a_{t^{\prime} l^{\prime}}^{s}+i a_{t^{\prime} l}^{s^{\prime}}\right)\left(\omega^{t} \wedge \bar{\omega}^{l}+\bar{\omega}^{t} \wedge \omega^{l}\right)
\end{aligned}
$$

d'où :

$$
\begin{aligned}
& \theta_{t}^{s}=\alpha_{t}^{s}+\frac{1}{\sqrt{2}} \Sigma\left(a_{t l}^{s}+a_{l t}^{s^{f}}-a_{v^{\prime}}^{s}-a_{t v}^{s^{\prime}}\right) \theta^{y}+ \\
& +\frac{1}{\sqrt{\overline{2}}} \Sigma\left(a_{t l}^{s}-a_{t l}^{s^{\prime}}-a_{t^{\prime} v^{\prime}}^{s}+a_{t^{\prime} v}^{s^{\prime}}\right) \theta^{l} ; \\
& \omega_{t}^{s}=\alpha_{t}^{s}+\frac{1}{2} \Sigma\left(a_{t l}^{s}+i a_{t l}^{s^{\prime}}+a_{t^{\prime} \nu}^{s}+i a_{t^{\prime}{ }^{\prime}}^{s^{\prime}}\right) \bar{\omega}^{l}+ \\
& +\frac{1}{2} \Sigma\left(a_{t l}^{s}-i a_{t l}^{a^{\prime}}+a_{t v}^{s}-i a_{t v}^{s \prime}\right) \omega^{l}
\end{aligned}
$$


Denx des connexions corncident si $\alpha_{t}^{s}=\omega_{t}^{s}$ ou $\alpha_{t}^{s}=\theta_{t}^{s}$ ou $\theta_{t}^{s}=\omega_{t}^{s}$ $(s, t=1, \ldots, n)$; d'où :

Théontime 30.1 - Pour que deux des trois connexion coìnoident, il faut et il suffit que la deuxième torsion de la troisième connexion soit nulle.

Par conséquent si la structure dérive d'une structure complexe, les connexions associées aux automorphismes $\mathfrak{F}_{x}$ et $\mathfrak{H}_{x}$ colncident (on a alors: $\left.\alpha_{i}^{s}=\theta_{t}^{s}\right)$.

En particulier pour que les trois connexions coincident, il faut et il suffit que la deuxième torsion de deux des connexions soit nulle; cette condition réalisée, en raison des formules $(30 \mathrm{~B})$ par exemple, la première torsion de la connexion unique est nulle. Inversement si l'une des connexions est a torsion nulle, les trois connexions coilncident. Les quatre champs $C_{1}, C_{2}, C_{3}$, $\mathrm{O}_{4}$ sont alors complètement intégrables. Il résulte d'une propriété démontrée précédemment que la connexion induite sur les variétés intégrales des quatre champs est à courbure nulle; la torsion étant nulle, la structure induite sur ces variétés est intégrable: D'où :

Thtorime 30.2. - Pour que les trois connexions coincident, il faut et il suffit que les quatre champs $C_{1}, \dot{C}_{2}, C_{3}, C_{4}$ soient complètement intégrables. La structure induite sur leurs variétés intégrales est alors intégrable.

Tout champ $C^{(k)}$ de $n$-éléments $X_{n}$ défini localement par les équations:

$$
\alpha^{s}+\mu \alpha^{s^{\prime}}=0, \quad(s=1, \ldots, n),
$$

où $\mu$ est une constante, est de même complètement intégrable. Ces champs de $n$-éléments définissent sur $V_{2 n}$ une famille à un paramètre de feuilletages.

Si les données sont analytiques, la torsion étant nulle, la structure sur $V_{2 n}$ dérive d'une structure complexe.

Pour que la structure $\mathfrak{I}$ soit intégrable, il faut et il suffit que la cour. bure et la torsion de l'une des connexions soient nulles (en raison d'une remarque de \& 6j; les trois connexions coincident alors. Il existe dans le voisinage de tout point de $V_{2 n}$ un système de coordonnées locales $x^{1}, \ldots, x^{n}$, $y^{1}, \ldots, y^{n}$ tel que les champs $C_{1}, C_{2}, C_{3}, C_{1}$ soient definis respectivement par les equations:

$$
d\left(x^{s}-y^{s}\right)=0, \quad d\left(x^{s}+y^{s}\right)=0, \quad d y^{s}=0, \quad d x^{s}=0, \quad(s=1, \ldots, n) .
$$

Suivant le théorème 6.2 le pseudogroupe des automorphismes locaux de la structure est localement équivalent au groupe affine de $R^{2 n}$ dont le groupe $\widehat{L_{n}}$ est le plus grand sous-groupe homogène. On peut d'ailleurs démontrer directement que les changements de coordonnées locales

$$
\begin{aligned}
& X^{s}=f^{s}\left(x^{1}, \ldots, x^{n}\right), \\
& Y^{s}=g^{s}\left(x^{1}, \ldots, y^{n}\right),
\end{aligned} \quad(s=1, \ldots, n)
$$


tels que les champs $C_{1}, C_{2}, C_{3}, C_{4}$ puissent être définis par: $d\left(X^{s}-Y^{s}\right)=0$, $d\left(X^{s}+Y^{s}\right)=0, d Y^{s}=0, d X^{s}=0$ sont des changements de coordonnées linéaires.

Dans la suite de ce paragraphe, nous supposerons $n$ pair $(n=2 p)$. Les indices latins varieront de $1 \stackrel{a}{a} p$, les indices grees de 1 à $2 p$, avec $s^{\prime}=s+p$, La structure $\mathfrak{I}$ est alors nne structure presque quaternionienne de deuxieme espèce; dans un voisinage $U$ elle peut être déterminée par les $2 p$ formès de PFaff complexes lineairement independantes $\pi^{s}=\omega^{s}+i \omega^{s^{\prime}}, \pi^{s^{\prime}}=\omega^{8}-i \omega^{s^{\prime}}$ $(s=1, \ldots, n)$ ou par $2 p$ autres formes $\tilde{\pi}^{\beta}=\Sigma a_{\alpha}^{\beta} \pi^{\alpha}$, les fonctions complexes $a_{\alpha}^{\beta}$ définissant en chaque point une transformation du groupe linéaire quaternionionien de deuxième espèce $\check{L}_{p}^{\prime \prime}\left(a_{t}^{s}=\bar{a}_{t^{\prime}}^{s^{\prime}}, a_{t^{\prime}}^{s}=\bar{a}_{t}^{s^{\prime}}\right)$.

Les equations $(30 \mathrm{~A})$ définissant la connexion affine associée aux automorphismes $\mathfrak{d}_{x}$ peuvent s'écrire :

$$
\begin{aligned}
& d \pi^{s}=\Sigma \pi^{t} \wedge \pi_{t}^{s}+\Sigma \pi^{t^{\prime}} \wedge \pi_{t^{\prime}}^{s}+\Sigma H_{\alpha \beta}^{s} \pi^{\alpha} \wedge \pi^{\beta}+\Sigma K_{\alpha \beta}^{s} \bar{\pi}^{\alpha} \wedge \overline{\pi^{\beta}},(s, t=1, \ldots, p) \\
& \left(30 A^{\prime}\right) \quad d \pi^{s^{\prime}}=\Sigma \pi^{t} \wedge \pi_{t}^{s^{\prime}}+\Sigma \pi^{t^{\prime}} \wedge \pi_{t^{\prime}}^{s^{\prime}}+\Sigma H_{\alpha \beta}^{s^{\prime}} \pi^{\alpha} \wedge \pi^{\beta}+\Sigma K_{\alpha \beta}^{s^{\prime}} \bar{\pi}^{\alpha} \wedge \overline{\pi^{\beta}} \\
& \pi_{t}^{s}=\bar{\pi}_{t^{\prime}}^{s^{\prime}}, \quad \pi_{t}^{s^{\prime}}=\bar{\pi}_{t^{\prime}}^{s} .
\end{aligned}
$$

$\left(0 \mathrm{n} \mathrm{a} \mathrm{:} 2 \pi_{t}^{s}=\omega_{t}^{s}+i \omega_{t}^{s t}+\omega_{t^{\prime}}^{s}-i \omega_{t^{\prime}}^{s}, 2 \pi_{t^{\prime}}^{s}=\omega_{t}^{s}+i \omega_{t}^{s \prime}-\omega_{\nu}^{s \prime}+i \omega_{t^{\prime}}^{s}\right)$.

Une structure presque quaternionienne de deuxième espèce intégrable sera dite quaternionienne de deuxième espèce. D'après ce qui a été démontré précédemment, on obtient seulement des variétés localement affines.

Remarquons $q u^{\prime}$ une structure presque quaternionienne peut être définie localement par $2 p$ formes de PFAFF complexes $\pi^{\alpha}$ ou par $2 p$ autres formes $\tilde{\pi}^{\beta}=\Sigma a_{a}^{\beta} \pi^{\alpha}\left(\right.$ arec $\left.a_{t}^{s}=\bar{a}_{t^{\prime}}^{s^{\prime}}, a_{t}^{s^{\prime}}=-\bar{a}_{t}^{s}\right)$. On peut associer canoniquement ̀े une telle structure une connexion affine definie par des equations analogues à $(30 \mathrm{~A})$ mais où les formes $\pi_{\beta}^{\alpha}$ vérifient les relations :

$$
\pi_{s}^{t}=\tau_{s^{\prime}}^{t^{\prime}}, \quad \pi_{s}^{\prime \prime}=-\bar{\pi}_{s^{\prime}}^{t} \quad(s, t=1, \ldots, p) .
$$

C. Ehresmann a démontré [24] que tonte variété quaternionienne est locale. ment affine.

\section{Courbure et torsion conformes.}

Supposons définie une structure $\mathfrak{I}$ sur une variété $V_{2 n}$ ( $n$ étant quelconque); considérons la famille $\mathfrak{\Xi}_{\mu_{2} \mu_{2} \mu_{3}}$ de structures de même espèce. Si l'on fixe en chaque point l'automorphisme $\mathfrak{J}_{x}$ (c'est-à-dire la structure presque complexe), on obtient une famille $\mathfrak{F}_{h_{1}}$ de structures (dépendant d'une fonction arbitraire $\lambda_{1}$ ); chacune de ces structures peut être définie localement par les $n$ formes complexes

$$
\pi_{\left(\lambda_{1}\right)}^{s}=\lambda_{1} \omega^{s}=\lambda_{1}\left(\alpha^{s}+i \alpha^{s^{\prime}}\right) \quad\left(s=1, \ldots, n ; s^{\prime}=s+n\right)
$$


la structure $\mathfrak{I}$ étant déterminée par les formes $\alpha^{s}$, $\alpha^{s^{\prime}}$ on $\theta^{s}, \theta^{s^{\prime}}$ définies par (30.1); on peut supposer la fonction $\lambda_{1}$ de module $1\left(\lambda_{1}=\cos \rho_{1}+i \sin \rho_{1}\right.$ où $\rho_{1}$ est réel). Les champs $\left(C_{1}\right)_{\lambda_{1}},\left(C_{2}\right)_{\lambda_{1}},\left(C_{3}\right)_{\lambda_{1}},\left(C_{4}\right)_{\lambda_{1}}$ associés a la structure ${ }_{\lambda_{1}}$ sont définis respectivement par les équations :

$$
\begin{aligned}
\left(\alpha^{3}-\alpha^{s^{\prime}}\right) \cos \rho_{1}-\left(\alpha^{3}+\alpha^{s^{\prime}}\right) \sin \rho_{1}=0, & \left(\alpha^{s}+\alpha^{s^{\prime}}\right) \cos \rho_{1}+\left(\alpha^{s}-\alpha^{s^{\prime}}\right) \sin \rho_{1}=0, \\
(31.2) \alpha^{s} \sin \rho_{1}+\alpha^{s^{\prime}} \cos \rho_{1}=0, & \alpha^{s} \cos \rho_{1}-\alpha^{s^{\prime}} \sin \rho_{1}=0, \\
& (s=1, \ldots, n) .
\end{aligned}
$$

Les automorphismes $\left(\mathscr{F}_{x}\right) \lambda_{\lambda_{1}}$ et $\left(\mathfrak{K}_{x}\right)_{\lambda_{1}}$ peuvent être représentés respectivement par:

$$
\mathfrak{F}_{x} \cos 2 \rho_{1}-\mathfrak{K}_{x} \sin 2 \rho_{1}, \quad \mathfrak{F}_{x} \sin 2 \rho_{1}+\mathfrak{H}_{x} \cos 2 \rho_{1} .
$$

De même que pour les structures presque hermitiennes et presque parahermitiennes (\$25), on peut definir une courbure et une torsion conformes correspondant à la connexion $(A)$ (associée aux automorphismes $\mathfrak{J}_{x}$ ). En dési. gnant par $\Omega^{s}, \Gamma^{s}, \Omega_{t}^{s}$ les formes definissant respectivement la premiere torsion, la deuxième torsion, la conrbure de la connexion $(A)$, on definit la courbure conforme par:

$$
\Pi_{s}^{t}=\Omega^{t}-\delta_{s}^{t} \frac{\Sigma \Omega_{l}^{l}}{n}
$$

la première torsion conforme par :

$$
\Pi^{s}=\Omega^{s}-\frac{\varphi_{1} \wedge \omega^{s}}{n-1}
$$

où $\varphi_{1}=2 \Sigma A_{t s}^{8} \omega^{t}$ est invariante par tout changement de corepère appartenant a $\widehat{L}_{11}$; la deuxième torsion conforme est par définition identique à la deuxième torsion. La forme $\Phi_{1}=\varphi_{1}-\bar{\varphi}_{1}$ transformée en $\Phi_{1}^{\prime}=\Phi_{1}+2(n-1) \frac{d \lambda}{\lambda}$ par la transformation $\pi^{s}=\lambda_{1} \omega^{s}$ sera appelée forme de torsion associée à la connexion (A) (of. structures presque symplectiques et structures subordonnées). Le même raisonnement que pour les structures presque hermitiennes et presque parahermitiennes (\$ 25) conduit aux theorèmes suivants :

TнEовіме 31.1. - Étant donnée une structure $\leqq$, pour qu'il existe une fonction différentiable $\lambda_{1}$ telle que la structure $\mathfrak{S}_{\lambda_{1}}$ soit intégrable il faut et pour $n>2$, il suffit que la courbure et la torsion conformes soient nulles.

Si $n>2, \Pi^{s}=0$ et $\Pi_{t}^{s}=0$ entrainent $d \Phi_{1}=0$. Si $n=2$, on a $\Pi^{s}=0$ identiquement mais il faut imposer, outre la condition $\Pi_{t}^{s}=0$, la condition $d \Phi_{1}=0$ pour que la structure $\Xi_{\lambda_{1}}$ soit intégrable.

Les conditions du théorème 31.1 étant réaliseós la fonction $\lambda_{1}$ est définie à un facteur constant près. On démontre de manière analogue le théorème suivant:

THEOREM 31.2. - Pour $q u$ 'il existe une fonction $\lambda_{4}$ telle que les champs $\left(C_{4}\right)_{\lambda_{1}},\left(C_{2}\right)_{\lambda_{1}},\left(C_{3}\right)_{\lambda_{2}},\left(C_{4}\right)_{\lambda_{1}}$ soient complètement intégrables, il faut et il suffit que la torsion conforme et la différentielle de la forme de torsion soient nulles. 
Contrairement aux structures presque symplectiques (et structures subordonnées), la condition $\Pi^{s}=0$ n'entraine pas nécessairement $d \Phi_{1}=0$.

En fixant en chaque point l'automorphisme $\mathfrak{J}_{x}$ (resp. $\mathfrak{A}_{x}$ ), on obtient des structures $\mathfrak{F}_{\lambda_{2}}$ (resp. $\mathfrak{F}_{\lambda_{3}}$ ) dependant d'une fonction arbitraire $\lambda_{2}$ (resp. $\lambda_{3}$ ). Si l'on fixe $\mathscr{F}_{x}$ (e'est-à-dire les $n$-éléments $X_{n}$ et $X_{n}{ }^{\prime}$ ), chaque structure $\mathfrak{I}_{\lambda_{2}}$ peut être déterminée localement par les $\boldsymbol{n}$ formes paracomplexes

$$
\pi_{\left(\lambda_{2}\right)}^{s}=\theta_{\left(\lambda_{2}\right)}^{s}+j \theta_{\left(\lambda_{2}\right)}^{s}=\lambda_{2}\left(\theta^{s}+j \theta^{s^{\prime}}\right) \quad(s=1, \ldots, n)
$$

où $\lambda_{2}$ est un nombre paracomplexe que l'on peut supposer de norme 1 $\left(\lambda_{2}=\operatorname{ch} \rho_{2}+j \operatorname{sh} \rho_{2}, \rho_{2}\right.$ étant réel); on $a$ :

$$
\left(\mathfrak{J}_{x}\right)_{\lambda_{2}}=\mathscr{J}_{x} \operatorname{ch} 2 \rho_{z}-\mathfrak{H}_{x} \operatorname{sh} 2 \rho_{2}, \quad(\mathscr{H})_{\lambda_{2}}=\mathfrak{J}_{x} \operatorname{sh} 2 \rho_{2}+\mathfrak{R}_{x} \operatorname{ch} 2 \rho_{2} .
$$

Si l'on fixe $\mathfrak{R}_{x}$ (e'est-à-dire $Y_{n}$ et $Y_{n}{ }^{\prime}$ ), chacune des structures $\dddot{I}_{\lambda_{s}}$ peut être déterminée localement par les $\boldsymbol{n}$ formes paracomplexes

$$
\pi_{\left(\lambda_{3}\right)}^{s}=\alpha_{\left(\lambda_{3}\right)}^{s}+j \alpha_{\left(\lambda_{3}\right)}^{s t}=\lambda_{3}\left(\alpha^{s}+j \alpha^{s^{\prime}}\right) \quad(s=1, \ldots, n)
$$

où $\lambda_{3}=\operatorname{ch} \rho_{3}+j \operatorname{sh} \rho_{3} ;$ d'où les automorphismes

$$
\left(\mathfrak{J}_{x}\right)_{\lambda_{3}}=\mathfrak{J}_{x} \operatorname{ch} 2 \rho_{3}-\mathfrak{F}_{x} \operatorname{sh} 2 \rho_{3}, \quad\left(\mathfrak{F}_{x}\right)_{\lambda_{s}}=\mathfrak{I}_{x} \operatorname{sh} 2 \rho_{3}+\mathfrak{F}_{x} \operatorname{ch} 2 \rho_{3} .
$$

On peut définir pour chacune des connexions $(B)$ et $(C)$ une courbure et une torsion conformes, ainsi qu' une forme de torsion. Les théorèmes 31.1 et 31.2 s' appliquent aux structures $\mathfrak{F}_{\lambda_{2}}$ et $\mathfrak{s}_{\lambda_{3}}$.

32. Structures à groupe structural isomorphe au groupe orthogonal $O_{n}$.

Lẹ même raisonnement que pour les structures presque hermitiennes et presque parahermitiennes montre que les seules structures $\mathfrak{I}$ isotropes sont intégrables; par contre nous allons montrer qu'on obtient des structures isotropes non intégrables en considérant des structures $\mathfrak{I}^{\prime}$ subordonnées aux structures $\mathfrak{\Xi}$, de groupe structural isomorphe à $O_{n}$.

Si l'on se donne sur une variété $V_{2 n}$ une structure $\$$ [déterminée par exemple par un triplet $\left(C_{1}, C_{2}, C_{3}\right)$ de champs de $n$-éléments $\left.X_{n}, X_{n}{ }^{\prime}, Y_{n}\right]$ et une forme différentielle extérieure $\Omega$ (de degré 2 , de rang $2 n$ ) telle que les $n$-éléments $X_{n}, X_{n}^{\prime}, Y_{n}$ soient intégraux de $\Omega$, on definit sur $V_{2 n}$ une structure $\boldsymbol{S}^{\prime}$ dont le groupe structural $G$ est isomorphe à $O_{n}$ : cette structure est subordonnée à une structure presque hermitienne et à deux structures presque parahermitiennes. Localement une structure $\mathfrak{\Xi}^{\prime}$ peut être déterminée par la donnée de $n$ formes de PFAFF complexes linéairement indépendantes $\omega^{s}=\alpha^{s}+i \alpha^{s}(s=1, \ldots, n)$ telles que la forme $\Omega$ s' écrive: $\Omega=i \Sigma \omega^{s} \wedge \bar{\omega}^{s}=$ $=2 \alpha^{s} \wedge \alpha^{s^{\prime}}$, le champs $C_{1}, C_{2}, C_{2}, C_{4}$ etant définis par les équations (30.1). A cette structure sont associees une métrique hermitienne $\psi=\Sigma \omega^{s} \bar{\omega}^{s}$ et deux métriques parahermitiennes $\psi_{1}^{\prime}=\Sigma\left(\alpha^{s}+j \alpha^{\prime}\right)\left(\alpha^{s}-j \alpha^{s^{\prime}}\right), \psi_{2}^{\prime}=\Sigma\left(\theta^{s}+j \theta^{s^{\prime}}\right)\left(\theta^{s}-j \theta^{s^{\prime}}\right)$. 
d' où la métrique riemannienne définie positive $F=\Sigma\left[\left(\alpha^{s}\right)^{2}+\left(\alpha^{s}\right)^{2}\right]=$ $=\Sigma\left[\left(\theta^{s}\right)^{2}+\left(\theta^{s^{\prime}}\right)^{2}\right]$ et les métriques riemanniennes indéfinies

$$
F_{1}^{\prime}=\Sigma\left[\left(\alpha^{s}\right)^{2}-\left(\alpha^{z^{\prime}}\right)^{2}\right]=2 \Sigma \theta^{8} \theta^{s^{\prime}}, \quad F_{2}^{\prime}=\Sigma\left[\left(\theta^{s}\right)^{2}-\left(\theta^{s^{\prime}}\right)^{2}\right]=2 \Sigma \alpha^{8} \alpha^{z^{\prime}} .
$$

On peut associer canoniquement à la structure $\mathfrak{I}^{\prime}$ six connexions affines en général distinctes: une connexion hermitienne, deux connexions parahermitiennes et trois connexions riemanniennes.

Si les champ $C_{1}$ et $C_{2}$ (resp. $C_{3}$ et $C_{4}$ ) sont complètement intégrables, la métrique riemannienne $F$ est localement réductible [36] c' est-à-dire il existe dans le voisinage de tout point de $V_{2 n}$ un système de coordonnées locales $x^{1}, \ldots, x^{n}, y^{1}, \ldots, y^{n}$ tel que $F=f_{1}+f_{2}$ ou $f_{1}=\Sigma g_{s l}(x) d x^{s} d y^{l}$, $f_{2}=\Sigma h_{s i}(y) d y^{s} d y^{l}$.

Si les quatre champs $C_{1}, C_{2}, C_{3}, C_{4}$ sont complètement intégrables, la connexion hermitienne et les connexions parahermitiennes coincident; la structure induite sur les variétés intgérales des quatre champs est une structure d' espace localement euclidien.

En cherchant les structures $\Psi^{\prime}$ isotropes, on est conduit [par le même raisonnement que pour les structures presque hermitiennes (\$ 23)] aux structures $\mathfrak{I}^{\prime}$ intégrables (pour $n$ quelconque) et de plus, pour $n=3$, à des structures déterminées localement par des formes réelles $\alpha^{s}, \alpha^{s^{\prime}}$ qui vérifient les oquations :

$$
\begin{aligned}
& d \alpha^{1}=\alpha^{2} \wedge \alpha_{2}^{1}+\alpha^{3} \wedge \alpha_{3}^{1}+a \alpha^{2} \wedge \alpha^{3}+b \alpha^{5} \wedge \alpha^{6}, \\
& d \alpha^{2}=\alpha^{1} \wedge \alpha_{1}^{2}+\alpha^{3} \wedge \alpha_{3}^{2}+a \alpha^{3} \wedge \alpha^{1}+b \alpha^{6} \wedge \alpha^{4}, \\
& d \alpha^{3}=\alpha^{1} \wedge \alpha_{1}^{3}+\alpha^{2} \wedge \alpha_{2}^{3}+a \alpha^{1} \wedge \alpha^{2}+b \alpha^{4} \wedge \alpha^{5}, \\
& d \alpha^{4}=\alpha^{5} \wedge \alpha_{2}^{1}+\alpha^{6} \wedge \alpha_{3}^{4}+\alpha^{\prime} \alpha^{5} \wedge \alpha^{6}+b^{\prime} \alpha^{2} \wedge \alpha^{3}, \\
& d \alpha^{5}=\alpha^{4} \wedge \alpha_{1}^{2}+\alpha^{6} \wedge \alpha_{3}^{2}+a^{\prime} \alpha^{8} \wedge \alpha^{4}+b^{\prime} \alpha^{3} \wedge \alpha^{1}, \\
& d \alpha^{6}=\alpha^{4} \wedge \alpha_{1}^{3}+\alpha^{5} \wedge \alpha_{2}^{3}+a^{\prime} \alpha^{4} \wedge \alpha^{5}+b^{\prime} \alpha^{1} \wedge \alpha^{2}, \\
& d \alpha_{1}^{2}=\alpha_{1}^{3} \wedge \alpha_{3}^{1}+\alpha^{\prime} b^{\prime} \alpha^{1} \wedge \alpha^{2}+a b \alpha^{4} \wedge \alpha^{5}-b b^{\prime}\left(\alpha^{1} \wedge \alpha^{5}+\alpha^{2} \wedge \alpha^{4}\right), \\
& d \alpha_{2}^{3}=\alpha_{2}^{1} \wedge \alpha_{1}^{3}+a^{\prime} b^{\prime} \alpha^{2} \wedge \alpha^{3}+a b \alpha^{5} \wedge \alpha^{6}-b b^{\prime}\left(\alpha^{2} \wedge \alpha^{5}+\alpha^{3} \wedge \alpha^{5}\right), \\
& d \alpha_{3}^{1}=\alpha_{3}^{2} \wedge \alpha_{2}^{1}+\alpha^{\prime} b^{\prime} \alpha^{3} \wedge \alpha^{1}+a b \alpha^{6} \wedge \alpha^{4}-b b^{\prime}\left(\alpha^{3} \wedge \alpha^{4}+\alpha^{1} \wedge \alpha^{6}\right), \\
& \alpha_{s}^{t}+\alpha_{t}^{3}=0 \quad(s, t=1,2,3)
\end{aligned}
$$

$a, b, a^{\prime}, b^{\prime}$ etant des constantes.

La structure $\mathfrak{S}^{\prime}$, au lieu d'être déterminée par les champs $C_{1}, C_{2}, C_{3}$ de n-éléments $X_{n}, X_{n}^{\prime}, Y_{n}$ peut être déterminée par tout triplet $\left(C^{\prime\left(\mu_{1}\right)}, C^{\left(\mu_{2}\right)}, C^{\left(\mu_{3}\right)}\right)$ de champs de $n$-éléments où $\mu_{1}, \mu_{2}, \mu_{3}$ sont des constantes. Cherchons s' il existe des champs $C^{(\mu)}$ définis par les équations $\alpha^{s}+\mu \alpha^{\prime}=0(s=1, \ldots, n)$ qui sont complètement intégrables; pour cela il faut et il suffit que le nombre $\mu$ vérifie la relation:

$$
\mu^{3} b^{\prime}+\mu^{2} a+\mu a^{\prime}+b=0 .
$$


Si l'équation (32.2) a 3 racines réelles distinctes, les équations (32.1) peuvent se ramener à :

$$
\begin{array}{ll}
d \alpha^{1}=\alpha^{2} \wedge \alpha^{3}, & d \alpha^{4}=\alpha^{5} \wedge \alpha^{6} \\
d \alpha^{2}=\alpha^{3} \wedge \alpha^{1}, & d \alpha^{5}=\alpha^{6} \wedge \alpha^{4} \\
d \alpha^{3}=\alpha^{1} \wedge \alpha^{2}, & d \alpha^{6}=\alpha^{4} \wedge \alpha^{5}
\end{array}
$$

en choisissant en chaque point le corepère de façon que les $\alpha_{l}^{s}$ soient nuls; d'où une structure $\mathfrak{g}^{\prime}$ sur la variété $S_{3} \times S_{3}$.

Si l'équation (32.2) a une racine simple et une racine double, les équations (32.1) peuvent se ramener à :

$$
\begin{array}{ll}
d \alpha^{1}=\alpha^{2} \wedge \alpha^{3}, & d \alpha^{4}=0 \\
d \alpha^{2}=\alpha^{3} \wedge \alpha^{1}, & d \alpha^{5}=0 \\
d \alpha^{3}=\alpha^{1} \wedge \alpha^{2}, & d \alpha^{6}=0
\end{array}
$$

d'où une structure $\mathfrak{I}^{\prime}$ sur la variété $S_{3} \times R^{3}$ (l'espace numérique $R^{3}$ étant muni de sa structure d'espace euclidien).

Si l'équation (32.2) a une racine réelle et deux racines imaginaires conjuguées, les équations peuvent se ramener à:

$$
\begin{aligned}
& d \omega^{1}=\omega^{2} \wedge \omega^{3} \\
& d \omega^{2}=\omega^{3} \wedge \omega^{1} \\
& d \omega^{3}=\omega^{1} \wedge \omega^{2}
\end{aligned}
$$

les $\omega^{s}$, étant complexes; d'où une structure $\mathfrak{\Xi}^{\prime}$ sur la variété du groupe des rotations complexes à trois paramètres.

Si l'équation (32.2) a une racine triple, on se ramène aux équations:

$$
\begin{array}{ll}
d \alpha^{1}=0, & d \alpha^{4}=\alpha^{2} \wedge \alpha^{3} \\
d \alpha^{2}=0, & d \alpha^{5}=\alpha^{3} \wedge \alpha^{1} \\
d \alpha^{3}=0, & d \alpha^{6}=\alpha^{1} \wedge \alpha^{2}
\end{array}
$$

On obtient les equations de structure d'un groupe $g$ à 6 paramètres, extension de $R^{3}$ par $R^{3}$ [7].

En résumê on obtient le théorème suivant:

THÉonÈme 32.1. - Une structure $\mathfrak{I}^{\prime}$ isotrope est localement homogène; cette structure est intégrable ou bien localement équivalente à une structure de même espèce sur l'une des variétés suivantes: $S_{3} \times S_{3}, S_{3} \times R^{3}$, variété du groupe g extension de $R^{3}$ par $R^{3}$ (si la structure ne dérive pas d'une structure complexe), variété du groupe des rotations complexes à 3 paramètres (si la structure dérive d'une structure complexe). 
33. Structures à groupe structural isomorphe à $L_{p} \times L_{p} \times L_{p} \times L_{p}$, à $L_{p}^{\prime} \times L_{p}^{\prime}$ on à un de leur sons-groupes.

En raison de l'étude faite dans $\S 12$, sur une variété $V_{4 p}$ de dimension $4 p$, une structure infinitésimale régulière de groupe structural isomorphe a $L_{p} \times L_{p} \times L_{p} \times L_{p}$ peut être déterminée pạr la donnée d' un ensemble ordonne $\left(C_{1}, C_{2}, C_{3}, C_{4}\right)$ de quatre champs deux fois différentiables de p-éléments de contact deux à deux supplémentaires ou par la donnée de l'un des couples $(\mathfrak{J}, \mathfrak{F}),(\mathfrak{F}, \mathfrak{K}),(\mathfrak{A}, \mathfrak{d})$ de champs de $(2 p, 2 p)$-involutions $\mathfrak{J}_{x}$, $\mathfrak{J}_{x}, \mathfrak{K}_{x}$ de l'espace tangent $T_{x}$ à $V_{2 n}$, telles que: $\mathfrak{J}_{x} \mathfrak{J}_{x}=\mathfrak{F}_{x} \mathfrak{d}_{x}=\mathfrak{H}_{x}$.

Sur une variété $V_{4 p}$ une structure infinitésimale régulière de groupe structural isomorphe a $L_{p}^{\prime} \times L_{p}^{\prime}$ peut être déterminee par la donnée d'une structure presque complexe et de deux champs deux fois differentiables $C_{1}$ et $C_{2}$ d'éléments de contact complexes (of. $\S 10$ ) supplémentaires $X_{2 p}$ et $X_{2 p}^{\prime}$ ou par la donnée d'un champ de $(2 p, 2 p)$-involutions $\partial \mathcal{K}_{x}$ et d'un champ d'auto. morphismes $\mathfrak{I}_{x}$ tels que : $\left(\mathfrak{d}_{x}\right)^{2}=-1, \mathfrak{J}_{x} \mathfrak{H}_{x}=\mathfrak{H}_{x} \mathfrak{d}_{x}$.

Dans un voisinage $U$ ùne telle structure peut être déterminée par la donnée de $2 p$ formes de PFAFF complexes linéairement indépendantes telles que les champs $C_{1}$ et $C_{2}$ soient définis respectivement par :

$$
\omega^{\mathrm{g}^{\prime}}=0, \quad \omega^{s}=0 \quad\left(s=1, \ldots, p ; s^{\prime}=s+p\right) .
$$

La même structure peut être déterminée par les formes $\pi^{l}=\Sigma u_{s}^{l} \omega^{s}$, $\pi^{v^{\prime}}=\Sigma u_{s^{\prime}}^{l^{\prime}}\left(\mathrm{s}^{s^{\prime}}\right.$ où les $u_{s}^{l}$ ot $u_{s^{\prime}}^{l^{\prime}}$ sont des fonctions différentiables complexes. Les différentielles $d \omega^{x}$ peuvent s'écrire :

$$
\begin{aligned}
& d \omega^{s}=\Sigma \omega^{t} \wedge \omega_{t}^{s}+\Omega^{s}, \\
& d \omega^{s^{\prime}}=\Sigma \omega^{t^{\prime}} \wedge \omega_{t^{\prime}}^{s^{\prime}}+\Omega^{s^{\prime}},
\end{aligned} \quad\left(s=1, \ldots, p ; s^{\prime}=s+p\right)
$$

où les formes complexes $\omega_{t}^{s}$ (définies mod. $\omega^{s}$ ) et $\omega_{t^{\prime}}^{s^{\prime}}$ (définies mod. $\omega^{s^{s}}$ ) sont linéaires en $\omega^{k}, \omega^{k^{\prime}}, \bar{\omega}^{h}, \bar{\omega}^{k^{\prime}}$, les formes $\Omega^{s}$ et $\Omega^{s^{\prime}}$ qui définissent la torsion étant des formes différentielles extérieures quadratiques $\left(\Omega^{s}\right.$ en $\omega^{k^{\prime}}, \bar{\omega}^{k}, \bar{\omega}^{k^{\prime}}$, $\Omega^{s^{\prime}}$ en $\left.\omega^{k}, \bar{\omega}^{h}, \bar{\omega}^{k}\right)$. On ne peut associer canoniquement une connexion affine à cette structure (qui dérive de deux structures presque complexes correspondant aux champs d'automorphismes $\mathfrak{I}_{x}$ et $\mathfrak{J}_{x}=\mathfrak{J}_{x} \mathfrak{X}_{x}$ ).

Si de plus, on se donne dans l'espace tangent à $V_{t p}$ en chacun de ses points (muni de sa structure d'espace vectoriel complexe) une forme différentielle extérieure complexe de rang $2 p$ dont les sous-espaces $X_{2 p}$ et $X_{2 p}^{\prime}$ sont des éléments intégraux, on détermine sur $V_{4 p}$ une structure $\mathcal{S}$ dont le groupe structural $G$ est isomorphe a $L_{p}^{\prime}$; en effet localement la structure peut être déterminée par les formes $\omega^{s}$ et $\omega^{s^{\prime}}$ ou par les formes $\pi^{l}=\Sigma u_{s}^{l} \omega^{s}$ et $\pi^{\prime \prime}=\Sigma u_{g^{\prime}}^{v} \omega^{s^{\prime}}$ telles que $\Omega$ puisse s'écrire: $\Sigma \omega^{s} \wedge \omega^{s^{\prime}}=\Sigma \pi^{l} \wedge \pi^{\prime}$, et l'on a: $\Sigma u_{l}^{s} u_{k^{\prime}}^{s^{\prime}}=\delta_{l}^{k}$; le groupe $G$ est le groupe $\tilde{l}_{2 p}$ défini dans $\$ 14$. 
On peut associer canoniquement une connexion affine $a$ cette structure; on peut en effet déterminer de façon unique les formes $\omega_{t}^{s}$ et $\omega_{t^{\prime}}^{s^{\prime}}$ qui vérifient les équations :

$$
\begin{aligned}
& d \omega^{s}=\Sigma \omega^{t} \wedge \omega_{t}^{s}+\Omega^{s}, \\
& d \omega^{s^{\prime}}=\Sigma \omega^{t \prime} \wedge \omega_{t^{\prime}}^{s^{\prime}}+\Omega^{s^{\prime}}, \\
& \omega_{t}^{s}+\omega_{s^{\prime}}^{t^{\prime}}=0,
\end{aligned}
$$

où les formes $\Omega^{s}$ et $\Omega^{s^{\prime}}$ qui définissent la torsion de la connexion s'écrivent :

$$
\begin{aligned}
& \mathbf{Q}^{s}=\Sigma\left(A_{t h}^{s} \omega^{t} \wedge \omega^{h}+A_{t^{\prime} h^{\prime}}^{s} \omega^{t^{\prime}} \wedge \omega^{h^{\prime}}+B_{t h}^{s} \omega^{t} \wedge \bar{\omega}^{h}+B_{t h^{\prime}}^{s} \omega^{t} \wedge \bar{\omega}^{h^{\prime}}+B_{t^{\prime} h}^{\mathbf{s}} \omega^{t^{\prime}} \wedge \overline{\boldsymbol{\omega}}^{h}+\right. \\
& \left.+B_{t h^{\prime} \omega^{t \prime}}^{s} \wedge \bar{\omega}^{h^{\prime}}+C_{t h}^{s} \bar{\omega}^{t} \wedge \bar{\omega}^{h}+C_{t h^{\prime} \omega^{t}}^{s} \wedge \bar{\omega}^{h^{\prime}}+C_{t^{\prime} h^{\prime}}^{s} \bar{\omega}^{t \prime} \wedge \bar{\omega}^{h^{\prime}}\right),
\end{aligned}
$$

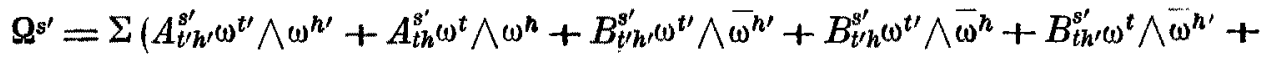

$$
\begin{aligned}
& \text { avec: } \quad A_{t h}^{s}+A_{h t}^{s}=0, \quad A_{t^{\prime} h^{\prime}}^{s}+A_{h^{\prime} t^{\prime}}^{s}=0, \quad C_{t h}^{s}+C_{h t}^{s}=0, \quad C_{t^{\prime} h^{\prime}}^{s}+C_{h^{\prime} t^{\prime}}^{\mathrm{s}}=0, \\
& A_{t^{\prime} h}^{s^{\prime}}+A_{h^{\prime} t^{\prime}}^{g^{\prime}}=0, \quad A_{t h}^{s^{\prime}}+A_{h t}^{s \prime}=0, \quad C_{t^{\prime} h^{\prime}}^{s^{\prime}}+C_{h^{\prime} t^{\prime}}^{s,}=0, \quad C_{t h}^{s^{\prime}}+C_{h t}^{s}=0, \\
& B_{t h}^{s}=B_{s^{\prime} h}^{t^{\prime}}, \quad B_{t h^{\prime}}^{s}=B_{s^{\prime} h}^{t \prime} \text {. }
\end{aligned}
$$

On peut de même définir un tenseur de courbure ainsi qu' un scalaire de courbure, une courbure conforme et une torsion conforme.

Si la structure $\mathfrak{S}$ dérive d'une structure complexe, on peut la désigner par presque parahermitienne complexe; si de plus les deux champs $C_{1}$ et $C_{2}$ sont complètement intégrables, la structure sera dite parahermitienne complexe.

La recherche de celles des structures \& qui sont isotropes (cfr. § 23) conduit aux structures $\mathcal{S}$ intégrables et à des structures localement équivalentes à une structure parahermitienne complexe sur la variete $P_{p}(C) \times P_{p}(C)$, $P_{p}(C)$ désignant l'espace projectif complexe à $p$ dimensions. On obtient ainsi un espace riemannien symétrique complexe.

En se limitant à une isotropie restreinte (transformations unimodulaires), on est conduit en outre à des structures localement equivalentes à une structare presque parahermitienne complexe sur la quadrique complexe à 6 dimensions $Q_{6}(C)$. Cette structure sur $Q_{6}(C)$ admet comme groupe d'automorphismes le groupe simple à 14 paramètres dont $G_{2}$ et $G_{2}^{\prime}$ sont les formes réelles et dont les équations de structure sont les équations (23.7) et (23.8), où l'on suppose les formes $\omega^{s}$, $\omega^{s^{\prime}}$ complexes et linéairement indépendantes dans le domaine complexe.

La structure peut être déterminée par la donnée de deux champs de génératrices supplémentaires de dimension 3 , isomorphes à $P_{\mathrm{s}}(C)$ [24]. 


\section{BIBLIOGRAPHIE}

[1] U. AMALDr, Teoria dei gruppi continui infiniti di trasformazioni, Roma, 1942.

[2] S. Bochner, Curvature in Hermitian metric, "Bull. Amer. Soc. ", 53, 1947, p. 179-195.

[3] - - Curvature and Betti Numbers, Ann. Math. ", 49, 1948, p 379-390.

[4] A. Borel, \& Bull. Amer. Soc. *, 55, 1948, p. 586.

[5] Bourbakr, Algèbre, chap. II, Paris, Hermann, 1947.

[6] - - Algebre, chap. III, Paris, Hermann, 1948.

[7] L. Calabr, Sur les extensions des groupes topologiques, Thèse, Strasbourg, 1951 et - Annali di Matematica, 1951.

[8] E. Cartan, Thèse, Paris, Vuibert, 1982, (2 édition).

[9] - - Sur la structure des groupes infinis de transformations, "Ann. Sci. Ec. Norm. ", 21, 1004, p. 153-206.

[10] - - Les sous-groupes des groupes continus de transformations, ann. Sci. Ec. Norm. s, 25,1908 , p. $57-194$.

[11] - - Les groupes réels simples finis et continus, "Ann. Soj. Ec. Norm. *, 31, 1914, p. 263-355.

[12] - - La structure des groupes infinis, . Séminaire de Math. ", $4^{\ominus}$ année, 1936-37, G, (polycopie).

[13] - - Les problèmes d'équivalence, Selectas, Paris, Gauthier-Villars, 1987, p. 113-136.

[14] - - Les systèmes differentiels extérieurs, Paris, Hermann, 1945.

[15] - Leçons sur la géométrie des espaces de Riemann, Paris, Gauthier-Villars, 1946, ( $2^{e}$ édition).

[16] H. Cartan, Systèmes de Pfaff, , Séminaire de Math. *, $4^{\ominus}$ année 1986-37, B et C.

[17] S. Chern, Some niew viempoints in Differential Geometry in the Large, "Bull. Amer. Soc. $»$, 52. 1946, p. 1-130.

[18] - Characteristic classes of Hermitian manifolds, *Ann. of Math. *, 47, 1946, p. 85.

[19] - - Topics in Differential Geometry, The Institute for Advanced Study, Princeton, 1951, (polycopie).

[20] C. Chevaluey, Theory of Lie Groups, Princeton Un. Press, 1946.

[21] Dieudonné, Sur les systèmes maximaux d'involutions conjuguées et permutables dans les groupes projectifs, Summa Brasiliensis Math., 1950.

[22] B. ECKMANM - H. GUGGENHEIMfr, Formes differentielles et métrique hermitienne sans torsion, "C. R. Acad. Sc. Paris », 229, 1949, p. 464, p. 489.

[28] C. Ehresmann, Sur les espaces localement homogènes, Enseign. Math., 1987, p. 31.

[24] - - Sur la théorie des espaces fibrés, Colloq. Top. Alg. CNRS., Paris, 1947 - Sur les variétés presque complexes, Proc. Inter. Cong. Math., 1950, p. 412.

[25] - - Les connexions infinitésimales dans un espace fibré différentiable, Colloq. Top. Bruxelles, 1950, p. 29.

[26] - - Les prolongements d’une variété différentiable, "C.R. Acad. Sc. Paris *, 238, 1951, p. 598, 777 et 1081 - Structures locales et structures infinitésimales, ibidem, 284, 1952, p. 587.

[27] - - Structures locales, Conférence polycopiée, Rome, Mars 1952.

[28] - - Les prolongements d'une variété différentiable, Congr. Un. Matem. It. Taormina, 1951.

[29] C. Ehresmann-P. Libermann, Sur les formes différentielles extérieures de degré 2 . $\mathrm{C}$. R. Acad. Sc. Paris », 227, 1948, p. 420, - Le problème d'équivalence des formes extévieures quadratiques, ibidem, 229, 1949, 697.

[30] - - Sur les structures presque hermitiennes isotropes, "C. R. Acad. Sc. Paris», 232, 1951, p. 1281.

[31] H. Guggenhemer, Variétés symplectiques, «Colloq. Top. Strasbourg ", 1951, (polycopié).

[32] A. Kirchnoff, Sur l'existence de certains champs tensoriels sur les sphères, × C. R. Acad. Sc. Paris », 225, 1947, p. 1258. 
[33] H. C. LEE, A kind of even-dimensional geometry and its applications to exterior calculus, - Amer. Jour. of Math. s, 55, 1943, p. 433.

[34] Th. Lepage, Sur certaines congruences de formes alternées, « Bull. Soc. Roy. Sc. Liège *, 1946, p. 21-31 - G. PAPY, Sur la divisibilité des formes alternées, ibidem, 1847, p. 24.

[35] - - Sur les matrices symétriques et les modules de formes alternées, «Bull. Acad. Roy. Belgique *, 1949, p. 323.

[36] A. Lichnerowicz, Sur les formes harmoniques des variétés riemanniennes localement réductibles, . C. R. Acad. Se. Sc. Paris ,, 232, 1951, p. 1634.

[37] P. Libnrman, Problèmes d'équivalence relatifs à une structure presque complexe * Bull. Acad. Roy. Belgique ; 36, 1950.

[38] - - Sur la courbure et la torsion des variétés presque hermitiennes, « C. R. Acad. Sc. Paris *, 233, 1951, p. 17.

[39] - - Sur les variétés presque complexes $\nabla_{2 n}$ munies d'un champ de n-éléments réels, C. R. Acad. Sc. Paris ", 233, 1951, p. 1571 - Sur les structures presque quaternioniennes de deuxième espèce, ibidem, 234, 1952, p. 1030.

[40] - - Formes différentielles sur une variété symplectique, C C. R. Acad. Sc. Paris 2, 234, 1952, p. 395, - Colloq. Top. Strasbourg *, 1951, (polycopie).

[41] - - Sur les structures presque paracomplexcs, C. R. Acad. Sc. Paris *, 234, 1952, p. 2517 - Colloq. Top. Strasbourg », 1952, (polycopie).

[42] DE Rham-Brdal, Les formes différentielles harmoniques, “Com. Helv. ", 19, 1949, p. 1.

[43] C. Riqurer, Méthode des fonctions majorantes et systemes d'équations aux dérivées pur. tielles . Mémorial des Sc. Math. ", XXXII, 1028.

[44] J. M. Thomas, Differential systems, *Amer. Math. Soc. Colloq. Publ. », XXI, 1937.

[45] - - An existence theorem for generalized Pfaffian Systems, Bull. Amer. Math Soc.", $\mathrm{XL}, 1984$, p. 309.

[46] A. WELL, Sur la théorie des formes différentielles attachées à une variété analytique complexe, "Com, Helv. ", 20, 1947, p. 110.

[47] YEN Chin TA, Sur l'équivalence des formes différentielles extérieures à quatre variables, . C. R. Acad. Sc. Paris ", 227, 1948, p. 817 ot p. 1203. 